\title{
Placental hypoxia
}

Citation for published version (APA):

Vangrieken, P. (2020). Placental hypoxia: vascular and mitochondrial toxicity. [Doctoral Thesis, Maastricht University]. Maastricht University. https://doi.org/10.26481/dis.20200709pg

Document status and date:

Published: 01/01/2020

DOI:

10.26481/dis.20200709pg

Document Version:

Publisher's PDF, also known as Version of record

\section{Please check the document version of this publication:}

- A submitted manuscript is the version of the article upon submission and before peer-review. There can be important differences between the submitted version and the official published version of record.

People interested in the research are advised to contact the author for the final version of the publication, or visit the DOI to the publisher's website.

- The final author version and the galley proof are versions of the publication after peer review.

- The final published version features the final layout of the paper including the volume, issue and page numbers.

Link to publication

\footnotetext{
General rights rights.

- You may freely distribute the URL identifying the publication in the public portal. please follow below link for the End User Agreement:

www.umlib.nl/taverne-license

Take down policy

If you believe that this document breaches copyright please contact us at:

repository@maastrichtuniversity.nl

providing details and we will investigate your claim.
}

Copyright and moral rights for the publications made accessible in the public portal are retained by the authors and/or other copyright owners and it is a condition of accessing publications that users recognise and abide by the legal requirements associated with these

- Users may download and print one copy of any publication from the public portal for the purpose of private study or research.

- You may not further distribute the material or use it for any profit-making activity or commercial gain

If the publication is distributed under the terms of Article $25 \mathrm{fa}$ of the Dutch Copyright Act, indicated by the "Taverne" license above, 


\section{Placental hypoxia: vascular and mitochondrial toxicity}

Philippe Vangrieken 


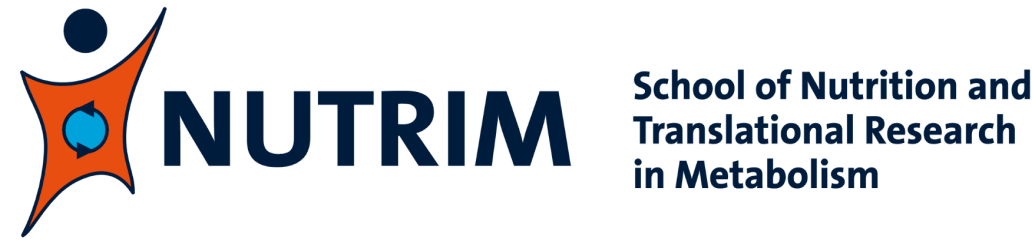

The studies presented in this thesis were performed at the NUTRIM School of Nutrition and Translational Research in Metabolism. These studies were supported by a grant from the NUTRIM Graduate Programme.

Cover design: Philippe Vangrieken

Cover image: Placenta Philine Vangrieken and hand of Philippe and Philine Vangrieken

Layout: Philippe Vangrieken

Printed by: Begas Drukkerij || www.drukkerijbegas.be

ISBN: $\quad$ 978-94-6380-855-2

(C) Copyright Philippe Vangrieken, Maastricht 2020. 


\title{
Placental hypoxia: vascular and mitochondrial toxicity
}

\author{
Proefschrift \\ Ter verkrijging van de graad van doctor aan de Universiteit Maastricht, \\ op het gezag van de Rector Magnificus, Prof. dr. Rianne M. Letschert, \\ volgens het besluit van het College van Decanen, \\ in het openbaar te verdedigen op \\ donderdag 9 juli 2020 om 10:00 uur.
}

door

\section{Philippe Vangrieken}

Geboren te Lommel op 29 april 1992, België 


\section{Promotoren}

Prof. dr. A. Bast

Prof. dr. F.J. van Schooten

\section{Copromotoren}

Dr. S. Al-Nasiry

Dr. P.M.H. Schiffers

\section{Beoordelingscommissie}

Prof. dr. E. Köhler (Voorzitter)

Dr. R.W.L. Godschalk

Dr. S.J. Gordijn (University Medical Center Groningen)

Prof. dr. F.G.M. Russel (Radboud University Medical Center)

Prof. dr. H.A.J. Struijker-Boudier 


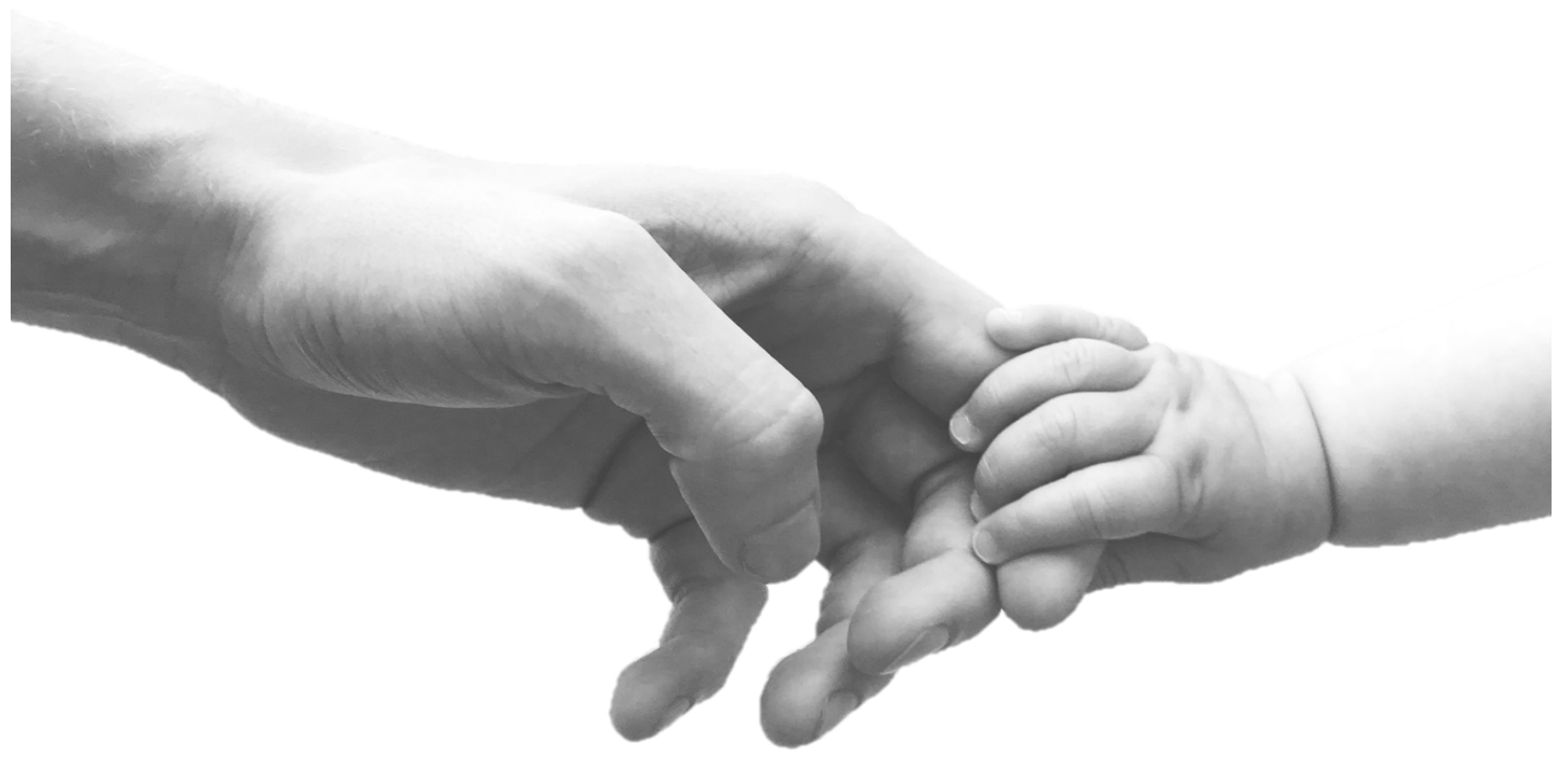

Dit boek draag ik op aan mijn lieve dochter Philine Vangrieken. 


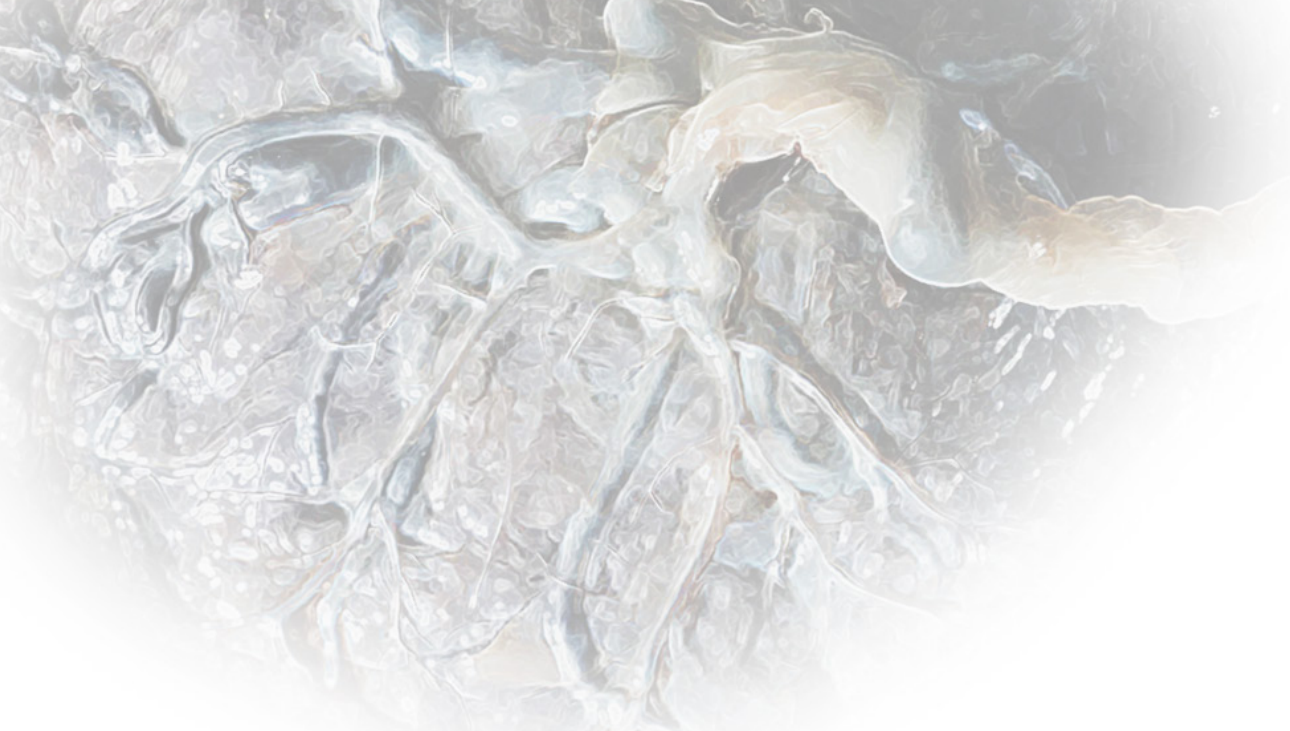




\section{Table of contents}

$\begin{array}{lll}\text { Chapter } 1 \text { General introduction } & 9\end{array}$

Chapter 2 Histological villous maturation in placentae of complicated 29 pregnancies

Chapter 3 Placental mitochondrial abnormalities in preeclampsia 55

Chapter 4 Placental mitochondrial abnormalities upon hypoxia; the 89 cause of placental oxidative stress?

Chapter 5 The direct and sustained consequences of severe placental hypoxia on vascular contractility in preeclampsia

Chapter 6 Consequences of placental hypoxia on vascular contractility, 145 morphology and endothelial integrity

Chapter 7 General discussion and summary

Nederlandse samenvatting

Valorisation

Dankwoord

Publications

Curriculum Vitae 


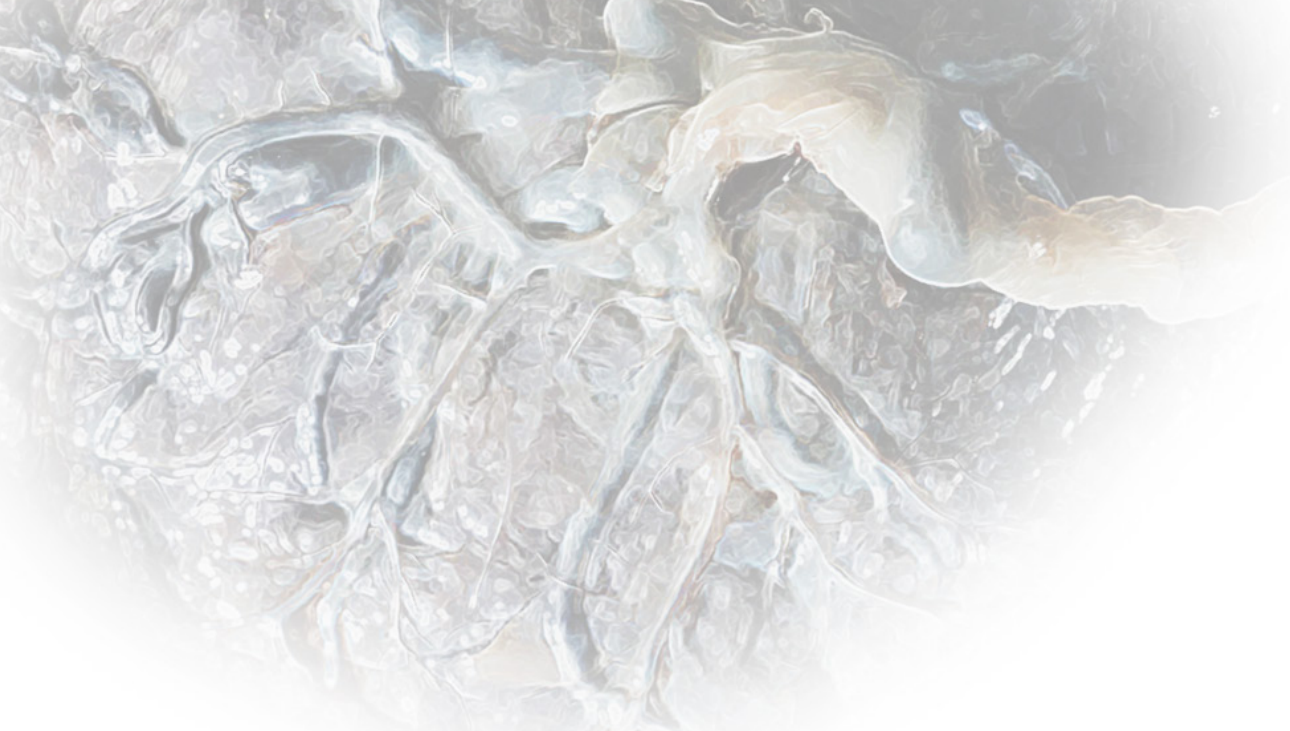




\section{Chapter 1}

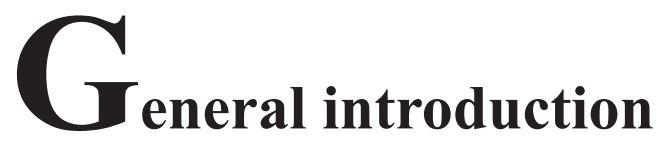




\section{Background}

Preeclampsia (PE) is an enigmatic and complex disorder affecting seemingly healthy pregnant women, commonly in their first pregnancies and carries substantial health risks to both mother and baby. PE is characterized by generalized maternal endothelial dysfunction during the second half of pregnancy, diagnosed by the development of hypertension after the 20th week of gestation. Despite significant research, PE continues to affect 10 million pregnant women and kills 76,000 mothers and 500,000 babies per year worldwide (1). The broad clinical picture of this complication causes a delayed diagnosis, increasing the risk for complications for mother and child. PE is associated with impaired perfusion of the placenta, predisposing the placenta to hypoxia-reoxygenation, which is a potent inducer for placental oxidative stress and many of the changes observed in PE (2-4). PE causes short and long term consequences related to future metabolic and cardiovascular events for the mother and child (5). A delay in diagnosis and access to appropriate care is a core cause of the PE-related severe morbidity and mortality worldwide and is the main reason for the lack of curative options besides preterm delivery of the fetus. The ability to predict, prevent, and cure PE is hindered by the lack of understanding of the molecular mechanisms involved in the early causes of the disease and its unknown clinical subtypes. This thesis details the search for reliable histological and molecular parameters associating PE and placental hypoxia with mitochondrial dysfunction and oxidative stress within the placenta and its subsequent vascular consequences. Our findings may serve as a tool in the identification of PE subtypes and developing prediction strategies, early prevention options, and new directions for a possible cure for this devastating disease.

\section{Normal placental development, anatomy and remodeling spiral arteries}

The Placenta (Greek, plakuos= flat cake) is named on the base of its gross anatomical appearance. The development of the placenta begins upon implantation of the blastocyst into the maternal endometrium and is eventually giving rise to placental villi (6). The outer layer of the blastocyst becomes the trophoblast, forming the outer layer of the placenta, which serves as a barrier for the placenta. This trophoblast layer can be divided into two sublayers: the inner cytotrophoblast layer and the outer syncytiotrophoblast. The syncytiotrophoblast layer, a multinucleated continuous cell layer covers the surface of the placenta and is a result of fusion and differentiation of the cytotrophoblast cells, a process also known as trophoblast turn-over (Figure1). The placental villi carry the structure of a branching tree, which originates from anchoring villi that are attaching to the basal plate at the maternal side and stabilize 
the mechanical integrity of the placental-maternal interface. The mother provides oxygenated nutrient-rich blood into the intervillous space via maternal spiral arteries. The exchange of oxygen, nutrients and waste products between the maternal and fetal circulation is mainly facilitated by the terminal villi of the placenta, as they mainly consist out of large coils of fetal vessels $(6,7)$.

\section{Villous trophoblast turnover}

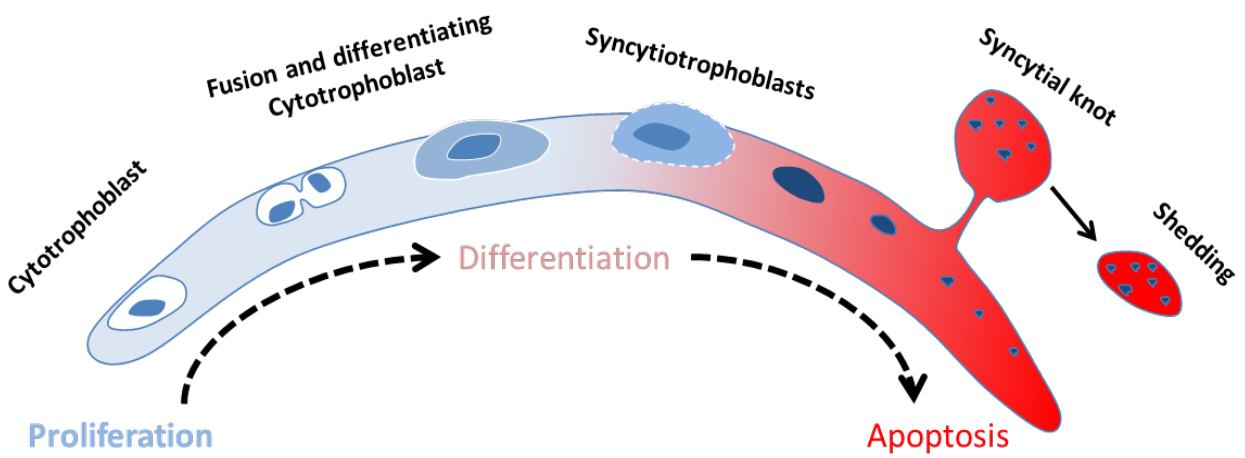

Figure 1. Schematic overview of the villous trophoblast turn-over. Proliferative cytotrophoblast stem cells fuse and differentiate, eventually forming the multinucleated syncytiotrophoblast layer and eventually exit the cell cycle via apoptotic syncytial knots (adapted from Reiter et al (8)).

\section{Placental syndrome}

While most pregnancies progress without complications and result in the delivery of a healthy neonate, a substantial number of pregnancies worldwide (15-25\%) are complicated with one or more disorder of placental development or function, collectively termed "the placental syndrome". The term placental syndrome is gaining popularity above previously used terms such as placental insufficiency and placental bed disorders in modern literature (9). The placental syndrome encompasses a spectrum of pregnancy complications including the two classical phenotypes: Preeclampsia (PE) and fetal growth restrictions (FGR), in addition to gestational hypertension, HELLP syndrome (Hemolysis, Elevated Liver enzymes and Low Platelet count), spontaneous preterm labor, late spontaneous abortion and placental abruption (10). The placental syndrome has been recently associated with disorders of deep placentation starting early in pregnancy with impaired or defective spiral artery remodeling (2, 10-12). PE, the multisystem pregnancy-specific disorder, is considered the prototype of the placental syndrome and is therefore the leading cause of fetal and maternal morbidity and mortality (13). 


\section{Clinical implications of $P E$}

$\mathrm{PE}$ is defined as gestational hypertension $(\geq 140 / 90 \mathrm{mmHg})$ with proteinuria $(\geq 300 \mathrm{mg} / 24 \mathrm{~h}$ ) and there are two sub-types of PE: early- and late-onset PE, with others almost certainly yet to be identified (14). Early-onset PE, occurring before 34 weeks of pregnancy, is typically a more severe form of PE and is widely acknowledged to have a primarily defect in deep placentation, while late-onset PE may center around interactions between senescence of the placenta and a maternal genetic predisposition to cardiovascular and metabolic diseases (14). Numerous predisposing factors have been considered to be related to PE including maternal age, poor nutritional habits, obesity, diabetes and chronic hypertension $(15,16)$. PE is not simply de novo onset of hypertension and proteinuria after 20 weeks of gestation, but rather a syndrome involving multiple organs resulting in end-organ damage in terms of cardiovascular, respiratory, central nervous, renal, and hepatic systems $(17,18)$.

\section{Normal uterine spiral artery remodeling}

As PE presumably originates from early maldevelopment of the placenta, we need to understand how placental vascularization and remodeling takes place, in order to have insight into the pathophysiology of PE. The main uterine arteries give off branches, which extend inward for about a third of the thickness of the myometrium and then subdivide into an arcuate wreath encircling the uterus (19). From this arterial network, smaller radial branches arise in the inner third of the myometrium, better known as uterine spiral arteries (19). During the first trimester of pregnan$\mathrm{cy}$, fetal endovascular trophoblasts originating from the blastocyst-stage invade into maternal spiral arteries as deep as the inner third of the myometrium, accumulate and form plugs in the terminal ends of the maternal spiral arteries. Before 9 weeks of gestation, these trophoblastic plugs only allow blood plasma to seep through when placental perfusion is still minimal $(20,21)$. Before this time, the fetus is engaged in organogenesis and is especially vulnerable to teratogenic damage from free radicals (21). After 9 weeks, the utero-placental arteries recanalize by invasive extra-villous trophoblasts, which initiates spiral artery remodeling by replacing the endothelium and intercalate within the smooth muscle cells of the tunica media $(21,22)$. As illustrated in Figure 2, the resulting 4-fold dilation (up to 2-3 $\mathrm{mm}$ in diameter) of the terminal ends of the spiral arteries transform the arteries into low-resistance vessels and lowers the velocity of the blood flow into the intervillous space by approximately 10 -fold $(2,23)$. These changes allow high maternal blood flow into the intervillous space with a low blood pressure resulting in a continuous perfusion of the intervillous space (19). 


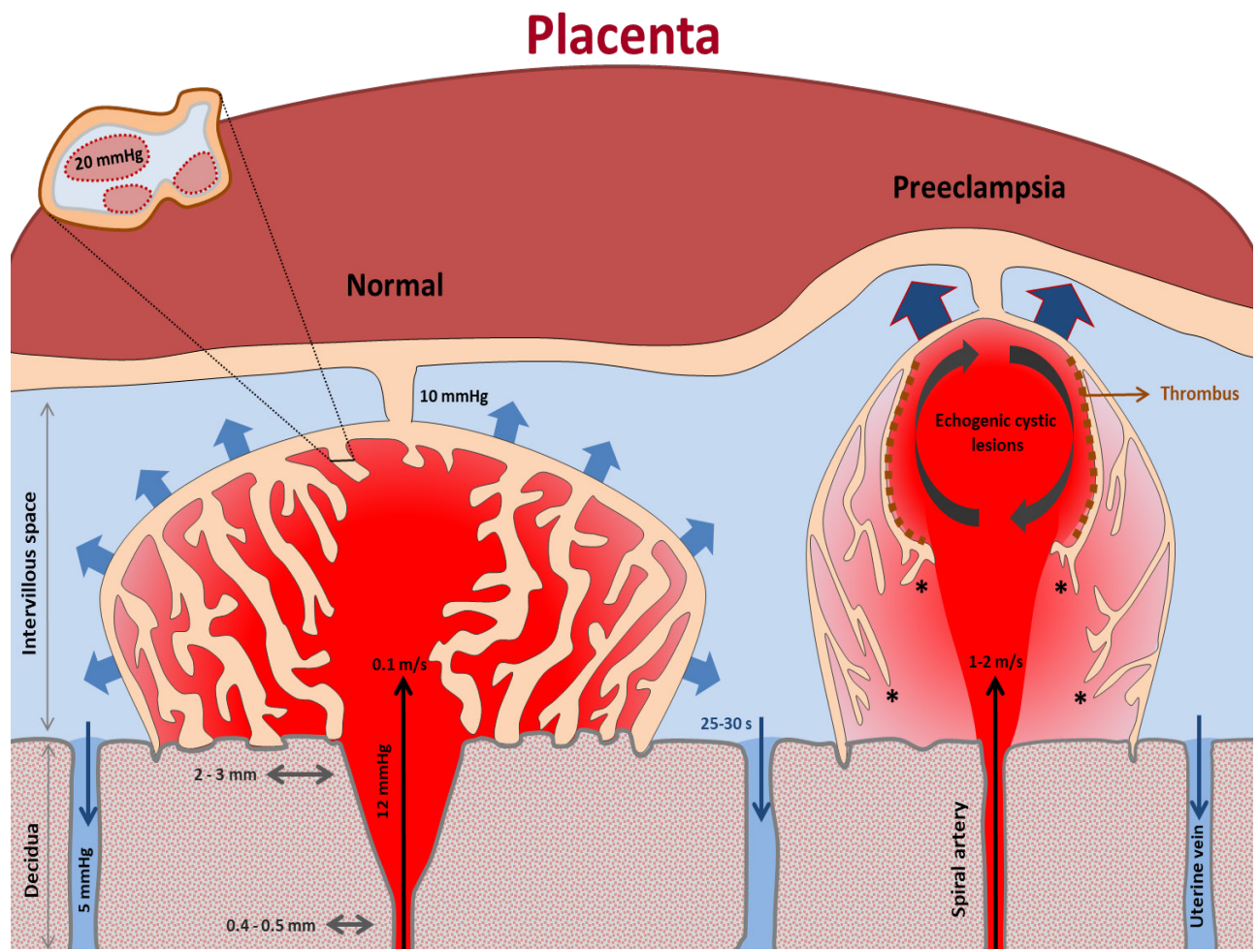

Figure 2. Illustrative representation (not to scale) on the effect of spiral artery remodeling on maternal blood flow into the intervillous space and on lobule architecture predicted by modeling. Dilation of the terminal ends of the spiral arteries in normal pregnancies will reduce the velocity of incoming blood into the central cavity of the placental lobule, from where it will disperse evenly through the villous tree. Transit time from the spiral artery back to the uterine vein is estimated to be in the order of 25-30 s, which allows optimal oxygen exchange. The pressure of the maternal blood is indicated. In the preeclamptic condition, where no or very limited spiral artery remodeling occurs, the maternal blood will enter the intervillous space with a similar pressure, but at a greater velocity. The increased velocity is suggested to rupture the anchoring villi $(*)$ and displaces others to form echogenic cystic lesions lined by thrombus. In the preeclamptic condition, transit time will be reduced resulting in increased oxygen concentrations in the blood leaving the uterine vein (adapted from Burton et al. (2)).

\section{Hypoxia and abnormal uterine spiral artery remodeling in $P E$}

Hypoxia is a condition in which the body or a region of the body is deprived of adequate oxygen supply. This condition can be classified as either generalized hypoxia, where it affects the whole body, or local hypoxia, affecting a region of the body. Although variations in arterial oxygen concentrations can be part of normal physiology, hypoxia is often a pathological condition (24). A considerable portion of the current literature related to PE implicates impaired oxygen supply to the placenta, which compromises placental function and triggers the release of toxic factors 
that subsequently affects maternal and fetal physiology (25). Research on placental hypoxic stress in the PE field is mainly focusing on a defect in spiral arterial remodeling in the first trimester of pregnancy that in turn is caused by perturbation of immunological interactions in the decidua (2). It is well-established that defective trophoblast invasion and failure to convert the spiral arteries are associated with early-onset PE and fetal growth restriction (2, 26-29). Consequently, maternal blood will enter the intervillous space at a greater velocity, which on an ultrasound appears as jet-like streams, supporting the idea of a non-continuous blood flow into the intervillous space. The resulting force is sufficient to drive apart the villous branches, rupture of the anchoring villi and form intervillous lakes, also known as echogenic cystic lesions, which are of prognostic significance by artery Doppler for PE $(2,30)$. The rupture of the anchoring villi will further interrupt invasion of the extra-villous trophoblast and thus also spiral artery modification (Figure 2). As a result of the non-continious blood flow, maternal-fetal oxygen exchange will be impaired, which is in line with the higher oxygen level in the uterine venous blood as reported in cases of fetal growth restriction (31). The resulting intermittent perfusion predisposes the placenta to hypoxia-reoxygenation, which is a potent inducer for placental oxidative stress and many of the changes observed in early-onset PE (2-4). Pregnancy complications related to utero-placental hypoxia have been linked already to the PE phenotype and are known to affect fetal growth $(7,21)$.

\section{Placental maturity in PE}

Since the placenta mediates nutrient and oxygen transport to the fetus, the functionality of its structures plays a fundamental role in fetal growth and development (32). In order to meet the demand of the fast-growing fetus, placental morphology changes as pregnancy progresses. The majority of the fetal portion of the placenta consists of chorionic villous (6). As the placenta matures, the proportion of syncytial knots as a result of the trophoblast turn-over (Figure 1), the number of terminal villi and the diffusion distance between the fetal capillaries and the maternal blood decrease. Indeed, morphologic analysis has revealed that in the early stage of gestation, placental villi are relatively large (approximately $170 \mu \mathrm{m}$ diameter), which progressively decreases in diameter during pregnancy, finally reaching an average diameter of approximately $40 \mu \mathrm{m}$ (19). However, placental maturity may be influenced resulting in a delayed or an accelerated placental maturity. Proper characterization of placental adaptations related to placental maturity, may serve as a diagnostic tool to identify more subtypes of PE for the development of a better/earlier clinical management and may help in the prevention of recurrence of PE during a next pregnancy (Chapter 2). 


\section{Placental mitochondrial dynamics}

It is well-known that disturbed placental perfusion, as observed in patients suffering from PE, can trigger the production of reactive oxygen species (ROS), consequently resulting in the placental release of cytotoxic factors into the maternal circulation (33-35). Recently, mitochondria, a main intracellular source of ROS, have gained more interest as a key player in the hypoxia-induced oxidative stress in PE. In the placenta, disorders related to impaired perfusion including IUGR and PE have recently been shown to be associated with changes in mitochondrial content (36). Besides changes in mitochondrial content, several human studies showed a significant reduction of adenosine triphosphate (ATP) levels in PE placentae (37-39), indicating impaired functioning of the mitochondrial metabolic pathways including the electron transport chain (ETC). Impaired functioning of the ETC is generally associated with excessive mitochondrial ROS formation $(40,41)$. However, whether mitochondrial dysfunction is present in PE placentae or upon placental hypoxic stress and how this contributes to the development of oxidative stress, and the pathophysiology of this disease remains unclear (Chapter 3 and 4). The mitochondria, coined by Carl Benda in 1898 is a double-membrane-bound organelle and can be found back in most eukaryotic organisms and are commonly between 0.75 and $3 \mu \mathrm{m}$ in area (42). Much evidence supports the conclusion that the mitochondria originate from the (eu) bacterial domain of life and engulfed by endocytosis (43). Today, mitochondria still contain their own DNA (mtDNA), which consists of 37 genes. Not all proteins needed for mitochondrial function are encoded by the mitochondrial genome; most are coded by nuclear DNA, where corresponding genes are imported into the mitochondrion (44). In order to coordinate transcription of both the mtDNA and nuclear DNA, the peroxisome proliferator-activated receptor-gamma coactivator family (PPAR- $\gamma$ ) acts as mitochondrial biogenesis master regulator (45). Mitochondrial morphology is highly dynamic. These changes are mediated through mitochondrial fission and fusion events. The fusion process is critical for the maintenance of mitochondrial function, as interruption of this process results in a loss of inner mitochondrial membrane potential (46). Many of the key regulators involved in fission and fusion events have been identified in yeast screens, and most are conserved in mammals. These key regulators include fission mediators dynamin-related 1 (Drp1), and fission 1 (Fis1), as well as the fusion mediators including mitofusin 1/2 (Mfn1/2), which regulate fusion of the outer mitochondrial membrane and optic atrophy protein 1 (Opa1), which promotes fusion of the inner membrane (46-48). Due to the selective regulation of these processes, mitochondria in one cell can be highly heterogeneous in size, function, morphology and mtDNA copy number (49). It has been previously discussed that fusion is involved in the dilution of damaged DNA or proteins in large mitochondrial networks to enable mitochondrial damage repair (49). To ensure 
overall mitochondrial health, the mitochondrial network is able to direct damaged mtDNA and proteins into a specific mitochondrial area, which can then be separated from the main network via mitochondrial fission and broken down via mitophagy (50). There are three different mechanisms for clearing mitochondrial content. First, there is formation and off-budding of small mitochondrial-derived vesicles, which are subsequently cleared via lysosomal breakdown (49). Secondly, there is a mitochondrial ubiquitin-proteasome system, where specific (damaged) proteins are targeted for destruction without altering mitochondrial function (51). Lastly, there is mitochondrial autophagy (mitophagy), which clears complete or large pieces of mitochondria, and is therefore, an important regulator of mitochondrial quantity (49).

\section{Mitophagy}

As extensively reviewed, two main pathways are involved in the initiation of mitophagy $(45,53-55)$ and although often separately described, a crosstalk between these pathways is described (56) (Figure3). The first pathway is the receptor-mediated mitophagy pathway, which selectively targets specific mitochondria by post-translational activation of outer mitochondrial membrane-bound mitophagy receptor proteins (49). Upon activation of these proteins e.g. BCL2/adenovirus E1B $19 \mathrm{kDa}$ protein-interacting protein 3 (BNIP3), BNIP3L or FUN14 domain-containing protein 1 (FUNDC1), binding of these receptor proteins to an autophagosomal-specific protein of the microtubule-associated protein 1A/1B-light chain 3 (LC3) or $\gamma$-aminobutyric acid receptor-associated protein (GABARAP) family is facilitated (49). Subsequently, autophagosomal engulfment and lysosomal degradation are initiated $(49,55)$. The second pathway is specific for mitochondria with a dysfunctional membrane potential (57) and includes the phosphatase and tensin homologue-induced kinase 1 (PINK1)/E3 ubiquitin-protein ligase parkin (Parkin) pathway. In healthy mitochondria, PINK1 is continuously imported into the inner mitochondrial membrane space where it is broken down by the proteasome (49). When the mitochondrial membrane potential is impaired, the import of PINK1 into the inner mitochondrial membrane space is compromised resulting in an accumulation of PINK1 in the outer mitochondrial membrane. Consequently, there it attracts and activates several mitophagy-related proteins of which Parkin has been best described and optineurin (OPTN), which is recruited independently of Parkin as well (58). Furthermore, activated parkin subsequently ubiquitinates several outer mitochondrial membrane-bound proteins, which serve as a docking station for sequestosome 1 (SQSTM1). SQSTM1, is an autophagy receptor, which like the mitophagy receptor proteins as described in the first receptor-mediated mitophagy pathway, is able to bind a member of the autophagosomal-specific protein family, subsequently initiating autophagosomal engulfment and lysosomal degradation $(45,49,53,54)$ (Figure 3). 


\section{Trophoblast cell}

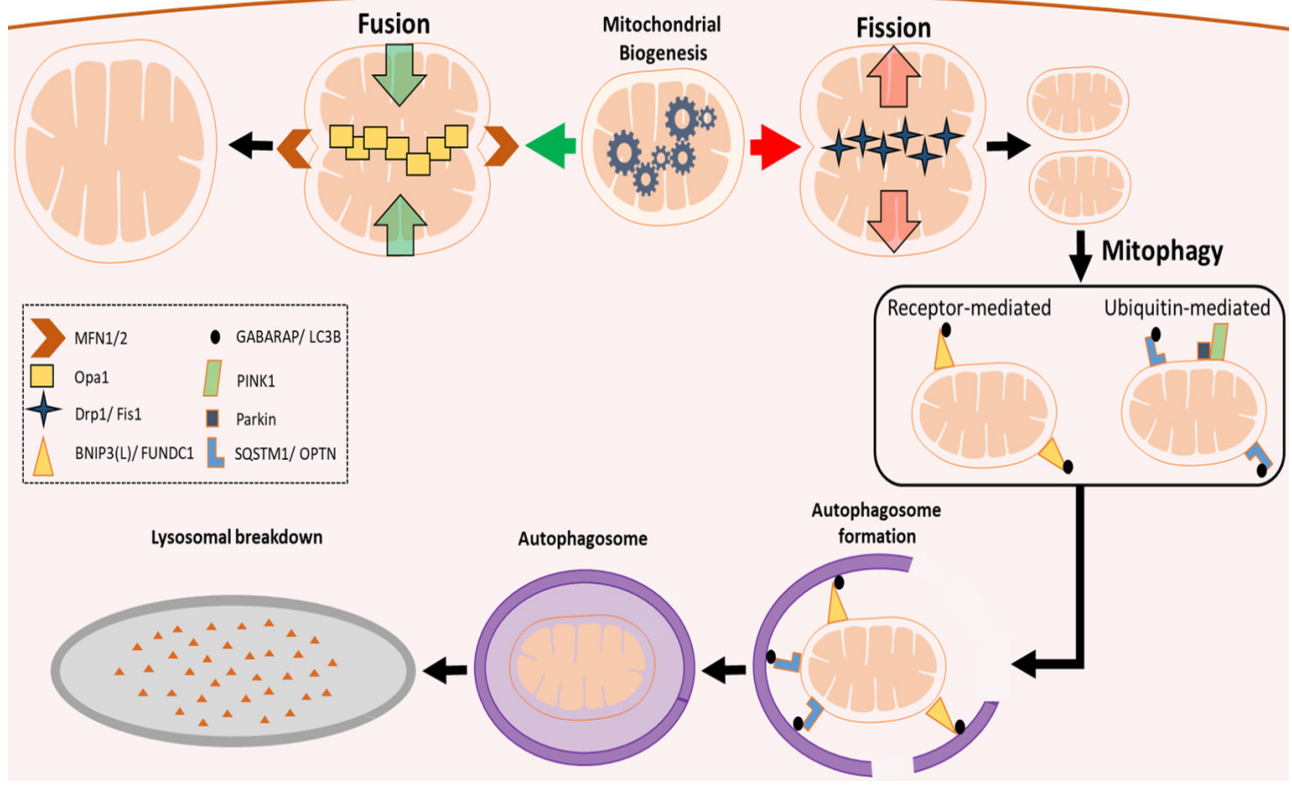

Figure 3. Mitophagy-initiation pathway. Healthy mitochondria will undergo fusion mediated by mitofusin-1/2, which regulates fusion of the outer mitochondrial membrane and optic atrophy protein 1 (Opa1), which promotes fusion of the inner membrane. Damaged mitochondria undergo fission (Fis1)- and dynamin-related protein 1 (Drp1)-mediated fission before mitophagy leaving either parts or complete mitochondria for breakdown. Subsequently, mitochondria are primed for autophagosomal engulfment by GABARAP/LC3B recruitment. GABARAP/LC3B either binds the activated receptor proteins $\mathrm{BNIP} 3 / \mathrm{L}$, or FUNDC1, or in case of a dysfunctional mitochondrial membrane potential to PINK1-recruited Parkin/SQSTM1, or OPTN. Ultimately, GABARAP/LC3B mediates autophagosomal formation around the mitochondrion and subsequent lysosomal breakdown. PINK1: PTEN-induced kinase 1, PARK2: E3 ubiquitin-protein ligase Parkin, FUNDC1: FUN14 domain containing 1, BNIP3: BCL2/adenovirus E1B $19 \mathrm{kDa}$ protein-interacting protein 3, BNIP3L: BCL2/adenovirus E1B $19 \mathrm{kDa}$ protein-interacting protein 3-like, SQSTM1: Sequestosome 1, GABARAPL1: GABA Type A Receptor Associated Protein Like 1, LC3B: Microtubule-associated protein 1 light chain 3 beta I/II, OPTN: Optineurin, LC3A: Microtubule-associated protein 1 light chain 3 alpha, DNM1L: Dynamin-1-like protein, Fis-1: Fission 1 protein, Mfn1: Mitofusin-1, Mfn2: Mitofusin-2 and Opa1: Optic atrophy protein 1 (adapted from Leermakers et al. (49).

Considering the signs for increased oxidative stress and mitochondrial dysfunction found in PE and hypoxia exposed placentae, and the notion that the mitochondria are the main intracellular site for cellular $\mathrm{O}_{2}$ consumption, it is feasible that mitochondria are a significant source for the increased production of placental ROS in PE. Detailed insights into specific mitochondrial abnormalities in $\mathrm{PE}$ and hypoxic placentae and its role in oxidative stress will improve our understanding on the trigger for alterations found in the placental secretome in PE. It may also open new perspectives in a new group directing at mitochondrially targeted therapeutics for PE. 


\section{Maternal vascular consequences of $P E$}

In early-onset PE pathophysiology, impaired uterine spiral artery remodeling is believed to lead to repeated periods of placental hypoxia and reperfusion injury. The resulting oxidative stress is believed to trigger the production of placental factors such as soluble fms-like tyrosine kinase 1 (sFlit1), soluble endoglin (sEng), agonistic auto-antibodies to the angiotensin II type 1 receptor ( $\left.\mathrm{AT}_{1}-\mathrm{AA}\right)$ and inflammatory cytokines (59-62). It has been proposed that these placental secreted factors affect endothelial function and activate the endothelial release of endothelin 1 (ET-1) in the whole maternal circulation, resulting in vasoconstriction and an increase in the contractile responsiveness of the vasculature to vasopressors. In women with a history of PE, these vascular effects have been demonstrated to persist after delivery and are suggested to contribute to an increased risk for cardiovascular diseases in later life (63-65) (Chapter 5 and 6).

ETs are a family of three 21-aminoacid peptides (ET-1, ET-2, and ET-3), of the family, ET-1 is the most prominent member. It is synthesized and secreted by a range of cells, including endothelial cells and the placental syncytiotrophoblast (66). ET secretion occurs constitutively and upon activation of the Weibel-Palade bodies, which are stores of ET in endothelial cells $(66,67)$. Several factors that have been previously linked to PE including angiotensin II, norepinephrine, cytokines, growth factors, hypoxia, ROS but also ET-1 itself, have been reported to induce endothelial ET-1 release $(66,68)$. ET-1 is released towards the basolateral side of endothelial cells, acting primarily as a paracrine or autocrine peptide (69). It is postulated that in severe PE, ET-1 production is so augmented (two- to three-fold) that it loses its paracrine directionality and leads to increased circulating levels and together with $\mathrm{AT}_{1}$-AA induce the local and systemic release of oxidant substances $(66,70-72)$. ETs elicit their effect by binding to the cell-membrane G-protein-coupled ET type A and B (ETA and ETB) receptors, which can be differentiated pharmacologically based on their affinity for the ETs (73). The majority of the ETA receptors are located on vascular smooth muscle cells (VSMC), whereas the ETB receptors are located on endothelial cells, VSMC and epithelial cells $(74,75)$. Activation of ETA and ETB receptors on VSMC induce not only vasoconstriction, but also cell proliferation. The ETB receptor on endothelial cells on the other hand, mediates vascular relaxation by triggering the release of nitric oxide (NO) and prostacyclin $(76,77)$. The ETB receptor is also involved in the clearance of ET-1 by mediating the uptake of ET-1 in kidneys and lungs $(78,79)$. The blockade of ETA receptors like the antagonist Ambrisentan, which is available for clinical use, shows clear potentials for the treatment for PE, but knowing that ET receptor antagonism is teratogenic, underlines the need for other therapeutic options. 
As recently recognized by Burton et al., the synergistic effect of placental factors upon impaired perfusion may explain why understanding the interplay between a hypoxic placenta and vascular abnormalities is so hard, as it is likely that the peripheral aspects of the syndrome are caused by a complex mix of factors rather than any one mediator alone (14). The difficulty to perform mechanistic studies on the complex role of the placenta and the maternal vascular system in pregnant women suffering from PE underlines the need for an in vitro model to test the effect of an altered placental secretome on vascular function and morphology. Next to the placental morphologic adaptations of $\mathrm{PE}$, a better understanding of how a hypoxic placenta results in an altered secretome and its subsequent effects on maternal vascular function and morphology could help in the design of new diagnostic approaches for better prediction and management of PE. Furthermore, it will also pave the way for identifying the long-lasting cardiovascular effects of women after PE and may help in a more directed follow up of these women in later life.

\section{Outline of the thesis}

Aim of part 1 - To assess compensatory accelerated histological villous maturation (AVM) in placentae complicated with PE. Since alterations in the placental secretome in PE is associated with accelerated trophoblast aging of the placenta, we comprehensively asses multiple morphological parameters related to placental hyper maturity. In Chapter 2, preterm placentae complicated by chorioamnionitis or PE were compared to idiopathic preterm placentae and term controls. AVM was analyzed by means of counting numbers of cross-sections of all villi, terminal villi and vasculo-syncytial membranes per villous in CD31-stained sections. Furthermore, the area covered by terminal villi and the proportion of their circumference covered by VSM as well as the area covered by fetal capillaries in terminal villi was measured. In addition, the numbers of syncytial bridges, syncytial apoptotic knots and shed syncytiotrophoblast were counted.

Aim of part 2 - To identify the contribution of mitochondria in mediating placental oxidative stress in PE and hypoxic placentae. In Chapter 3 , in preeclamptic and control placentae, we comprehensively assessed multiple indices of placental antioxidant status, mitochondrial content, mitochondrial biogenesis, mitophagy, and mitochondrial fusion and fission. In addition, we also explored gene expression profiles related to inflammation and apoptosis. $\underline{\text { In Chapter } 4}$, we explored whether abnormalities in mitochondrial metabolism contribute to hypoxia-induced placental oxidative stress by using both healthy term placentae as well as a trophoblast cell line exposed to hypoxia. Furthermore, we explored the therapeutic potential of the antioxidants MitoQ and quercetin in preventing hypoxia-induced placental oxidative stress. 
Aim of part 3 - To determine the effect of a hypoxic placenta on the acute and sustained vascular contractility, morphology, and endothelial integrity. In Chapter 5, Non-complicated human term placentae were collected. Placental explants were subjected to hypoxia and the conditioned media were subsequently added to chorionic arteries mounted into a myograph. Contractile responses of the hypoxic-conditioned media were determined, as well as effects on thromboxane-A2 (U46619) induced contractility. To identify the vasoactive compounds present in the hypoxic-conditioned media, specific receptor antagonists were evaluated. $\underline{\boldsymbol{I n}}$ Chapter 6, Placental villous explants of non-complicated placentae and trophoblasts (BeWo cells) were subjected to hypoxia. The effect of hypoxic-conditioned placental medium on intraluminal-induced contraction and endothelial permeability was investigated using pressure myography. Endothelial cells were exposed to hypoxic-conditioned medium and cell viability, reactive oxygen species formation, and inflammation were determined. Furthermore, morphologic alterations and contractile responsiveness to U46619 in chorionic arteries incubated up to 7 days with hypoxic-conditioned placental medium were examined immunohistochemically and by using wire myography respectively.

Lastly, the results of our studies described in this thesis are discussed in a broader context in Chapter 7. 


\section{References}

1. Giachini FR, Galaviz-Hernandez C, Damiano AE, Viana M, Cadavid A, Asturizaga $\mathrm{P}$, et al. Vascular dysfunction in mother and offspring during preeclampsia: contributions from latin-American countries. Current hypertension reports. 2017;19(10):83.

2. Burton GJ, Woods AW, Jauniaux E, Kingdom JC. Rheological and physiological consequences of conversion of the maternal spiral arteries for uteroplacental blood flow during human pregnancy. Placenta. 2009;30(6):473-82.

3. Hung T-H, Skepper JN, Charnock-Jones DS, Burton GJ. Hypoxia-reoxygenation: a potent inducer of apoptotic changes in the human placenta and possible etiological factor in preeclampsia. Circulation research. 2002;90(12):1274-81.

4. Hung T-H, Charnock-Jones DS, Skepper JN, Burton GJ. Secretion of tumor necrosis factor- $\alpha$ from human placental tissues induced by hypoxia-reoxygenation causes endothelial cell activation in vitro: a potential mediator of the inflammatory response in preeclampsia. The American journal of pathology. 2004;164(3):1049-61. 5. Williams D, editor Long-term complications of preeclampsia. Seminars in nephrology; 2011: Elsevier.

6. Castellucci M kP. Pathology of the human placenta. 5th Edition Ed: Benirschke K, Kaufmann P, Baergen R 2006. 2006:506.

7. Ruiz-Quinonez G, Reza-Lopez SA, Chavez-Corral DV, Sanchez-Ramirez B, Leal-Berumen I, Levario-Carrillo M. Placental maturity, hypertensive disorders of pregnancy and birth weight. Hypertens Pregnancy. 2014;33(2):132-44.

8. Reiter RJ, Rosales-Corral SA, Manchester LC, Tan DX. Peripheral reproductive organ health and melatonin: ready for prime time. Int J Mol Sci. 2013;14(4):723172 .

9. Severens-Rijvers CA, Al-Nasiry S, Vincken A, Haenen G, Winkens B, Ghossein-Doha C, et al. Early-Pregnancy Circulating Antioxidant Capacity and Hemodynamic Adaptation in Recurrent Placental Syndrome: An Exploratory Study. Gynecologic and obstetric investigation. 2019;84(6):616-22.

10. Brosens I, Pijnenborg R, Vercruysse L, Romero R. The "Great Obstetrical Syndromes" are associated with disorders of deep placentation. Am J Obstet Gynecol. 2011;204(3):193-201.

11. Roberts JM. Pathophysiology of ischemic placental disease. Semin Perinatol. 2014;38(3):139-45.

12. Pijnenborg R, Vercruysse L, Hanssens M. The uterine spiral arteries in human pregnancy: facts and controversies. Placenta. 2006;27(9-10):939-58.

13. Scantlebury DC, Hayes SN, Garovic VD. Pre-eclampsia and maternal placental syndromes: an indicator or cause of long-term cardiovascular disease? : BMJ 
Publishing Group Ltd and British Cardiovascular Society; 2012.

14. Burton GJ, Redman CW, Roberts JM, Moffett A. Pre-eclampsia: pathophysiology and clinical implications. BMJ. 2019;366:12381.

15. Pare E, Parry S, McElrath TF, Pucci D, Newton A, Lim KH. Clinical risk factors for preeclampsia in the 21st century. Obstet Gynecol. 2014;124(4):763-70.

16. Sanchez-Aranguren LC, Prada CE, Riano-Medina CE, Lopez M. Endothelial dysfunction and preeclampsia: role of oxidative stress. Front Physiol. 2014;5:372. 17. Vangrieken P, Al-Nasiry S, Janssen GMJ, Weseler AR, Spaanderman ME, Bast A, et al. The direct and sustained consequences of severe placental hypoxia on vascular contractility. PLoS One. 2018;13(8):e0202648.

18. Phipps E, Prasanna D, Brima W, Jim B. Preeclampsia: updates in pathogenesis, definitions, and guidelines. Clinical Journal of the American Society of Nephrology. 2016;11(6):1102-13.

19. Jauniaux E, Jurkovic D, Campbell S. In vivo investigations of the anatomy and the physiology of early human placental circulations. Ultrasound in Obstetrics and Gynecology: The Official Journal of the International Society of Ultrasound in Obstetrics and Gynecology. 1991;1(6):435-45.

20. Weiss G, Sundl M, Glasner A, Huppertz B, Moser G. The trophoblast plug during early pregnancy: a deeper insight. Histochemistry and cell biology. 2016;146(6):749-56.

21. Redman CW, Sargent IL. Latest advances in understanding preeclampsia. Science. 2005;308(5728):1592-4.

22. Soares MJ, Chakraborty D, Kubota K, Renaud SJ, Rumi MA. Adaptive mechanisms controlling uterine spiral artery remodeling during the establishment of pregnancy. Int J Dev Biol. 2014;58(2-4):247-59.

23. Harris JW, Ramsey EM. The morphology of human uteroplacental vasculature: Carnegie Institution of Washington; 1966.

24. Samuel J, Franklin C. Hypoxemia and Hypoxia. In: Myers JA, Millikan KW, Saclarides TJ, editors. Common Surgical Diseases: An Algorithmic Approach to Problem Solving. New York, NY: Springer New York; 2008. p. 391-4.

25. Charnock-Jones DS. Placental hypoxia, endoplasmic reticulum stress and maternal endothelial sensitisation by sFLT1 in pre-eclampsia. Journal of reproductive immunology. 2016;114:81-5.

26. Lyall F, Robson SC, Bulmer JN. Spiral artery remodeling and trophoblast invasion in preeclampsia and fetal growth restriction: relationship to clinical outcome. Hypertension. 2013;62(6):1046-54.

27. Pijnenborg R, Vercruysse L, Hanssens M. The uterine spiral arteries in human pregnancy: facts and controversies. Placenta. 2006;27(9-10):939-58.

28. Brosens JJ, Pijnenborg R, Brosens IA. The myometrial junctional zone spi- 
ral arteries in normal and abnormal pregnancies: a review of the literature. American journal of obstetrics and gynecology. 2002;187(5):1416-23.

29. Gerretsen G, Huisjes HJ, Elema JD. Morphological changes of the spiral arteries in the placental bed in relation to pre-eclampsia and fetal growth retardation. Br J Obstet Gynaecol. 1981;88(9):876-81.

30. Viero S, Chaddha V, Alkazaleh F, Simchen M, Malik A, Kelly E, et al. Prognostic value of placental ultrasound in pregnancies complicated by absent end-diastolic flow velocity in the umbilical arteries. Placenta. 2004;25(8-9):735-41.

31. Pardi G, Cetin I, Marconi AM, Bozzetti P, Buscaglia M, Makowski EL, et al. Venous drainage of the human uterus: Respiratory gas studiesin normal and fetal growth-retarded pregnancies. American journal of obstetrics and gynecology. 1992;166(2):699-706.

32. Jones H, Powell T, Jansson T. Regulation of placental nutrient transport-a review. Placenta. 2007;28(8-9):763-74.

33. Mailloux RJ. Teaching the fundamentals of electron transfer reactions in mitochondria and the production and detection of reactive oxygen species. Redox Biol. 2015;4:381-98.

34. Chiarello DI, Abad C, Rojas D, Toledo F, Vazquez CM, Mate A, et al. Oxidative stress: Normal pregnancy versus preeclampsia. Biochim Biophys Acta Mol Basis Dis. 2018.

35. Wu F, Tian FJ, Lin Y, Xu WM. Oxidative stress: placenta function and dysfunction. American journal of reproductive immunology. 2016;76(4):258-71.

36. Holland O, Dekker Nitert M, Gallo LA, Vejzovic M, Fisher JJ, Perkins AV. Review: Placental mitochondrial function and structure in gestational disorders. Placenta. 2017;54:2-9.

37. Zhou X, Han TL, Chen H, Baker PN, Qi H, Zhang H. Impaired mitochondrial fusion, autophagy, biogenesis and dysregulated lipid metabolism is associated with preeclampsia. Exp Cell Res. 2017;359(1):195-204.

38. Yu J, Guo X, Chen R, Feng L. Downregulation of Mitofusin 2 in Placenta Is Related to Preeclampsia. Biomed Res Int. 2016;2016:6323086.

39. Padmini E, Lavanya S, Uthra V. Preeclamptic placental stress and over expression of mitochondrial HSP70. Clin Chem Lab Med. 2009;47(9):1073-80.

40. Wang Z, Zhang G, Lin M. Mitochondrial tRNA(leu)(UUR) gene mutation and the decreased activity of cytochrome c oxidase in preeclampsia. J Tongji Med Univ. 1999;19(3):209-11.

41. Muralimanoharan S, Maloyan A, Mele J, Guo C, Myatt LG, Myatt L. MIR210 modulates mitochondrial respiration in placenta with preeclampsia. Placenta. 2012;33(10):816-23.

42. Wiemerslage L, Lee D. Quantification of mitochondrial morphology in neu- 
rites of dopaminergic neurons using multiple parameters. Journal of neuroscience methods. 2016;262:56-65.

43. Gray MW, Burger G, Lang BF. The origin and early evolution of mitochondria. Genome Biol. 2001;2(6):REVIEWS1018.

44. Anderson S, Bankier AT, Barrell BG, de Bruijn MH, Coulson AR, Drouin $\mathrm{J}$, et al. Sequence and organization of the human mitochondrial genome. Nature. 1981;290(5806):457-65.

45. Stotland A, Gottlieb RA. Mitochondrial quality control: easy come, easy go. Biochimica et Biophysica Acta (BBA)-Molecular Cell Research. 2015;1853(10):2802-11.

46. Suen DF, Norris KL, Youle RJ. Mitochondrial dynamics and apoptosis. Gene Dev. 2008;22(12):1577-90.

47. Cipolat S, de Brito OM, Dal Zilio B, Scorrano L. OPA1 requires mitofusin 1 to promote mitochondrial fusion. Proceedings of the National Academy of Sciences. 2004;101(45):15927-32.

48. Chen H, Detmer SA, Ewald AJ, Griffin EE, Fraser SE, Chan DC. Mitofusins $\mathrm{Mfn} 1$ and Mfn2 coordinately regulate mitochondrial fusion and are essential for embryonic development. The Journal of cell biology. 2003;160(2):189-200.

49. Leermakers PA, Gosker HR. Skeletal muscle mitophagy in chronic disease: implications for muscle oxidative capacity? Curr Opin Clin Nutr Metab Care. 2016;19(6):427-33.

50. Romanello V, Sandri M. Mitochondrial Quality Control and Muscle Mass Maintenance. Front Physiol. 2015;6:422.

51. Livnat-Levanon N, Glickman MH. Ubiquitin-proteasome system and mitochondria-reciprocity. Biochimica et Biophysica Acta (BBA)-Gene Regulatory Mechanisms. 2011;1809(2):80-7.

52. Anding AL, Baehrecke EH. Cleaning house: selective autophagy of organelles. Developmental cell. 2017;41(1):10-22.

53. Matsuda N. Phospho-ubiquitin: upending the PINK-Parkin-ubiquitin cascade. The Journal of Biochemistry. 2016;159(4):379-85.

54. Wei H, Liu L, Chen Q. Selective removal of mitochondria via mitophagy: distinct pathways for different mitochondrial stresses. Biochimica et Biophysica Acta (BBA)-Molecular Cell Research. 2015;1853(10):2784-90.

55. Liu L, Sakakibara K, Chen Q, Okamoto K. Receptor-mediated mitophagy in yeast and mammalian systems. Cell research. 2014;24(7):787-95.

56. Zimmermann M, Reichert AS. How to get rid of mitochondria: crosstalk and regulation of multiple mitophagy pathways. Biological chemistry. 2017;399(1):2945 .

57. Matsuda N. Phospho-ubiquitin: upending the PINK-Parkin-ubiquitin cas- 
cade. J Biochem. 2016;159(4):379-85.

58. Lazarou M, Sliter DA, Kane LA, Sarraf SA, Wang C, Burman JL, et al. The ubiquitin kinase PINK1 recruits autophagy receptors to induce mitophagy. Nature. 2015;524(7565):309-14.

59. Goulopoulou S, Davidge ST. Molecular mechanisms of maternal vascular dysfunction in preeclampsia. Trends in molecular medicine. 2015;21(2):88-97.

60. Maynard S, Epstein FH, Karumanchi SA. Preeclampsia and angiogenic imbalance. Annu Rev Med. 2008;59:61-78.

61. Naljayan MV, Karumanchi SA. New developments in the pathogenesis of preeclampsia. Adv Chronic Kidney Dis. 2013;20(3):265-70.

62. Seki H. Balance of antiangiogenic and angiogenic factors in the context of the etiology of preeclampsia. Acta Obstet Gynecol Scand. 2014;93(10):959-64.

63. LaMarca B, Amaral LM, Harmon AC, Cornelius DC, Faulkner JL, Cunningham MW, Jr. Placental Ischemia and Resultant Phenotype in Animal Models of Preeclampsia. Curr Hypertens Rep. 2016;18(5):38.

64. Roberts JM, Cooper DW. Pathogenesis and genetics of pre-eclampsia. Lancet. 2001;357(9249):53-6.

65. Henriques AC, Carvalho FH, Feitosa HN, Macena RH, Mota RM, Alencar JC. Endothelial dysfunction after pregnancy-induced hypertension. Int J Gynaecol Obstet. 2014;124(3):230-4.

66. Saleh L, Verdonk K, Visser W, van den Meiracker AH, Danser AJ. The emerging role of endothelin-1 in the pathogenesis of pre-eclampsia. Therapeutic advances in cardiovascular disease. 2016;10(5):282-93.

67. van Mourik JA, de Wit TR, Voorberg J. Biogenesis and exocytosis of Weibel-Palade bodies. Histochemistry and cell biology. 2002;117(2):113-22.

68. Khimji A-k, Rockey DC. Endothelin — biology and disease. Cellular signalling. 2010;22(11):1615-25.

69. Wagner OF, Christ G, Wojta J, Vierhapper H, Parzer S, Nowotny PJ, et al. Polar secretion of endothelin-1 by cultured endothelial cells. J Biol Chem. 1992;267(23):16066-8.

70. George EM, Granger JP. Endothelin: key mediator of hypertension in preeclampsia. Am J Hypertens. 2011;24(9):964-9.

71. Fiore G, Florio P, Micheli L, Nencini C, Rossi M, Cerretani D, et al. Endothelin-1 triggers placental oxidative stress pathways: putative role in preeclampsia. The Journal of Clinical Endocrinology \& Metabolism. 2005;90(7):4205-10.

72. Dong F, Zhang X, Wold LE, Ren Q, Zhang Z, Ren J. Endothelin-1 enhances oxidative stress, cell proliferation and reduces apoptosis in human umbilical vein endothelial cells: role of ETB receptor, NADPH oxidase and caveolin-1. Br J Pharmacol. 2005;145(3):323-33. 
73. Sakurai T, Yanagisawa M, Takuwat Y, Miyazakit H, Kimura S, Goto K, et al. Cloning of a cDNA encoding a non-isopeptide-selective subtype of the endothelin receptor. Nature. 1990;348(6303):732-5.

74. Motte S, McEntee K, Naeije R. Endothelin receptor antagonists. Pharmacology \& therapeutics. 2006;110(3):386-414.

75. Cattaruzza M, Dimigen C, Ehrenreich H, Hecker M. Stretch-induced endothelin B receptor-mediated apoptosis in vascular smooth muscle cells. The FASEB Journal. 2000;14(7):991-8.

76. Lankhorst S, Kappers MH, Van Esch JH, Danser AJ, van den Meiracker AH. Mechanism of hypertension and proteinuria during angiogenesis inhibition: evolving role of endothelin-1. Journal of hypertension. 2013;31(3):444-54.

77. Lavallée M, Takamura M, Parent R, Thorin E. Crosstalk between endothelin and nitric oxide in the control of vascular tone. Heart failure reviews. 2001;6(4):26576.

78. Fukuroda T, Fujikawa T, Ozaki S, Ishikawa K, Yano M, Nishikibe M. Clearance of circulating endothelin-1 by ETB receptors in rats. Biochemical and biophysical research communications. 1994;199(3):1461-5.

79. Dupuis J, Stewart DJ, Cernacek P, Gosselin G. Human pulmonary circulation is an important site for both clearance and production of endothelin-1. Circulation. 1996;94(7):1578-84. 



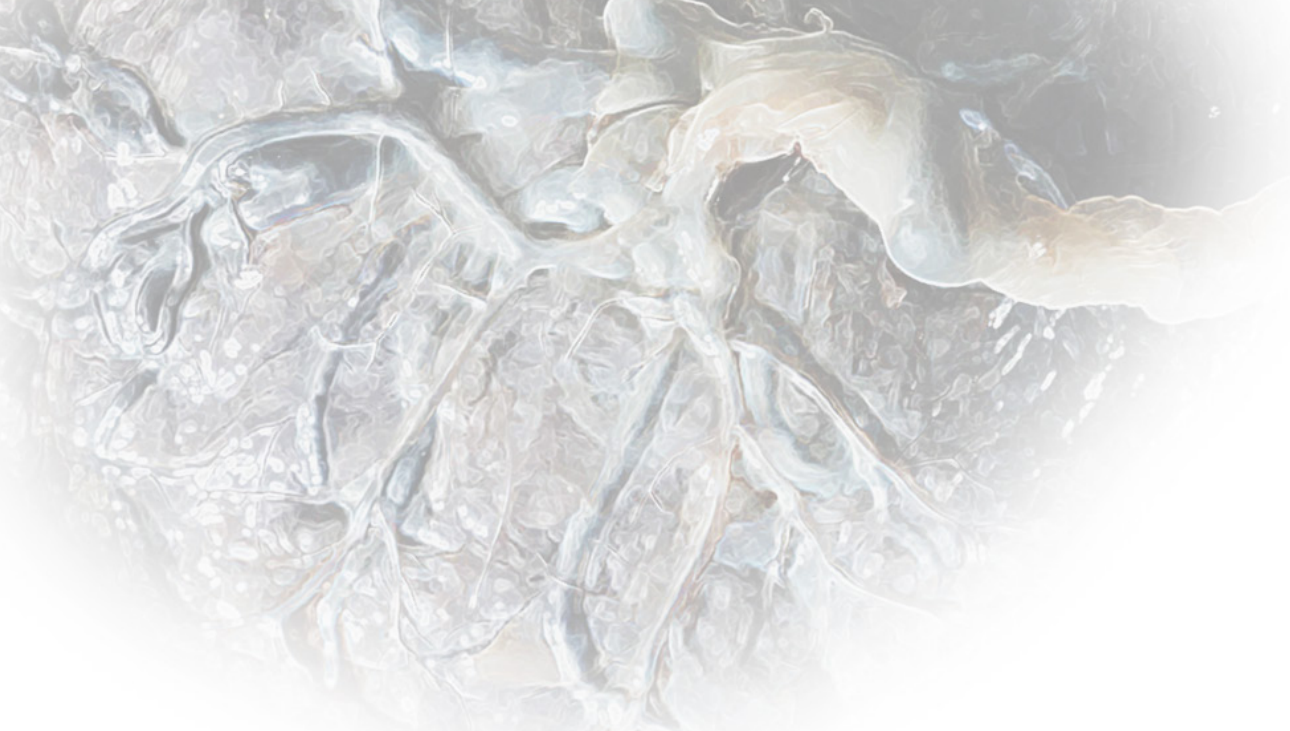




\section{Chapter 2}

\section{$H_{\text {istological villous maturation in placentas }}$ of complicated pregnancies}

Philippe Vangrieken, Sizzle F. Vanterpool, Frederik J. van Schooten, Salwan Al-Nasiry, Peter Andriessen, Ellen Degreef, Joachim Alfer, Boris W. Kramer, Ulrike von Rango

Histol. Histopathol. 2020, 27: 18205. 


\section{Abstract}

Chorioamnionitis and preeclampsia account for the majority of preterm births worldwide. Thus far, adequate methods for early detection or prevention of these diseases are lacking. In preeclampsia, accelerated villous maturation is believed to compensate placental insufficiency. However, little is known about the effects of placental inflammation in chorioamnionitis on villous maturation. Therefore, we established a set of morphological parameters to evaluate histological villous maturity in pregnancies complicated by chorioamnionitis and preeclampsia. Preterm placentas complicated by chorioamnionitis or preeclampsia were compared to idiopathic preterm placentas and term controls. Histological villous maturation was analyzed by means of 17 histological markers. Fourteen of these markers provided information on absolute and relative numbers of the terminal villi (TV), the extent of their vascularization (using CD31-stained sections) and their exchange capacity. In addition, the numbers of syncytial bridges, syncytial apoptotic knots and shed syncytiotrophoblasts were counted. Accelerated villous maturation in preeclampsia was demonstrated by means of histological villous remodeling and confirmed by 11 relevant markers. Chorioamnionitis, however, only showed increased area of fetal capillaries. In preeclampsia, placentas may transition from growth to maturation earlier than placentas in normal pregnancies, whereas in chorioamnionitis placental changes are more acute and therefore less elaborated at a structural level. Regression analysis suggests the number of all villi and the number of terminal villi as a percentage of all villi as parameters to evaluate histological villous maturity in preeclamptic placentas and to assist diagnosis. However, we would recommend to analyze all 11 relevant parameters to judge placental maturity in detail. 


\section{Introduction}

Pregnancy specific pathologies include hypertensive disorders like preeclampsia (PE) and inflammatory related complications such as chorioamnionitis, which affect up to $8 \%$ and $4 \%$ of all pregnancies respectively $(1,2)$. PE is associated with histological abnormalities in the placenta but the exact pathophysiology is still largely unknown $(3,4)$. These pathologies are the primary cause of perinatal mortality worldwide and surviving newborns may face life-long complications (5).

PE and the syndrome of Hemolysis Elevated Liver Enzymes and Low Platelets (HELLP) generally occur after 20 weeks of gestation. The diseases are not simply de novo onset of hypertension and proteinuria, but rather a syndrome involving multiple organs. Their clinical severity ranges from relatively mild to life threatening, being a major cause of severe maternal morbidity and mortality (e.g., stroke, edema and liver rupture) (6-8). This suggests that the disorder has multiple etiologies and probably is multifactorial. The leading hypothesis considers disturbed placental development during the first trimester of pregnancy to be the main cause (6). Impaired remodeling of maternal spiral arteries due to compromised invasion of extra-villous trophoblast cells is thought to precede the development of PE (3, $9,10)$. As a first consequence, reduced trophoblastic plugs may be formed in the lumen of the spiral arteries leading to premature perfusion of the placenta in the first trimester of pregnancy, which may induce oxidative stress in both placental and embryonic cells $(11,12)$. Secondly, the preserved smooth muscle cells in the walls of the spiral arteries and their elastic lamina will reduce dilatation of the terminal ends at the time placental blood flow starts (11). This then leads to increased vascular resistance and reduced local placental perfusion resulting in placental hypoxia and oxidative stress (13). As result of the increased blood pressure at the terminal ends of the spiral arteries, the velocity of the maternal blood reaching the intervillous space will be locally increased and may initiate mechanical damage of the villous trees (11). The products of oxidative stress, such as lipid peroxides are intrinsically pro-inflammatory and initiate increased apoptosis $(11,14)$.

Chorioamnionitis is defined as an acute inflammation of the placental membranes and chorion, which may also include the cord, in which case it is called funisitis (15). The infection is typically due to an ascending poly-microbial bacterial infection in the setting of membrane rupture or very small fastidious genital mycoplasmas such as Ureaplasma species and Mycoplasma hominis when the membranes are still intact (16). Plasmodium vivax and/or probably also P. falciparum can cause pregnancy complications because they are able to induce syncy- 
tial damage and thus can enter the syncytiotrophoblast or even the placental villus $(17,18)$. In cases of clinical chorioamnionitis maternal fever, uterine fundal tenderness, turbid amniotic fluid and maternal/ fetal tachycardia can be found (19). Chorioamnionitis can result in stillbirth, neonatal sepsis, chronic lung diseases, brain injury and maternal postpartum infections and sepsis (20). Symptomatic clinical management is mainly directed to delay preterm birth and limit fetal and maternal morbidity and mortality (6). Histological chorioamnionitis is diagnosed in the placenta after birth by diffuse infiltration of neutrophils in different placental sites. Intra-amniotic infection is generally considered to be the main cause of acute chorioamnionitis and funisitis. Nonetheless, a "sterile" intra-amniotic inflammation can occur in the absence of evidence of colonization by microorganisms (21).

Currently, little is known about histological villous maturation in chorioamniotic placentas. As the recurrence risk of preeclampsia and especially preterm birth with histological chorioamnionitis is high (22), it is mandatory to be able to identify placental aberrations and to make a definitive diagnosis of the underlying pathology.

The morphology of the placenta changes as pregnancy progresses in order to increase the efficiency of the exchange of nutritional compounds and O2/CO2 between mother and child. Placental maturation is generally associated with an increase in diffusion surface and a decrease of the diffusion distance (4). The degree of differentiation of terminal villi (TV) accounts for the placental efficiency as they contain large coils of fetal vessels. Their vascular endothelial basal membrane is partially fused to the basal membrane of the syncytiotrophoblast forming the so-called vasculo-syncytial membranes (VSM). The number and length of these VSM as well as number and circumference of the fetal capillaries are useful parameters to assess placental efficiency (Figure 1) $(3,6,8,11)$.

In addition to the markers mentioned above which focus on the efficiency of placental exchange, syncytial knotting is widely accepted as a reflection of placental maturation and accelerated histological villous maturation (AVM) (23-26). Syncytial knotting has been attributed to pathologically increased compensatory villous branching $(25,27,28)$. It represents the extent of tangential sectioning of syncytiotrophoblast (and therefore probably the extent of villous branching) as well as the number of syncytial apoptotic knots (AK) as the final event of the trophoblast turnover cascade. Therefore, syncytial knotting is an important characteristic of mature placentas (29). AK eventually are shed into the maternal circulation $(25,27,28)$. 

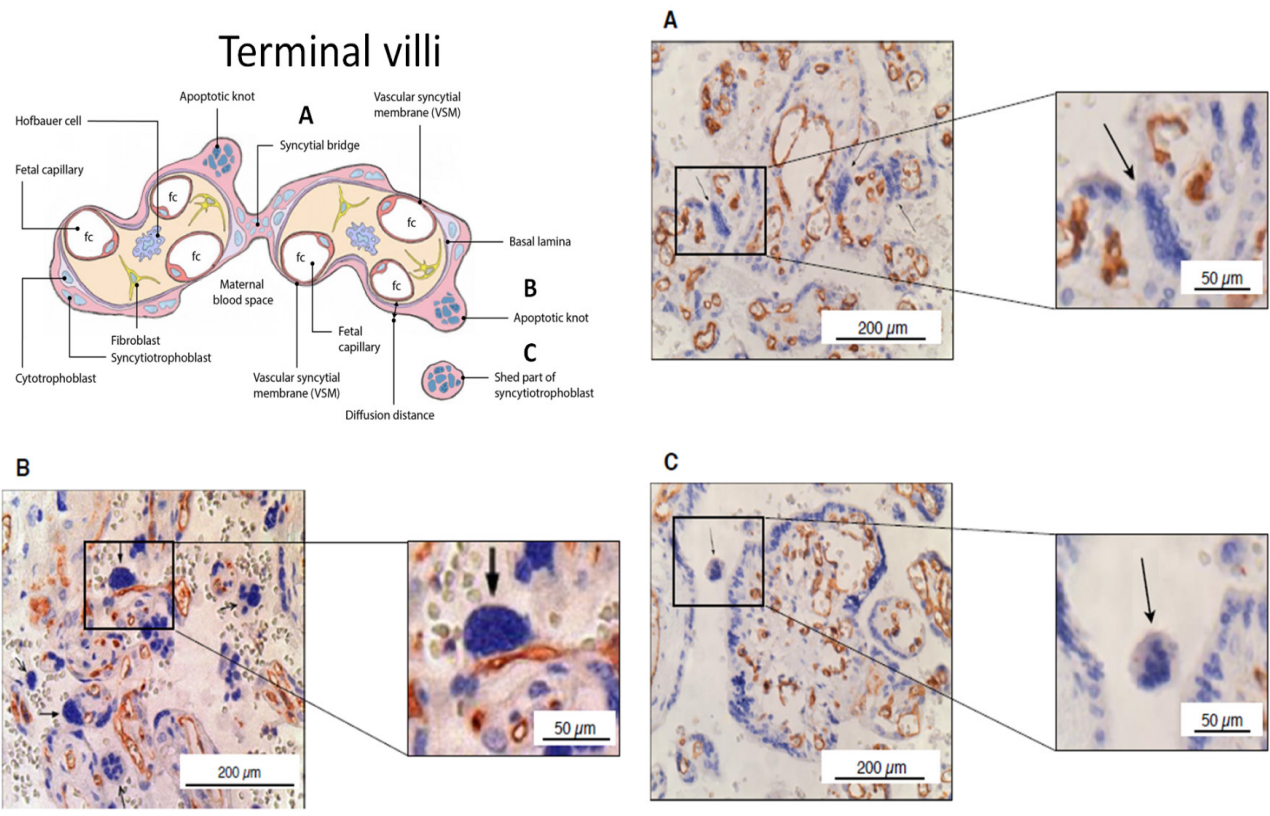

Figure 1. Schematic example of a cross-section of a terminal villi including all investigated parameters. An example of a Syncytial bridge (A), Apoptotic knot (B) and Shed part of syncytiotrophoblast (C), all indicated with arrows.

It is already suggested that adverse pregnancy and fetal outcome are linked to placental senescence (30-33). Changes in histological villous maturation may be a placental adaptation in response to the pathology e.g. maternal vascular mal-perfusion $(26,34)$. Histological maturation in placentas of adverse pregnancy outcomes such as preterm birth after PE or chorioamnionitis have not been analyzed in detail in the context of the underlying pathology. However, detailed analysis of histological villous maturation may reveal the pathophysiology. Knowledge of what happens to the placenta during pregnancy may improve follow up of both the mother and child and reduce recurrence risk.

The present study therefore aims to increase the knowledge of specific placental aberrations/adaptations by evaluating a set of parameters of TV maturation that can be used to assess placental maturity and link changes in histological villous maturation to a specific pathology. 


\section{Materials and methods}

\section{Placental specimen}

Preterm placentas from a total of 306 singleton pregnancies were recruited from the public hospital Máxima Medical Center in Veldhoven, The Netherlands between January 1st 2009 and December 31st 2010. PE was diagnosed as new onset hypertension (blood pressure $>140 / 90 \mathrm{mmHg}$ or mean arterial pressure $>105 \mathrm{mmHg}$ recorded on at least two separate occasions) after 20 weeks gestation accompanied by proteinuria (>300 mg/24h) (35). As suggested by the Amniotic Fluid Infection Nosology Committee, placental and umbilical cord samples were assessed for signs of histological chorioamnionitis by determining the presence of polymorphonuclear cells present in the chorionic plate or membranous chorionic connective tissue and/ or the amnion. The diagnosis of histologic chorioamnionitis with funisitis included any of the following features: chorionic vasculitis, umbilical phlebitis, umbilical (pan) vasculitis, (sub-acute) necrotizing funisitis, or concentric umbilical perivasculitis $(1,36)$. Spontaneous idiopathic preterm pregnancies, used as preterm control, were defined as pre-term birth with no known clinical abnormalities. For this study a cohort of 100 preterm placentas from pregnancies complicated by chorioamnionitis (+/- funisitis) (29 \pm 2 weeks), early onset PE (+/- HELLP) (30 \pm 2 weeks) and spontaneous idiopathic preterm pregnancies $(30 \pm 2$ weeks $)$ could be included. Exclusion criteria were: missing of relevant clinical data, multiples, presence of other pathologies, insufficient amount of suitable tissue or poor morphological quality. For accurate morphometric analysis of $\mathrm{TV}$, the assessment of the quality of the samples used for detailed morphometric analysis was stricter in comparison with the assessment of syncytial knotting. Therefore, only 70 samples were suitable for detailed histological analysis of TV. In addition to the preterm cohort, a group of 15 singleton term placentas (39 \pm 1.2 weeks) from singleton pregnancies was collected at Maastricht University Medical Center, 2015-2016 as a positive control for normal complete maturation (see table 1 for clinical data). Placentas of both cohorts were collected immediately after each delivery and fixed for 24-48 hours. The placenta was cut in slices of 1-2 cm to determine the presence of macroscopic lesions. Three blocks of normal placental parenchyma (sampled from the central area of the placenta) were embedded in paraffin, processed and stained with hematoxylin and eosin (1). Blocks showing the best tissue morphology were used for further analysis. 
Table 1. Characteristics of the patients

\begin{tabular}{|c|c|c|c|c|c|c|c|}
\hline Variable & $\begin{array}{c}\text { Idiopathic } \\
\text { preterm control } \\
\text { Mean } \pm \text { SD }\end{array}$ & $\begin{array}{c}\text { Chorioamnionitis } \\
\text { +/- funisitis } \\
\text { Mean } \pm \text { SD }\end{array}$ & $\begin{array}{c}\text { PE } \\
+/- \text { HELLP } \\
\text { Mean } \pm \text { SD }\end{array}$ & $\begin{array}{c}\text { Non-complicated } \\
\text { term control } \\
\text { Mean } \pm \text { SD }\end{array}$ & ND & $p^{1}$ & $\mathbf{p}^{2}$ \\
\hline Number placentas & 24 & 41 & 35 & 15 & I & I & 1 \\
\hline Maternal age (years) & $30 \pm 5$ & $30 \pm 5$ & $32 \pm 4$ & $31 \pm 5$ & Yes & 0.0638 & 0.1209 \\
\hline Gestational age (weeks) & $30 \pm 2$ & $29 \pm 2$ & $30 \pm 2$ & $39 \pm 1$ & No & 0.0752 & $<0.0001$ \\
\hline Birth weight (g) & $1301 \pm 379$ & $1292 \pm 316$ & $1143 \pm 261$ & $3258 \pm 380$ & Yes & 0.0696 & $<0.0001$ \\
\hline Gravidity & $2 \pm 2$ & $2 \pm 2$ & $2 \pm 1$ & $2 \pm 2$ & No & 0.1802 & 0.1182 \\
\hline Parity & $1 \pm 1$ & $1 \pm 2$ & $0 \pm 1$ & $1 \pm 1$ & No & 0.1244 & 0.1043 \\
\hline Fetal gender (male/female), \% & $29 / 71$ & $46 / 54$ & $63 / 37$ & $47 / 53$ & I & I & l \\
\hline SGA, \% & 13 & 4 & 11 & 0 & 1 & l & I \\
\hline C-section, \% & 42 & 17 & 97 & 33 & 1 & I & l \\
\hline
\end{tabular}

PE: Preeclampsia, HELLP: Hemolysis, Elevated Liver enzymes and Low Platelets, ND: Normal distributed, 1p-value including the idiopathic preterm control, the chorioamnionitis (+/- funisitis) and the PE (+/- HELLP) groups, $2 p$-value including all groups, SGA: Small for gestational age.

\section{Ethical aspects}

The collection of the placentas and the use of the placental specimens were done according to Dutch law. The use of placentas from patients of Máxima Medical Center, Veldhoven, was approved by the local Medical Ethical Committee of the Máxima Medical Center, considering that retrospective and anonymous data collection was performed (reference number 20.12.2011). The collection of the 15 term placentas were approved by the Medical Ethics Committee Academic Hospital Maastricht and Maastricht University (METC 16-4-047).

\section{Analysis of the placental weight adjusted for gestational age}

To examine whether preterm placentas show differences in weight between the three preterm groups and to validate our idiopathic preterm control group. Data reported by Kaufmann P, extrapolated from Boyd \& Hamilton and O'Rahilly on the correlation between placental weight and gestational age was used to correlate placental weight in our cohort to the expected placental weight $(37,38)$.

\section{Immunohistochemistry}

To visualize blood vessels, sections were stained for CD-31 PECAM1 (platelet and endothelial cell adhesion molecule 1). Placental sections were first deparaffinized with absolute Xylene and hydrated via graded ethanol series (100\%-50\%-DMEMPBS (Dulbecco's Modified Eagle's Medium-Phosphate Buffered Saline)). Sections were boiled in a sodium citrate solution $(10 \mathrm{mM}, \mathrm{pH}$ 6.0) for 5 minutes and washed twice in DMEM and PBS to unmask relevant epitopes. Endogenous peroxidase was blocked in $0.3 \% \mathrm{H} 2 \mathrm{O} 2 /$ Methanol for 30 minutes and 5\% normal goat serum (NGS) was used to minimize nonspecific antibody binding. After the blocking procedure, 
placental sections were incubated with CD31 PECAM1 (DAKO clone JC70A, Dako Denmark, 1:200 in PBST (Phosphate Buffered Saline with Tween 20)/5\% NGS) overnight at $4{ }^{\circ} \mathrm{C}$. After washing, a secondary antibody: goat anti-mouse (GAM-biotin Vector BA9200; 1:1000 in PBST/5\% NGS) was allowed to bind for 30 minutes at room temperature. HRP coupled Avidin/Streptavidin-biotin complex (ABC (Avidin-biotin-complex) kit Elite, Vectastain PK6100, Vector Laboratories Inc., 30 Ingold Road, Burlingama, CA 94010) was used to amplify the signal and incubated for $30 \mathrm{~min}$ at room temperature. Sections were then incubated with the substrate 3,3'-diaminobenzindine (DAB). Staining was stopped with water and sections were counter-stained using hematoxylin. Finally, placental sections were dehydrated via a graded alcohol series $(70 \%, 90 \%, 96 \%, 2 \times 100 \%)$ and covered with Entellan (Merck KGaA, 64271 Darmstadt, Germany). For negative controls, the first antibody was replaced by buffer.

\section{Analysis of the placental sections}

Sections were examined with a light microscope (Leica DMRXA, Leica Microsystems GmbH, Ernst-Leitz-Strasse 17-37, 35578 Wetzlar, Germany).

Before the analysis, three different areas of each section were systematically randomly sampled. This was done by selecting 3 adequate regions at a 10x magnification including 2 peripheral (left and right corner) and one central region of the placental specimen. Then from each region a random picture was made at a 20x magnification, which was used for our analysis. First, the mean number of all villi and TV cross sections was counted giving a first indication of the degree of villous branching. From these data, the relative amount (\%) of TV were calculated. Based on the definition of Kaufmann et al. 1976, a villus was defined as TV if at least $30 \%$ of its cross sectional surface was covered by fetal capillaries and if it contained at least two VSM (Figure 1) (37). Villous cross sections fulfilling the criteria of a TV and being fully located in the high power field were included in further evaluation routines including area, circumference and distance measurements, using the Leica-QWin standard software. For each TV cross section, the number of fetal capillaries was counted and their cross sectional area was measured. This allows the calculation of the area of the high power field covered by TV and percentage of area occupied by fetal capillaries. In addition, per TV, the number of VSM was counted and their length was measured. Using these data and the measurement of the circumference of the TV cross section allowed us to calculate the percentage of the TV circumference that was covered by VSM (Figure 1, Table 2). The mean diffusion distance per TV represents the average of the shortest distance between the fetal capillaries and the TV membrane (Figure $1)$. 
Table 2. Morphometric analysis of the terminal villi

\begin{tabular}{|c|c|c|c|c|c|c|c|}
\hline Expressed in mean/view & $\begin{array}{c}\text { Idiopathic } \\
\text { preterm } \\
\text { Mean } \pm \text { SD }\end{array}$ & $\begin{array}{c}\text { Chorioamnionitis } \\
\text { +/- funisitis } \\
\text { Mean } \pm \text { SD }\end{array}$ & $\begin{array}{c}\text { PE } \\
+/ \text { - HELLP } \\
\text { Mean } \pm \text { SD }\end{array}$ & $\begin{array}{c}\text { Non-complicated } \\
\qquad \begin{array}{c}\text { Term } \\
\text { Mean } \pm \text { SD }\end{array}\end{array}$ & ND & $\mathbf{p}^{1}$ & $\mathbf{p}^{2}$ \\
\hline Number placentas & 18 & 30 & 22 & 15 & 1 & / & l \\
\hline Number all villi & $35.2 \pm 10.8$ & $31.3 \pm 10.1$ & $44.8 \pm 8.4$ & $38.5 \pm 9.3$ & No & $<0.0001$ & 0.0001 \\
\hline Number TV & $4.7 \pm 4.5$ & $5.3 \pm 4.2$ & $13.5 \pm 8.3$ & $8.9 \pm 5.0$ & No & 0.0001 & $<0.0001$ \\
\hline $\mathrm{TV}, \%$ & $13.9 \pm 12.5$ & $23.5 \pm 17.5$ & $38.6 \pm 23.5$ & $31.9 \pm 18.3$ & No & 0.0018 & 0.0015 \\
\hline Area covered by TV, \% & $3.9 \pm 3.4$ & $7.8 \pm 8.2$ & $13.5 \pm 9.2$ & $14.3 \pm 5.4$ & No & 0.0009 & $<0.0001$ \\
\hline Number FC/ TV & $6.2 \pm 2.8$ & $6.3 \pm 2.0$ & $6.5 \pm 1.6$ & $6.8 \pm 3.3$ & No & 0.2926 & 0.4483 \\
\hline Number VSM/ TV & $2.7 \pm 0.5$ & $3.0 \pm 0.8$ & $3.4 \pm 0.5$ & $3.6 \pm 0.6$ & No & 0.0013 & 0.0001 \\
\hline Total length VSM/ TV ( $\mu \mathrm{m})$ & $42.4 \pm 15.2$ & $47.8 \pm 19.3$ & $54.0 \pm 16.3$ & $71.6 \pm 20.3$ & No & 0.0594 & 0.0004 \\
\hline Length DD/ FC $(\mu \mathrm{m})$ & $3.8 \pm 2.4$ & $4.1 \pm 2.6$ & $2.9 \pm 1.0$ & $3.3 \pm 1.0$ & No & 0.0653 & 0.1314 \\
\hline Circumferences TV $(\mu \mathrm{m})$ & $263.5 \pm 77.4$ & $284.7 \pm 74.1$ & $258.6 \pm 39.6$ & $304.1 \pm 66.1$ & No & 0.2919 & 0.1402 \\
\hline Area $T V\left(\mu \mathrm{m}^{2}\right)$ & $4008.8 \pm 2235.1$ & $5133.7 \pm 2720.3$ & $3768.4 \pm 987.7$ & $4946.2 \pm 1014.0$ & No & 0.1068 & 0.0302 \\
\hline Circumferences FC $(\mu \mathrm{m})$ & $423.8 \pm 215.7$ & $454.1 \pm 168.1$ & $480.1 \pm 326.3$ & $432.8 \pm 78.5$ & No & 0.3225 & 0.4255 \\
\hline Area $\mathbf{F C} / \mathrm{TV}\left(\mu \mathbf{m}^{2}\right)$ & $1400.0 \pm 982.5$ & $2141.7 \pm 1164.2$ & $1652.4 \pm 464.7$ & $2369.3 \pm 1009.6$ & No & 0.0184 & 0.0042 \\
\hline Area $\mathrm{FC} / \mathrm{TV}, \%$ & $35.8 \pm 12.6$ & $41.1 \pm 5.5$ & $43.9 \pm 5.2$ & $45.0 \pm 9.4$ & No & 0.0549 & 0.0677 \\
\hline Circumference VSM/ TV, \% & $16.6 \pm 5.1$ & $17.5 \pm 8.2$ & $21.0 \pm 5.5$ & $24.3 \pm 7.7$ & No & 0.0115 & 0.0017 \\
\hline
\end{tabular}

PE: Preeclampsia, HELLP: Hemolysis, Elevated Liver enzymes and Low Platelet, TV: Terminal villi, FC: Fetal capillary, VSM: Vasculo-syncytial membrane, DD: Diffusion distance, ND: Normal distributed, 1p-value including the idiopathic preterm control, the chorioamnionitis (+/- funisitis) and the PE (+/-HELLP) groups, $2 p$-value including all groups.

In addition to the detailed structure of the TV, syncytial knotting as a maturation characteristic of the syncytiotrophoblast was analyzed. Syncytial knots were defined as aggregates of syncytial nuclei at the surface of terminal villi $(25,39)$. Syncytial bridges appear as inter-villous bridges and tangential flat sectioning according to Jones et al. (Figure 1A) (40). True AK were defined as isolated round or elliptic structures containing at least 10 strong accumulated syncytiotrophoblast pyknotic nuclei projected from the cross sectional villous surface and showing densely packed chromatin according to Johyansen et al. (Figure 1B) (41). If the syncytial/apoptotic knot could not be clearly assigned to a villus (at least one fetal capillary has to be present in the villus) it was classified as a shed syncytiotrophoblast according to Askelund et al. (Figure 1C) (42). Syncytial knots, syncytial bridges, AK and shed parts of the syncytiotrophoblast all together represent syncytial knotting (see Figure 1 and 2 for examples). 

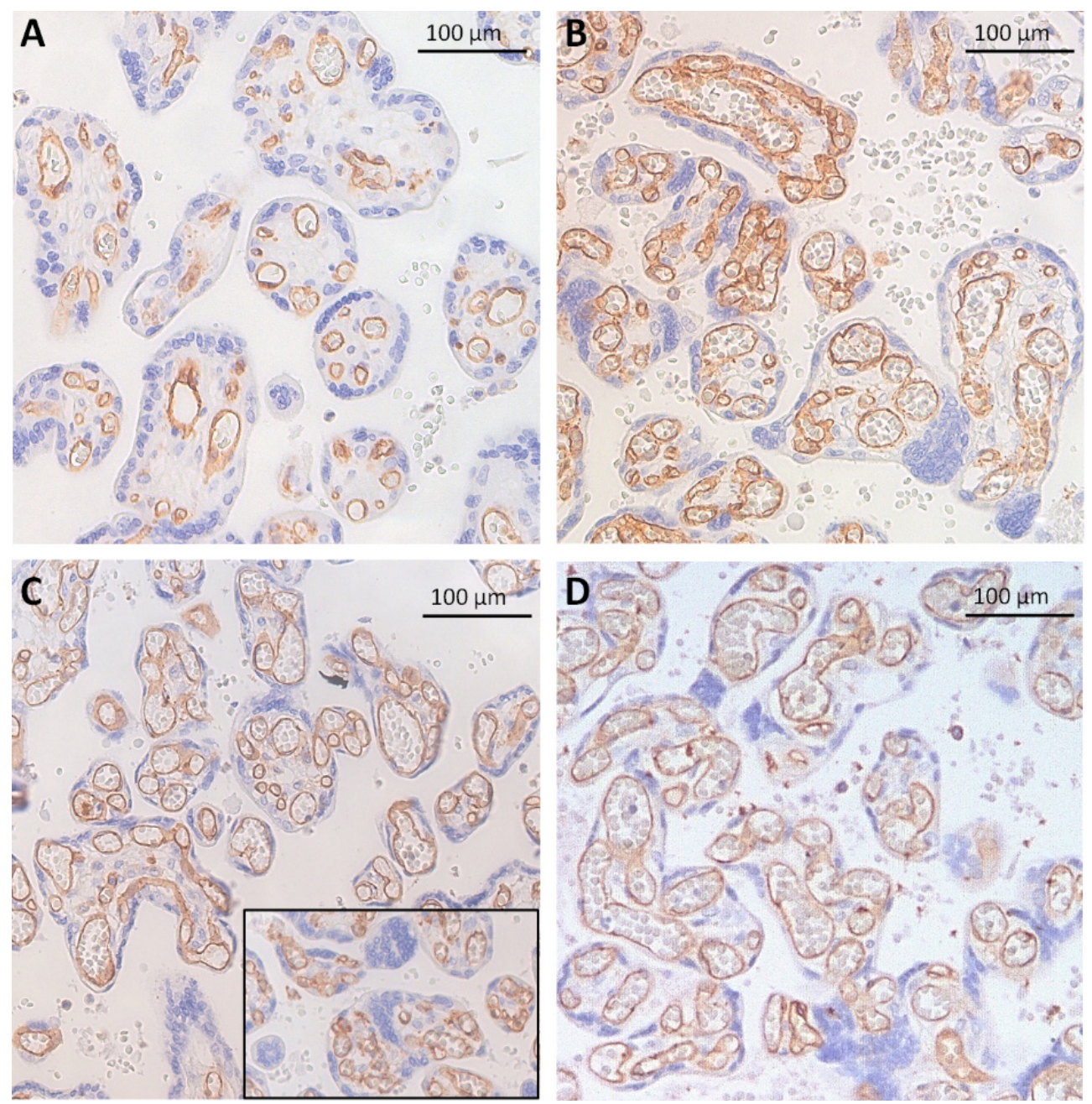

Figure 2. Representative part of the high power field of each group. Idiopathic preterm control (A), Chorioamnionitis (+/- funisitis (B), Preeclampsia (+/- HELLP) (C) and Non-complicated term control (D).

Subclasses of syncytial knotting were characterized by two independent blinded investigators to minimize bias. Values of the Intraclass Correlation Coefficient calculated to evaluate inter-observer agreement were: 0.92 for the shed parts of the syncytiotrophoblast, 0.87 for the AK and 0.78 for the syncytial bridging. Landis and Koch classify scale values of 0 as "no agreement", $0-0.20$ as "slight agreement", 0.21-0.40 as "fair agreement", 0.41-0.60 as "moderate agreement", 0.61-0.80 as "substantial agreement" and 0.81-1 as "perfect agreement" (43). In this rating scheme, the inter-observer variability of the present study is at the edge of "substantial agreement" to "perfect agreement". 
In addition to the TV analysis, immature intermediate villi were counted as well (Figure 3).

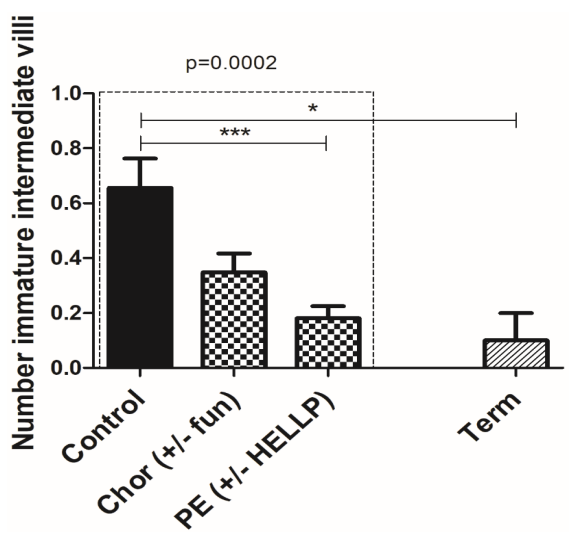

Figure 3. Mean number of immature intermediate villi, fully located in the high power. Data are presented as median with SEM. Ns: $p>0.05,{ }^{*} \mathrm{p} \leq 0.05,{ }^{*} \mathrm{p} \leq 0.01,{ }^{* * *} \mathrm{p} \leq 0.001$. P-values given include all 3 groups.

\section{Statistical analyses}

When the D'agrostino and Pearson omnibus normality test did not find a departure from a Gaussian distribution, the ANOVA one-way analysis of the variance was used followed by the multiple comparison Tukey post-hoc test. If not, the Kruskal-Wallis test was used followed by the Dunn's multiple comparison post-hoc test using GraphPad Prism 6 for Windows, GraphPad Software, La Jolla California USA. A p-value $<0.05$ was considered as significantly different and was presented as follows: Ns: $p>0.05, * p \leq 0.05, * * p \leq 0.01, * * * p \leq 0.001$. Inter-observer agreement was tested by the Intraclass Correlation Coefficient test. Correlates of PE and chorioamnionitis, including all markers for AVM and the clinical parameters used in our study were subjected to multivariate analysis. These correlates were weighted by means of binary stepwise forward logistic regression against each other and only those that demonstrated independence from each other were retained in the model using SPSS (IBM Corp. Released 2016. IBM SPSS Statistics for Windows, Version 24.0. Armonk, NY: IBM Corp). 


\section{Results}

\section{Clinical characteristics of the patients}

Basic parameters of the 4 test groups: the idiopathic preterm group (preterm control), the chorioamnionitis group, the PE group and the non-pathological term group (term control, gestational age $>37$ weeks) were tested for several clinical parameters. All data are presented as means and standard deviation (Table 1). There were no significant differences in clinical characteristics found between all the preterm groups. In the term group gestational age, placental and birth weight were increased as expected (Table 1). Being small for gestational age in all groups did not correlate with chorioamnionitis $(\mathrm{p}=0.477)$ or $\mathrm{PE}(\mathrm{p}=0.428)$ and was tested by logistic regression. In PE AVM has previously been shown. Therefore, the PE group served as internal validation of our scoring system.

\section{Analysis of the placental weight adjusted for gestational age}

The actual placental weight of the included placentas was compared to reference data of a control cohort $(37,38)$. Placentas of the idiopathic preterm group did not show significant differences compared to the reference data of the control cohort. Reduced placental weight $(19 \%, \mathrm{p}<0.001)$ was found in the PE group, and increased placental weight $(15 \%, \mathrm{p}=0.002)$ in the chorioamnionitis group (Figure 4 and Table 3 ).

Table 3. Comparison between placental weight in the cohorts and their expected weight

\begin{tabular}{lccc} 
& Placental weight in cohort & Expected placental weight & \\
Variable & Mean \pm SD & Mean \pm SD & p \\
\hline Idiopathic preterm & $286 \pm 117$ & $276 \pm 27$ & 0.6872 \\
Chorioamnionitis (+/- funisitis) & $296 \pm 70$ & $258 \pm 32$ & $\mathbf{0 . 0 0 4 1}$ \\
PE (+/- HELLP) & $220 \pm 60$ & $273 \pm 32$ & $<\mathbf{0 . 0 0 0 1}$ \\
\hline
\end{tabular}

PE: Preeclampsia, HELLP: Hemolysis, Elevated Liver enzymes and Low Platelets. 


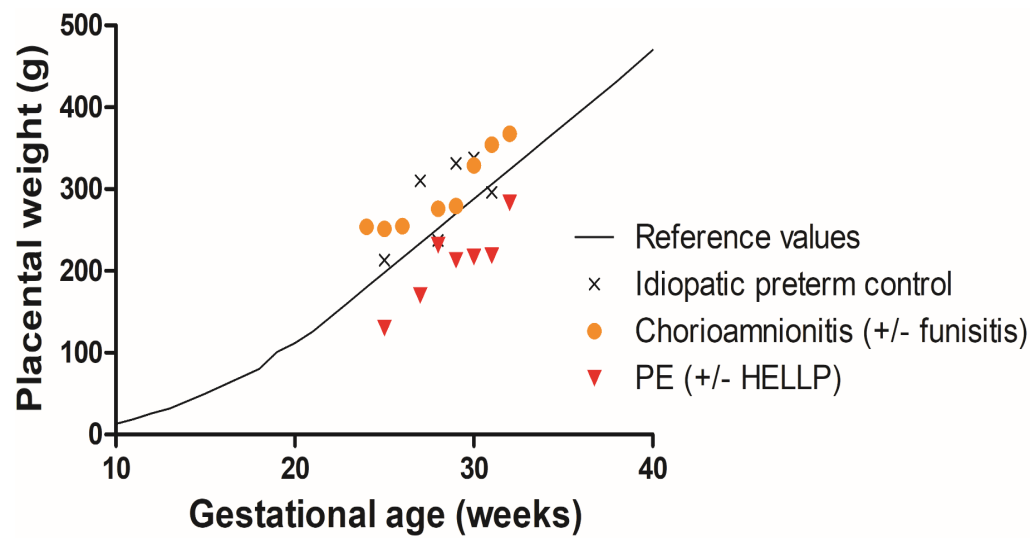

Figure 4. The mean placental weight per gestational age for each preterm group plotted against their expected placental weight.

\section{Analysis of the parameters for histological villous maturation}

\section{Idiopathic preterm vs. fully maturated}

As expected, by comparing the fully maturated term group to the idiopathic preterm group, all markers for TV maturation were found to be increased. However, the number of all villi, number of TV and the number of syncytial knots (shedded or not shedded) were not increased significantly in the term group compared to the idiopathic preterm control (Figure 5 and 6 and Table 2 and 4).

Table 4. Mean number AK, syncytial bridges and STB per high power field

\begin{tabular}{|c|c|c|c|c|c|c|c|}
\hline & $\begin{array}{c}\text { Idiopathic } \\
\text { preterm }\end{array}$ & $\begin{array}{c}\text { Chorioamnionitis } \\
\text { +/- funisitis }\end{array}$ & $\begin{array}{c}\text { PE } \\
+/ \text { - HELLP }\end{array}$ & $\begin{array}{c}\text { Non-complicated } \\
\text { Term }\end{array}$ & & & \\
\hline Expressed in mean/view & Mean \pm SD & Mean \pm SD & Mean \pm SD & Mean \pm SD & ND & $\mathbf{p}^{1}$ & $\mathbf{p}^{2}$ \\
\hline Number placentas & 24 & 41 & 35 & 15 & 1 & I & 1 \\
\hline Number AK & $0.7 \pm 0.9$ & $0.3 \pm 0.3$ & $1.7 \pm 1.3$ & $0.7 \pm 0.3$ & No & $<0.0001$ & $<0.0001$ \\
\hline Number syncytial bridges & $5.3 \pm 2.4$ & $3.5 \pm 1.4$ & $8.0 \pm 2.4$ & $8.3 \pm 2.6$ & Yes & $<0.0001$ & $<0.0001$ \\
\hline Number STB & $0.9 \pm 0.8$ & $0.7 \pm 0.6$ & $2.2 \pm 1.4$ & $0.7 \pm 0.6$ & No & $<0.0001$ & $<0.0001$ \\
\hline
\end{tabular}

PE: Preeclampsia, HELLP: Hemolysis, Elevated Liver enzymes and Low Platelets, ND: Normal distributed, AK: Apoptotic knots, STB: Shed parts of syncytiotrophoblast, Ip-value including the idiopathic preterm control, the chorioamnionitis (+/-funisitis) and the PE (+/- HELLP) groups, $2 p$-value including all groups. 

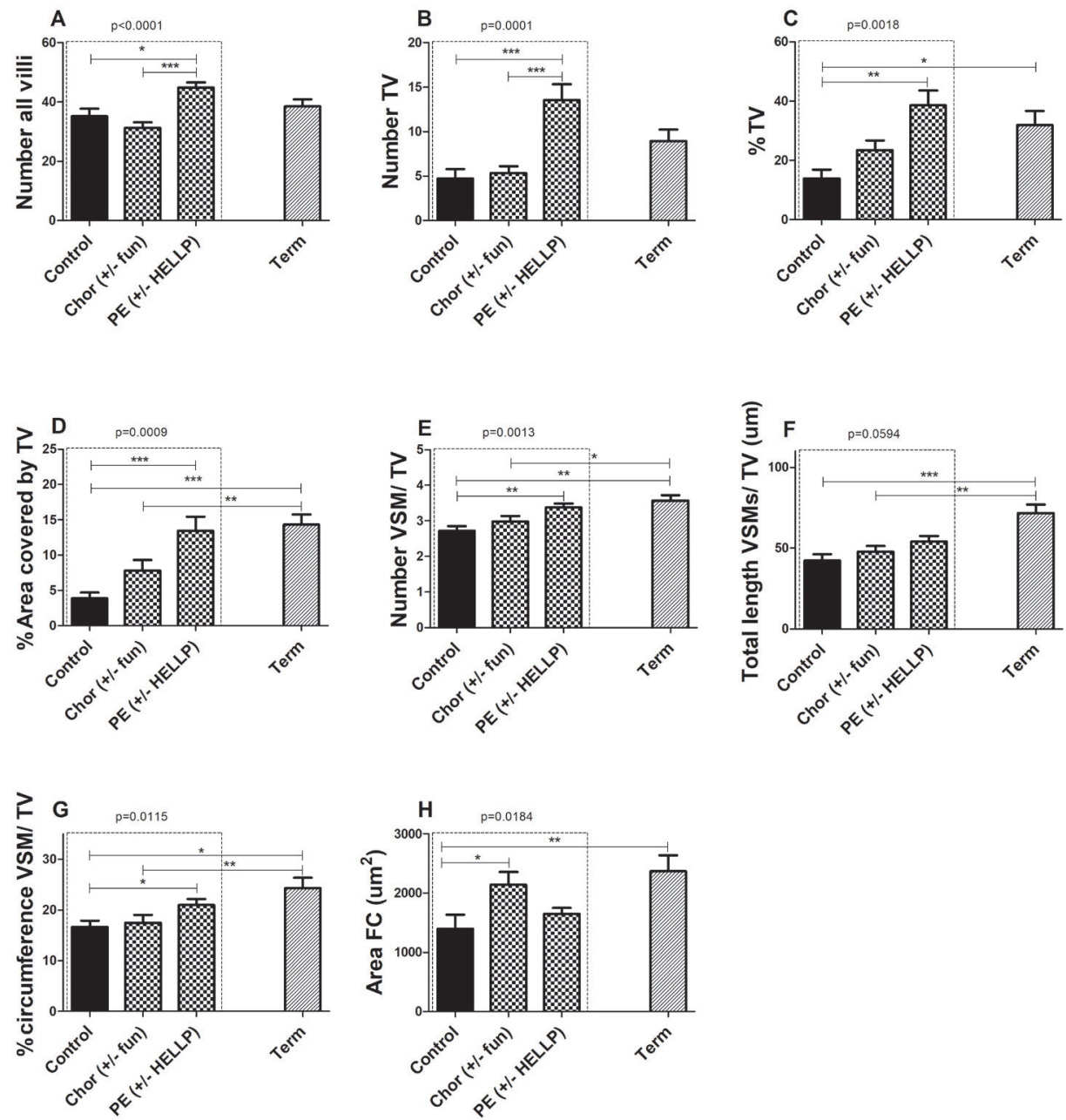

Figure 5. Number of all villi (A), Number of terminal villi (B), \% Terminal villi (C), \% Surface area covered by terminal villi (D), Number of vasculo-syncytial membranes per TV (E), Length of vasculo-syncytial membranes per TV (F), Percentage of circumference of TV covered by vasculo-syncytial membranes $(\mathrm{G})$ and Area fetal capillaries per terminal villi $(\mathrm{H})$, Villi were only counted if fully located in the high power field. Data are presented as mean with SEM. Ns: $p>0.05, * p \leq 0.05,{ }^{* *} p \leq 0.01, * * *$ $\mathrm{p} \leq 0.001$. P-values given include the 3 preterm groups. 


\section{All preterm groups}

Comparing the subgroups PE and PE + HELLP, revealed no differences for all parameters included in this study (separate data not shown). The subgroups chorioamnionitis and chorioamnionitis + funisitis also did not show differences for all parameters included in this study (separate data not shown). Therefore, the samples were analyzed as one PE +/- HELLP group and chorioamnionitis +/- funisitis group. As it is considered that clinical and histological chorioamnionitis may show differences in villous maturity, we compared 6 samples diagnosed as clinical + histological chorioamnionitis to samples with chorioamniotic histological "only". There were no differences found for all parameters included in this study (data not shown). Therefore, all samples were analyzed as one chorioamnionitis group. Except for the length of the VSMs per TV, all parameters showed a significant difference between the 3 preterm groups (Figure 5 and 6 and Table 2 and 4).
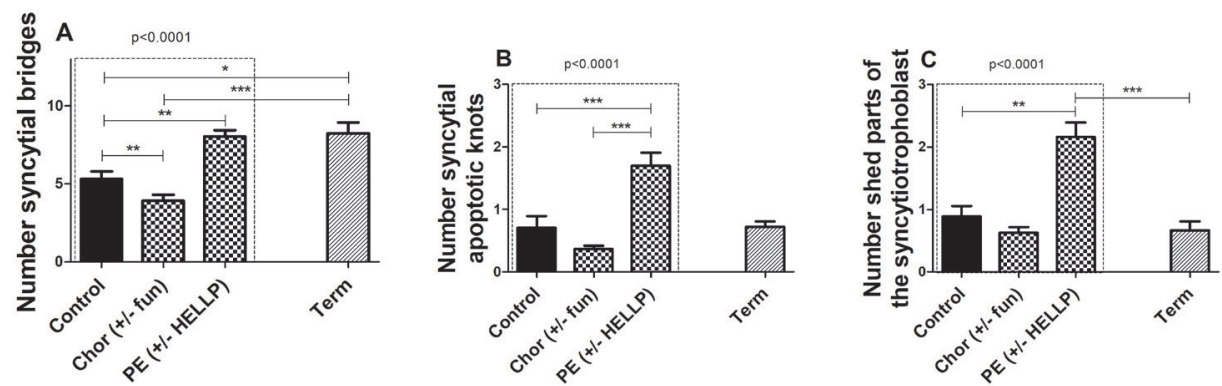

Figure 6. Mean number of total Syncytial bridges (A), Syncytial apoptotic knots (B) and Shed part of syncytiotrophoblasts (C), fully located in the high power field. Data are presented as mean with SEM. Ns: $\mathrm{p}>0.05, * \mathrm{p} \leq 0.05, * * \mathrm{p} \leq 0.01, * * * \mathrm{p} \leq 0.001$. P-values given include the 3 preterm groups.

\section{Preeclampsia}

When comparing the PE group to the preterm control group, the total number of villi was increased and there were more TV (absolute and relative to all villi) present. Furthermore, TV covered a larger area and showed more VSM covering an increased percentage of the TV circumference. The area covered by fetal capillaries was not changed. Numbers of syncytial knots and syncytial bridges were strongly increased. Numbers of all villi per view, as well as number, percentage of TV and the number of AK and shed parts of the syncytiotrophoblast were even higher in the PE group compared to the term control by $1,34,17,57$ and $69 \%$ respectively, however not statistically significant except for the number of shed parts of the syncytiotrophoblast (Figure 5 and 6). 


\section{Chorioamnionitis}

There was a significant increase in the area covered by fetal capillaries and a significant decrease in syncytial bridges (Figure 5 and 6).

Based on the binary step forward logistic regression analysis the significant parameters including the clinical parameters were weighted against each other. The number of all villi and the percentage of TV were found to be independent parameters in the prediction model of histological villous maturation (including only idiopathic preterm controls and cases of preeclampsia (+/-HELLP)) and were able to correctly predict $80 \%$ of the cases of PE within the cohort with a sensitivity of 0.82 and a specificity of 0.78 . The number of syncytial bridges and shed parts of the syncytiotrophoblast were able to correctly predict $80 \%$ of the cases of chorioamnionitis within the cohort including only idiopathic preterm controls and cases of chorioamnionitis (+/-funisitis) with a sensitivity of 0.83 and specificity of 0.75 (Table 5 and 6).

Table 5. Binary step forward logistic regression of significant parameters of histological villus maturation of preeclampsia (+/-HELLP) complicated placentas

Predicted

Preeclampsia (+/-HELLP)

\begin{tabular}{|c|c|c|c|c|c|c|}
\hline Observed & & No & Yes & Accuracy & Sensitivity & Specificity \\
\hline \multirow[t]{3}{*}{ Preeclampsia $(+/$ HELLP) } & No & 14 & 4 & 0.80 & 0.82 & 0.78 \\
\hline & Yes & 4 & 18 & & & \\
\hline & Coefficient B & Adjusted p-value & Adjusted OR & $95 \%$ CI & & \\
\hline Number all villi & 0.118 & 0.013 & 1.125 & $1.026-1.234$ & & \\
\hline TV, \% & 0.086 & 0.007 & 1.089 & $1.024-1.160$ & & \\
\hline
\end{tabular}

HELLP: Hemolysis, Elevated Liver enzymes and Low Platelet, TV: Terminal villi.

Table 6. Binary step forward logistic regression of significant parameters of histological villus maturation of chorioamnionitis (+/-funisitis) complicated placentas

\begin{tabular}{|c|c|c|c|c|c|c|}
\hline \multicolumn{7}{|c|}{ Predicted } \\
\hline Observed & & No & Yes & Accuracy & Sensitivity & Specificity \\
\hline \multirow[t]{3}{*}{ Chorioamnionitis (+/-Funisitis) } & No & 18 & 6 & 0.80 & 0.83 & 0.75 \\
\hline & Yes & 7 & 34 & & & \\
\hline & Coefficient B & Adjusted p-value & Adjusted OR & $95 \% \mathrm{CI}$ & & \\
\hline Number syncytial bridges & 0.372 & 0.010 & 1.450 & $1.092-1.925$ & & \\
\hline Number STB & 0.983 & 0.010 & 2.672 & $1.266-5.643$ & & \\
\hline
\end{tabular}

STB: Shed parts of syncytiotrophoblast. 


\section{Discussion}

By using our placental parameters, we could show that:

1. While the number of all villi and TV, as well as syncytial knotting were not significantly different between term and preterm placentas, all other parameters accounting for histological villous maturation were increased in term placentas compared to the preterm control. This may suggest that in preterm samples (30 \pm 2 weeks) branching of the placenta should be nearly completed, and histological villous maturation is then further increasing placental efficiency.

2. Concerning the PE group, all maturation markers were significantly increased compared to the preterm control group. The number of shed parts of the syncytiotrophoblast were even higher compared to the term control. This confirms that the PE group shows AVM in order to increase placental efficiency to compensate for compromised blood supply.

3. No differences between clinical and histological chorioamnionitis were found for all parameters included in this study. Furthermore, the chorioamnionitis group, including both subgroups, did not show any sign of AVM. However, the area covered by fetal capillaries was significantly increased. This may suggest that chorioamniotic placentas try to adapt to increase exchange capacity by dilation of fetal capillaries in response to the acute infection instead of developing histological AVM. This was supported by the fact that syncytial bridging was significantly decreased compared to the control group (44-46).

The number of villi, TV, percentage TV and the total length of VSM progressively increases towards the third trimester of pregnancy to ensure optimal gas exchange and nutritional supply between mother and fetus $(4,46)$. Inadequate levels of VSM and TV in the placenta can be associated with a higher incidence of neonatal asphyxia and fetal distress $(47,48)$.

Our data show that in cases complicated with PE, several maturation markers for villous characteristics are closer to the term than to the idiopathic preterm group, or even exceeded that of the term group, suggesting placental hyper maturation.

The increase in the number of villi, TV and VSM/TV in the PE group suggests an early effort to increase the efficiency of the placenta for the diffusion of gases and nutritional compounds by AVM and increased branching of the villi in PE (49). 
These data illustrate how a placenta, complicated with PE may compensate for the compromised blood supply, initiated by impaired remodeling of the spiral arteries (11). In line with our findings, earlier studies linked AVM (diagnosed as increased numbers of placental villi), to hypoxic conditions in utero, maternal vascular malperfusion, placental insufficiency and severe case of fetal growth restriction $(26,50$, 51). Recently, increased vascularization was described in PE placentas (52). This may increase efficiency of the placenta and is in line with the increase of VSM we found in our PE group.

In addition, we found a decrease in weight of placentas complicated with PE. This suggests that the switch from placental growth to an increase in efficiency via maturation takes place earlier in gestation. This may be realized via earlier differentiation of mature intermediate villi and TV from immature intermediate villi. Indeed, we found a significantly reduced number of immature intermediate villi in PE placentas (Figure 3). The resulting lower number of large stem villi might also contribute to the lower placental volume (53).

Syncytial knotting and syncytial bridging were found to be increased in the PE group. This may indicate a reaction of the placenta to the known placental hypoxic stress in PE. In agreement with our data, hypoxia as a result of venous insufficiency, was already found to be responsible for increased numbers of syncytial knots and syncytial bridges and the changes in the remodeling state of placental villi $(51,54)$. This also may be an explanation for the higher amount of cellular debris around the villi found earlier (11, 55-60).

In agreement with recent findings (34), placentas complicated with chorioamnionitis did not show such an AVM, but the area of fetal capillaries was increased per TV. Thus, placental inflammation is not reflected in placental maturation. Probably, increasing the volume of the fetal capillaries and thus the exchange volume in the placenta, may be an acute reaction to an acute infection. This may also explain the increased placental weight which might be due to extensive villous edema and fibrous connective tissue formation in response the placental inflammation, and/or to the increase in villous macrophages (Hofbauer cells) (61-63). These acute changes may explain why in most cases chorioamnionitis is not detected before birth and does not give rise to maternal symptoms and does not impair fetal growth but may induce preterm labour. Probably, the clinical symptoms are only elicited from bacteria reaching the maternal circulation in a very advanced stage. 
The gold standard of placental analysis would be a three-dimensional (3D) reconstruction of the placental villous tree. The study showed that the current gold standard using 2D-histologic identification of villous types is still valid but needs to be used with caution (64). This underlines the need for adequate markers for 2D cross section analysis. Furthermore, the interpretation of the morphology of villous maturation is subjective. Recent studies suggest that the interpretation of maturation disorders might be improved and objectified by immunohistochemistry and molecular analysis (26).

By looking at AVM, we identified a unique combination of 11 different parameters, which may assist in the analysis of villous maturation in order to diagnose placentas complicated by PE, chorioamnionitis or preterm birth. In our scoring model, we minimized the subjective steps by strict criteria and the implemented software. Moreover, the wide combination of detailed morphological parameters for placental development allowed us to:

a) show which histological adaptations take place in the placenta over ges tation

b) identify independent parameters needed for the analysis of histological villous maturation for different preterm pregnancy-related complications

c) link changes to the pathophysiology of the pathological placenta

The number of all villi and the percentage of TV were identified as independent parameters and entered the model, which was able to correctly predict $80 \%$ of the PE cases. However, when examining complicated placentas with a well-defined clinical complication, we would recommend to analyze all 11 parameters that proved AVM in the PE group, because they are useful to judge maturity in a born placenta in detail and may provide pathologists with a tool to histologically underpin the clinical diagnosis of PE. As the recurrence risk of PE and chorioamnionitis is high (65-67), it is useful to increase the knowledge of specific placental aberrations/adaptations to prevent recurrence of the pathology during a next pregnancy or develop a better/ earlier clinical management.

\section{Acknowledgements}

This study was supported by NUTRIM Graduate Program. There was no additional external funding received for this study. 


\section{References}

1. Vanterpool SF, Been JV, Houben ML, Nikkels PG, De Krijger RR, Zimmermann LJ, et al. Porphyromonas gingivalis within Placental Villous Mesenchyme and Umbilical Cord Stroma Is Associated with Adverse Pregnancy Outcome. PLoS One. 2016;11(1):e0146157.

2. Kramer BW, Kaemmerer U, Kapp M, Herbst D, Marx A, Berg D, et al. Decreased expression of angiogenic factors in placentas with chorioamnionitis after preterm birth. Pediatr Res. 2005;58(3):607-12.

3. Fisher SJ. Why is placentation abnormal in preeclampsia? Am J Obstet Gynecol. 2015;213(4 Suppl):S115-22.

4. Ruiz-Quinonez G, Reza-Lopez SA, Chavez-Corral DV, Sanchez-Ramirez B, Leal-Berumen I, Levario-Carrillo M. Placental maturity, hypertensive disorders of pregnancy and birth weight. Hypertens Pregnancy. 2014;33(2):132-44.

5. Lawn JE, Kinney M. Preterm birth: now the leading cause of child death worldwide. Sci Trans1 Med. 2014;6(263):263ed21.

6. Steegers EA, von Dadelszen P, Duvekot JJ, Pijnenborg R. Pre-eclampsia. Lancet. 2010;376(9741):631-44.

7. Saleh L, Verdonk K, Visser W, van den Meiracker AH, Danser AH. The emerging role of endothelin-1 in the pathogenesis of pre-eclampsia. Ther Adv Cardiovasc Dis. 2016;10(5):282-93.

8. Haram K, Svendsen E, Abildgaard U. The HELLP syndrome: clinical issues and management. A Review. BMC Pregnancy Childbirth. 2009;9:8.

9. Huppertz B. The Critical Role of Abnormal Trophoblast Development in the Etiology of Preeclampsia. Curr Pharm Biotechnol. 2018;19(10):771-80.

10. Smith SD, Choudhury RH, Matos P, Horn JA, Lye SJ, Dunk CE, et al. Changes in vascular extracellular matrix composition during decidual spiral arteriole remodeling in early human pregnancy. Histol Histopathol. 2016;31(5):557-71.

11. Burton GJ, Woods AW, Jauniaux E, Kingdom JC. Rheological and physiological consequences of conversion of the maternal spiral arteries for uteroplacental blood flow during human pregnancy. Placenta. 2009;30(6):473-82.

12. Burton GJ, Jauniaux E. Oxidative stress. Best Pract Res Clin Obstet Gynaecol. 2011;25(3):287-99.

13. Vangrieken P, Al-Nasiry S, Janssen GMJ, Weseler AR, Spaanderman ME, Bast A, et al. The direct and sustained consequences of severe placental hypoxia on vascular contractility. PLoS One. 2018;13(8):e0202648.

14. Borzychowski AM, Sargent IL, Redman CW. Inflammation and pre-eclampsia. Semin Fetal Neonatal Med. 2006;11(5):309-16.

15. Yoon BH, Romero R, Moon JB, Shim SS, Kim M, Kim G, et al. Clinical 
significance of intra-amniotic inflammation in patients with preterm labor and intact membranes. Am J Obstet Gynecol. 2001;185(5):1130-6.

16. Eschenbach DA. Ureaplasma urealyticum and premature birth. Clin Infect Dis. 1993;17 Suppl 1:S100-6.

17. Robbins JR, Bakardjiev AI. Pathogens and the placental fortress. Current opinion in microbiology. 2012;15(1):36-43.

18. Crocker I, Tanner O, Myers J, Bulmer J, Walraven G, Baker P. Syncytiotrophoblast degradation and the pathophysiology of the malaria-infected placenta. Placenta. 2004;25(4):273-82.

19. Williams GJ, Hodson EH, Isaacs D, Craig JC. Diagnosis and management of urinary tract infection in children. J Paediatr Child Health. 2012;48(4):296-301.

20. Tita AT, Andrews WW. Diagnosis and management of clinical chorioamnionitis. Clin Perinatol. 2010;37(2):339-54.

21. Romero R, Miranda J, Chaiworapongsa T, Korzeniewski SJ, Chaemsaithong $\mathrm{P}$, Gotsch F, et al. Prevalence and clinical significance of sterile intra-amniotic inflammation in patients with preterm labor and intact membranes. Am J Reprod Immunol. 2014;72(5):458-74.

22. Redline RW. Classification of placental lesions. Am J Obstet Gynecol. 2015;213(4 Suppl):S21-8.

23. Coleman SJ, Gerza L, Jones CJ, Sibley CP, Aplin JD, Heazell AE. Syncytial nuclear aggregates in normal placenta show increased nuclear condensation, but apoptosis and cytoskeletal redistribution are uncommon. Placenta. 2013;34(5):44955.

24. Loukeris K, Sela R, Baergen RN. Syncytial knots as a reflection of placental maturity: reference values for 20 to 40 weeks' gestational age. Pediatric and Developmental Pathology. 2010;13(4):305-9.

25. Kaufmann P, Huppertz B. Tenney-Parker changes and apoptotic versus necrotic shedding of trophoblast in normal pregnancy. Pre-eclampsia: etiology and clinical practice. 2007:152.

26. Turowski G, Vogel M. Re-view and view on maturation disorders in the placenta. APMIS. 2018;126(7):602-12.

27. Huppertz B, Kadyrov M, Kingdom JCP. Apoptosis and its role in the trophoblast. American Journal of Obstetrics and Gynecology. 2006;195(1):29-39.

28. Burton GJ, Jones CJ. Syncytial knots, sprouts, apoptosis, and trophoblast deportation from the human placenta. Taiwan J Obstet Gynecol. 2009;48(1):28-37.

29. Mayhew TM. Villous trophoblast of human placenta: a coherent view of its turnover, repair and contributions to villous development and maturation. Histol Histopathol. 2001;16(4):1213-24.

30. Sultana Z, Maiti K, Aitken J, Morris J, Dedman L, Smith R. Oxidative stress, 
placental ageing-related pathologies and adverse pregnancy outcomes. Am J Reprod Immunol. 2017;77(5).

31. Sultana Z, Maiti K, Dedman L, Smith R. Is there a role for placental senescence in the genesis of obstetric complications and fetal growth restriction? Am J Obstet Gynecol. 2018;218(2S):S762-S73.

32. Maiti K, Sultana Z, Aitken RJ, Morris J, Park F, Andrew B, et al. Evidence that fetal death is associated with placental aging. Am J Obstet Gynecol. 2017;217(4):441 e1- e14.

33. Gomez-Lopez N, Romero R, Plazyo O, Schwenkel G, Garcia-Flores V, Unkel R, et al. Preterm labor in the absence of acute histologic chorioamnionitis is characterized by cellular senescence of the chorioamniotic membranes. Am J Obstet Gynecol. 2017;217(5):592 e1- e17.

34. Christians JK, Grynspan D. Placental villous hypermaturation is associated with improved neonatal outcomes. Placenta. 2019;76:1-5.

35. Smit AL, Been JV, Zimmermann LJ, Kornelisse RF, Andriessen P, Vanterpool SF, et al. Automated auditory brainstem response in preterm newborns with histological chorioamnionitis. J Matern Fetal Neonatal Med. 2015;28(15):1864-9.

36. Redline RW, Faye-Petersen O, Heller D, Qureshi F, Savell V, Vogler C, et al. Amniotic infection syndrome: nosology and reproducibility of placental reaction patterns. Pediatr Dev Pathol. 2003;6(5):435-48.

37. Benirschke K, Driscoll SG. The pathology of the human placenta: Springer; 1967.

38. Castellucci M kP. Pathology of the human placenta. 5th Edition Ed: Benirschke K, Kaufmann P, Baergen R 2006. 2006:506.

39. Loukeris K, Sela R, Baergen RN. Syncytial knots as a reflection of placental maturity: reference values for 20 to 40 weeks' gestational age. Pediatr Dev Pathol. 2010;13(4):305-9.

40. Jones CJ, Fox H. Syncytial knots and intervillous bridges in the human placenta: an ultrastructural study. J Anat. 1977;124(Pt 2):275-86.

41. Johansen M, Redman CW, Wilkins T, Sargent IL. Trophoblast deportation in human pregnancy--its relevance for pre-eclampsia. Placenta. 1999;20(7):531-9.

42. Askelund KJ, Chamley LW. Trophoblast deportation part I: review of the evidence demonstrating trophoblast shedding and deportation during human pregnancy. Placenta. 2011;32(10):716-23.

43. Landis JR, Koch GG. The measurement of observer agreement for categorical data. biometrics. 1977:159-74.

44. Taki A, Abe M, Komaki M, Oku K, Iseki S, Mizutani S, et al. Expression of angiogenesis-related factors and inflammatory cytokines in placenta and umbilical vessels in pregnancies with preeclampsia and chorioamnionitis/funisitis. Congenit 
Anom (Kyoto). 2012;52(2):97-103.

45. Naeye RL, Maisels MJ, Lorenz RP, Botti JJ. The clinical significance of placental villous edema. Pediatrics. 1983;71(4):588-94.

46. Mihu CM, Susman S, Rus Ciuca D, Mihu D, Costin N. Aspects of placental morphogenesis and angiogenesis. Rom J Morphol Embryol. 2009;50(4):549-57.

47. Fox $\mathrm{H}$. The incidence and significance of vasculo-syncytial membranes in the human placenta. J Obstet Gynaecol Br Commonw. 1967;74(1):28-33.

48. Sankar KD, Bhanu PS, Kiran S, Ramakrishna BA, Shanthi V. Vasculosyncytial membrane in relation to syncytial knots complicates the placenta in preeclampsia: a histomorphometrical study. Anat Cell Biol. 2012;45(2):86-91.

49. Kaufmann P, Bruns U, Leiser R, Luckhardt M, Winterhager E. The fetal vascularisation of term human placental villi. II. Intermediate and terminal villi. Anatomy and embryology. 1985;173(2):203-14.

50. Yaguchi C, Itoh H, Tsuchiya KJ, Furuta-Isomura N, Horikoshi Y, Matsumoto $\mathrm{M}$, et al. Placental pathology predicts infantile physical development during first 18 months in Japanese population: Hamamatsu birth cohort for mothers and children (HBC Study). PLoS One. 2018;13(4):e0194988.

51. Garcia-Honduvilla N, Ortega MA, Asunsolo A, Alvarez-Rocha MJ, Romero B, De Leon-Luis J, et al. Placentas from women with pregnancy-associated venous insufficiency show villi damage with evidence of hypoxic cellular stress. Hum Pathol. 2018;77:45-53.

52. Eddy AC, Bidwell GL, 3rd, George EM. Pro-angiogenic therapeutics for preeclampsia. Biol Sex Differ. 2018;9(1):36.

53. Castellucci M, Scheper M, Scheffen I, Celona A, Kaufmann P. The development of the human placental villous tree. Anatomy and embryology. 1990;181(2):11728.

54. Ortega MA, Asunsolo A, Alvarez-Rocha MJ, Romero B, De Leon-Luis J, Alvarez-Mon M, et al. Remodelling of collagen fibres in the placentas of women with venous insufficiency during pregnancy. Histol Histopathol. 2018;33(6):567-76. 55. Cindrova-Davies T, Spasic-Boskovic O, Jauniaux E, Charnock-Jones DS, Burton GJ. Nuclear factor-kappa B, p38, and stress-activated protein kinase mitogen-activated protein kinase signaling pathways regulate proinflammatory cytokines and apoptosis in human placental explants in response to oxidative stress: effects of antioxidant vitamins. Am J Pathol. 2007;170(5):1511-20.

56. Myatt L, Cui X. Oxidative stress in the placenta. Histochem Cell Biol. 2004;122(4):369-82.

57. Huppertz B, Kingdom J, Caniggia I, Desoye G, Black S, Korr H, et al. Hypoxia favours necrotic versus apoptotic shedding of placental syncytiotrophoblast into the maternal circulation. Placenta. 2003;24(2-3):181-90. 
58. Sharp AN, Heazell AE, Crocker IP, Mor G. Placental apoptosis in health and disease. Am J Reprod Immunol. 2010;64(3):159-69.

59. Arad A, Nammouz S, Nov Y, Ohel G, Bejar J, Vadasz Z. The Expression of Neuropilin-1 in Human Placentas From Normal and Preeclamptic Pregnancies. Int J Gynecol Pathol. 2017;36(1):42-9.

60. Cindrova-Davies T. Gabor Than Award Lecture 2008: pre-eclampsia - from placental oxidative stress to maternal endothelial dysfunction. Placenta. 2009;30 Suppl A:S55-65.

61. Pankuch GA, Appelbaum PC, Lorenz RP, Botti JJ, Schachter J, Naeye RL. Placental microbiology and histology and the pathogenesis of chorioamnionitis. Obstetrics \& Gynecology. 1984;64(6):802-6.

62. Naeye RL, Maisels MJ, Lorenz RP, Botti JJ. The clinical significance of placental villous edema. Pediatrics. 1983;71(4):588-93.

63. Tang Z, Abrahams VM, Mor G, Guller S. Placental Hofbauer cells and complications of pregnancy. Ann N Y Acad Sci. 2011;1221:103-8.

64. Haeussner E, Aschauer B, Burton GJ, Huppertz B, Edler von Koch F, Muller-Starck J, et al. Does 2D-Histologic identification of villous types of human placentas at birth enable sensitive and reliable interpretation of 3D structure? Placenta. 2015;36(12):1425-32.

65. Roberts CL, Seeho SK, Algert CS, Ford JB. Early onset preeclampsia: using population data to assess recurrence risk and adverse pregnancy outcomes. International Journal of Population Data Science. 2017;1(1).

66. Dypvik J, Larsen S, Haavaldsen C, Jukic AM, Vatten LJ, Eskild A. Placental weight in the first pregnancy and risk for preeclampsia in the second pregnancy: A population-based study of 186859 women. European Journal of Obstetrics \& Gynecology and Reproductive Biology. 2017;214:184-9.

67. Laibl VR, Sheffield JS, Roberts S, McIntire DD, Wendel GD. Recurrence of clinical chorioamnionitis in subsequent pregnancies. Obstetrics \& Gynecology. 2006;108(6):1493-7. 



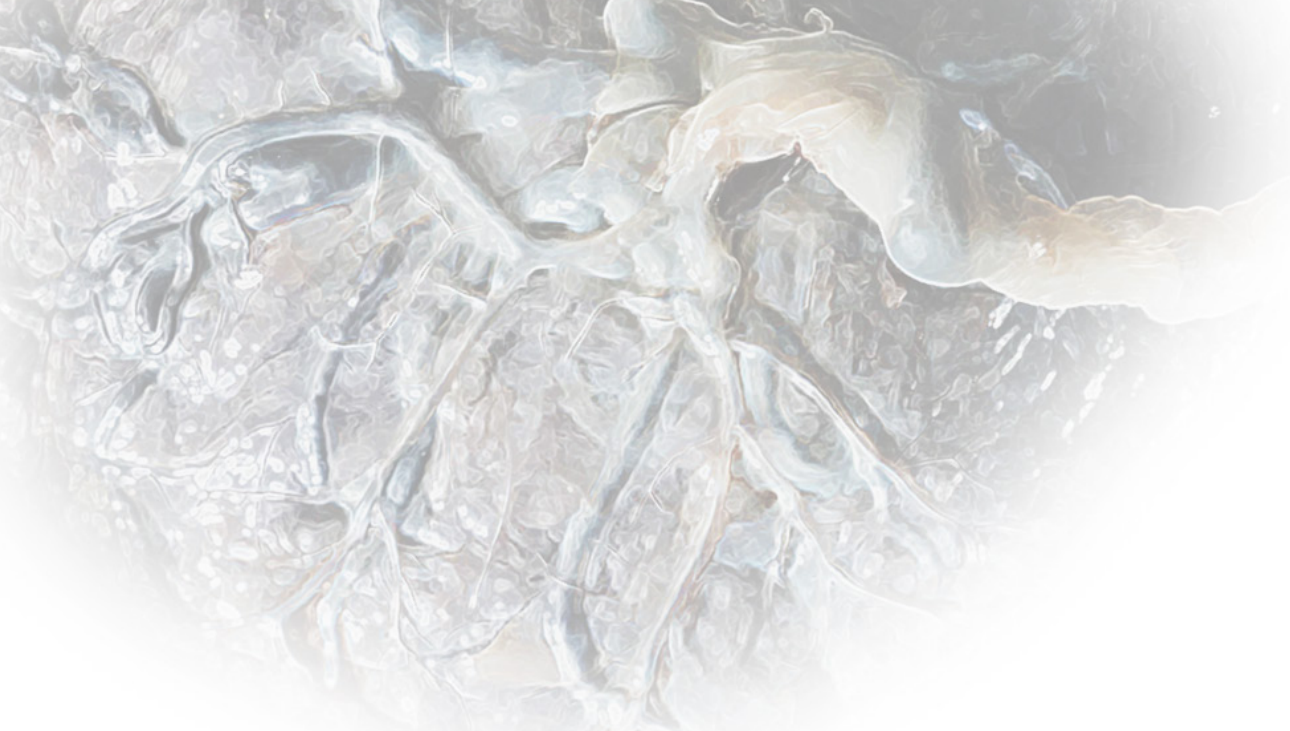




\section{Chapter 3}

\section{P lacental mitochondrial abnormalities in preeclampsia}

Philippe Vangrieken, Salwan Al-Nasiry, Aalt Bast, Pieter A. Leermakers, Christy B. M. Tulen, Paul M. H. Schiffers, Frederik J. van Schooten, Alex H. V. Remels

Submitted 


\section{Abstract}

\section{Introduction}

Preeclampsia complicates 5-8\% of all pregnancies worldwide and, although its pathophysiology remains obscure, placental oxidative stress and mitochondrial abnormalities are considered to play a key role. Mitochondrial abnormalities in preeclamptic placentae have been described but the extent to which mitochondrial content and the molecular pathways controlling this (mitochondrial biogenesis and mitophagy) are affected in preeclamptic placentae is unknown.

\section{Methods}

Therefore, in preeclamptic $(\mathrm{n}=12)$ and control $(\mathrm{n}=11)$ placentae, we comprehensively assessed multiple indices of placental antioxidant status, mitochondrial content, mitochondrial biogenesis, mitophagy and mitochondrial fusion and fission. In addition, we also explored gene expression profiles related to inflammation and apoptosis.

\section{Results}

Preeclamptic placentae were characterized by higher levels of oxidized glutathione, a higher total antioxidant capacity and higher mRNA levels of the mitochondrial-located antioxidant enzyme manganese-dependent superoxide dismutase 2 compared to controls. Furthermore, mitochondrial content was significantly lower in preeclamptic placentae, which was accompanied by an increased abundance of key constituents of glycolysis. Moreover, mRNA and protein levels of key molecules involved in the regulation of mitochondrial biogenesis were lower in preeclamptic placentae while the abundance of constituents of the mitophagy, autophagy and mitochondrial fission machinery was higher compared to controls. In addition, we found evidence for activation of apoptosis and inflammation in preeclamptic placentae.

\section{Conclusion}

This study is the first to comprehensively demonstrate abnormalities at the level of the mitochondrion and the molecular pathways controlling mitochondrial content/ function in preeclamptic placentae. These aberrations may well contribute to the pathophysiology of preeclampsia by upregulating placental inflammation, oxidative stress and apoptosis. 


\section{Introduction}

Preeclampsia (PE) is a pregnancy disorder that occurs in $5-8 \%$ of all pregnancies and has potentially devastating consequences for both mother and fetus. The clinical spectrum of $\mathrm{PE}$ ranges from relatively mild to life-threatening and $\mathrm{PE}$ is estimated to be responsible for 70,000-80,000 maternal deaths and 500,000 perinatal deaths worldwide every year (1). PE is not simply de novo onset of hypertension and proteinuria after 20 weeks of gestation, but rather a syndrome involving multiple organs resulting in end-organ damage in terms of cardiovascular, respiratory, central nervous, renal, and hepatic systems $(2,3)$. Pre-term delivery is often the only definite treatment for PE, which is associated with adverse short- and long-term health outcomes in the offspring including a high prevalence of subsequent endocrine and metabolic diseases in children $(4,5)$. The pathophysiology of PE remains enigmatic and except for delivery, no curative treatment currently exists. There is substantial evidence that poor invasion of extra-villous trophoblasts into the uterine wall and spiral arteries leads to a perturbed utero-placental circulation. Although the underlying cellular and molecular mechanisms involved remain unknown, it is believed that, as pregnancy progresses, this aberrant placental perfusion induces further damage and elicits oxidative stress in the placenta, which contributes to the ongoing development of the disease (6-8).

It is well-known that disturbed placental perfusion, as observed in patients suffering from PE, can trigger the production of reactive oxygen species (ROS) $(9,10)$. Cellular responses in the placenta to ROS and ROS-induced damage in PE includes the activation/ upregulation of several antioxidant systems (e.g. superoxide dismutase (SOD) and catalase), as well as apoptosis of trophoblasts (11). Importantly, oxidative stress does not only trigger trophoblast cell death, but also accelerates trophoblast turnover and its secretome into the maternal circulation. These changes in the trophoblast secretome include an increased release of vasoactive factors activating the angiotensin-I and endothelin-1 receptor as well as an enhanced release of inflammatory cytokines and chemokines $(2,12-14)$. Alterations in secretion of these placental factors into the maternal circulation induce inflammation, endothelial dysfunction, and PE-like symptoms including hypertension (2). Although the presence of placental oxidative stress and its contribution to the PE pathophysiology are commonly acknowledged, the sources of ROS and the exact underlying processes of its formation remain obscure. Clinical trials using vitamin $\mathrm{C}$ and $\mathrm{E}$ for the treatment of $\mathrm{PE}$ have been unsuccessful, illustrating that systemically acting antioxidants to restore redox imbalance have no clinical relevance (15). Similar to observations in other clinical fields, research is directed nowadays into finding more specific redox-targets with 
the purpose of combating oxidative stress in a more selective way, as for instance in the field of respiratory diseases (16).

Recently, mitochondria, a main intracellular source of ROS, have gained more interest as a potential novel therapeutic target in PE. In a recent study, administration of MitoQ, a mitochondrially targeted antioxidant, improved foetal outcomes, including birth weight and developmental programming of cardiovascular diseases, in a rodent model (chronic placental hypoxia) for PE (17-20). However, whether mitochondrial dysfunction is present in PE placentae and how this contributes to the development of oxidative stress, and the pathophysiology of this disease is still unknown.

Therefore, in this study, using PE and control placentae, we comprehensively assessed not only the presence of oxidative stress and the status of well-known cellular antioxidant systems, but also investigated, in detail, multiple indices of mitochondrial health. This included key constituents of metabolic pathways including oxidative phosphorylation, fatty acid $\beta$-oxidation and glycolysis. Furthermore, to portray molecular mechanisms that control mitochondrial content and function, we also assessed key regulators of mitochondrial biogenesis, as well as constituents of the mitophagy/autophagy, and mitochondrial fusion and fission machinery. 


\section{Materials and Methods}

\section{Clinical subject characteristics}

Approval was obtained from the Medical Ethics Committee Maastricht University Medical Center+ and all procedures on placentae were performed in accordance with the guidelines for experiments on human material (Maastricht University Medical Center+, Maastricht, the Netherlands, No. 16-4-047). Oral informed consent was obtained from participants prior to the inclusion of the placenta.

Human term placentae $(n=11)$ and PE-complicated placentae $(n=12)$ were collected from in total 23 women by the Department of Obstetrics and Gynecology at the Maastricht University Medical Center + . PE was diagnosed based on the International Society for the Study of Hypertension in Pregnancy (ISSHP) criteria as de novo hypertension with a systolic blood pressure $\geq 140 \mathrm{~mm} \mathrm{Hg}$ and/or diastolic blood pressure $\geq 90 \mathrm{~mm} \mathrm{Hg}$ in 2 repeated measurements (at least 6 hours apart) and the co-occurrence of proteinuria ( $\geq 0.3 \mathrm{~g} / 24 \mathrm{~h}$ or $\geq 2+$ on dipstick analysis) occurring $>20$ weeks of gestation in previously normotensive women or when proteinuria developed $>20$ weeks of gestation in women with pre-existing hypertension. Exclusion criteria for control pregnancies were hypertension (pre-existing or onset during pregnancy) and/ or proteinuria, also as defined by ISSHP, chromosomal anomalies, multiple gestation and autoimmune diseases. Intra uterine growth restriction (IUGR) was defined as neonatal birthweight below the 10th centile according Dutch perinatal registry curves. Clinical subject characteristics are presented in Table 1.

Table 1: Clinical subject characteristics

\begin{tabular}{lccc} 
& $\begin{array}{c}\text { Control } \\
(\mathbf{n = 1 1})\end{array}$ & $\begin{array}{c}\text { Preeclampsia } \\
(\mathbf{n = 1 2})\end{array}$ & Significance \\
\hline Gestational age at delivery (Weeks) & $39 \pm 1$ & $33 \pm 3$ & $* * *$ \\
Maternal age (Years) & $30 \pm 4$ & $30 \pm 5$ & Ns \\
Maternal BMI (Kg/m²) & $25 \pm 6$ & $24 \pm 4$ & Ns \\
Neonatal birth weight (Kg) & $3.4 \pm 0.4$ & $2.4 \pm 0.9$ & $* *$ \\
Neonatal sex (male, \%) & 64 & 58 & - \\
IUGR (\%) & 0 & 50 & - \\
Mode of delivery (CS, \%) & 55 & 58 & - \\
\hline
\end{tabular}

Data presented as mean \pm standard deviation. BMI: Body mass index, IUGR: Intrauterine growth restriction, CS: Caesarean section, Ns: Non-significant. Ns: $p>0.05, * * p \leq 0.01, * * * p \leq 0.001$. 


\section{Tissue collection}

Placental biopsies $\left(<1 \mathrm{~cm}^{2}\right)$ were collected immediately after delivery from the paracentral region of the placenta at the maternal side. The basal plate of the specimen was removed and the remaining tissue was rinsed in a HEPES solution $(\mathrm{NaCl} 143.3$ $\mathrm{mM}, \mathrm{KCl} 4.7 \mathrm{mM}, \mathrm{MgSO}_{4} 1.2 \mathrm{mM}, \mathrm{KH}_{2} \mathrm{PO}_{4} 1.2 \mathrm{mM}, \mathrm{CaCl}_{2} 2.5 \mathrm{mM}$, Glucose 5.5 $\mathrm{mM}$, and HEPES $15 \mathrm{mM}$ ), snap-frozen in liquid nitrogen, crushed by a mortar while frozen and stored at $-80{ }^{\circ} \mathrm{C}$ until use.

\section{Preparation of the lysates}

Approximately $40 \mathrm{mg}$ of powdered placental tissue was homogenized for $10 \mathrm{sec}$ at maximal speed with a hand held PRO Scientific Bio-Gen PRO200 homogenizer in $800 \mu \mathrm{KPE}$ lysis buffer $\left(13 \mathrm{mM} \mathrm{KH} \mathrm{PO}_{4}, 68 \mathrm{mM} \mathrm{K} 2 \mathrm{HPO} 4,9 \mathrm{mM}\right.$ EDTA, and $1 \%$ Triton X-100) for antioxidant enzyme activity assays or in $800 \mu 1$ SET buffer (250 mM sucrose, $2 \mathrm{mM}$ EDTA, $10 \mathrm{mM}$ Tris, $\mathrm{pH}$ 7.4) for metabolic enzyme activity assays. KPE homogenates were subsequently centrifuged at $20,000 \mathrm{x}$ g for 10 min at $4{ }^{\circ} \mathrm{C}$. Subsequently, lysates $(300 \mu \mathrm{l})$ were stored at $-80^{\circ} \mathrm{C}$ for assessment of Trolox Equivalent Antioxidant Capacity (TEAC). The remaining lysate was mixed with $2.6 \%$ bovine serum albumin (BSA) $(1: 1)$ and stored at $-80^{\circ} \mathrm{C}$ for determination of glutathione disulfide/glutathione (GSSG/GSH) levels. SET homogenates were snap-frozen in liquid nitrogen, defrosted, incubated on ice for $30 \mathrm{~min}$ and subsequently centrifuged at $20,000 \times \mathrm{g}$ for $2 \mathrm{~min}$ at $4{ }^{\circ} \mathrm{C} .5 \% \mathrm{BSA}$ was added to the lysate (1:4) and stored at $-80{ }^{\circ} \mathrm{C}$ for the assessment of citrate synthase (CS), $\beta$-hydroxyacyl-CoA dehydrogenase (HADH) and phosphofructokinase (PFK) activity. For DNA and RNA isolation, $40 \mathrm{mg}$ of powdered placental tissue was homogenized in $1 \mathrm{ml}$ Trizol reagent (Invitrogen Corp.) and further processed according to the manufacturer's protocol (Catalog Number 15596026 and 15596018, Invitrogen ${ }^{\mathrm{TM}}$, USA). For generation of whole cell lysates (for western blot analysis), $40 \mathrm{mg}$ of powdered placental tissue was homogenized in $800 \mu \mathrm{IP}$ lysis buffer $(50 \mathrm{mM}$ Tris, $150 \mathrm{mM}$ $\mathrm{NaCl}, 10 \%$ glycerol, $0.5 \%$ Nonidet P40, 1 mM EDTA, $1 \mathrm{mM} \mathrm{Na} 3 \mathrm{VO} 4,5 \mathrm{mM} \mathrm{NaF}$, $10 \mathrm{mM} \beta$-glycerophasphate, $1 \mathrm{mM} \mathrm{Na}_{4} \mathrm{O}_{7} \mathrm{P}_{2}, 1 \mathrm{mM}$ DTT, $10 \mu \mathrm{g} / \mu \mathrm{l}$ leupeptin, $1 \%$ apropeptin, $1 \mathrm{mM}$ PMSF, pH 7.4) using the homogenizer for $20 \mathrm{sec}$ at maximal speed. Lysates were incubated for $30 \mathrm{~min}$ on ice and centrifuged at 20,000 x g for $30 \mathrm{~min}$ at $4{ }^{\circ} \mathrm{C}$. Lysates were aliquoted $(1 \mu \mathrm{g} / \mu \mathrm{l})$ in Laemmli buffer buffer $(0.25 \mathrm{M}$ Tris- $\mathrm{HCl}$, $8 \%$ (w/v) SDS, $40 \%$ (v/v) glycerol, 0.4 M DTT, 0.04\% (w/v) Bromphenol Blue, $\mathrm{pH}$ 6.8) and boiled for $5 \mathrm{~min}$ at $95{ }^{\circ} \mathrm{C}$. Protein concentrations of whole cell lysates and enzyme activity lysates were determined using the PierceTM BCA Protein Assay kit according to the manufacturer's protocol (Pierce Chemical Co., Rockford, IL). 


\section{Glutathione disulfide/glutathione (GSSG/GSH) levels}

The GSH-assay was performed for the determination of the levels of GSH + GSSG and GSSG. First, GSH $(0.1-10 \mu \mathrm{M})$ and GSSG $(0.1-5 \mu \mathrm{M})$ standards were prepared in a KPE buffer and 1.3\% 5-sulfosalicylic acid. GSSG standards and samples were diluted 1:10 with 2-vinylpyridine, incubated and mixed for $1 \mathrm{~h}$ to form a stable complex with GSH, preventing it from participating in the enzymatic recycling reaction with glutathione reductase. Reactions were set up in a 96 well plate and $50 \mu 1$ of sample was loaded in duplicate. Reactions were initiated by adding $100 \mu 10.8 \mathrm{mM}$ NADPH/0.6 mM DTNB 1:1 and $4 \mathrm{U} / \mathrm{ml} \mathrm{GSSG}$ reductase to the samples. Color development of samples and standards was recorded kinetically for $3 \min$ in 9 reads at $412 \mathrm{nM}$ resulting in GSH + GSSG and GSH slope values. Activity was corrected for total protein content of the samples and expressed in $\mathrm{nM} / \mathrm{mg}$ protein/ $\mathrm{min}$.

\section{Trolox Equivalent Antioxidant Capacity (TEAC)}

First, a 5 mM 2,2'-azino-bis(3-ethylbenzothiazoline-6-sulphonic acid) (ABTS) solution was prepared in $145 \mathrm{mM}$ sodium phosphate buffer ( $\mathrm{pH}$ 7.4). Then, an (ABTS ${ }^{-}$) solution was prepared by adding $10 \mu \mathrm{l} 1 / 100$ horseradish peroxidase (HRP) and 10 $\mu \mathrm{l}$ of $2 \mathrm{mM} \mathrm{H}_{2} \mathrm{O}_{2}$ solution and diluted in an ABTS solution to a final absorbance of $0.70 \pm 0.02$ at $734 \mathrm{~nm}$ at $37^{\circ} \mathrm{C}$. Deproteinization of samples was performed by adding $10 \%$ trichloroacetic acid (TCA) $(1: 1)$ to the samples. For measuring antioxidant capacity, $50 \mu \mathrm{l}$ of the lysate was mixed with $950 \mu \mathrm{lBCS} \mathrm{AB}^{-}$solution at $37^{\circ} \mathrm{C}$ for 5 min and absorbance was measured at $734 \mathrm{~nm}$ and compared to the absorbance of an ABTS $^{-}{ }^{-}$solution without sample. Absorbance was corrected for total protein content.

\section{Citrate synthase (CS) activity}

As previously described (CS; EC 2.3.3.1) (21), a reaction mix was set up in a 96 well plate in duplicate containing $5 \mu \mathrm{l}$ undiluted sample, $200 \mu 1$ reagent containing Tris $(100 \mathrm{mM})$, DTNB $(0.1 \mathrm{mM})$, acetyl-coenzym A $(0.3 \mathrm{mM})$. The reaction was started with $5 \mu \mathrm{l}$ start reagent containing oxaloacetic acid $(25 \mathrm{mM})$. Enzyme activity was monitored at $412 \mathrm{~nm}\left(37^{\circ} \mathrm{C}\right)$ and corrected for total protein content.

\section{B-hydroxyacyl-CoA dehydrogenase (HADH) activity}

As previously described (HADH; EC 1.1.1.35) (22), a reaction mix was set up in a 96 well plate in duplicate containing $10 \mu 1$ undiluted sample, $100 \mu 1$ reagent containing NADH $(1.1 \mathrm{mM})$, tetrapotassium pyrophosphate $(100 \mathrm{mM})$. The reaction was started with $10 \mu$ acetoacetyl-CoA $(2.4 \mathrm{mM})$. Enzyme activity was kinetically-monitored at $340 \mathrm{~nm}\left(37^{\circ} \mathrm{C}\right)$ and corrected for total protein content. 


\section{Phosphofructokinase (PFK) activity}

A reaction mix was set up in a 96 well plate in duplicate containing $20 \mu 1$ undiluted sample, $100 \mu \mathrm{l}$ reagent containing Tris Base $(49.6 \mathrm{mM}), \mathrm{MgCl}_{2} .6 \mathrm{H}_{2} \mathrm{O}(7.4 \mathrm{mM})$, $\mathrm{KCl}(3.2 \mathrm{mM}), \mathrm{KCN}(384.6 \mu \mathrm{M})$, ATP (3.0 mM), DTT (1.5 mM), NADH (0.3 mM), aldolase (0.019 U), glycerol-3-phosphate dehydrogynase (0.019 U) and triose phosphate, isomerase $(0.019 \mathrm{U}), \mathrm{pH}$ 8.0. The reaction was started with fructose-6-phosphate $(35.9 \mathrm{mM})$ in Tris buffer $(49.6 \mathrm{mM}), \mathrm{pH}$ 8.0. Enzyme activity was monitored at $340 \mathrm{~nm}\left(37^{\circ} \mathrm{C}\right)$ and corrected for total protein content.

\section{Quantitive real-time PCR}

$4.4 \mu \mathrm{l}$ of 1:50 diluted cDNA was used for quantitative PCR amplification using target specific primers (Supplementary Table 1) and 2X SensimixTM SYBER \& \& Fluorescein mix (Bioline, Alphen aan de Rijn, the Netherlands) on a LightCycler480 384-wells PCR machine (Roche, Almere, the Netherlands). Specificity of PCR amplification was checked by melt curve analysis. Expression levels of genes of interest were corrected using a normalization factor calculated based on the expression of 2 different housekeeping genes (Cyclophilin A and Ribosomal Protein L13a (RPL13A)), which were found to be most stable from a selection of 3 genes by using the GeNorm software (Primerdesign, Southamton, USA). The list of primers can be found in Supplementary Table 1.

\section{Mitochondrial DNA (mtDNA) copy number}

$4.4 \mu \mathrm{l}$ of 1:25 diluted DNA was used for qPCR as described above, using mitochondrial DNA (mtDNA, cytochrome C oxidase subunit 2 (COXII)) and genomic DNA (gDNA, RPL13A) - specific primers (Supplementary Table 1). mtDNA/gDNA ratio was determined by dividing the relative quantity of mtDNA by the relative quantity of gDNA.

\section{Western Blotting}

$10 \mu \mathrm{g}$ of protein was run through a Criterion XT 4-12 or 12\% Bis-Tris gel (Bio-Rad, Veenendaal, the Netherlands) in 1x MES running buffer (Bio-Rad, Veenendaal, the Netherlands) at 100 volts, and was subsequently blotted on a Nitrocellulose membrane (Bio-Rad Laboratories B.V., Veenendaal, the Netherlands) by electroblotting. At least two protein ladders were loaded on each gel (Precision Plus Protein ${ }^{\mathrm{TM}}$ All Blue Standards \#161-0373, Bio-Rad Laboratories, Veenendaal, the Netherlands). Membranes were stained with $0.2 \%$ PonceauS in $1 \%$ acetic acid (Sigma-Aldrich, Zwijndrecht, the Netherlands) for $5 \mathrm{~min}$, washed with milliQ and imaged using the Amersham $^{\mathrm{TM}}$ Imager 600 (GE Healthcare, Eindhoven, the Netherlands) to quantify total protein content as correction for gel-loading. Membranes were blocked for $1 \mathrm{~h}$ 
with Tween20 Tris-buffered saline (TBST; $20 \mathrm{mM}$ Tris, $137 \mathrm{mM} \mathrm{NaCl,} 0.1 \%$ (vol/ vol) Tween 20, pH 7.6) containing 3\% (w/v) non-fat dry milk (Campina, Eindhoven, the Netherlands), washed, and incubated overnight at $4{ }^{\circ} \mathrm{C}$ with a target-specific primary antibody (Supplementary Table 2) diluted 1:1,000-1:10,000 in TBST with $3 \%(\mathrm{w} / \mathrm{v}) \mathrm{BSA}$ or non-fat dry milk at $4{ }^{\circ} \mathrm{C}$. Subsequently, membranes were washed and incubated with a HRP-conjugated secondary antibody (\#BA-9200, \#BA-1000, Vector Laboratories, Amsterdam, the Netherlands), diluted 1:10,000 in 3\% (w/v) non-fat dry milk in TBST for $1 \mathrm{~h}$ at room temperature. Thereafter, membranes were washed, incubated for 5 min with $0.5 x$ SuperSignal West PICO or $0.25 x$ West Femto Chemiluminescent Substrate (Thermo Scientific, Landsmeer, the Netherlands), and imaged using the Amersham ${ }^{\mathrm{TM}}$ Imager 600. Original unaltered images were quantified with Image Quant software (GE Healthcare, Eindhoven, the Netherlands). Measured protein quantity was corrected for total protein content. Images included in the figures of this manuscript have been adjusted for brightness and contrast equally throughout the picture.

\section{Statistical analysis}

Data is depicted as bar graphs indicating the mean and SEM as fold change compared to the control. For each comparison, the D'agrostino and Pearson omnibus normality test was used to test normality and subsequently either an unpaired t-test or Mann Whitney test was used accordingly (GraphPad Software, La Jolla California USA). A p-value $<0.05$ was considered as significantly different from the control group and was presented as follows: Ns: $p>0.05,{ }^{*} \mathrm{p} \leq 0.05,{ }^{* *} \mathrm{p} \leq 0.01,{ }^{* * *} \mathrm{p} \leq 0.001$. 


\section{Results}

\section{Increased oxidative stress in PE placentae}

To determine the impact of PE on known ROS-generating processes and on cellular antioxidant defence systems, mRNA abundance and enzymatic activity of key constituents of these pathways were assessed. As shown in Figure 1, TEAC as well as the GSSG/GSH ratio were significantly higher in PE placentae compared to controls (Figure 1A-B). In addition, in PE placentae, transcript levels of catalase 1 (CAT1) were lower while mRNA expression levels of SOD1 and mitochondrial manganese-dependent superoxide dismutase 2 (MnSOD2) were respectively unchanged and significantly higher compared to controls (Figure 1C). No differences were observed for transcript abundance of xanthine oxidase (XO), NADPH oxidase 2 (NOX2) or NOX4 (Supplementary Figure 1A). In addition to these indicators of oxidative stress in PE placentae, both the expression of tumor necrosis factor- $\alpha$ $(\mathrm{TNF}-\alpha)$ as well as the ratio of pro-apoptotic Bcl-2-associated X protein / anti-apoptotic B-cell lymphoma 2 (BAX/BCL-2) mRNA expression levels were significantly higher in PE placentae compared to controls (Supplementary Figure 1B-C).
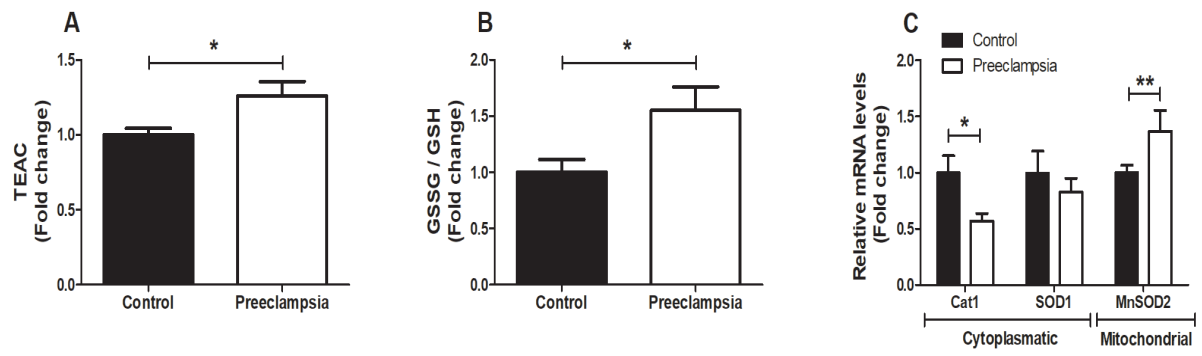

Figure 1. Oxidative stress in PE placentae. TEAC (A) and GSSG/GSH ratio (B) as well as transcript levels of CAT1 and SOD1 and MnSOD2 (C) were assessed in PE as well as control placentae. Data is presented as fold change compared to the control placentae and as mean with SEM from $n=9$ (controls), $\mathrm{n}=8$ (preeclampsia) for assessment of TEAC and GSSG/GSH ratio and $\mathrm{n}=11$ (controls), $\mathrm{n}=12$ (preeclampsia) for assessment of mRNA levels. ${ }^{*} \mathrm{p} \leq 0.05,{ }^{*} \mathrm{p} \leq 0.01$. TEAC: Trolox equivalent antioxidant capacity, GSSG: oxidized glutathione, GSH: Reduced glutathione, Cat1: Catalase-1, SOD1: Superoxide dismutase 1 and MnSOD2: Manganese-dependent superoxide dismutase. 


\section{Lower mitochondrial content and higher activity and abundance of key constituents of glycolysis in placentae of PE patients com- pared to controls}

As mitochondria are known drivers of apoptosis and inflammation (23), and are a well-known source of ROS, and ROS are known to be able to damage mitochondria and impair mitochondrial function (23), we next investigated whether PE affected placental mitochondrial content and mitochondrial (and non-mitochondrial) metabolic processes. Mitochondrial content was significantly lower in PE placentae compared to controls, which was evidenced by a lower mitochondrial DNA (mtDNA) copy number (Figure 2A) and lower total enzyme activity of CS (Figure 2B), two well-described indicators of mitochondrial content (24). No changes were observed in mRNA transcript levels of CS (Supplementary Figure 2A). To investigate if the lower mitochondrial content that we observed in PE placentae was associated with changes in (mitochondrial- and non-mitochondrial) metabolic processes, we next assessed the abundance and activity of key constituents of the electron transport chain (ETC), fatty acid- $\beta$ oxidation (FAO) and glycolysis. Interestingly, while no differences were found in activity or mRNA expression levels of the rate-limiting enzyme of the FAO pathway (Supplementary Figure 2B-C) or in transcript and protein abundance of nuclear-encoded sub-units of ETC complexes (Supplementary Figure 2D-E), we did observe that mRNA transcript levels of the mitochondrial-encoded sub-unit COXII of complex IV of the ETC were significantly lower in PE placentae compared to controls (Figure 2C). Moreover, the PFK activity as well as protein and mRNA abundance of hexokinase II (HKII), two key enzymes involved in glycolysis, were 2-3 fold higher in PE placentae compared to controls (Figure 3A-C). This was also associated with higher transcript levels of the glucose transporter 1 (GLUT-1) in PE placentae (Figure 3D).
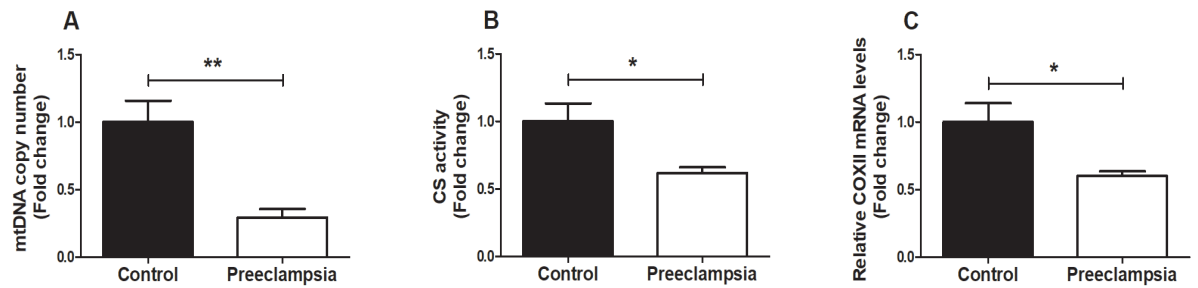

Figure 2. Lower mitochondrial content in PE placentae. Mitochondrial DNA copy number (A), Citrate synthase activity (B) and mRNA transcript levels of COXII (C) were assessed in PE as well as control placentae. Data is presented as fold change compared to the control placentae and as mean with SEM from $n=11$ (controls), $n=12$ (preeclampsia) for assessment of mRNA levels and $n=9$ (controls), $\mathrm{n}=8$ (preeclampsia) for the assessment of CS activity and mtDNA copy number. ${ }^{*} \mathrm{p} \leq 0.05$ and $* * \mathrm{p} \leq$ 0.01. mtDNA: Mitochondrial DNA, CS: Citrate synthase and COXII: Cytochrome c oxidase subunit II. 

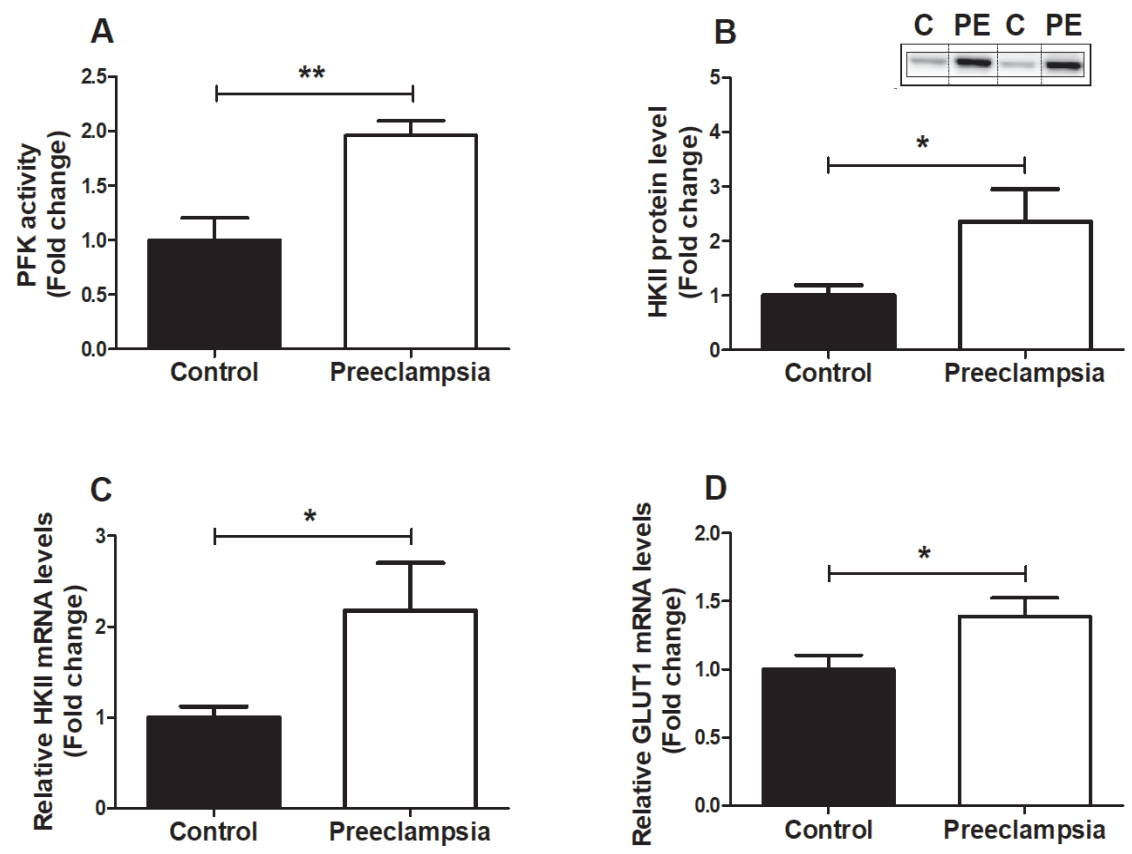

Figure 3. Increased expression and activity of key glycolytic enzymes in PE placentae. PFK enzyme activity (A), HKII protein level (B), mRNA expression levels of HKII (C) and Glut-1 (D) were assessed in PE as well as control placentae. Representative immunoblots are shown and Western blots were corrected for total protein loading assessed by Ponceau S Staining with adjusted contrast equally applied to the whole photograph. Black boxes around the representative pictures indicate that they were cut from the same Western blot. Data is presented as fold change compared to the control placentae and as mean with SEM from $n=11$ (controls), $n=12$ (preeclampsia) for assessment of $m R N A$ levels and $n=$ 9 (C: controls), $n=8$ (PE: preeclampsia) for assessment of the PFK activity and HKII protein levels. Ns: $\mathrm{p}>0.05,{ }^{*} \mathrm{p} \leq 0.05,{ }^{*} \mathrm{p} \leq 0.01$. PFK: Phosphofructokinase, HKII: Hexokinase and GLUT-1: Glucose transporter 1 .

\section{Alterations in the molecular regulation of mitochondrial biogen- esis in PE placentae}

In light of the reductions in mitochondrial content and indications for a metabolic shift away from oxidative metabolism in PE placentae, we next explored whether PE impacts the peroxisome proliferator-activated receptor gamma coactivator 1 (PGC1) signaling network, a key regulatory network controlling mitochondrial biogenesis and mitochondrial oxidative substrate metabolism through the coordinated action of a variety of transcription factors and co-activator molecules (25). As depicted in Figure 4, while protein levels of PGC-1 $\alpha$ were significantly lower in PE placentae, no changes in PGC-1 $\alpha$ transcript levels were observed compared to controls. Placental 
mRNA levels of PGC-1 $\beta$, however were significantly lower in PE. With regard to transcription factors specifically controlling mitochondrial biogenesis, protein levels of nuclear respiratory factor 1 (NRF1) were higher and mRNA expression of NRF1 was lower in PE placentae vs controls. Placental NRF2 $\alpha$ mRNA levels on the other hand were not affected by PE. Additionally, although mitochondrial transcription factor A (Tfam) transcript abundance was lower in PE, no significant differences in Tfam protein levels were observed between both groups. Furthermore, estrogen-related receptor alpha $(\mathrm{ERR} \alpha)$ protein levels were higher in PE while its mRNA levels were lower. Transcript abundance of both peroxisome proliferator-activated receptor (PPAR)- $\alpha$ and PPAR- $\delta$ showed no significant differences between both groups (Figure 4A-B). Collectively, these data indicate significant alterations in the molecular regulation of mitochondrial biogenesis and oxidative substrate metabolism in PE compared to controls.
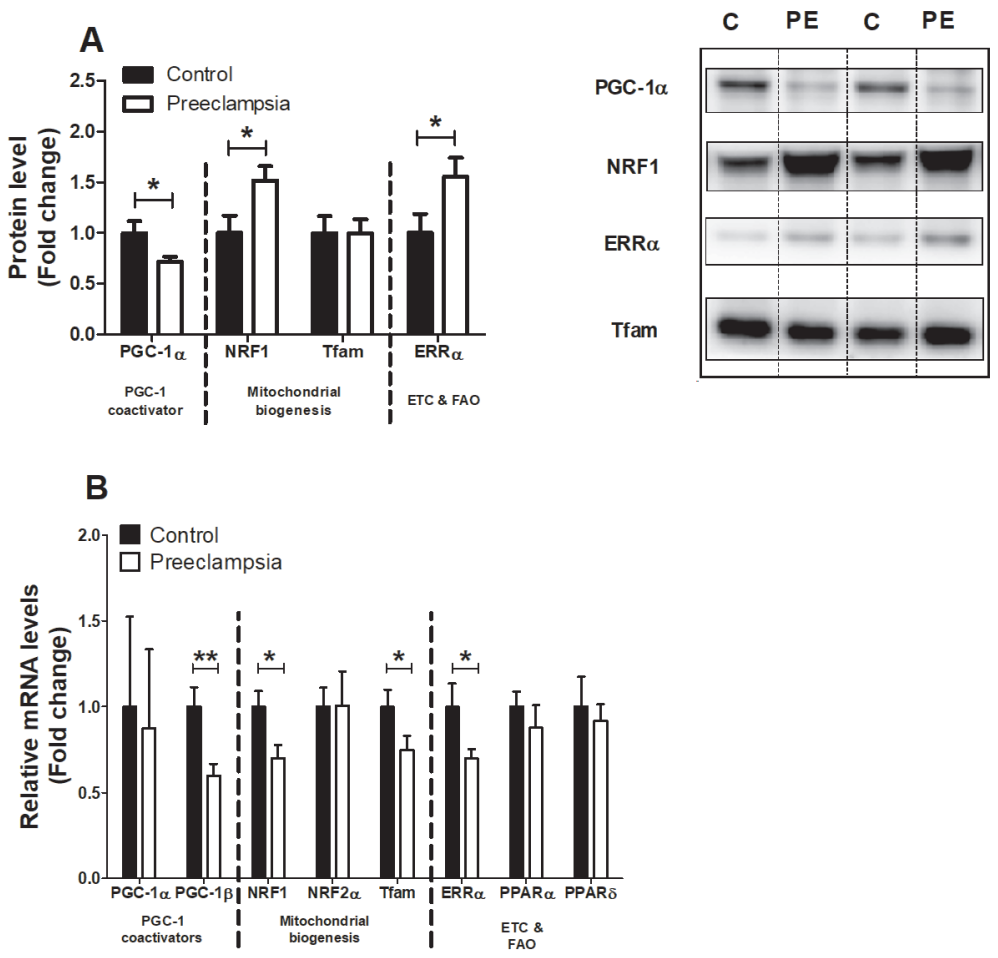

Figure 4. Alterations in the molecular regulation of mitochondrial biogenesis in PE placentae. Protein levels of PGC-1 $\alpha$, NRF1, Tfam and ERR $\alpha$, (A) and mRNA transcript levels of PGC- $1 \alpha$, PGC$1 \beta, \mathrm{NRF} 1, \mathrm{NRF} 2 \alpha$, Tfam, ERR $\alpha$, PPAR $\alpha$ and PPAR $\delta$ (B) were assessed in PE as well as control placentae. Representative immunoblots are shown and Western blots were corrected for total protein loading assessed by Ponceau S Staining with adjusted contrast equally applied to the whole photograph. Black boxes around the representative pictures indicate that they were cut from the same Western blot. Data 
is presented as fold change compared to the control placentae and as mean with SEM from $\mathrm{n}=11$ (controls), $\mathrm{n}=12$ (preeclampsia). ${ }^{*} \mathrm{p} \leq 0.05,{ }^{* *} \mathrm{p} \leq 0.01$. PGC- $1 \alpha$ : Peroxisome proliferator-activated receptor gamma coactivator 1-alpha, NRF1: Nuclear respiratory factor 1, Tfam: Mitochondrial transcription factor A, ERR $\alpha$ : Estrogen-related receptor alpha, PGC-1 $\beta$ : Peroxisome proliferator-activated receptor gamma coactivator 1-beta, NRF2 $\alpha$ : Nuclear respiratory factor 2 alpha, PPAR $\alpha$ : Peroxisome proliferator-activated receptor alpha, PPAR $\delta$ : Peroxisome proliferator-activated receptor delta. ETC: Electron transport chain and FAO: Fatty acid $\beta$-oxidation.

\section{Expression of key constituents of the mitophagy machinery are altered in PE placentae}

As mitochondrial content is not only controlled by the biogenesis of new organelles but is also influenced by breakdown of mitochondria through mitophagy (e.g. mitochondrial-specific autophagy), we next assessed the impact of PE on key constituents of the mitophagy machinery. Moreover, as mitophagy requires several general autophagy-related proteins for generating the autophagosomal membrane and priming the autophagosome to the mitochondria, these proteins were studied as well. As depicted in Figure 5A, in PE placentae, protein levels of BCL2/adenovirus E1B $19 \mathrm{kDa}$ protein-interacting protein 3 (BNIP3) and BCL2/adenovirus E1B $19 \mathrm{kDa}$ protein-interacting protein 3-like (BNIP3L) were significantly higher while abundance of FUN14 domain containing 1 (FUNDC1), the PTEN-induced kinase 1 (PINK1) and E3 ubiquitin-protein ligase Parkin (PARK2) proteins were similar compared to controls. mRNA expression levels of these mitophagy-associated proteins were unaltered in PE placentae compared to the controls with the exception of FUNDC1 transcript levels, which were lower in PE (Figure 5B). In addition, protein and mRNA transcript levels of all general autophagy-associated proteins investigated were not significantly different in PE vs controls. Sequestosome 1 (SQSTM1) protein levels, however, were significantly higher and mRNA transcript levels of optineurin (OPTN) were significantly lower in PE placentae compared to controls (Figure 5C-D). Collectively, these data indicate that proteins specifically involved in receptor-mediated mitophagy (BNIP3, BNIP3L), rather than ubiquitin-mediated mitophagy (PINK1, PARK2), are higher in PE placentae compared to controls. 

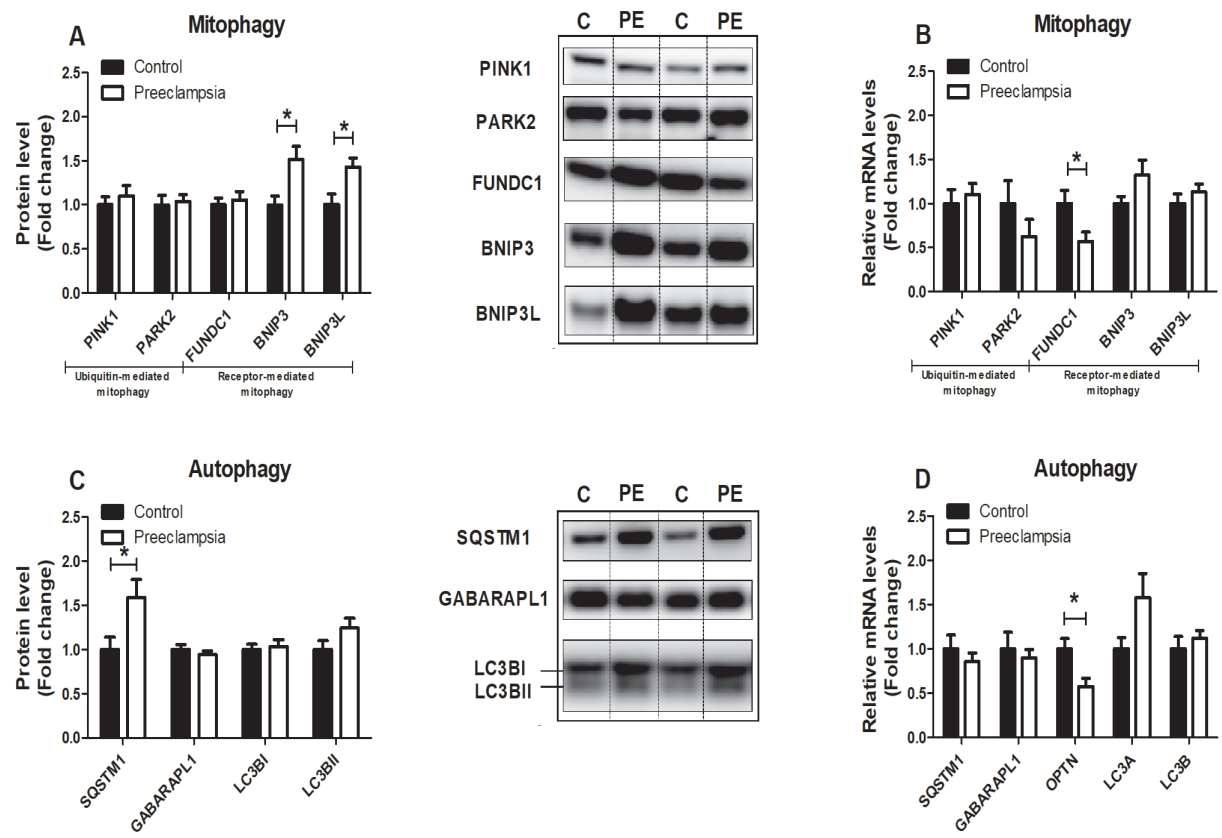

Figure 5. Expression of key constituents of the mitophagy machinery are altered in PE placentae. Mitophagy-associated protein levels of PINK1, PARK2, FUNDC1, BNIP3 and BNIP3L (A), mitophagy-associated mRNA transcript levels of PINK1, PARK2, FUNDC1, BNIP3, BNIP3L (B), autophagy-associated protein levels of SQSTM1, GABARAPL1, LC3BI and LC3BII (C) and autophagy-associated mRNA transcript levels of SQSTM1, GABARAPL1, OPTN and LC3A/B (D) were assessed in $\mathrm{PE}$ as well as control placentae. Representative immunoblots are shown and Western blots were corrected for total protein loading assessed by Ponceau S Staining with adjusted contrast equally applied to the whole photograph. Black boxes around the representative pictures indicate that they were cut from the same Western blot. Data is presented as fold change compared to the control placentae and as mean with SEM from $\mathrm{n}=11$ (controls), $\mathrm{n}=12$ (preeclampsia). ${ }^{*} \mathrm{p} \leq 0.05$. PINK1: PTEN-induced kinase 1, PARK2: E3 ubiquitin-protein ligase Parkin, FUNDC1: FUN14 domain containing 1, BNIP3: BCL2/adenovirus E1B $19 \mathrm{kDa}$ protein-interacting protein 3, BNIP3L: BCL2/adenovirus E1B $19 \mathrm{kDa}$ protein-interacting protein 3-like, SQSTM1: Sequestosome 1, GABARAPL1: GABA Type A Receptor Associated Protein Like 1, LC3B: Microtubule-associated protein 1 light chain 3 beta I/II, OPTN: Optineurin and LC3A: Microtubule-associated protein 1 light chain 3 alpha.

\section{Increased abundance of mitochondrial fission proteins in PE placentae}

As mitochondrial fission and fusion are key events in the processes of mitochondrial biogenesis as well as mitophagy and are essential in maintaining normal mitochondrial homeostasis (26), mRNA and protein levels of several mitochondrial fusion and fission proteins were investigated. Protein levels of dynamin-1-like protein (DNM1L), a key protein involved in mitochondrial fission, was significantly higher in PE 
placentae (Supplementary Figure 3A). Furthermore, mitochondrial fission-related mRNA transcript levels of DNM1L were also significantly higher in PE while fission 1 (Fis-1) transcript levels were unaltered compared to controls (Supplementary Figure 3B). With regard to mediators of mitochondrial fusion, mRNA transcript levels of mitofusin-1 and 2 (Mfn1 and Mfn2) and mitochondrial Optic atrophy protein 1 (Opa1) were not significantly different in PE placentae compared to controls (Supplementary Figure 3C), indicating that specifically mitochondrial fission constituents are higher in PE placentae compared to controls. 


\section{Discussion}

\section{Principal Findings}

In the current study, we show that PE placentae are not only characterized by the presence of oxidative stress but also by a profound reduction in mitochondrial content and an increased abundance of key glycolytic enzymes suggestive of a greater reliance on glycolytic metabolism. Moreover, we show for the first time that these changes are associated with significant alterations in the molecular pathways governing control over mitochondrial biogenesis and changes in key constituents of the machinery controlling mitophagy and mitochondrial fission events. Taken together, our findings suggest significant alterations at the level of the mitochondrion in placentae from women with PE, which may well contribute to PE pathophysiology.

The presence of oxidative stress and associated changes in several intracellular antioxidant systems is well described in PE (10). For example, PE has been associated with decreased activity levels of the anti-oxidant enzymes catalase and superoxide dismutase, increased lipid peroxidation by-products, reduced GSH production, impaired GSSG to GSH conversion and reduced levels of non-enzymatic antioxidants such as thiols, ascorbic acid, $\alpha$-tocopherol and carotenoids in the placenta (27-29). Collectively, these observations are in line with our data. Also, total antioxidant capacity in our study was markedly higher in PE placentae, suggestive of a compensatory response to increased oxidative stress in PE placentae $(27,28)$. Interestingly, we observed no changes in expression levels of NOX 2/4 and XO isoforms, two well-known sources of intracellular ROS, as well as increased transcript levels of the mitochondrial-located MnSOD2. Considering the signs for increased oxidative stress, elevated mitochondrial-specific antioxidant expression in PE placentae and the notion that the mitochondria are the main intracellular site for cellular $\mathrm{O}_{2}$ consumption, these data suggest that mitochondria likely are a significant source for ROS and oxidative stress in PE.

Increased placental mitochondrial ROS may directly damage mtDNA and disrupt mitochondrial metabolic function and mitochondrial biogenesis (30). In this context, we found evidence for significant reductions in mitochondrial content in PE placentae compared to controls, which was illustrated by reductions in mtDNA copy number and reduced citrate synthase activity, both well-established markers of mitochondrial content (24). In the placenta, disorders related to impaired perfusion including IUGR and PE have recently been shown to be associated with changes in mitochondrial content (31). In addition, a recent study using transmission electron microscopy revealed swelling, increased mitochondrial lumen and irregular ar- 
rangement of mitochondrial cristae in early-onset PE placentae (29). The majority of studies investigating mitochondrial content in PE-complicated placentae used whole placental tissue homogenates. However, mitochondria within different cell lineages in the placentae often have distinct functions, different antioxidant capacity and do respond differently to environmental stimuli (31). Indeed, Mando et al. found increased mitochondrial content in whole placental tissue but decreased content in cytotrophoblast cells, which are in direct contact with maternal blood and therefore expected to be most affected by impaired placenta perfusion in PE (32). Furthermore, PE placentae show increased numbers of mitochondria in cytotrophoblast cells, but with reduced size $(33,34)$. which is suggestive for increased mitochondrial fission. In light of these findings, it remains to be established in which cell types of the placenta mitochondrial content is affected.

Besides changes in mitochondrial content, several human studies showed a significant reduction of adenosine triphosphate (ATP) levels in PE placentae (35-37), indicating impaired functioning of the mitochondrial metabolic pathways including the ETC. In line with this, a PE rat model of reduced uterine perfusion pressure, showed lower complex I and complex IV activity in the placenta (38). Our PE placentae showed signs for a decrease in the abundance of complex III of the mitochondrial ETC. This is in line with previous work, which showed reductions in the abundance of COXII and impaired electron flow through complex IV, which these authors suggested to be contributing to excessive mitochondrial ROS formation (39, $40)$. In contrast to previous studies $(35,41,42)$, which in general found a decrease in the expression and activity of metabolic enzymes involved in the initial step of the $\beta$-oxidation pathway, no difference was found in the activity of HADH in our PE placentae compared to the controls. Collectively, our data, in concert with available literature, suggest abnormalities in mitochondrial content and function in PE.

In line with this notion, we show for the first time that mitochondrial abnormalities in PE placentae were associated with increases in the abundance and activity of key glycolytic enzymes, indicating an increased relative contribution of glycolysis in PE. In accordance with these results, a significant increase in the glycolytic intermediate 2-phosphoenolpyruvate was found in severe PE-complicated placentae in humans (35). Furthermore, increased glycolysis upon hypoxic stress, a well know stress factor in PE, has been observed in placental trophoblast cells (43).

In line with the decreased mitochondrial content found in our PE placentae, we observed decreased mRNA and protein levels of PGC-1 co-activator molecules, which are master regulators of mitochondrial biogenesis. Moreover, mRNA expression of 
NRF1 and Tfam, both known to be down-stream of PGC-1 in the induction of mitochondrial biogenesis, was found to be decreased suggesting diminution of mitochondrial biogenesis in PE placentae. Although available evidence is scarce, other studies are in line with these results and showed that in PE placentae, where mitochondrial content was decreased, mRNA expression levels of PGC1- $\alpha$ and NRF1 were also decreased $(44,45)$. Moreover, placental tissue from a reduced uterine perfusion rat model also showed reductions in PGC-1 $\alpha$ protein levels (46), suggesting that impaired placental perfusion may well be linked to mitochondrial adaptations in PE. However, in our study NRF1 and ERR $\alpha$ protein levels were significantly higher in PE placentae compared to controls suggesting a potential compensatory cellular response to increased mitochondrial biogenesis. In this regard, although its regulatory pathways are clearly impacted, it remains to be established whether mitochondrial biogenesis is down- or upregulated in PE.

In addition to mitochondrial biogenesis, selective autophagy of mitochondria (i.e. mitophagy) contributes to the regulation of cellular mitochondrial content. We observed higher levels of the receptor-mediated mitophagy-related proteins BNIP3 and BNIP3L as well as the general autophagy-related protein SQSTM1 in PE placentae compared to controls. Interestingly, these proteins have been shown to be upregulated upon hypoxic stress in the placenta in previous studies $(47,48)$. In contrast, abundance of constituents of the Pink/Park pathway, another key regulatory pathway controlling (ubiquitin-mediated) mitophagy, were not affected by PE in our study indicating that specific mitophagy pathways may be involved in the pathogenesis of PE.

Both mitochondrial biogenesis and mitophagy require mitochondrial fusion and fission events. ROS production regulates mitochondrial fission and fusion in healthy cells, providing a mechanism that regulates mitochondrial morphology and function, which is dependent on the redox state (49). Both mitochondrial fission and fusion are important mechanisms in the maintenance of mitochondrial health, and changes in these processes could therefore have a large impact on mitochondrial function (50). In this context, we now show increased fission-related DNM1L protein and mRNA transcript levels in placentae complicated with PE, while the expression of mitochondrial fusion genes was unaltered. Therefore, the balance between mitochondrial fusion and fission may be tilted towards increased mitochondrial fission in PE, which can contribute to aberrant mitochondrial morphology and function in PE-complicated placentae. 
Collectively, our data show extensive abnormalities at the level of the mitochondrion and the regulatory pathways controlling mitochondrial content and function in PE placentae. This suggests a large-scale mitochondrial dysfunction in PE placentae, which may well contribute to the pathogenesis of PE. Indeed, dysfunctional mitochondria are not only closely linked to excessive ROS formation, but also to inflammation as well as apoptosis, both of which have been essentially implicated in the pathogenesis of PE $(33,51,52)$. Indeed, levels of many apoptotic proteins as well as in intracellular $\mathrm{Ca}^{2+}$ are altered in $\mathrm{PE}$ placentae $(53,54)$. In agreement with this data, we found increased mRNA transcript levels of the BAX/BCL-2 ratio in our PE placentae. Moreover, it has been shown that increased expression levels of BNIP3, as observed in our study, led to a decreased mitochondrial membrane potential, and triggers cells apoptosis or autophagy by the activation of the Bax/Bak or LC-3/Beclin 1 signaling pathway (29). In addition to a prominent role in the control of apoptosis, earlier studies found activation of the NF- $\mathrm{KB}$ pathway and increased abundance of pro-inflammatory cytokines in placentae and syncytiotrophoblast, respectively, in women with early PE $(1,55)$, which is in line with the increased pro-inflammatory mRNA transcript levels of TNF- $\alpha$ found in our PE placentae.

\section{Clinical Implications}

$\mathrm{PE}$ is generally associated with placental oxidative stress, which is believed to play a key role in the pathophysiology of the disease. The underlying molecular mechanisms for the excessive production of placental ROS, however, remain unclear (10). Considering the signs for increased oxidative stress and mitochondrial dysfunction found in our PE placenta, and the notion that the mitochondria are the main intracellular site for cellular $\mathrm{O}_{2}$ consumption, it is feasible that mitochondria are a significant source for the increased production of placental ROS in PE. This may open up new avenues for new treatment options of PE. Illustrative of the clinical relevance of our findings, in a rodent model for PE (chronic placental hypoxia) administration of the mitochondrially targeted antioxidant MitoQ, improved foetal outcomes, including birth weight and developmental programming of cardiovascular diseases (17-20). Whether or not this therapeutic strategy has potential for the treatment of PE in humans, remains to be established. As observed in our study, increased placental mitochondrial ROS may directly damage mtDNA, inhibit mitochondrial biogenesis and promote mitophagy. The increased reliance on glycolysis in PE placentae may be a consequence of the decreased mitochondrial content and impaired functioning of the ETC. This shift to anaerobic respiration may be a protective response of the placenta to the impaired placental perfusion in $\mathrm{PE}$ and so sparing $\mathrm{O}_{2}$ for the growing foetus. Our study now provides valuable new insights into the pathophysiology of $\mathrm{PE}$ and more specifically, into how specific mitochondrial abnormalities in PE pla- 
centae may well be linked to the development of oxidative stress, inflammation and apoptosis thereby adding to our understanding how mitochondrially targeted therapeutics may be beneficial for the treatment of PE.

\section{Research Implications}

This study is the first to comprehensively characterize and assess mitochondrial content and the regulatory pathways controlling mitochondrial content and function in PE placentas. Moreover, our study may help to explain why despite the strong association of PE with oxidative stress, human studies using systematically acting antioxidants as a treatment for PE are generally unsuccessful (15), and mitochondrially targeted antioxidants my serve as a better therapeutic target. In addition, our study provides evidence that suggests that abnormalities at the level of the mitochondrion may well be central to key aspects of PE pathophysiology as it is currently well-known that besides their traditional role in energy production, mitochondria are known regulators of apoptosis, oxidative stress and inflammation, all of which have been heavily implicated in PE pathophysiology. Collectively, this opens up a new avenue of research into the contribution of mitochondrial dysfunction to PE pathophysiology. More research is necessary to unravel which PE-associated factors (e.g. hypoxia) trigger mitochondrial abnormalities in the placenta, in which cell types this is apparent and to what extent this contributes to PE pathophysiology. In addition, whether or not mitochondrially targeted strategies have therapeutic potential in the treatment of PE remains to be discovered in more detail.

\section{Strengths and Limitations}

Our study is the first one that provides a detailed overview of mitochondrial abnormalities and the pathways controlling mitochondrial function and content in $\mathrm{PE}$, which is considered a strength of our study. One obvious limitation, however, is that in our study, we used whole placental tissue homogenates so it remains to be established in which cell types of the placenta mitochondrial content is affected. Furthermore, a well-recognized difficulty is the lack of non-laboured, healthy preterm control placental samples, as caesarean sections are rarely performed in obstetrically normal pregnancies at gestational age equivalent to those in early onset pre-eclampsia. Despite the mean gestational age in the PE group of 33 weeks, the group contains also cases of late onset PE. Due to the relative small group and the variation of the parameters tested in this study, it was not possible to divide the group into two sub groups (early and late onset PE). Based on the findings of Holland et al (1), the alterations in mitochondria found in our study may even be an underestimation of the presence of mitochondrial abnormalities in early onset PE placentas. In addition, as mitophagy is a flux, changes in protein and mRNA expression levels 
of these molecules that we assessed in our study are not per se indicative of active or inhibited mitophagy but rather are to be taken as indications of potential changes in the process of mitophagy. The same concept holds true for the process of mitochondrial biogenesis. Moreover, we did not directly assess mitochondrial function by means of respirometry, which prevents hard conclusions regarding (changes in) mitochondrial functionality in PE. Therefore, future studies including measurements of mitochondrial respiration, and assessment of actual mitophagy and mitochondrial biogenesis including electron microscopy and recently developed assays for measuring mitophagy (56), e.g. MitoTimer, mt-Keima, and Mito-QC, would provide beneficial knowledge.

\section{Conclusions}

In conclusion, this study demonstrates that PE is associated with significant abnormalities at the level of the mitochondrion in the placenta, and that these abnormalities may well be linked to the development of oxidative stress. This implies that mitochondrially targeted antioxidant-based intervention aimed at preventing mitochondrial dysfunction and excessive ROS formation may have therapeutic potential in pregnancy complications like PE.

\section{Acknowledgements}

This study was supported by NUTRIM Graduate Program. There was no additional external funding received for this study. 


\section{References}

1. Holland OJ, Cuffe JSM, Dekker Nitert M, Callaway L, Kwan Cheung KA, Radenkovic F, et al. Placental mitochondrial adaptations in preeclampsia associated with progression to term delivery. Cell Death Dis. 2018;9(12):1150.

2. Vangrieken P, Al-Nasiry S, Janssen GMJ, Weseler AR, Spaanderman ME, Bast A, et al. The direct and sustained consequences of severe placental hypoxia on vascular contractility. PLoS One. 2018;13(8):e0202648.

3. Phipps E, Prasanna D, Brima W, Jim B. Preeclampsia: updates in pathogenesis, definitions, and guidelines. Clinical Journal of the American Society of Nephrology. 2016;11(6):1102-13.

4. Liu L, Oza S, Hogan D, Chu Y, Perin J, Zhu J, et al. Global, regional, and national causes of under-5 mortality in 2000-15: an updated systematic analysis with implications for the Sustainable Development Goals. Lancet. 2016;388(10063):302735 .

5. Wu CS, Nohr EA, Bech BH, Vestergaard M, Catov JM, Olsen J. Health of children born to mothers who had preeclampsia: a population-based cohort study. American journal of obstetrics and gynecology. 2009;201(3):269. e1-. e10.

6. Redman CW, Sargent IL. Placental debris, oxidative stress and pre-eclampsia. Placenta. 2000;21(7):597-602.

7. Hung TH, Burton GJ. Hypoxia and reoxygenation: a possible mechanism for placental oxidative stress in preeclampsia. Taiwan J Obstet Gynecol. 2006;45(3):189200 .

8. Robson A, Harris LK, Innes BA, Lash GE, Aljunaidy MM, Aplin JD, et al. Uterine natural killer cells initiate spiral artery remodeling in human pregnancy. The FASEB Journal. 2012;26(12):4876-85.

9. Mailloux RJ. Teaching the fundamentals of electron transfer reactions in mitochondria and the production and detection of reactive oxygen species. Redox Biol. 2015;4:381-98.

10. Chiarello DI, Abad C, Rojas D, Toledo F, Vazquez CM, Mate A, et al. Oxidative stress: Normal pregnancy versus preeclampsia. Biochim Biophys Acta Mol Basis Dis. 2018.

11. Zou Y, Zuo Q, Huang S, Yu X, Jiang Z, Zou S, et al. Resveratrol inhibits trophoblast apoptosis through oxidative stress in preeclampsia-model rats. Molecules. 2014;19(12):20570-9.

12. Afkham A, Eghbal-Fard S, Heydarlou H, Azizi R, Aghebati-Maleki L, Yousefi M. Toll-like receptors signaling network in pre-eclampsia: An updated review. J Cell Physiol. 2019;234(3):2229-40.

13. Heazell AE, Taylor NN, Greenwood SL, Baker PN, Crocker IP. Does altered 
oxygenation or reactive oxygen species alter cell turnover of BeWo choriocarcinoma cells? Reprod Biomed Online. 2009;18(1):111-9.

14. Redman CW, Sargent IL. Placental stress and pre-eclampsia: a revised view. Placenta. 2009;30 Suppl A:S38-42.

15. Salles AM, Galvao TF, Silva MT, Motta LC, Pereira MG. Antioxidants for preventing preeclampsia: a systematic review. ScientificWorldJournal. 2012;2012:243476.

16. Veith C, Boots AW, Idris M, van Schooten F-J, van der Vliet A. Redox Imbalance in Idiopathic Pulmonary Fibrosis: A Role for Oxidant Cross-Talk Between NADPH Oxidase Enzymes and Mitochondria. Antioxidants \& redox signaling. 2019.

17. Nuzzo AM, Camm EJ, Sferruzzi-Perri AN, Ashmore TJ, Yung HW, Cindrova-Davies T, et al. Placental Adaptation to Early-Onset Hypoxic Pregnancy and Mitochondria-Targeted Antioxidant Therapy in a Rodent Model. Am J Pathol. 2018;188(12):2704-16.

18. Tong W, Giussani DA. Preeclampsia link to gestational hypoxia. J Dev Orig Health Dis. 2019:1-12.

19. Aljunaidy MM, Morton JS, Cooke CM, Davidge ST. Prenatal hypoxia and placental oxidative stress: linkages to developmental origins of cardiovascular disease. Am J Physiol Regul Integr Comp Physiol. 2017;313(4):R395-R9.

20. Burton GJ, Yung HW, Murray AJ. Mitochondrial - Endoplasmic reticulum interactions in the trophoblast: Stress and senescence. Placenta. 2017;52:146-55.

21. Shepherd D, Garland PB. The kinetic properties of citrate synthase from rat liver mitochondria. Biochem J. 1969;114(3):597-610.

22. Bergmeyer H, Gawehn K, Grassl M. 3-Hydroxyacyl-CoA dehydrogenase. Methods of enzymatic analysis. 1974;1:474.

23. Wang Y, Nartiss Y, Steipe B, McQuibban GA, Kim PK. ROS-induced mitochondrial depolarization initiates PARK2/PARKIN-dependent mitochondrial degradation by autophagy. Autophagy. 2012;8(10):1462-76.

24. Larsen S, Nielsen J, Hansen CN, Nielsen LB, Wibrand F, Stride N, et al. Biomarkers of mitochondrial content in skeletal muscle of healthy young human subjects. J Physiol. 2012;590(14):3349-60.

25. Theeuwes WF, Gosker HR, Langen RCJ, Verhees KJP, Pansters NAM, Schols A, et al. Inactivation of glycogen synthase kinase-3beta (GSK-3beta) enhances skeletal muscle oxidative metabolism. Biochim Biophys Acta Mol Basis Dis. 2017;1863(12):3075-86.

26. Moreira OC, Estébanez B, Martínez-Florez S, Paz JAd, Cuevas MJ, González-Gallego J. Mitochondrial function and mitophagy in the elderly: effects of exercise. Oxidative medicine and cellular longevity. 2017;2017. 
27. Aydin S, Benian A, Madazli R, Uludag S, Uzun H, Kaya S. Plasma malondialdehyde, superoxide dismutase, sE-selectin, fibronectin, endothelin-1 and nitric oxide levels in women with preeclampsia. Eur J Obstet Gynecol Reprod Biol. 2004;113(1):21-5.

28. Llurba E, Gratacos E, Martin-Gallan P, Cabero L, Dominguez C. A comprehensive study of oxidative stress and antioxidant status in preeclampsia and normal pregnancy. Free Radical Bio Med. 2004;37(4):557-70.

29. Xu Z, Jin X, Cai W, Zhou M, Shao P, Yang Z, et al. Proteomics Analysis Reveals Abnormal Electron Transport and Excessive Oxidative Stress Cause Mitochondrial Dysfunction in Placental Tissues of Early-Onset Preeclampsia. Proteomics Clin Appl. 2018;12(5):e1700165.

30. Hastie R, Lappas M. The effect of pre-existing maternal obesity and diabetes on placental mitochondrial content and electron transport chain activity. Placenta. 2014;35(9):673-83.

31. Holland O, Dekker Nitert M, Gallo LA, Vejzovic M, Fisher JJ, Perkins AV. Review: Placental mitochondrial function and structure in gestational disorders. Placenta. 2017;54:2-9.

32. Scifres CM, Nelson DM. Intrauterine growth restriction, human placental development and trophoblast cell death. J Physiol. 2009;587(Pt 14):3453-8.

33. Ausman J, Abbade J, Ermini L, Farrell A, Tagliaferro A, Post M, et al. Ceramide-induced BOK promotes mitochondrial fission in preeclampsia. Cell Death Dis. 2018;9(3):298.

34. Zsengeller ZK, Rajakumar A, Hunter JT, Salahuddin S, Rana S, Stillman IE, et al. Trophoblast mitochondrial function is impaired in preeclampsia and correlates negatively with the expression of soluble fms-like tyrosine kinase 1. Pregnancy Hypertens. 2016;6(4):313-9.

35. Zhou X, Han TL, Chen H, Baker PN, Qi H, Zhang H. Impaired mitochondrial fusion, autophagy, biogenesis and dysregulated lipid metabolism is associated with preeclampsia. Exp Cell Res. 2017;359(1):195-204.

36. Yu J, Guo X, Chen R, Feng L. Downregulation of Mitofusin 2 in Placenta Is Related to Preeclampsia. Biomed Res Int. 2016;2016:6323086.

37. Padmini E, Lavanya S, Uthra V. Preeclamptic placental stress and over expression of mitochondrial HSP70. Clin Chem Lab Med. 2009;47(9):1073-80.

38. Vaka VR, McMaster KM, Cunningham MW, Jr., Ibrahim T, Hazlewood R, Usry N, et al. Role of Mitochondrial Dysfunction and Reactive Oxygen Species in Mediating Hypertension in the Reduced Uterine Perfusion Pressure Rat Model of Preeclampsia. Hypertension. 2018;72(3):703-11.

39. Wang Z, Zhang G, Lin M. Mitochondrial tRNA(leu)(UUR) gene mutation and the decreased activity of cytochrome c oxidase in preeclampsia. J Tongji Med 
Univ. 1999;19(3):209-11.

40. Muralimanoharan S, Maloyan A, Mele J, Guo C, Myatt LG, Myatt L. MIR210 modulates mitochondrial respiration in placenta with preeclampsia. Placenta. 2012;33(10):816-23.

41. Shi Z, Long W, Zhao C, Guo X, Shen R, Ding H. Comparative proteomics analysis suggests that placental mitochondria are involved in the development of pre-eclampsia. PLoS One. 2013;8(5):e64351.

42. Bartha JL, Visiedo F, Fernandez-Deudero A, Bugatto F, Perdomo G. Decreased mitochondrial fatty acid oxidation in placentas from women with preeclampsia. Placenta. 2012;33(2):132-4.

43. Xie Y, Zhou S, Jiang Z, Dai J, Puscheck EE, Lee I, et al. Hypoxic stress induces, but cannot sustain trophoblast stem cell differentiation to labyrinthine placenta due to mitochondrial insufficiency. Stem cell research. 2014;13(3):478-91.

44. Mando C, De Palma C, Stampalija T, Anelli GM, Figus M, Novielli C, et al. Placental mitochondrial content and function in intrauterine growth restriction and preeclampsia. Am J Physiol Endocrinol Metab. 2014;306(4):E404-13.

45. Poidatz D, Dos Santos E, Duval F, Moindjie H, Serazin V, Vialard F, et al. Involvement of estrogen-related receptor-gamma and mitochondrial content in intrauterine growth restriction and preeclampsia. Fertil Steril. 2015;104(2):483-90.

46. Delany A, McCarthy F, Walsh S, Kenny L. PP053. The role of peroxisome proliferator-activated receptor gamma co-activator 1-alpha in pregnancy. Pregnancy Hypertens. 2013;3(2):86.

47. Knyazev EN, Zakharova GS, Astakhova LA, Tsypina IM, Tonevitsky AG, Sukhikh GT. Metabolic Reprogramming of Trophoblast Cells in Response to Hypoxia. Bull Exp Biol Med. 2019;166(3):321-5.

48. Bellot G, Garcia-Medina R, Gounon P, Chiche J, Roux D, Pouysségur J, et al. Hypoxia-induced autophagy is mediated through hypoxia-inducible factor induction of BNIP3 and BNIP3L via their BH3 domains. Molecular and cellular biology. 2009;29(10):2570-81.

49. Willems PH, Rossignol R, Dieteren CE, Murphy MP, Koopman WJ. Redox Homeostasis and Mitochondrial Dynamics. Cell Metab. 2015;22(2):207-18.

50. Romanello V, Sandri M. Mitochondrial Quality Control and Muscle Mass Maintenance. Front Physiol. 2015;6:422.

51. Lopez-Armada MJ, Riveiro-Naveira RR, Vaamonde-Garcia C, Valcarcel-Ares MN. Mitochondrial dysfunction and the inflammatory response. Mitochondrion. 2013;13(2):106-18.

52. McCarthy CM, Kenny LC. Mitochondrial [dys]function; culprit in pre-eclampsia? Clin Sci (Lond). 2016;130(14):1179-84.

53. Cali U, Cavkaytar S, Sirvan L, Danisman N. Placental apoptosis in pre- 
eclampsia, intrauterine growth retardation, and HELLP syndrome: an immunohistochemical study with caspase-3 and bcl-2. Clin Exp Obstet Gynecol. 2013;40(1):458.

54. Hache S, Takser L, LeBellego F, Weiler H, Leduc L, Forest JC, et al. Alteration of calcium homeostasis in primary preeclamptic syncytiotrophoblasts: effect on calcium exchange in placenta. J Cell Mol Med. 2011;15(3):654-67.

55. Redman CW, Staff AC. Preeclampsia, biomarkers, syncytiotrophoblast stress, and placental capacity. Am J Obstet Gynecol. 2015;213(4 Suppl):S9 e1, S911.

56. Williams JA, Zhao K, Jin S, Ding W-X. New methods for monitoring mitochondrial biogenesis and mitophagy in vitro and in vivo. Experimental Biology and Medicine. 2017;242(8):781-7. 


\section{Supplementary information}

\section{Supplementary Figures}

\section{Increased inflammation and apoptosis in PE placentae}

mRNA abundance of key constituents of xanthine oxidase and NADPH oxidases were not significantly different in PE placentae compared to controls (Supplementary Figure 1A). In addition, expression of tumor necrosis factor- $\alpha$ (TNF- $\alpha$ ) was higher in PE placental tissue (Supplementary Figure 1B). As mitochondrial processes are closely linked to the coordination of apoptosis, mRNA transcript levels of pro-apoptotic Bcl-2-associated X protein (BAX) and the anti-apoptotic B-cell lymphoma 2 (BCL2) were measured. An increase in the pro-apoptotic BAX/BCL2 mRNA ratio was observed in PE placentae compared to controls (Supplementary Figure 1C).
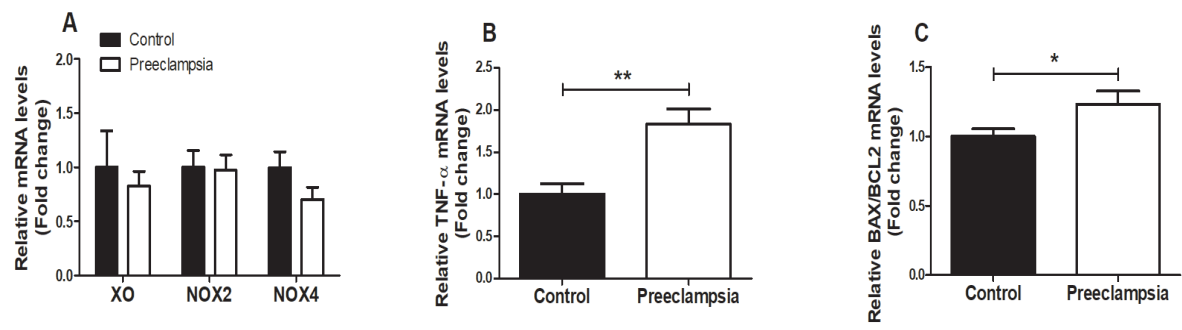

Supplementary Figure 1. Increased inflammation and apoptosis in PE placentae. Transcript levels of XO, NOX2 and NOX4 (A), TNF- $\alpha$ (B) and ratio of BAX/BCL2 transcript levels (C) were assessed in $\mathrm{PE}$ as well as control placentae. Data is presented as fold change compared to the control placentae and as mean with SEM from $\mathrm{n}=11$ (controls), $\mathrm{n}=12$ (preeclampsia). ${ }^{\mathrm{p}} \leq 0.05, * * \mathrm{p} \leq 0.01$. XO: Xanthine oxidase, NOX2: NADPH oxidase 2, NOX4: NADPH oxidase 4, TNF- $\alpha$ : Tumor necrosis factor $\alpha$, BAX: Pro-apoptotic Bcl-2-associated X protein and BCL: Anti-apoptotic B-cell lymphoma 2.

\section{Expression of key constituents of mitochondrial metabolic processes in PE placentae.}

TCA cycle-related mRNA transcript levels of CS, activity and mRNA expression levels of the rate-limiting enzyme of the FAO pathway (HADH) were not significantly different in PE placentae compared to controls (Supplementary Figure 2A-C). Protein as well as mRNA transcript abundance of key constituents of the electron transport chain (nuclear-encoded OXPHOS sub-units) were not different in PE compared to controls (Supplementary Figure 2D-E). 

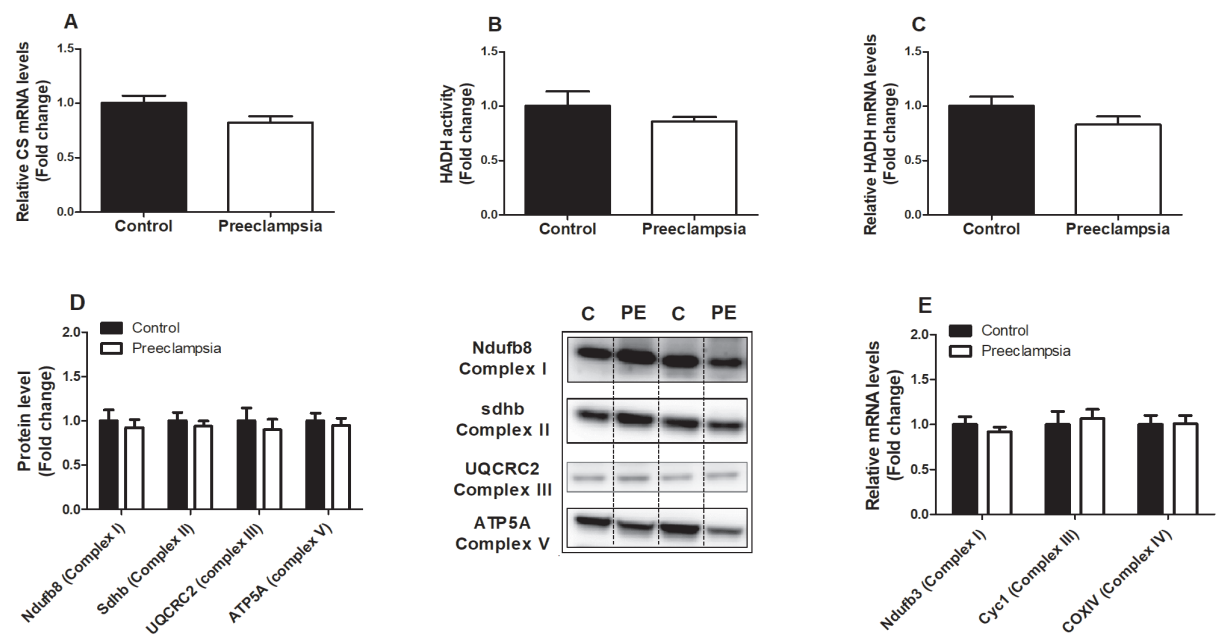

Supplementary Figure 2. Expression of key constituents of mitochondrial metabolic processes in PE placentae. mRNA transcript levels of CS (A), HADH enzyme activity (B), mRNA transcript levels of HADH (C) and protein levels of nuclear-encoded OXPHOS sub-units (Ndufb8, Sdhb, UQCRC2 and ATP5A respectively Complex I, II, III and V) (D) and mRNA transcript levels of nuclear-encoded OXPHOS sub-units (Ndufb, Cycl-1 and COXIV respectively Complex I, III and IV) (E) were assessed in $\mathrm{PE}$ as well as control placentae. Representative immunoblots are shown and Western blots were corrected for total protein loading assessed by Ponceau S Staining with adjusted contrast equally applied to the whole photograph. Black boxes around the representative pictures indicate that they were cut from the same Western blot. Data is presented as fold change compared to the control placentae and as mean with SEM from $n=11$ (controls), $n=12$ (preeclampsia) for the mRNA experiments and $n=9$ (C: controls), $\mathrm{n}=8$ (PE: preeclampsia) for the HADH activity assay. Ns: $\mathrm{p}>0.05,{ }^{*} \mathrm{p} \leq 0.05$. CS: Citrate synthase, HADH: 3-hydroxyacyl-CoA dehydrogenase, Ndufb3: NADH dehydrogenase [ubiquinone] 1 beta subcomplex subunit 3, Sdhb: Succinate dehydrogenase [ubiquinone] iron-sulfur subunit, UQCRC2: Cytochrome b-c1 complex subunit 2, ATP5A: ATP synthase F1 subunit alpha, Cyc1: Cytochrome C1, and COXIV: Cytochrome c oxidase subunit IV.

\section{Increased abundance of mitochondrial fission proteins in PE placentae}

Protein levels of dynamin-1-like protein (DNM1L), a key protein involved in mitochondrial fission, was significantly higher in PE placentae (Supplementary Figure 3A). Furthermore, mitochondrial fission-related mRNA transcript levels of DNM1L were also significantly higher in PE while fission 1 (Fis-1) transcript levels were unaltered compared to controls (Supplementary Figure 3B). With regard to mediators of mitochondrial fusion, mRNA transcript levels of mitofusin-1 and 2 (Mfn1 and Mfn2) and mitochondrial Optic atrophy protein 1 (Opa1) were not significantly different in PE placentae compared to controls (Supplementary Figure 3C), indicating that specifically mitochondrial fission constituents are higher in PE placentae compared to controls. 

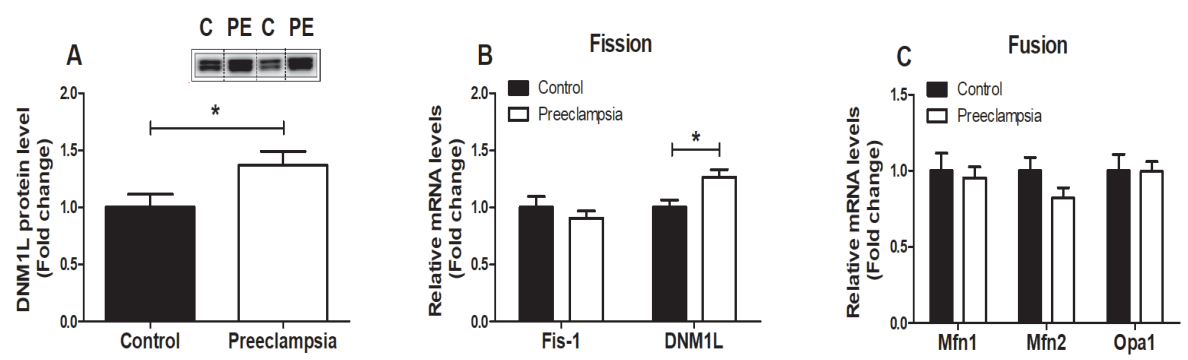

Supplementary Figure3. Increased abundance of mitochondrial fission proteins in PE placentae. Fission-related protein levels of DNM1L (A), fission-related mRNA transcript levels of Fis-1 and DNM1L (B) and fusion-related mRNA transcript levels of Mfn1, Mfn2 and Opa1 (C) were assessed in PE as well as control placentae. Representative immunoblots are shown and Western blots were corrected for total protein loading assessed by Ponceau S Staining with adjusted contrast equally applied to the whole photograph. Black boxes around the representative pictures indicate that they were cut from the same Western blot. Data is presented as fold change compared to the control placentae and as mean with SEM from $n=11$ (controls), $n=12$ (preeclampsia. $* \mathrm{p} \leq 0.05$. DNM1L: Dynamin-1-like protein, Fis-1: Fission 1 protein, Mfn1: Mitofusin-1, Mfn2: Mitofusin-2 and Opa1: Optic atrophy protein 1. 


\section{Supplementary Tables}

\section{Supplementary Table 1. Primers used for qPCR}

\begin{tabular}{|c|c|c|}
\hline Target & Sense primer (5'-3') & Antisense primer (3'-5') \\
\hline Cat1 & GATGTGCATGCAGGACAATCAG & GCTTCTCAGCATTGTACTTGTCC \\
\hline$x O$ & AGAAAGTTGGGGCTGAGTGG & GCAGGCATTGGCAGAAAAGT \\
\hline NOX2 & GACGGCCCAACTGGGATAAT & AGGGTTTCAGCCAAGGCTTC \\
\hline NOX4 & GTAGGAGACTGGACAGAACGA & ATGAAGGGCAGAATTTCGGAGT \\
\hline SOD1 & GGTCCTCACTTTAATCCTCTAT & CATCTTTGTCAGCAGTCACATT \\
\hline MnSOD2 & TGGACAAACCTCAGCCCTAACG & TGATGGCTTCCAGCAACTCCC \\
\hline$T N F-\alpha$ & ATGCCTTTAGATGTGAGCTAACAGTAGGTA & CGTACAGCCATCAAAAAGGGACAC \\
\hline$B A X$ & GGTCTTTTTCCGAGTGGCAG & CACAGGGCCTTGAGCAC \\
\hline$B C L-2$ & GTCTTTTTCCGAGTGGCAGC & GTAGAAAAGGGCGACAACCC \\
\hline RPL13A & CCTGGAGGAGAAGAGGAAAGAGA & $\begin{array}{l}\text { TTGAGGACCTCTGTGTATTTGTCAA } \\
\end{array}$ \\
\hline COXII & ACCTGCGACTCCTTGACGTT & GGGGGCTTCAATCGGGAGTA \\
\hline CS & GATGTGTCAGATGAGAAGTTACGAGACT & $\begin{array}{l}\text { TGGCCATAGCCTGGAACAA } \\
\end{array}$ \\
\hline HADH & TGGCTTCCCGCCTTGTC & TGGAGCCGGTCCACTATCTTC \\
\hline HKII & GTAAATACAGTGGATCTCAATCTTCGGG & CAAGGATTTGAGATGATTCGCTATTCA \\
\hline GLUT1 & TCTGGGCTGCCGGGTTCTAG & $\begin{array}{l}\text { TTTGCAGGCTCCCACAGGC } \\
\end{array}$ \\
\hline Cycl1 & GCATTCGGAGGGGTTTCCAG & CCGCATGAACATCTCCCCA \\
\hline COXIV & CCATGGATGAGAAAGTCGAGT & CGTTCGAGCCCCTGTTCA \\
\hline$P G C-1 \alpha$ & AAGCCACTACAGACACCGC & TCGTAGCTGTCATACCTGGG \\
\hline$P G C-1 \beta$ & CAGAACAAGGAGGCGGAGGTC & AGGTCCAAGTTTGCGAAGC \\
\hline NRF1 & GCACCTTTGGAGAATGTGGT & CTGGGATAAATGCCCGAAG \\
\hline NRF2 & CTCACCTGGGAACAGAACAGGAA & $\begin{array}{l}\text { ACCCAAGAAATGCAGTCTCGAGC } \\
\end{array}$ \\
\hline ERRa & TGCTGCTCACGCTACCGCTC & TCGAGCATCTCCAAGAACAGC \\
\hline Ifam & GAAAGATTCCAAGAAGCTAAGGGTGATT & TCCAGTTTTCCTTTACAGTCTTCAGCTTTT \\
\hline PPARa & CAGAACAAGGAGGCGGAGGTC & AGGTCCAAGTTTGCGAAGC \\
\hline PPAR & TGACCAAAAAGAAGGCCCGC & GTCGTGGATCACAAAGGGCG \\
\hline SQSTM1 & GGTGCACCCCAATGTGATCT & CGCAGACGCTACACAAGTCG \\
\hline PINK1 & GAAAGCCGCAGCTACCAAGA & AGCACATTTGCGGCTACTCG \\
\hline PARK2 & GGTTTGCCTTCTGCCGGGAATG & CTTTCATCGACTCTGTAGGCCTG \\
\hline FUNDC1 & GAAACGAGCGAACAAAGCAG & GCAAAAAGCCTCCCACAAAT \\
\hline BNIP3 & AGCGCCCGGGATGCA & CCCGTTCCCATTATTGCTGAA \\
\hline BNIP3L & CTGCGAGGAAAATGAGCAGTCTCT & $\begin{array}{l}\text { GCCCCCCATTTTTCCCATTG } \\
\end{array}$ \\
\hline OPTN & AAGGAGCAACTGGCATTGCA & $\begin{array}{l}\text { TCTCCATCAAGGACTGCCTG } \\
\end{array}$ \\
\hline GABARAPL1 & ATCGGAAAAAGGAAGGAGAAAAGATC & CAGGCACCCTGGCTTTTGG \\
\hline$L C 3 A$ & CCTGGACAAGACCAAGTTTTTG & GTCTTTCTCCTGCTCGTAGATG \\
\hline$L C 3 B$ & ACCATGCCGTCGGAGAAGAC & TCTCGAATAAGTCGGACATCTTCTACTCT \\
\hline FIS-1 & CCTGGTGCGGAGCAAGTACAA & TCCTTGCTCCCTTTGGGCAG \\
\hline DNMIL & CGACTCATTAAATCATATTTTCTCATTGTCAG & TGCATTACTGCCTTTGGCACACT \\
\hline Mfn 1 & CTGAGGATGATTGTTAGCTCCACG & CAGGCGAGCAAAAGTGGTAGC \\
\hline Mfn2 & TGGACCACCAAGGCCAAGGA & TCTCGCTGGCATGCTCCAC \\
\hline opa1 & TACCAAAGGCATTTTGTAGATTCTGAGTT & GCATGCGCTGTATACGCCAA \\
\hline Ndufb3 & ACAGACAGTGGAAAATTGAAGGG & GCCCATGTATCTCCAAGCCT \\
\hline
\end{tabular}

Cat1: Catalase-1, XO: Xanthine oxidase, NOX2: NADPH oxidase 2, NOX4: NADPH oxidase 4, SOD1: Superoxide dismutase 1, MnSOD2: Manganese-dependent superoxide dismutase, TNF- $\alpha$ : Tumornecrosefactor $\alpha$, BAX: Pro-apoptotic Bcl-2-associated X protein, BCL: Anti-apoptotic B-cell lymphoma 2, RPL13A: Ribosomal Protein L13a, COXII: Cytochrome c oxidase subunit II, CS: Citrate synthase, HADH: 3-hydroxyacyl-CoA dehydrogenase, HKII: Hexokinase, GLUT1: Glucose transporter 1, Cyc1: Cytochrome C1, COXIV: Cytochrome c oxidase subunit $I V, P G C-1 \alpha$ : Peroxisome proliferator-activated receptor gamma coactivator 1-alpha, PGC-1 $\beta$ : Peroxisome proliferator-activated receptor gamma coactivator 1-beta, NRF1: Nuclear respiratory factor 1, ERR : Estrogen-related re-

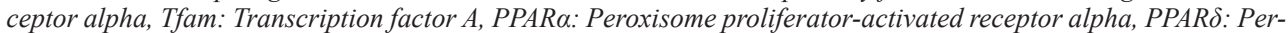
oxisome proliferator-activated receptor delta, SQSTM1: Sequestosome 1, PINK1: PTEN-induced kinase 1, PARK2: Parkin, FUNDC1: FUN14 domain containing 1, BNIP3: BCL2/adenovirus E1B 19 kDa protein-interacting protein 3, BNIP3L: BCL2/adenovirus E1B $19 \mathrm{kD}$ a protein-interacting protein 3-like and OPTN: Optineurin, GABARAPL1: GABA Type A Receptor Associated Protein Like 1, LC3A: Microtubule-associated protein 1 light chain 3 alpha, LC3B: Microtubule-associated protein 1 light chain 3 beta, Fis-1: Fission 1 protein, DNM1L: Dynamin-related protein 1, Mfn1: Mitofusin-1, Mfn2: Mitofusin-2, Opa1: optic atrophy protein 1 and Ndufb3: NADH oxidoreductase subunit B3. 


\section{Supplementary Table 2. Antibodies used for western blot}

\begin{tabular}{|l|l|l|l|l|}
\hline Target & RRID & Company & Product number & Dilution \\
\hline HKII & AB_2232946 & Cell Signaling Technology & Cat\#2867 & $1: 1000$ \\
\hline OXPHOS & AB_2629281 & MitoScience LLC & Cat\# MS604 & $1: 1000$ \\
\hline PGC-1 $\alpha$ & AB_10697773 & Millipore & Cat\# 516557 & $1: 1000$ \\
\hline NRF1 & AB_2154534 & Abcam & Cat\# ab55744 & $1: 1000$ \\
\hline ERR $\alpha$ & AB_1523580 & Abcam & Cat\# ab76228 & $1: 1000$ \\
\hline Tfam & AB_10682431 & Millipore & Cat\# DR1071 & $1: 1000$ \\
\hline SQSTM1 & AB_10624872 & Cell Signaling Technology & Cat\# 5114 & $1: 1000$ \\
\hline PINK1 & AB_10127658 & Novus Biologicals & Cat\# BC100-494 & $1: 2000$ \\
\hline PARK2 & AB_2159920 & Cell Signaling Technology & Cat\# 4211 & $1: 1000$ \\
\hline FUNDC1 & AB_10609242 & Santa Cruz Biotechnology & Cat\# sc-133597 & $1: 500$ \\
\hline BNIP3 & AB_2259284 & Cell Signaling Technology & Cat\# 3769S & $1: 1000$ \\
\hline BNIP3L & AB_2688036 & Cell Signaling Technology & Cat\# 12396 & $1: 1000$ \\
\hline GABARAPL1 & AB_2294415 & Proteintech Group & Cat\# 11010-1-AP & $1: 1000$ \\
\hline LC3B & AB_915950 & Cell Signaling Technology & Cat\# 2775 & $1: 1000$ \\
\hline DNM1L & AB_10950498 & Cell Signaling Technology & Cat\# 8570 & $1: 1000$ \\
\hline
\end{tabular}

HKII: Hexokinase II, OXPHOS: Oxidative phosphorylation, antibody cocktail (containing NADH: Ubiquinone oxidoreductase subunit B8 (NDUFB8), Succinate dehydrogenase complex, subunit B (SDHB), ubiquinol cytochrome c reductase core protein 2 (UQCRC2), Mitochondrially encoded cytochrome c oxidase I (mt-COI), ATP synthase, $H^{+}$ transporting, mitochondrial F1 complex, alpha (ATP5A)), PGC-1 $\alpha$ : Proliferative activated receptor gamma, coactivator 1 alpha, NRF1: Nuclear respiratory factor 1, ERRQ: Estrogen Related Receptor alpha, Tfam: Mitochondrial transcription factor A, SQSTM1: Sequestosome 1, PINK1: PTEN-induced kinase 1, PARK2: Parkin, FUNDC1: FUN14 domain-containing protein 1, BNIP3: BCL2/Adenovirus E1B $19 \mathrm{kDa}$ protein-interacting protein 3, GABARAPL1: $\gamma$-aminobutiric acid receptor-associated protein-like 1, LC3B: Microtubule associated protein 1A/1B-light chain 3 beta and DNM1L: Dynamin 1 Like. 



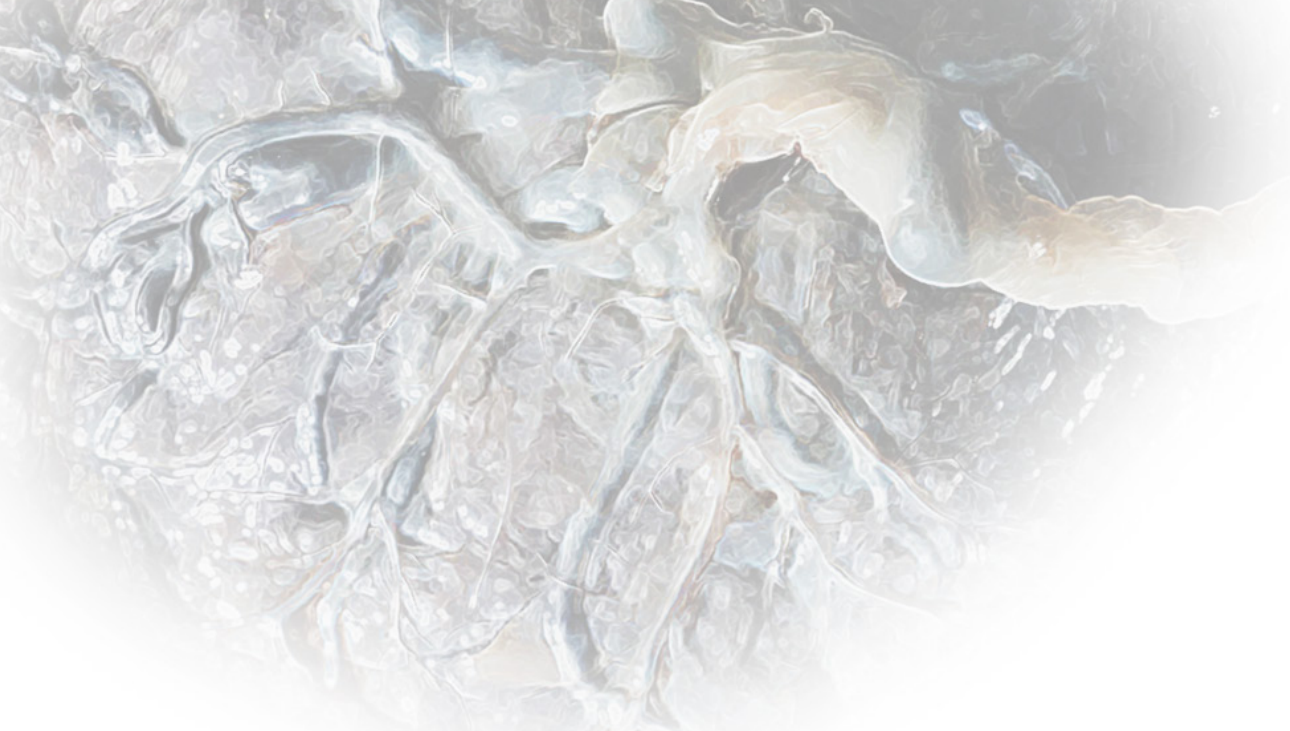




\section{Chapter 4}

\section{Aypoxia-induced mitochondrial abnormalities in cells of the placenta}

Philippe Vangrieken, Salwan Al-Nasiry, Aalt Bast, Pieter A. Leermakers, Christy B.M. Tulen, Ger. M.J. Janssen, Iris Kaminski, Iris Geomini, Titus Lemmens, Paul M.H. Schiffers, Frederik J. van Schooten, Alex H.V. Remels

Submidded. 


\section{Abstract}

\section{Introduction}

Impaired utero-placental perfusion is a well-known feature of early preeclampsia and is associated with placental hypoxia and oxidative stress. Although aberrations at the level of the mitochondrion have been implicated in PE pathophysiology, whether or not hypoxia-induced mitochondrial abnormalities contribute to placental oxidative stress is unknown.

\section{Methods}

We explored whether abnormalities in mitochondrial metabolism contribute to hypoxia-induced placental oxidative stress by using both healthy term placentae as well as a trophoblast cell line (BeWo cells) exposed to hypoxia. Furthermore, we explored the therapeutic potential of the antioxidants MitoQ and quercetin in preventing hypoxia-induced placental oxidative stress.

\section{Results}

Placental hypoxia was associated with increased oxidative stress, inflammation, and apoptosis. Moreover, experiments with MitoQ revealed that hypoxia-induced reactive oxygen species originated from the mitochondria in the trophoblasts. Also, in both placental explants as well as BeWo cells, hypoxia resulted in reductions in mitochondrial content, decreased abundance of key molecules involved in the electron transport chain and increased expression and activity of glycolytic enzymes. Furthermore, expression levels of key regulators of mitochondrial biogenesis were decreased while the abundance of constituents of the mitophagy, autophagy and mitochondrial fission machinery was increased in response to hypoxia.

\section{Conclusion}

This study is the first to demonstrate that placental hypoxia is associated with mitochondrial-generated reactive oxygen species and significant alterations in the molecular pathways controlling mitochondrial content and function. Furthermore, our data indicate that targeting mitochondrial oxidative stress may have therapeutic benefit in the management of pathologies related to placental hypoxia. 


\section{Introduction}

Preeclampsia (PE) is a progressive multisystem pregnancy disorder that affects $2 \%$ to $8 \%$ of all pregnancies (1). PE is mainly characterized by new onset of hypertension and proteinuria, caused by placental dysfunction. Although systemic pathological changes usually resolve following delivery of the placenta, it still predisposes mothers to cardiovascular, renal, and chorionic hypertensive disease in later life (1, 2). Currently, there is no treatment and management of PE predominantly involves stabilization of the mother and fetus with timely delivery to prevent deterioration of the condition and subsequently limit morbidity and mortality as a result of the disorder (2).

Although the pathogenesis of PE remains poorly understood, the current paradigm revolves around oxidative stress in the trophoblast, the cell type that forms the epithelial lining of the placental villi that are in direct contact with the maternal blood $(3,4)$. Oxidative stress has been shown to induce apoptosis in trophoblasts, accelerate trophoblast turn-over and promote excessive release of placental cytotoxic factors including e.g. pro-inflammatory cytokines and vasoactive compounds in the maternal circulatory system (5). These cytotoxic factors subsequently drive PE-like symptoms including endothelial dysfunction, systemic hypertension and end-organ hypo-perfusion $(5,6)$. Different stressors can lead to perturbation of placental endocrine actions of which the predominant one in PE is utero-placental malperfusion secondary to abnormal remodeling of the uterine spiral arteries $(7,8)$.

Disturbed delivery of blood, causing local hypoxia, is known to cause damage to the mitochondrial electron transport chain (ETC) which, as the ETC is the main site for intracellular oxygen consumption in the cell, can lead to increased production of reactive oxygen species (ROS) $(2,5)$. Interestingly, recent evidence suggests abnormalities at the level of the mitochondrion in PE-complicated placentae (9). However, whether or not hypoxia triggers these abnormalities and if this is linked to the development of oxidative stress in the placenta remains unclear.

Key mechanisms controlling mitochondrial function and content include biogenesis of new organelles (mitochondrial biogenesis), selective autophagy-mediated degradation of mitochondria (mitophagy) as well as mitochondrial fusion and fission events $(10,11)$. Mitochondrial biogenesis is essentially controlled by the peroxisome proliferator-activated receptor (PPAR) gamma coactivator 1 (PGC-1) molecules that co-activate different nuclear proteins with transcriptional activity such as estrogen-related receptors (ERRs), PPARs, and nuclear respiratory factor $1 / 2 \alpha$ (NRF- 
$1 / 2 \alpha$ ), and mitochondrial transcription factor A (Tfam). These proteins collectively drive the expression of mitochondrial proteins and replication of mitochondrial DNA (mtDNA) (12). Mitophagy can be divided in receptor-mediated and ubiquitin-mediated mitophagy. During receptor-mediated mitophagy, mitochondrial-based receptor proteins e.g. FUND14 domain containing 1 (FUNDC1), BCL2/adenovirus E1B 19 $\mathrm{kDa}$ protein interacting protein 3 (BNIP3) or BNIP3 like (BNIP3L), are activated. Subsequently, these activated receptor proteins recruit and bind a mammalian homologue of yeast autophagy-related protein 8 , including microtubule-associated protein 1 light chain 3 beta (LC3B) and gamma-aminobutyric acid type A receptor associated protein like 1 (GABARAPL1) and so predestines the organelle for authophagic degradation. The ubiquitin-mediated mitophagy pathway is initiated by accumulation of PTEN induced putative kinase 1 (PINK1) on the mitochondrial membrane, where they recruit E3 ubiquitin-protein ligase Parkin (PARK2) and/or several autophagy receptors (e.g. optineurin (OPTN)). When activated, PARK2 is able to ubiquitinate several different mitochondrial substrates, including mitofusin 1 (Mfn1) and Mfn2, which in turn recruit the LIR-containing autophagy adaptor protein sequestome-1 (SQSTM1) and LC3B resulting in degradation of the organelle.

Unravelling the relationship between hypoxia, the regulatory pathways controlling mitochondrial content and function, and oxidative stress in the trophoblast will enhance our insight into the complex pathogenesis of PE and may pave the way for new treatment modalities. Therefore, in this study, we exposed placental villous explants from non-complicated pregnancies as well as a trophoblast cell line (BeWo cells) to hypoxia and comprehensively assessed multiple indices of mitochondrial health, cellular oxidative stress, and antioxidant systems. Parameters related to (the molecular regulation of) mitochondrial health included assessment of transcript and protein abundance as well as activity levels of constituents of mitochondrial metabolic pathways and key regulators of mitochondrial biogenesis, mitophagy, and the mitochondrial fusion and fission machinery. In addition, the potential of the flavonoid quercetin (13) as well as the mitochondrial-targeted antioxidant MitoQ to limit oxidative stress in BeWo cells was investigated. 


\section{Materials and methods}

\section{Clinical subject characteristics}

Human term ( $>38$ weeks gestational age) placentae were collected from caesarean deliveries performed by the Department of Obstetrics and Gynaecology at the Maastricht University medical Center+. For this study, three placentae were used. Exclusion criteria were hypertension (pre-existing or onset during pregnancy) and/ or proteinuria as defined by the International Society for the study of Hypertension in Pregnancy (ISSHP). Approval was obtained from the Medical Ethics Committee Maastricht University Medical Center+ and all procedures on placentae were performed in accordance with the guidelines for experiments on human material (Maastricht University Medical Center+, Maastricht, the Netherlands, No. 16-4047). Oral informed consent was obtained from participants prior to the inclusion of the placenta.

\section{Villous explant isolation}

As described earlier by Vangrieken et al. (14), three placental villous explants from non-complicated caesarean deliveries were processed directly after delivery. Villous explants were collected from the central region of the placentae at the maternal side. The basal plate of the specimens was removed and the remaining tissue was rinsed in a HEPES solution $\left(\mathrm{NaCl} 143.3 \mathrm{mM}, \mathrm{KCl} 4.7 \mathrm{mM}, \mathrm{MgSO}_{4} 1.2 \mathrm{mM}, \mathrm{KH}_{2} \mathrm{PO}_{4}\right.$ $1.2 \mathrm{mM}, \mathrm{CaCl}_{2} 2.5 \mathrm{mM}$, glucose $5.5 \mathrm{mM}$, and HEPES $15 \mathrm{mM}$ ). Subsequently, per placenta, two villous explants of $30 \mathrm{~g}$ were collected and transferred into a $200 \mathrm{ml}$ pre-warmed $\left(37^{\circ} \mathrm{C}\right)$ HEPES buffer, kept in a warm water bath $\left(37^{\circ} \mathrm{C}\right)$ and aerated under a standard culture condition $\left(21 \% \mathrm{O}_{2}\right)$ or a hypoxic condition $\left(1 \% \mathrm{O}_{2}\right)$ for $3 \mathrm{~h}$. Tissue was collected and snap-frozen in liquid nitrogen, crushed by a mortar while frozen and stored at $-80{ }^{\circ} \mathrm{C}$ until use.

\section{Cell culture}

BeWo cells, a trophoblast cell line (ECACC, Porton Down, Salisbury, UK, No 86082803) were cultured according to the manufacture's protocol. Briefly, BeWo cells were cultured in Ham's F12 (Kaighn's) growth medium (Glutamine $2 \mathrm{mM}$ and D-Glucose $7 \mathrm{mM}$, GIBCO, Carlsbad, CA, USA) enriched with $10 \%$ (vol/vol) foetal bovine serum (FBS) (SIGMA, St. Louis, MO, USA) and antibiotics (50 U/ml penicillin and $50 \mu \mathrm{g} / \mathrm{ml}$ streptomycin; both from GIBCO). Cells were routinely maintained in $175-\mathrm{cm}^{2}$ falcon flasks at $\mathrm{pH} 7.4$ under $5 \% \mathrm{CO}_{2}$ and $95 \%$ humidity at $37{ }^{\circ} \mathrm{C}$ and passaged when reaching confluency of $70-80 \%$. For enzyme activity assays and RNA/DNA isolation, $24 \mathrm{~h}$ after cell seeding in 6-wells plates $(3 \times 105$ cells/well in 2 $\mathrm{ml}$ growth medium), cells were cultured for $24 \mathrm{~h}$ under a normoxic (control) condi- 
tion $\left(21 \% \mathrm{O}_{2}, / 5 \% \mathrm{CO}_{2}\right)+/$ - quercetin ( $3 \mu \mathrm{M}$, Sigma, St. Louis, MO, USA) or MitoQ (mitoquinone mesylate) $(1,4$ or $8 \mu \mathrm{M}$, MedChemExpress, New Jersey, USA) or a hypoxic condition $\left(1 \% \mathrm{O}_{2} / 5 \% \mathrm{CO}_{2}\right)+/$ - quercetin $(3 \mu \mathrm{M})$ or MitoQ $(1,4$ or $8 \mu \mathrm{M})$. For detection of intracellular ROS levels, $72 \mathrm{~h}$ after cell seeding, cells were cultured for $24 \mathrm{~h}$ in black and clear bottom-96-wells plates (Costar/sigma Aldrich) (1.5x104 cells/well in $200 \mu$ growth medium) under a normoxic (control) condition $\left(21 \% \mathrm{O}_{2}\right.$, $\left.15 \% \mathrm{CO}_{2}\right)+/$ - quercetin $(3 \mu \mathrm{M})$ or MitoQ $(1,4$ or $8 \mu \mathrm{M})$, or a hypoxic condition $(1 \%$ $\left.\mathrm{O}_{2} / 5 \% \mathrm{CO}_{2}\right)+/$ - quercetin $(3 \mu \mathrm{M})$, MitoQ $(1,4$ or $8 \mu \mathrm{M})$ or $\mathrm{H}_{2} \mathrm{O}_{2}(100 \mu \mathrm{l}$, positive control).

\section{Villous explant and BeWo viability}

Lactate dehydrogenase (LDH) activity, as an indicator of cell/tissue viability was measured in supernatant of both the cultured villous explants isolated from all 3 placentae and BeWo cells of the standard culture and the hypoxic condition using the cytotoxicity detection kit (Cat. No.11644793001, Roche, Mannheim, Germany).

\section{Preparation of the lysates}

Approximately $40 \mathrm{mg}$ of powdered placental tissue was homogenized for $10 \mathrm{sec}$ at maximal speed with a hand-held PRO Scientific Bio-Gen PRO200 homogenizer in $800 \mu \mathrm{KPE}$ lysis buffer $\left(13 \mathrm{mM} \mathrm{KH}_{2} \mathrm{PO}_{4}, 68 \mathrm{mM} \mathrm{K}_{2} \mathrm{HPO}_{4}, 9 \mathrm{mM}\right.$ EDTA, and 1\% Triton X-100) for antioxidant enzyme activity assays or in $800 \mu \mathrm{l}$ SET buffer (250 $\mathrm{mM}$ sucrose, $2 \mathrm{mM}$ EDTA, $10 \mathrm{mM}$ Tris, $\mathrm{pH}$ 7.4) for metabolic enzyme activity assays. For the generation of BeWo lysates, cells were washed twice with pre-cooled 1X Hank's balanced salt solution (HBSS), incubated for $15 \mathrm{~min}$ on ice in $500 \mu \mathrm{KPE}$ lysis buffer or in $100 \mu \mathrm{l}$ SET buffer and collected using a rubber policeman. Subsequently, samples were homogenized for $10 \mathrm{sec}$ at maximal speed with a handheld PRO Scientific Bio-Gen PRO200 homogenizer. KPE homogenates were centrifuged at $20,000 \mathrm{x} \mathrm{g}$ for $10 \mathrm{~min}$ at $4{ }^{\circ} \mathrm{C}$. Subsequently, lysates $(300 \mu \mathrm{l})$ were stored at $-80^{\circ} \mathrm{C}$ for the assessment of Trolox Equivalent Antioxidant Capacity (TEAC). The remaining lysate was mixed with $2.6 \%$ bovine serum albumin (BSA) (1:1) and stored at -80 ${ }^{\circ} \mathrm{C}$ for the determination of glutathione disulfide/glutathione (GSSG/GSH) levels. SET homogenates were snap-frozen in liquid nitrogen, defrosted, incubated on ice for $30 \mathrm{~min}$ and subsequently centrifuged at $20,000 \mathrm{x}$ g for $2 \mathrm{~min}$ at $4{ }^{\circ} \mathrm{C} .5 \% \mathrm{BSA}$ was added to the lysate $(1: 4)$ and stored at $-80^{\circ} \mathrm{C}$ for the assessment of the citrate synthase (CS), $\beta$-hydroxyacyl-CoA dehydrogenase (HADH) and phosphofructokinase (PFK) activity. For DNA and RNA isolation, BeWo cells were washed twice with pre-cooled $1 \mathrm{X}$ HBSS, incubated for $15 \mathrm{~min}$ on ice in $500 \mu \mathrm{l}$ Trizol reagent and further processed according to the manufacturer's protocol (Catalog Number 15596026 and 15596018, Invitrogen TM, USA). For generation of whole cell lysates (for western 
blot analysis), BeWo cells were washed twice with pre-cooled $1 \mathrm{X}$ HBSS, incubated for $30 \mathrm{~min}$ on ice in $100 \mu \mathrm{l}$ IP lysis buffer (50 mM Tris, $150 \mathrm{mM} \mathrm{NaCl}, 10 \%$ glycerol, 0.5\% Nonidet P40, $1 \mathrm{mM}$ EDTA, $1 \mathrm{mM} \mathrm{Na} \mathrm{VO}_{4}, 5 \mathrm{mM} \mathrm{NaF}, 10 \mathrm{mM} \beta$-glycerophasphate, $1 \mathrm{mM} \mathrm{Na} \mathrm{O}_{7} \mathrm{P}_{2}, 1 \mathrm{mM}$ DTT, $10 \mu \mathrm{g} / \mu \mathrm{l}$ leupeptin, $1 \%$ apropeptin, $1 \mathrm{mM}$ PMSF, pH 7.4) and collected using a rubber policeman. Subsequently, samples were homogenized for $20 \mathrm{sec}$ at maximal speed using the homogenizer. Lysates were incubated for $30 \mathrm{~min}$ on ice and centrifuged at $20,000 \mathrm{x}$ g for $30 \mathrm{~min}$ at $4{ }^{\circ} \mathrm{C}$. Lysates were aliquoted $(1 \mu \mathrm{g} / \mu \mathrm{l})$ in Laemmli buffer $(0.25 \mathrm{M}$ Tris- $\mathrm{HCl}, 8 \%$ (w/v) SDS, 40\% (v/v) glycerol, 0.4 M DTT, 0.04\% (w/v) Bromphenol Blue, pH 6.8) and boiled for $5 \mathrm{~min}$ at $95{ }^{\circ} \mathrm{C}$. Protein concentrations of whole cell lysates and enzyme activity lysates were determined using the PierceTM BCA Protein Assay kit according to the manufacturer's protocol (Pierce Chemical Co., Rockford, IL).

\section{Citrate synthase (CS) activity}

As previously described (CS; EC 2.3.3.1) (15), a reaction mix was set up in 96 well plates in duplicate containing $5 \mu \mathrm{l}$ undiluted sample, $200 \mu \mathrm{l}$ reagent containing Tris (100 mM), DNTB $(0.1 \mathrm{mM})$ and acetyl-coenzym A $(0.3 \mathrm{mM})$. The reaction was started with $5 \mu \mathrm{l}$ start reagent containing oxaloacetic acid $(25 \mathrm{mM})$. Enzyme activity was monitored at $412 \mathrm{~nm}\left(37^{\circ} \mathrm{C}\right)$ and corrected for total protein concentration.

\section{B-hydroxyacyl-CoA dehydrogenase (HADH) activity}

As previously described (HADH; EC 1.1.1.35) (16), a reaction mix was set up in a 96 well plate in duplicate containing $10 \mu \mathrm{l}$ undiluted sample, $100 \mu \mathrm{l}$ reagent containing NADH $(1.1 \mathrm{mM})$, tetrapotassium pyrophosphate $(100 \mathrm{mM})$. The reaction was started with $10 \mu \mathrm{l}$ acetyl-coenzym A (2.4 mM). Enzyme activity was kinetically-monitored at $340 \mathrm{~nm}\left(37^{\circ} \mathrm{C}\right)$ and corrected for total protein concentration.

\section{Phosphofructokinase (PFK) activity}

A reaction mix was set up in a 96 well plate in duplicate containing $20 \mu 1$ undiluted sample, $100 \mu \mathrm{l}$ reagent containing Tris Base (49.6 mM), $\mathrm{MgCl}_{2} \cdot 6 \mathrm{H}_{2} \mathrm{O}(7.4 \mathrm{mM}), \mathrm{KCl}$ (3.2 mM), KCN (384.6 $\mu \mathrm{M})$, ATP (3.0 mM), DTT (1.5 mM), NADH (0.3 mM), aldolase $(0.019 \mathrm{U})$, glycerol-3-phosphate dehydrogenase $(0.019 \mathrm{U})$ and triose phosphate, isomerase $(0.019 \mathrm{U}), \mathrm{pH}$ 8.0. The reaction was started with fructose-6-phosphate $(35.9 \mathrm{mM})$ in Tris buffer $(49.6 \mathrm{mM}), \mathrm{pH}$ 8.0. Enzyme activity was monitored at 340 $\mathrm{nm}\left(37^{\circ} \mathrm{C}\right)$ and corrected for total protein concentration.

\section{Quantitative real-time PCR}

cDNA was synthesized from 400 ng RNA using the iScriptTM cDNA synthesis kit (Bio-Rad, Laboratories, Hercules, CA, USA) according to the manufacturer's proto- 
col. $4.4 \mu \mathrm{l}$ of 1:50 diluted cDNA was used for quantitative PCR amplification using target-specific primers (Supplementary Table 1) and 2X SensimixTM SYBER ${ }^{\circledR}$ \& Fluorescein mix (Bioline, Alphen aan de Rijn, the Netherlands) on a LightCycler480 384-wells PCR machine (Roche, Almere, the Netherlands). Specificity of PCR amplification was checked by melt curve analysis. Expression levels of genes of interest were corrected using normalization factor calculated based on the expression of 2 different housekeeping genes (Cyclophilin A and RPL13A), which were found to be most stable from a selection of 3 genes by using the GeNorm software (Primerdesign, Southamton, USA). The list of primers can be found in Supplementary Table 1.

\section{Mitochondrial DNA (mtDNA) copy number}

An amount of $4.4 \mu \mathrm{l}$ of 1:25 diluted DNA was used for qPCR as described above, using mitochondrial DNA (mtDNA, Cytochrome c oxidase subunit II (COXII)) and genomic DNA (gDNA, Ribosomal Protein L13a (RPL13A)) - specific primers (Supplementary Table 1). mtDNA/gDNA ratio was determined by dividing the relative quantity of mtDNA by the relative quantity of gDNA.

\section{Western Blotting}

As described earlier by Leermakers et al. (17), $10 \mu \mathrm{g}$ of protein was run through a Criterion XT 4-12 or 12\% Bis-Tris gel (Bio-Rad, Veenendaal, the Netherlands) in 1x MES running buffer (Bio-Rad, Veenendaal, the Netherlands) at 100 volts, and was subsequently blotted on a Nitrocellulose membrane (Bio-Rad Laboratories B.V., Veenendaal, the Netherlands) by electroblotting. At least two protein ladders were loaded on each gel (Precision Plus Protein ${ }^{\mathrm{TM}}$ All Blue Standards \#161-0373, Bio-Rad Laboratories, Veenendaal, the Netherlands). Membranes were stained with $0.2 \%$ PonceauS in $1 \%$ acetic acid (Sigma-Aldrich, Zwijndrecht, the Netherlands) for 5 min, washed with milliQ and imaged using the Amersham ${ }^{\mathrm{TM}}$ Imager 600 (GE Healthcare, Eindhoven, the Netherlands) to quantify total protein content as correction for gel-loading. Membranes were blocked for $1 \mathrm{~h}$ with Tween20 Tris-buffered saline (TBST; $20 \mathrm{mM}$ Tris, $137 \mathrm{mM} \mathrm{NaCl}, 0.1 \%$ (vol/vol) Tween20, pH 7.6) containing 3\% (w/v) non-fat dry milk (Campina, Eindhoven, the Netherlands), washed, and incubated overnight at $4{ }^{\circ} \mathrm{C}$ with a target-specific primary antibody (Supplementary Table 2) diluted 1:1,000-1:10,000 in TBST with 3\% (w/v) BSA or non-fat dry milk at $4{ }^{\circ} \mathrm{C}$. Subsequently, membranes were washed and incubated with a HRP-conjugated secondary antibody (\#BA-9200, \#BA-1000, Vector Laboratories, Amsterdam, the Netherlands), diluted 1:10,000 in 3\% (w/v) non-fat dry milk in TBST for $1 \mathrm{~h}$ at room temperature. Membranes were then washed, incubated for 5 min with $0.5 \mathrm{x}$ SuperSignal West PICO or 0.25x West Femto Chemiluminescent Substrate (Thermo Scientific, Landsmeer, the Netherlands) and imaged using the Amersham ${ }^{\mathrm{TM}}$ Imag- 
er 600. Original unaltered images were quantified with Image Quant software (GE Healthcare, Eindhoven, the Netherlands). Measured protein quantity was corrected for total protein content. Images included in the figures of this manuscript have been adjusted for brightness and contrast equally throughout the picture.

\section{Glutathione disulfide/glutathione (GSSG/GSH) levels}

GSH $(0.1-10 \mu \mathrm{M})$ and GSSG $(0.1-5 \mu \mathrm{M})$ standards were prepared in KPE buffer and $1.3 \% 5$-sulfosalicylic acid. GSSG standards and samples were diluted 1:10 with neat 2-vinylpyridine, incubated and mixed for $1 \mathrm{~h}$ to form a stable complex with GSH, preventing it from participating in the enzymatic recycling reaction with glutathione reductase. Reactions were set up in a 96 well plate and $50 \mu 1$ of the sample was loaded in duplicate. Reactions were initiated by adding $100 \mu \mathrm{l} 0.8 \mathrm{mM} \mathrm{NADPH} / 0.6$ $\mathrm{mM}$ DTNB 1:1 and $4 \mathrm{U} / \mathrm{ml}$ GSSG reductase to the samples. Color development of the samples and standards was recorded kinetically for $3 \mathrm{~min}$ in 9 reads at $412 \mathrm{nM}$ resulting in GSH + GSSG and GSH slope values. Activity was corrected for protein content of the samples and expressed in $\mathrm{nM} / \mathrm{mg}$ protein $/ \mathrm{min}$.

\section{Trolox Equivalent Antioxidant Capacity (TEAC)}

First, a 5 mM 2,2'-azino-bis(3-ethylbenzothiazoline-6-sulphonic acid) (ABTS) solution was prepared in $145 \mathrm{mM}$ sodium phosphate buffer ( $\mathrm{pH}$ 7.4). Then, an ABTS ${ }^{-}$ solution was prepared by adding $10 \mu \mathrm{l} / 100$ horseradish peroxidase (HRP) and $10 \mu 1$ of $2 \mathrm{mM} \mathrm{H}_{2} \mathrm{O}_{2}$ solution and diluted in an ABTS solution to a final absorbance of 0.70 \pm 0.02 at $734 \mathrm{~nm}$ at $37^{\circ} \mathrm{C}$. Deproteinization of the samples was performed by adding $10 \%$ trichloroacetic acid (TCA) $(1: 1)$ to the samples. For measuring antioxidant capacity, $50 \mu \mathrm{l}$ of the lysate was mixed with $950 \mu \mathrm{ABTS} \mathrm{AB}^{-}$solution at $37^{\circ} \mathrm{C}$ for 5 min and absorbance was measured at $734 \mathrm{~nm}$ and compared to the absorbance of an ABTS $^{-}{ }^{-}$solution without sample. Absorbance was corrected for total protein content.

\section{Intracellular ROS levels}

Intracellular ROS levels were quantified using the 2',7'-dichlorodihydrofluorescein diacetate (DCFH-DA)-assay. $72 \mathrm{~h}$ after cell seeding, cells were washed twice with $150 \mu \mathrm{l}$ HBSS, incubated for $1 \mathrm{~h}$ with $100 \mu \mathrm{l}$ H2DCFH-DA $(50 \mu \mathrm{M})$ and washed twice again with $150 \mu \mathrm{l}$ HBSS. After $24 \mathrm{~h}$ culture under the different conditions in duplicate as described above, DCF was measured at $\lambda$ excitation $=485 \mathrm{~nm}$ and $\lambda$ emission $=525 \mathrm{~nm}$ and corrected for total protein content. 


\section{Statistical analysis}

Data are depicted as bar graphs indicating the mean and SEM as fold change compared to the control. For each comparison, the D'agrostino and Pearson omnibus normality test was used to test normality and subsequently either an unpaired t-test or Mann Whitney test was used accordingly (GraphPad Software, La Jolla California USA). A p-value $<0.05$ was considered as significantly different from the control group and was presented as follows: Ns: $p>0.05,{ }^{*} p \leq 0.05,{ }^{*} \mathrm{p} \leq 0.01,{ }^{* * *} \mathrm{p} \leq 0.001$. For each assay, three independent experiments were performed in which each experimental condition contained 3-12 biological replicates. For subsequent data analysis, replicates were expressed as fold change compared to the control and were subsequently pooled. 


\section{Results}

\section{Villous explant and BeWo viability}

No significant differences $(\mathrm{p}=0.4610)$ were found for LDH activity between the control- and hypoxic-conditioned villous explant medium indicating that viability of the placental villous explants was comparable between the two conditions after $3 \mathrm{~h}$ of incubation. Furthermore, when BeWo cells were cultured for $6 \mathrm{~h}, 24 \mathrm{~h}$ and $48 \mathrm{~h}$ under a normoxic or hypoxic condition, only at $48 \mathrm{~h}$ a significant increase $(\mathrm{p}=$ 0.004) in LDH activity was observed in cell supernatant of BeWo cells exposed to hypoxia compared to the control condition (data not shown).

\section{Increased oxidative stress levels in placental villous explants and BeWo cells upon hypoxia}

We first determined the impact of hypoxia $\left(1 \% \mathrm{O}_{2}\right)$ on oxidative stress levels and known cellular antioxidant defense systems in placental villous explants as well as in cultured BeWo cells. As depicted in Figure 1, both the glutathione disulfide/glutathione (GSSG/GSH) ratio as well as the trolox equivalent antioxidant capacity (TEAC) increased significantly in both placental villous explants and BeWo cells exposed to hypoxia ( $3 \mathrm{~h}$ and $24 \mathrm{~h}$ respectively). Also, although placental villous explants exposed to hypoxia did not show alterations in mRNA abundance of known antioxidant enzymes, in BeWo cells exposed to hypoxia mRNA transcript levels of catalase 1 (Cat1) and mitochondrial manganese-dependent superoxide dismutase 2 (MnSOD2) significantly increased while transcript levels of superoxide dismutase 1 (SOD1) significantly decreased. (Figure 1A-F).

As mitochondria are the main site for cellular oxygen consumption and the main source of cellular ROS production, we next investigated whether or not hypoxia-induced changes in ROS levels and antioxidant status originated from the mitochondria. Therefore, we used the mitochondrial-targeted antioxidant MitoQ in cultured BeWo cells during exposure to hypoxia. Intracellular ROS levels, as well as oxidized glutathione levels increased more than two-fold in BeWo cells exposed to hypoxia compared to normoxia-exposed BeWo cells, and MitoQ normalized both hypoxia-induced increases in intracellular ROS formation and oxidized glutathione levels in BeWo cells in a concentration-dependent manner (Fig 1G-H). In addition, as shown in Supplementary Figure 1, the antioxidant quercetin, did not reduce hypoxia-induced intracellular ROS formation. However, interestingly, the combination of the lowest concentration of MitoQ $(1 \mu \mathrm{M})$ and quercetin $(3 \mu \mathrm{M})$ significantly decreased hypoxia-induced intracellular ROS production and levels of oxidized glutathione to levels similar as in the normoxia-exposed BeWo cells. 


\section{Placental explants}
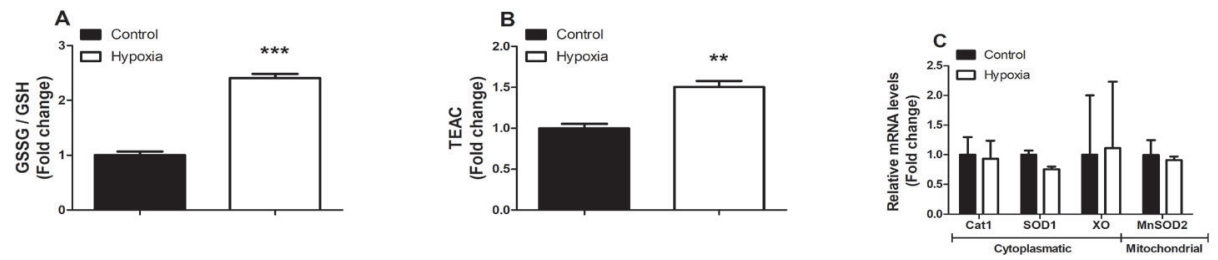

\section{Trophoblasts}
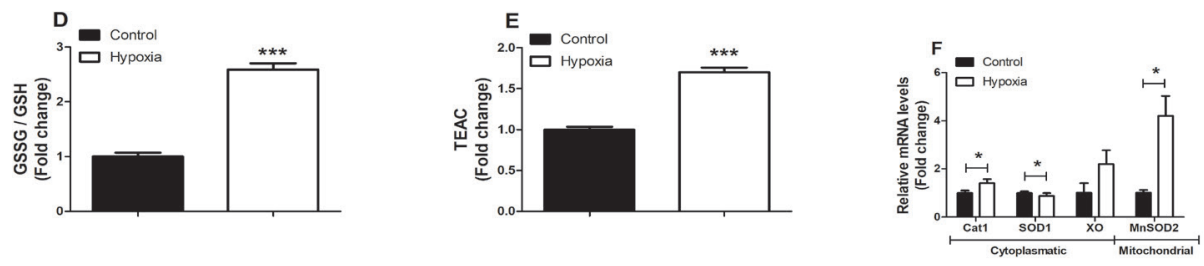
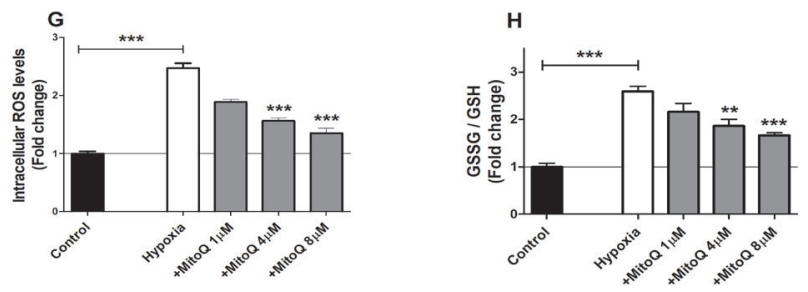

Figure 1. Increased oxidative stress levels in placentae and trophoblasts upon hypoxia. GSSG/ GSH ratio (A), TEAC (B) and transcript levels of CAT1, SOD1, XO and MnSOD2 (C) assessed in placental villous explants exposed to normoxia (control) or hypoxia for $3 \mathrm{~h}(\mathrm{n}=3)$ and GSSG/GSH ratio (D), TEAC (E) and transcript levels of CAT1, SOD1, XO and MnSOD2 (F) assessed in trophoblasts exposed to hypoxia or normoxia (control) for 24h. Intracellular ROS levels using DCFH-DA (G) and GSSG/GSH ratio $(\mathrm{H})$ were assessed in trophoblasts exposed to normoxia (control), hypoxia or hypoxia $+\operatorname{MitoQ}(1,4$ or $8 \mu \mathrm{M})$ (DCFH-DA-assay: $\mathrm{n}=6$-12/experimental condition ( $\mathrm{n}=3$ experiments), GSSG/ GSH-assay: $\mathrm{n}=2-6$ experimental condition ( $\mathrm{n}=3$ experiments)). Data are presented as fold change compared to the control and as mean with SEM. ${ }^{*} p \leq 0.05,{ }^{* *} \mathrm{p} \leq 0.01$ and ${ }^{* * *} \mathrm{p} \leq 0.001$. GSSG: Oxidized glutathione, GSH: Reduced glutathione, TEAC: Trolox equivalent antioxidant capacity, Cat1: Catalase-1, SOD1: Superoxide dismutase 1, XO: Xanthine oxidase, MnSOD2: Manganese-dependent superoxide dismutase and DCFH-DA: 2',7'-Dichlorodihydrofluorescein diacetate.

In addition to these indicators of oxidative stress, mRNA transcript levels of tumor necrosis factor alpha (TNF- $\alpha$ ), interleukin 6 (IL-6) and interleukin 8 (IL-8) as well as the pro-apoptotic mRNA transcript ratio of Bcl-2associated X protein / anti-apoptotic B-cell lymphoma 2 (BAX/BCL-2) were all significantly increased in BeWo cells in response to $24 \mathrm{~h}$ of hypoxia but were unaltered in placental villous explants after a $3 \mathrm{~h}$ exposure to hypoxia (Supplementary Figure 2A-D). 


\section{Hypoxia-induced decrease in mitochondrial content in placental villous explants and BeWo cells}

Having established that hypoxia exposure, in BeWo cells, results in mitochondrial ROS production and considering the fact that ROS can damage mtDNA (18), we next assessed indices of mitochondrial content in placental villous explants as well as in cultured BeWo cells exposed to hypoxia. As shown in Figure 2, hypoxia-induced a profound reduction in mitochondrial content in both placental villous explants as well as in cultured BeWo cells. This was evidenced by a reduction in mtDNA copy number and a decrease in the enzyme activity of citrate synthase (CS) (Figure 2AD), two well-described indicators of mitochondrial content (19). Moreover, the combination MitoQ and quercetin, in concentrations that completely normalized hypoxia-induced ROS formation and oxidized glutathione levels (Supplementary Figure 1A-B), ameliorated hypoxia-induced reductions in mtDNA copy number in BeWo cells (Figure 2E).

\section{Placental explants}
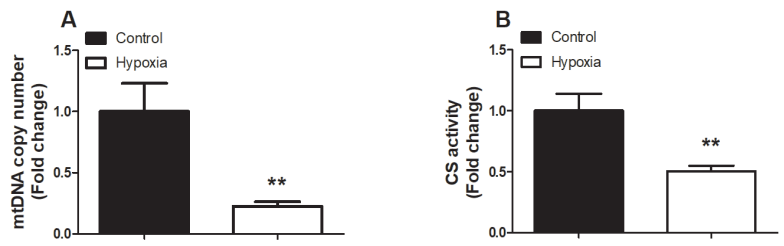

\section{Trophoblasts}
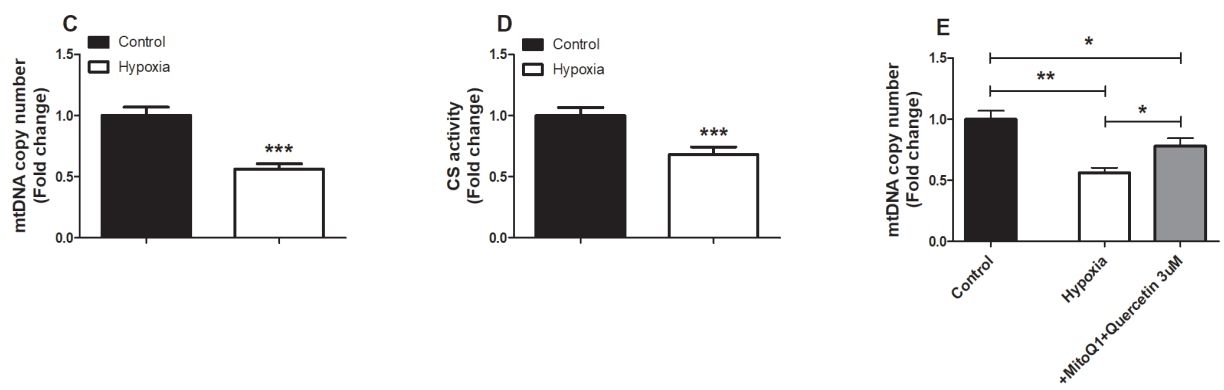

Figure 2. Lower mitochondrial content in placental villous explants and trophoblasts upon hypoxia. mtDNA copy number (A) and CS activity (B) assessed in placental villous explants exposed to hypoxia or normoxia (control) for $3 \mathrm{~h}(\mathrm{n}=3$ in duplicate) and mtDNA copy number (C) and CS activity (D) assessed in trophoblasts exposed to normoxia (control) or hypoxia for $24 \mathrm{~h}$. mtDNA copy number (E) was assessed in trophoblasts exposed to normoxia (control), hypoxia, hypoxia + MitoQ (1, 4 or 8 $\mu \mathrm{M})$, quercetin $(3 \mu \mathrm{M})$ or MitoQ $(1 \mu \mathrm{M})+$ quercetin $(3 \mu \mathrm{M})$ for $24 \mathrm{~h}(\mathrm{n}=3-6 /$ experimental condition $(\mathrm{n}=3$ experiments)). Data are presented as fold change compared to the control and as mean with SEM. ${ }^{*} \mathrm{p} \leq 0.05,{ }^{*} \mathrm{p} \leq 0.01$ and ${ }^{* * *} \mathrm{p} \leq 0.001$. mtDNA: Mitochondrial DNA and CS: Citrate synthase. 


\section{Increased abundance and activity of glycolytic constituents in placental cells in response to hypoxia}

Next, we investigated whether the decrease in mitochondrial content observed in placental villous explants and in BeWo cells exposed to hypoxia was associated with changes in (mitochondrial- and non-mitochondrial) metabolic processes. Therefore, we assessed the abundance and activity levels of key constituents of the ETC, fatty acid- $\beta$ oxidation (FAO) and glycolysis. mRNA abundance as well as enzymatic activity of $\beta$-hydroxyacyl-CoA dehydrogenase (HADH), a key enzyme involved in mitochondrial FAO, were unaltered in response to hypoxia in both placental villous explants as well as in BeWo cells (Supplementary Figure 3A-D). Interestingly, while in placental villous explants exposed to hypoxia for $3 \mathrm{~h}$ transcript and protein abundance of both nuclear- or mitochondrial-encoded sub-units of ETC complexes were unaltered (Supplementary Figure 4A-C), protein levels of ubiquinol cytochrome c reductase core protein 2 (UQCRC2, complex III) as well as mRNA transcript levels of all investigated sub-units, including mitochondrial-encoded COXII, were significantly decreased in BeWo cells after $24 \mathrm{~h}$ exposure to hypoxia (Supplementary Figure 4D-F).

When assessing activity levels of phosphofructokinase (PFK), a key enzyme involved in glycolysis, we observed that hypoxia resulted in a significant increase in PFK activity in both placental villous explants as well as in BeWo cells. Moreover, in BeWo cells, but not placental villous explants, this was associated with a significant increase in protein and mRNA transcript levels of hexokinase II (HKII) as well as transcript levels of the glucose transporter 1 (GLUT1) (Figure 3A-F).

\section{Placental explants}
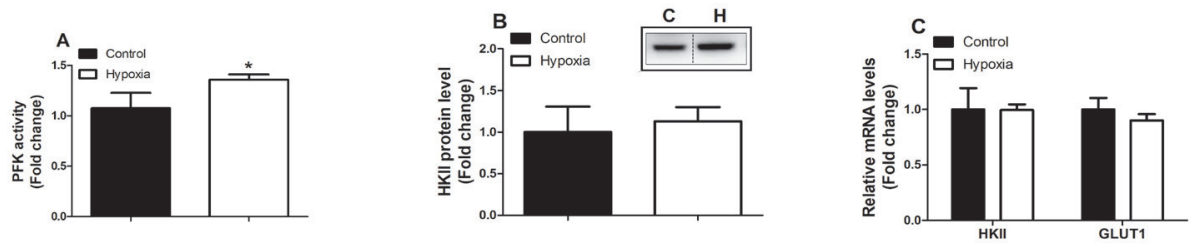

\section{Trophoblasts}
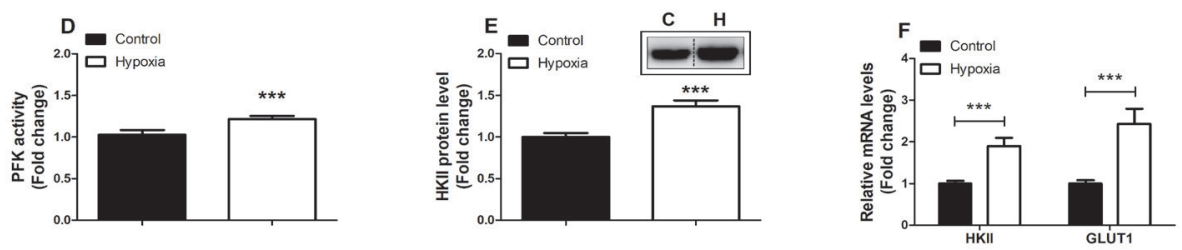

Figure 3. Increased expression and activity of key glycolytic enzymes in placentae and trophoblasts upon hypoxia. PFK enzyme activity (A), HKII protein level (B), mRNA expression levels of 
HKII and Glut-1 (C), assessed in placental villous explants exposed to normoxia (control) or hypoxia for $3 \mathrm{~h}(\mathrm{n}=3$ in duplicate (PFK assay) or ( $\mathrm{n}=3$ (protein and mRNA)) and PFK enzyme activity (D), HKII protein level (E), mRNA expression levels of HKII and Glut-1 (F), assessed in trophoblasts exposed to normoxia (control) or hypoxia for $24 \mathrm{~h}$ ( $\mathrm{n}=3-6 /$ experimental condition ( $\mathrm{n}=3$ experiments)). Representative immunoblots are shown and Western blots were corrected for total protein loading assessed by Ponceau S Staining with adjusted contrast equally applied to the whole photograph. Black boxes around the representative pictures indicate that they were cut from the same Western blot. Data are presented as fold change compared to the control and as mean with SEM. *p $\leq 0.05$ and ***p $\leq$ 0.001. PFK: Phosphofructokinase, HKII: Hexokinase and GLUT1: Glucose transporter 1.

\section{Alterations in the molecular regulation of mitochondrial biogen- esis in BeWo cells upon hypoxia}

Considering the reductions in mitochondrial content in placental cells upon exposure to hypoxia, we next explored whether hypoxia affected the molecular regulation of mitochondrial biogenesis by the PGC-1 signaling network. As shown in Figure 4, while no differences were observed in the abundance of key regulators of mitochondrial biogenesis in placental villous explants exposed to hypoxia for $3 \mathrm{~h}$ (Figure 4A-B), protein levels of Tfam and ERR $\alpha$ were significantly decreased in response to $24 \mathrm{~h}$ of hypoxia in BeWo cells. Furthermore, mRNA transcript levels of PGC-1 $\beta$, NRF2 $\alpha$, Tfam, and ERR $\alpha$ were significantly decreased in BeWo cells upon $24 \mathrm{~h}$ of exposure to hypoxia (Figure 4C-D).

\section{Placental explants}
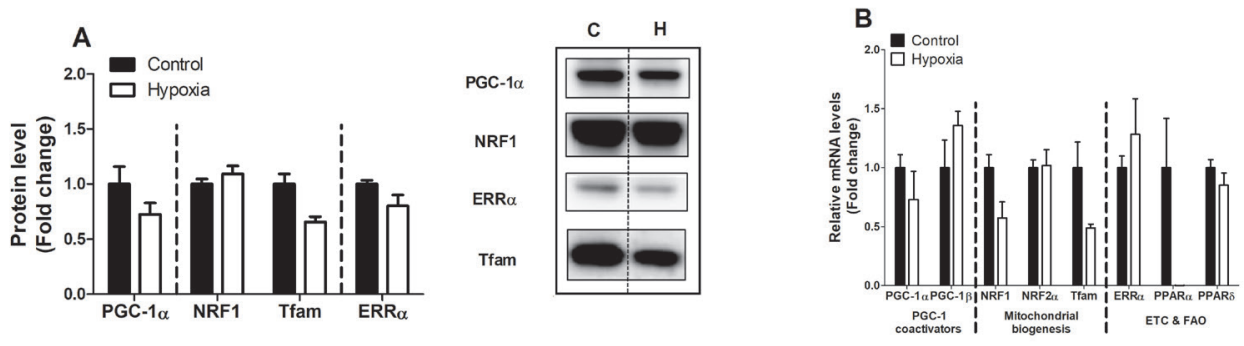

\section{Trophoblasts}
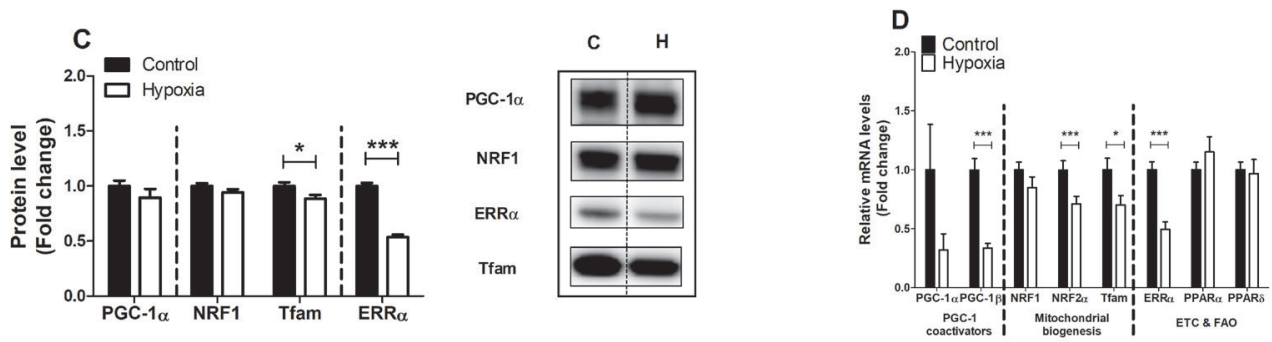

Figure 4. Alterations in the molecular regulation of mitochondrial biogenesis in trophoblasts upon hypoxia. Protein levels of PGC-1 $\alpha$, NRF1, Tfam and ERR $\alpha$ (A) and mRNA transcript levels of 
PGC-1 $\alpha$, PGC-1 $\beta$, NRF1, NRF2 $\alpha$, Tfam, ERR $\alpha$, PPAR $\alpha$ and PPAR $\delta$ (B) assessed in placental villous explants exposed to normoxia (control) or hypoxia for $3 \mathrm{~h}(\mathrm{n}=3)$ and protein levels of PGC-1 $\alpha$, NRF1, Tfam and ERR $\alpha(C)$ and mRNA transcript levels of PGC-1 $\alpha$, PGC-1 $\beta$, NRF1, NRF2 $\alpha$, Tfam, ERR $\alpha$, PPAR $\alpha$ and PPAR $\delta$ (D) assessed in trophoblasts exposed to normoxia (control) or hypoxia for $24 \mathrm{~h}(\mathrm{n}=$ 3-6/experimental condition ( $\mathrm{n}=3$ experiments)). Representative immunoblots are shown and Western blots were corrected for total protein loading assessed by Ponceau S Staining with adjusted contrast equally applied to the whole photograph. Black boxes around the representative pictures indicate that they were cut from the same Western blot. Data are presented as fold change compared to the control and as mean with SEM. ${ }^{*} p \leq 0.05$ and $* * * p \leq 0.001$. PGC- $1 \alpha$ : Peroxisome proliferator-activated receptor gamma coactivator 1-alpha, NRF1: Nuclear respiratory factor 1, Tfam: Mitochondrial transcription factor A, ERR $\alpha$ : Estrogen-related receptor alpha, PGC-1 $\beta$ : Peroxisome proliferator-activated receptor gamma coactivator 1-beta, NRF2 $\alpha$ : Nuclear respiratory factor 2 alpha, PPAR $\alpha$ : Peroxisome proliferator-activated receptor alpha and PPAR $\delta$ : Peroxisome proliferator-activated receptor delta.

\section{Expression of key constituents of the mitophagy machinery is altered in BeWo cells upon hypoxia}

As mitochondrial content is determined by the balance between mitochondrial biogenesis and breakdown through mitophagy (e.g. mitochondrial-specific autophagy), we next assessed the impact of hypoxia on key constituents of the mitophagy machinery in placental villous explants and BeWo cells. As mitophagy requires several general autophagy-related proteins for generating the autophagosomal membrane and priming the autophagosome to the mitochondria, these proteins were studied as well.

All investigated constituents of the autophagy- (Supplementary Figure 5A-B) and mitophagy machinery (Figure 5A-B) were unaltered in placental villous explants exposed for $3 \mathrm{~h}$ to hypoxia compared to the control condition. In contrast, in BeWo cells exposed for $24 \mathrm{~h}$ to hypoxia, mitophagy-related protein levels of PINK1 and FUNDC1, as well as mRNA transcript levels of FUNDC1, were significantly decreased in response to hypoxia while both protein and mRNA transcript levels of BNIP3 and BNIP3L were potently increased. In addition, while PARK2 protein levels were not affected in BeWo cells exposed to hypoxia, mRNA transcript levels of PARK2 were significantly increased in response to hypoxia (Figure 5C-D). Also, in cultured BeWo cells, $24 \mathrm{~h}$ of hypoxia resulted in a significant decrease in autophagy-associated LC3BI and LC3BII protein levels while the ratio of LC3BII/LC3BI was significantly increased compared to the normoxic condition. In addition, while both protein as well as mRNA levels of GABARAPL were increased in response to hypoxia, protein levels of SQSTM1 were decreased and its transcript levels increased upon hypoxia (Supplementary Figure 5C-D). OPTN mRNA levels increased 2-fold in response to hypoxia (Supplementary Figure 5D). 


\section{Placental explants}
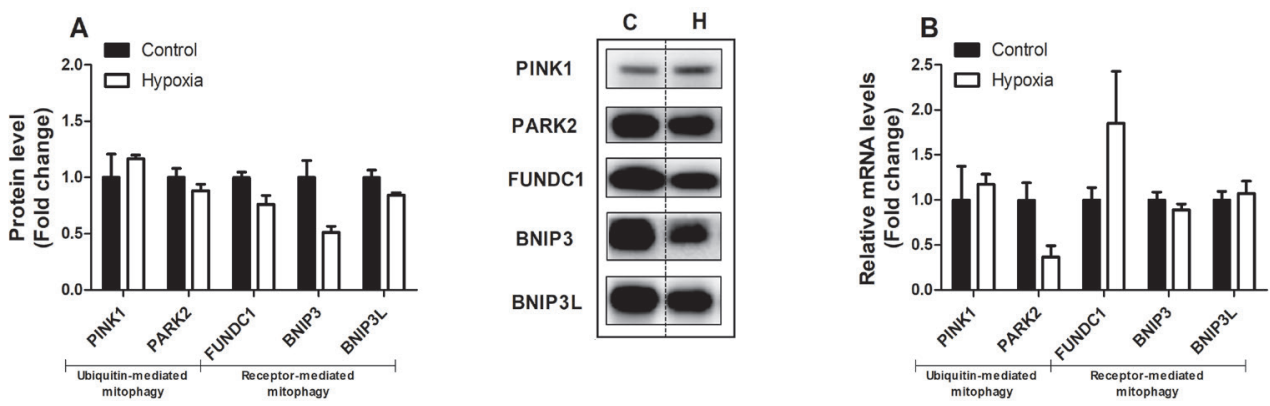

\section{Trophoblasts}
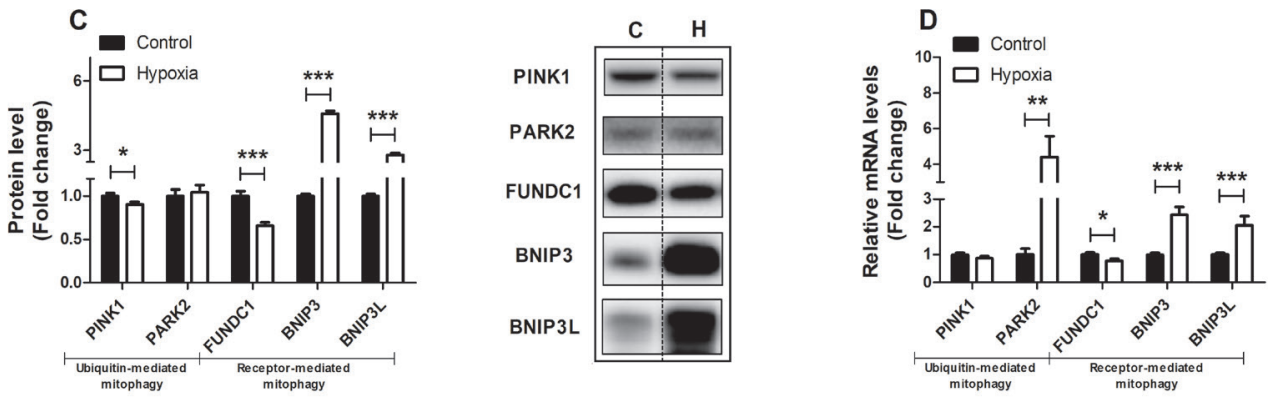

Figure 5. Expression of key constituents of the mitophagy machinery is altered in trophoblasts upon hypoxia. Mitophagy-associated protein levels of PINK1, PARK2, FUNDC1, BNIP3 and BNIP3L (A) and mRNA transcript levels of PINK1, PARK2, FUNDC1, BNIP3 and BNIP3L (B) assessed in placental villous explants exposed to normoxia (control) or hypoxia for $3 \mathrm{~h}(\mathrm{n}=3)$ and mitophagy-associated protein levels of PINK1, PARK2, FUNDC1, BNIP3 and BNIP3L (C) and mRNA transcript levels of PINK1, PARK2, FUNDC1, BNIP3 and BNIP3L (D) assessed in trophoblasts exposed to normoxia (control) or hypoxia for $24 \mathrm{~h}(\mathrm{n}=3-6 /$ experimental condition $(\mathrm{n}=3$ experiments)). Representative immunoblots are shown and Western blots were corrected for total protein loading assessed by Ponceau S Staining with adjusted contrast equally applied to the whole photograph. Black boxes around the representative pictures indicate that they were cut from the same Western blot. Data are presented as fold change compared to the control and as mean with SEM. ${ }^{*} \mathrm{p} \leq 0.05,{ }^{* *} \mathrm{p} \leq 0.01$ and ${ }^{* * *} \mathrm{p} \leq 0.001$. PINK1: PTEN-induced kinase 1, PARK2: E3 ubiquitin-protein ligase Parkin, FUNDC1: FUN14 domain containing 1, BNIP3: BCL2/adenovirus E1B $19 \mathrm{kDa}$ protein-interacting protein 3 and BNIP3L: BCL2/adenovirus E1B $19 \mathrm{kDa}$ protein-interacting protein 3-like.

\section{Alterations of key constituents of mitochondrial fission and fu- sion in BeWo cells upon hypoxia}

As mitochondrial fission and fusion are key events in the processes of mitophagy and mitochondrial biogenesis and are essential in maintaining normal mitochondrial homeostasis, mRNA and protein levels of mitochondrial fission and fusion proteins were investigated both in placental villous explants as well as BeWo cells exposed 
to hypoxia. As depicted in Figure 6A-B, no alterations were found in fission and fusion-related proteins and mRNA transcript levels in placental villous explants exposed for $3 \mathrm{~h}$ to hypoxia. In BeWo cells, fission related protein levels of dynamin1-like protein (DNM1L), a key protein involved in mitochondrial fission (20), was significantly decreased after $24 \mathrm{~h}$ exposure to hypoxia. In addition, fission-related mRNA transcript levels of fission 1 (Fis1) and DNM1L were significantly increased after exposure to hypoxia, while mRNA expression of fusion-related transcript levels of mitofusin-1 (Mfn1) and Mfn2 were reduced in BeWo cells upon exposure to hypoxia (Figure 6C-D).

\section{Placental explants}
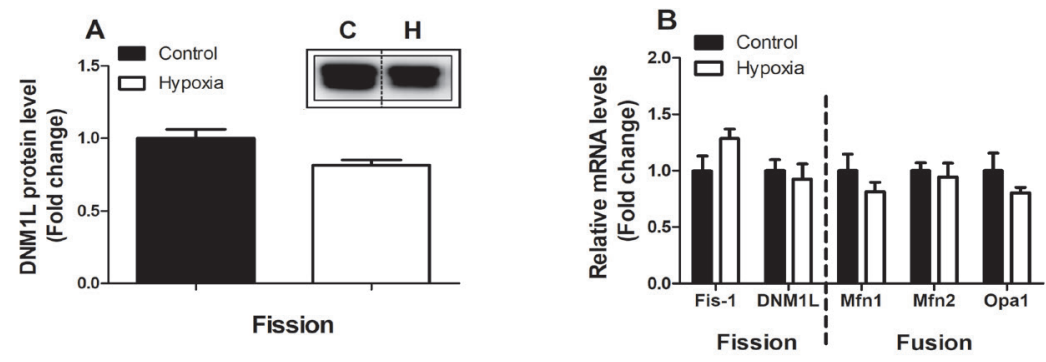

Trophoblasts
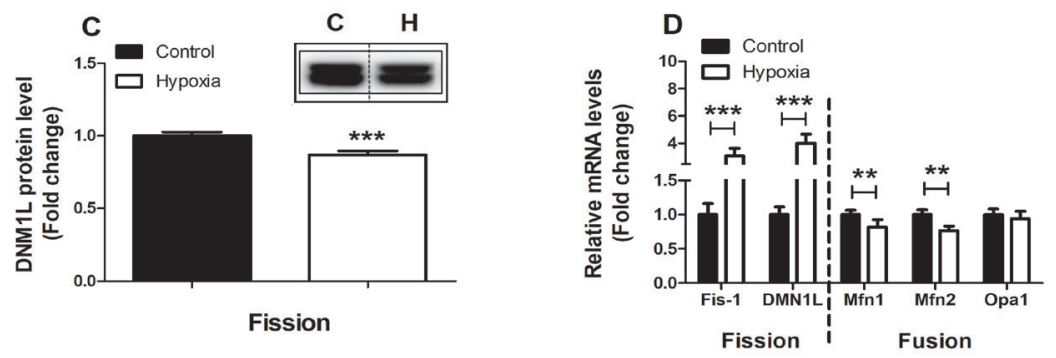

Figure 6. Alterations of key constituents of mitochondrial fission and fusion in trophoblasts upon hypoxia. Fission-related protein levels of DNM1L (A), fission-related mRNA transcript levels of Fis1 and DNM1L and fusion-related mRNA transcript levels of Mfn1, Mfn2 and Opa1 (B) assessed in placentae exposed to normoxia (control) or hypoxia for $3 \mathrm{~h}(\mathrm{n}=3)$ and fission-related protein levels of DNM1L (C), fission-related mRNA transcript levels of Fis-1 and DNM1L and fusion-related mRNA transcript levels of Mfn1, Mfn2 and Opa1 (D) assessed in trophoblasts exposed to normoxia (control) or hypoxia for $24 \mathrm{~h}(\mathrm{n}=3-6 /$ experimental condition $(\mathrm{n}=3$ experiments)). Representative immunoblots are shown and Western blots were corrected for total protein loading assessed by Ponceau S Staining with adjusted contrast equally applied to the whole photograph. Black boxes around the representative pictures indicate that they were cut from the same Western blot. Data are presented as fold change compared to the control and as mean with SEM. ${ }^{* *} p \leq 0.01$ and $* * * p \leq 0.001$. DNM1L: Dynamin-1like protein, Fis-1: Fission 1 protein, Mfn1: Mitofusin-1, Mfn2: Mitofusin-2 and Opa1: Optic atrophy protein 1 . 


\section{Discussion}

In the current study, we show that hypoxia triggers placental oxidative stress, which likely resulted from ROS formation and alterations in antioxidant status in BeWo cells. Moreover, we show for the first time that, in BeWo cells exposed to hypoxia, mitochondria are a major source of ROS and that hypoxia triggers a profound loss of mitochondrial content. Furthermore, in response to hypoxia, the abundance and activity of key constituents of glycolysis rapidly increased which, upon prolonged exposure to hypoxia, was also associated with significant reductions in the abundance of subunits of the electron transport chain and changes in the expression of key regulatory molecules controlling mitochondrial biogenesis, mitophagy and mitochondrial fission and fusion events.

Placental villous explants were exposed to hypoxia for up to $3 \mathrm{~h}$ since previous studies using placental villous explants ex vivo showed that viability of first and third trimester tissue in culture could only be maintained for $4 \mathrm{~h}$ (21). Longer exposure times lead to pronounced tissue deterioration and massive apoptosis of the trophoblasts in placental villous explants, indicating that it is only suitable to study acute effects (22). To study the effects of long-term exposure to hypoxia and to determine if changes are trophoblast-specific, BeWo cells were used. Since LDH viability between the control and hypoxic condition was significantly different after $48 \mathrm{~h}$ culturing, $24 \mathrm{~h}$ exposure to hypoxia was used in this study.

The presence of placental oxidative stress and the link with PE pathophysiology is well-established. Indeed, there is abundant evidence that placental oxidative stress triggers the release of cytotoxic and vasoactive factors as well as pro-inflammatory cytokines into the maternal circulation driving PE symptoms like maternal endothelial dysfunction and hypertension (2). One of the key triggers of placental oxidative stress that has been proposed in PE is hypoxia $(5,23)$. In support of this notion, stabilization of hypoxia-inducible factor 1-alpha (HIF-1 $\alpha$ ) has been reported in PE and hypoxia-exposed placentae (24-26). Furthermore, both in hypoxia-exposed trophoblasts as well as in a prenatal hypoxia rat model for PE, increased oxidative stress has been reported $(5,23,27)$. In line with these observations, the GSSG/GSH ratio and intracellular ROS levels in our study were significantly increased in placental cells in response to hypoxia. Furthermore, hypoxia markedly increased total antioxidant capacity in placental cells compared to the control group in our study, which may be a compensatory response to increased oxidative stress $(28,29)$. The fact that mitochondrial superoxide concentration is tightly controlled by the mitochondrial antioxidant MnSOD2 (30), combined with the notion that hypoxia in our study re- 
sulted in a 4-fold increase in transcript levels of MnSOD2 suggested mitochondria to be a significant source of ROS. In agreement with this observation, the mitochondrial-targeted antioxidant MitoQ concentration-dependently reduced intracellular ROS levels and lowered the GSSG/GSH ratio in BeWo cells during hypoxia in our study. This is in line with previous finding by Aljunaidy et al. (23), who showed not only a normalization of oxidative stress levels by MitoQ in a hypoxic rat model of reduced placental perfusion, but also protection against sex- and age-dependent alterations in cardiac and vascular health of offspring later in life. In addition to oxidative stress, hypoxia in our study resulted in increased expression of inflammatory mediators in BeWo cells as well as an increased BAX/BCL-2 ratio, indicative of activation of apoptosis. In line with this, it is well-established that both inflammation and apoptosis of placental cells are induced by hypoxia and are implicated in PE pathophysiology. Indeed, women with PE show higher plasma levels of TNF- $\alpha$ and elevated TNF- $\alpha$ protein and mRNA levels in their placentae compared to normal pregnant women, (30). Furthermore, TNF- $\alpha$ is known to be a potent stimulus for endothelin 1 secretion, which is known to be a major vasoactive compound released by the placenta under hypoxia, contributing to maternal hypertension $(14,31,32)$. With regard to apoptosis, an increased BAX/BCL-2 ratio was also associated with an increased abundance of BNIP3 proteins, which act as an activator for hypoxia-induced cell apoptosis and autophagy by triggering the BAX/Bak or LC-3/Beclin 1 signalling pathway $(33,34)$. Several Bcl-2 family members including BAX, have been shown to be directly regulated by a lack of oxygen via stabilization of HIF-1 (35).

Interestingly, besides their traditional role in cellular energy metabolism, mitochondria are important mediators of oxidative stress, inflammation, and apoptosis. Moreover, evidence is emerging that PE is associated with abnormalities at the levels of the mitochondrion in the placenta $(36,37)$. Indeed, besides alterations in placental mitochondrial content in PE (38), several human studies showed significant reductions in adenosine triphosphate (ATP) levels in PE placentae, which is suggestive of impaired mitochondrial functioning (39-41). Indicative of a role for hypoxia in mediating these changes, exposure of mice to lower oxygen $\left(13 \%\right.$ instead of $\left.21 \% \mathrm{O}_{2}\right)$ led to an overall reduction in placental mitochondrial oxidative capacity (42) which could be beneficial to keep $\mathrm{O}_{2}$ transfer to the foetus constant (43). This is in line with our findings as we reported evidence for significant reductions in mitochondrial content in both healthy placentae and BeWo cells exposed to hypoxia. Furthermore, expression of complex III of the ETC, which is the major source of mitochondrial production ROS in hypoxic conditions (44), as well as transcript levels of complex I and IV including mitochondrial-encoded COXII were decreased in BeWo cells exposed to hypoxia, which is in concert with earlier studies $(45,46)$. In addition, hypoxia in 
placental cells was associated with increased key glycolytic enzyme activity in our study, which together with the above-described data are suggestive for a shift from oxidative to glycolytic energy metabolism. In agreement with these findings, exposure to low oxygen led to an increased trans-placental transfer of glucose and amino acids in mice (47). Moreover, oxidative stress during hypoxia is known to activate HIF which is known to increase transcription of glycolytic enzymes and promote anaerobic glycolysis to maintain ATP synthesis under low oxygen conditions (48). In accordance with our results, showing increased transcription of GLUT1 upon hypoxia, GLUT1 is known to be activated upon binding of HIF- $1 \alpha$ to the hypoxia-responsive element in the GLUT-1 promotor, which has been observed earlier in both hypoxia-exposed and PE placentae $(5,49)$. Collectively, our data, in concert with available literature suggest that in response to hypoxia, BeWo cells quickly develop mitochondrial-generated oxidative stress, display an acute increase in glycolysis followed by a decrease in expression of key components of the ETC.

Reductions in oxidative respiration in hypoxic tissues are generally associated with decreased mitochondrial biogenesis (50-52). In line with this and with decreased mitochondrial content in placental cells in response to hypoxia, we observed significant reductions in expression levels of key regulators of mitochondrial biogenesis, which is in accordance with recent studies (34). Besides mitochondrial biogenesis, selective autophagy of mitochondria (i.e. mitophagy) is involved in the regulation of mitochondrial content in cells. Enhanced mitophagy is suggested to be an adaptive response to reduce the levels of ROS and protect cell integrity during hypoxia. It has previously been found that $1 \% \mathrm{O}_{2}$ can lower mitochondrial mass up to $75 \%$ through the initiation of the HIF1-dependent expression of BNIP3, a mitochondrial protein that competes with $\mathrm{Bcl} 2$ and thereby freeing beclin1 to trigger autophagy (53). In our study, BeWo cells exposed to hypoxia showed an increased abundance of receptor-mediated mitophagy-related proteins BNIP3 and BNIP3L, which is in agreement with a previous study by Knyazev et al. (34). In line with the literature, hypoxia-induced increases in protein levels of BNIP3 and BNIP3L in BeWo cells were indeed associated with an increased abundance of general autophagy-related GABARAPL1 and an increased ratio of LC3BII/LC3BI protein levels, which are known to predispose mitochondria for degradation $(54,55)$. In addition, abundance of receptor-mediated mitophagy-related protein FUNDC1 was significantly decreased in BeWo cells in response to hypoxia, which is in line with the hypoxia-mediated degradation of FUNDC1 as described by Chen et al. (56). Collectively, our data indicate that receptor-mediated mitophagy in placentae is activated upon hypoxia and may well contribute to the loss of mitochondrial content that we observed. In addition, mitochondrial biogenesis and mitophagy are highly integrated 
with fusion and fission events. Increased mitochondrial fission has been implicated in the pathogenesis of several diseases and is often linked with the increased presence of oxidative stress and apoptosis (57-60). In this regard, increased levels of fission-related Fis-1 and DMN1L transcripts and decreased levels of fusion-related Mfn1 and Mfn2 transcripts indicate that the balance between fusion and fission may be tilted towards increased mitochondrial fission in response to hypoxia in our study. Collectively, with regard to the time-course of hypoxia-induced changes, our data show that oxidative stress in cells of the placenta develops rapidly in response to hypoxia, which is associated with profound reductions in mitochondrial content and increased activity of glycolytic enzymes. Alterations in the molecular mechanisms controlling mitochondrial content and function (mitochondrial biogenesis vs mitophagy), however, only become apparent after prolonged exposure to hypoxia (24h).

Interestingly, although MitoQ and quercetin together initiated complete normalization of intracellular ROS formation during hypoxia, MitoQ alone did not completely prevent intracellular ROS formation suggesting that other cellular ROS-generating systems contribute to hypoxia-induced oxidative stress in placental cells. In agreement with this, xanthine oxidase has been shown to be implicated in PE pathophysiology and has been suggested to contribute to (hypoxia-induced) placental oxidative stress $(61,62)$.

\section{Conclusion}

Our results show hypoxia-induced abnormalities at the level of the mitochondrion and the molecular pathways controlling mitochondrial content and function in placentae and oxidative stress, which may contribute to PE pathogenesis or other hypoxia-related pregnancy disorders like intrauterine growth restriction (IUGR). Although the concept of hypoxia-induced mitochondrial abnormalities in cells of the placenta is not new (63), our study is the first to show that hypoxia-induced oxidative stress mainly originates from the mitochondria and is unique in comprehensively assessing not only indices of mitochondrial content but also multiple constituents of mitochondrial biogenesis and mitophagy in cells of the placenta in response to hypoxia.

\section{Acknowledgements}

This study was supported by NUTRIM Graduate Program. There was no additional external funding received for this study. 


\section{References}

1. August P, Sibai BM. Preeclampsia: Clinical features and diagnosis. UpToDate Accessed December. 2017;22.

2. Chiarello DI, Abad C, Rojas D, Toledo F, Vazquez CM, Mate A, et al. Oxidative stress: Normal pregnancy versus preeclampsia. Biochim Biophys Acta Mol Basis Dis. 2018. Epub 2018/12/28. doi: 10.1016/j.bbadis.2018.12.005. PubMed PMID: 30590104.

3. Burton G, Woods A, Jauniaux E, Kingdom J. Rheological and physiological consequences of conversion of the maternal spiral arteries for uteroplacental blood flow during human pregnancy. Placenta. 2009;30(6):473-82.

4. Vangrieken P, Vanterpool SF, van Schooten FJ, Al-Nasiry S, Andriessen P, Degreef $\mathrm{E}$, et al. Histological villous maturation in placentas of complicated pregnancies. Histol Histopathol. 2020:18205. doi: 10.14670/HH-18-205. PubMed PMID: 31985030 .

5. Tong W, Giussani DA. Preeclampsia link to gestational hypoxia. J Dev Orig Health Dis. 2019:1-12. Epub 2019/04/11. doi: 10.1017/S204017441900014X. PubMed PMID: 30968806.

6. Aouache R, Biquard L, Vaiman D, Miralles F. Oxidative Stress in Preeclampsia and Placental Diseases. Int J Mol Sci. 2018;19(5). Epub 2018/05/19. doi: 10.3390/ijms19051496. PubMed PMID: 29772777; PubMed Central PMCID: PMCPMC5983711.

7. Bronson SL, Bale TL. The placenta as a mediator of stress effects on neurodevelopmental reprogramming. Neuropsychopharmacology. 2016;41(1):207.

8. Burton GJ, Woods AW, Jauniaux E, Kingdom JC. Rheological and physiological consequences of conversion of the maternal spiral arteries for uteroplacental blood flow during human pregnancy. Placenta. 2009;30(6):473-82. Epub 2009/04/21. doi: 10.1016/j.placenta.2009.02.009. PubMed PMID: 19375795; PubMed Central PMCID: PMCPMC2697319.

9. Holland OJ, Cuffe JSM, Dekker Nitert M, Callaway L, Kwan Cheung KA, Radenkovic F, et al. Placental mitochondrial adaptations in preeclampsia associated with progression to term delivery. Cell Death Dis. 2018;9(12):1150. Epub 2018/11/21. doi: 10.1038/s41419-018-1190-9. PubMed PMID: 30455461; PubMed Central PMCID: PMCPMC6242930.

10. Palikaras K, Tavernarakis N. Mitochondrial homeostasis: the interplay between mitophagy and mitochondrial biogenesis. Experimental gerontology. 2014;56:182-8.

11. Ashrafi G, Schwarz T. The pathways of mitophagy for quality control and clearance of mitochondria. Cell death and differentiation. 2013;20(1):31. 
12. Scarpulla RC. Metabolic control of mitochondrial biogenesis through the PGC-1 family regulatory network. Biochimica et Biophysica Acta (BBA)-Molecular Cell Research. 2011;1813(7):1269-78.

13. Ebegboni VJ, Dickenson JM, Sivasubramaniam SD. Antioxidative effects of flavonoids and their metabolites against hypoxia/reoxygenation-induced oxidative stress in a human first trimester trophoblast cell line. Food chemistry. 2019;272:11725 .

14. Vangrieken P, Al-Nasiry S, Janssen GMJ, Weseler AR, Spaanderman ME, Bast A, et al. The direct and sustained consequences of severe placental hypoxia on vascular contractility. PLoS One. 2018;13(8):e0202648. Epub 2018/08/25. doi: 10.1371/journal.pone.0202648. PubMed PMID: 30142162; PubMed Central PMCID: PMCPMC6108468.

15. Shepherd D, Garland PB. The kinetic properties of citrate synthase from rat liver mitochondria. Biochem J. 1969;114(3):597-610. Epub 1969/09/01. doi: 10.1042/bj1140597. PubMed PMID: 5820645; PubMed Central PMCID: PMCPMC1184933.

16. Bergmeyer H, Gawehn K, Grassl M. 3-Hydroxyacyl-CoA dehydrogenase. Methods of enzymatic analysis. 1974;1:474.

17. Leermakers P, Schols A, Kneppers A, Kelders M, de Theije C, Lainscak M, et al. Molecular signalling towards mitochondrial breakdown is enhanced in skeletal muscle of patients with chronic obstructive pulmonary disease (COPD). Scientific reports. 2018;8(1):15007.

18. Nissanka N, Moraes CT. Mitochondrial DNA damage and reactive oxygen species in neurodegenerative disease. FEBS letters. 2018;592(5):728-42.

19. Larsen S, Nielsen J, Hansen CN, Nielsen LB, Wibrand F, Stride N, et al. Biomarkers of mitochondrial content in skeletal muscle of healthy young human subjects. J Physiol. 2012;590(14):3349-60. Epub 2012/05/16. doi: 10.1113/jphysiol.2012.230185. PubMed PMID: 22586215; PubMed Central PMCID: PMCPMC3459047.

20. Cai M, He P, Fang DL. Hypoxia-induced mitochondrial translocation of DNM1L increases mitochondrial fission and triggers mPTP opening in HCC cells via activation of HK2. Oncology reports. 2019;42(3):1125-32.

21. Sooranna SR, Oteng-Ntim E, Meah R, Ryder TA, Bajoria R. Characterization of human placental explants: morphological, biochemical and physiological studies using first and third trimester placenta. Hum Reprod. 1999;14(2):536-41. Epub 1999/04/01. doi: 10.1093/humrep/14.2.536. PubMed PMID: 10100006.

22. Di Santo S, Malek A, Sager R, Andres AC, Schneider H. Trophoblast viability in perfused term placental tissue and explant cultures limited to 7-24 hours. Placenta. 2003;24(8-9):882-94. Epub 2003/09/18. doi: 10.1016/s0143-4004(03)00142- 
5. PubMed PMID: 13129686.

23. Aljunaidy MM, Morton JS, Kirschenman R, Phillips T, Case CP, Cooke C-LM, et al. Maternal treatment with a placental-targeted antioxidant (MitoQ) impacts offspring cardiovascular function in a rat model of prenatal hypoxia. Pharmacological research. 2018;134:332-42.

24. Iwagaki S, Yokoyama Y, Tang L, Takahashi Y, Nakagawa Y, Tamaya T. Augmentation of leptin and hypoxia-inducible factor 1alpha mRNAs in the pre-eclamptic placenta. Gynecol Endocrinol. 2004;18(5):263-8. Epub 2004/09/07. PubMed PMID: 15346662 .

25. Rajakumar A, Doty K, Daftary A, Harger G, Conrad KP. Impaired oxygen-dependent reduction of HIF-1alpha and -2alpha proteins in pre-eclamptic placentae. Placenta. 2003;24(2-3):199-208. Epub 2003/02/05. PubMed PMID: 12566247.

26. Nevo O, Soleymanlou N, Wu Y, Xu J, Kingdom J, Many A, et al. Increased expression of sFlt-1 in in vivo and in vitro models of human placental hypoxia is mediated by HIF-1. American Journal of Physiology-Regulatory, Integrative and Comparative Physiology. 2006;291(4):R1085-R93.

27. Malek A, Sager R, Schneider H. Effect of hypoxia, oxidative stress and lipopolysaccharides on the release of prostaglandins and cytokines from human term placental explants. Placenta. 2001;22:S45-S50.

28. Llurba E, Gratacos E, Martin-Gallan P, Cabero L, Dominguez C. A comprehensive study of oxidative stress and antioxidant status in preeclampsia and normal pregnancy. Free Radical Bio Med. 2004;37(4):557-70. doi: 10.1016/j.freeradbiomed.2004.04.035. PubMed PMID: WOS:000223053600013.

29. Shea TB, Rogers E, Ashline D, Ortiz D, Sheu M-S. Quantification of antioxidant activity in brain tissue homogenates using the 'total equivalent antioxidant capacity'. Journal of neuroscience methods. 2003;125(1-2):55-8.

30. Hung TH, Burton GJ. Hypoxia and reoxygenation: a possible mechanism for placental oxidative stress in preeclampsia. Taiwan J Obstet Gynecol. 2006;45(3):189200. Epub 2006/12/19. doi: 10.1016/S1028-4559(09)60224-2. PubMed PMID: 17175463.

31. LaMarca BB, Cockrell K, Sullivan E, Bennett W, Granger JP. Role of endothelin in mediating tumor necrosis factor-induced hypertension in pregnant rats. Hypertension. 2005;46(1):82-6. Epub 2005/06/02. doi: 10.1161/01. HYP.0000169152.59854.36. PubMed PMID: 15928030.

32. LaMarca B, Speed J, Fournier L, Babcock SA, Berry H, Cockrell K, et al. Hypertension in response to chronic reductions in uterine perfusion in pregnant rats: effect of tumor necrosis factor-alpha blockade. Hypertension. 2008;52(6):11617. Epub 2008/11/05. doi: 10.1161/HYPERTENSIONAHA.108.120881. PubMed PMID: 18981324; PubMed Central PMCID: PMCPMC2788766. 
33. Xu Z, Jin X, Cai W, Zhou M, Shao P, Yang Z, et al. Proteomics Analysis Reveals Abnormal Electron Transport and Excessive Oxidative Stress Cause Mitochondrial Dysfunction in Placental Tissues of Early-Onset Preeclampsia. PROTEOMICS-Clinical Applications. 2018;12(5):1700165.

34. Knyazev EN, Zakharova GS, Astakhova LA, Tsypina IM, Tonevitsky AG, Sukhikh GT. Metabolic Reprogramming of Trophoblast Cells in Response to Hypoxia. Bull Exp Biol Med. 2019;166(3):321-5. Epub 2019/01/11. doi: 10.1007/ s10517-019-04342-1. PubMed PMID: 30627907.

35. Sowter HM, Ratcliffe PJ, Watson P, Greenberg AH, Harris AL. HIF-1-dependent regulation of hypoxic induction of the cell death factors BNIP3 and NIX in human tumors. Cancer Res. 2001;61(18):6669-73. PubMed PMID: WOS:000171108400010.

36. Afroze SH, Kalagiri RR, Reyes M, Zimmerman JD, Beeram MR, Drever N, et al. Apoptotic and stress signaling markers are augmented in preeclamptic placenta and umbilical cord. BBA clinical. 2016;6:25-30.

37. Gathiram P, Moodley J. Pre-eclampsia: its pathogenesis and pathophysiolgy. Cardiovascular journal of Africa. 2016;27(2):71.

38. Mandò C, De Palma C, Stampalija T, Anelli GM, Figus M, Novielli C, et al. Placental mitochondrial content and function in intrauterine growth restriction and preeclampsia. American Journal of Physiology-Endocrinology and Metabolism. 2013;306(4):E404-E13.

39. Zhou X, Han T-L, Chen H, Baker PN, Qi H, Zhang H. Impaired mitochondrial fusion, autophagy, biogenesis and dysregulated lipid metabolism is associated with preeclampsia. Experimental cell research. 2017;359(1):195-204.

40. Yu J, Guo X, Chen R, Feng L. Downregulation of mitofusin 2 in placenta is related to preeclampsia. BioMed Research International. 2016;2016.

41. Padmini E, Lavanya S, Uthra V. Preeclamptic placental stress and over expression of mitochondrial HSP70. Clinical chemistry and laboratory medicine. 2009;47(9):1073-80.

42. Sferruzzi-Perri AN, Higgins JS, Vaughan OR, Murray AJ, Fowden AL. Placental mitochondria adapt developmentally and in response to hypoxia to support fetal growth. Proc Natl Acad Sci U S A. 2019;116(5):1621-6. Epub 2019/01/19. doi: 10.1073/pnas.1816056116. PubMed PMID: 30655345; PubMed Central PMCID: PMCPMC6358710.

43. Postigo L, Heredia G, Illsley NP, Torricos T, Dolan C, Echalar L, et al. Where the $\mathrm{O} 2$ goes to: preservation of human fetal oxygen delivery and consumption at high altitude. The Journal of physiology. 2009;587(3):693-708.

44. Guzy RD, Schumacker PT. Oxygen sensing by mitochondria at complex III: the paradox of increased reactive oxygen species during hypoxia. Exp Physi- 
ol. 2006;91(5):807-19. Epub 2006/07/22. doi: 10.1113/expphysiol.2006.033506. PubMed PMID: 16857720.

45. Muralimanoharan S, Maloyan A, Mele J, Guo C, Myatt LG, Myatt L. MIR210 modulates mitochondrial respiration in placenta with preeclampsia. Placenta. 2012;33(10):816-23.

46. Wang Z, Zhang G, Lin M. Mitochondrial tRNA(leu)(UUR) gene mutation and the decreased activity of cytochrome c oxidase in preeclampsia. J Tongji Med Univ. 1999;19(3):209-11. Epub 2003/07/05. PubMed PMID: 12840896.

47. Higgins JS, Vaughan OR, Fernandez de Liger E, Fowden AL, Sferruzzi-Perri AN. Placental phenotype and resource allocation to fetal growth are modified by the timing and degree of hypoxia during mouse pregnancy. J Physiol. 2016;594(5):134156. Epub 2015/09/18. doi: 10.1113/JP271057. PubMed PMID: 26377136; PubMed Central PMCID: PMCPMC4771776.

48. Tissot van Patot MC, Murray AJ, Beckey V, Cindrova-Davies T, Johns J, Zwerdlinger L, et al. Human placental metabolic adaptation to chronic hypoxia, high altitude: hypoxic preconditioning. American Journal of Physiology-Regulatory, Integrative and Comparative Physiology. 2009;298(1):R166-R72.

49. Rajakumar A, Conrad KP. Expression, ontogeny, and regulation of hypoxia-inducible transcription factors in the human placenta. Biol Reprod. 2000;63(2):55969. Epub 2000/07/25. doi: 10.1095/biolreprod63.2.559. PubMed PMID: 10906065. 50. Jose C, Bellance N, Rossignol R. Choosing between glycolysis and oxidative phosphorylation: a tumor's dilemma? Biochimica et Biophysica Acta (BBA)-Bioenergetics. 2011;1807(6):552-61.

51. Zhang H, Gao P, Fukuda R, Kumar G, Krishnamachary B, Zeller KI, et al. HIF-1 inhibits mitochondrial biogenesis and cellular respiration in VHL-deficient renal cell carcinoma by repression of C-MYC activity. Cancer cell. 2007;11(5):40720 .

52. Schönenberger MJ, Kovacs WJ. Hypoxia signaling pathways: modulators of oxygen-related organelles. Frontiers in cell and developmental biology. 2015;3:42.

53. Saito S, Nakashima A. Review: The role of autophagy in extravillous trophoblast function under hypoxia. Placenta. 2013;34 Suppl:S79-84. Epub 2013/01/12. doi: 10.1016/j.placenta.2012.11.026. PubMed PMID: 23306070.

54. Wei H, Liu L, Chen Q. Selective removal of mitochondria via mitophagy: distinct pathways for different mitochondrial stresses. Biochim Biophys Acta. 2015;1853(10 Pt B):2784-90. Epub 2015/04/04. doi: 10.1016/j.bbamcr.2015.03.013. PubMed PMID: 25840011.

55. Schwarten M, Mohrluder J, Ma P, Stoldt M, Thielmann Y, Stangler T, et al. Nix directly binds to GABARAP: a possible crosstalk between apoptosis and autophagy. Autophagy. 2009;5(5):690-8. Epub 2009/04/14. doi: 10.4161/auto.5.5.8494. 
PubMed PMID: 19363302.

56. Chen ZH, Liu L, Cheng Q, Li YJ, Wu H, Zhang WL, et al. Mitochondrial E3 ligase MARCH5 regulates FUNDC1 to fine-tune hypoxic mitophagy. Embo Rep. 2017;18(3):495-509. doi: 10.15252/embr.201643309. PubMed PMID: WOS:000395061000017.

57. Wu S, Zhou F, Zhang Z, Xing D. Mitochondrial oxidative stress causes mitochondrial fragmentation via differential modulation of mitochondrial fission-fusion proteins. The FEBS journal. 2011;278(6):941-54.

58. Archer SL. Mitochondrial dynamics - mitochondrial fission and fusion in human diseases. New England Journal of Medicine. 2013;369(23):2236-51.

59. Yu T, Sheu SS, Robotham JL, Yoon Y. Mitochondrial fission mediates high glucose-induced cell death through elevated production of reactive oxygen species. Cardiovasc Res. 2008;79(2):341-51. Epub 2008/04/29. doi: 10.1093/cvr/cvn104. PubMed PMID: 18440987; PubMed Central PMCID: PMCPMC2646899.

60. Sheridan C, Martin SJ. Mitochondrial fission/fusion dynamics and apoptosis. Mitochondrion. 2010;10(6):640-8.

61. Many A, Hubel CA, Fisher SJ, Roberts JM, Zhou Y. Invasive cytotrophoblasts manifest evidence of oxidative stress in preeclampsia. The American journal of pathology. 2000;156(1):321-31.

62. Bainbridge SA, Roberts JM. Uric acid as a pathogenic factor in preeclampsia. Placenta. 2008;29:67-72.

63. Holland O, Nitert MD, Gallo LA, Vejzovic M, Fisher JJ, Perkins AV. Placental mitochondrial function and structure in gestational disorders. Placenta. 2017;54:2-9. 


\section{Supplementary information}

\section{Supplementary Figures}

To confirm whether mitochondria are a significant source for ROS in placentae upon hypoxia, the effect of the mitochondrially targeted antioxidant MitoQ and the systemically acting antioxidant quercetin on ROS formation and oxidative status was tested. Intracellular ROS levels increased more than two-fold in trophoblasts exposed for $24 \mathrm{~h}$ to hypoxia compared to normoxia-exposed trophoblasts (Supplementary Figure 1A). Moreover, the mitochondrially targeted antioxidant MitoQ (4 and $8 \mu \mathrm{M})$ ameliorated hypoxia-induced intracellular ROS formation in trophoblasts in a concentration-dependent manner. The systemically acting antioxidant quercetin $(3 \mu \mathrm{M})$, did not indiced significant reductions in intracellular ROS formation during exposure to hypoxia. Interestingly, the combination of the lowest concentration of MitoQ $(1 \mu \mathrm{M})$ and quercetin $(3 \mu \mathrm{M})$ significantly decreased intracellular ROS production upon $24 \mathrm{~h}$ exposure to hypoxia and was the only condition that was not significantly different from the normoxic condition (Supplementary Figure 1A). In agreement, the hypoxia-induced increase of the GSSG/GSH ratio, could be normalized by MitoQ ( 4 and $8 \mu \mathrm{M})$ and quercetin $(3 \mu \mathrm{M})$, but were still significantly different compared to the control condition (Supplementary Figure 1B). Interestingly, the combination of the lowest concentration of MitoQ $(1 \mu \mathrm{M})$ and quercetin $(3 \mu \mathrm{M})$, normalized the GSSG/ GSH ratio during exposure to hypoxia (Supplementary Figure 1B).

\section{Trophoblasts}
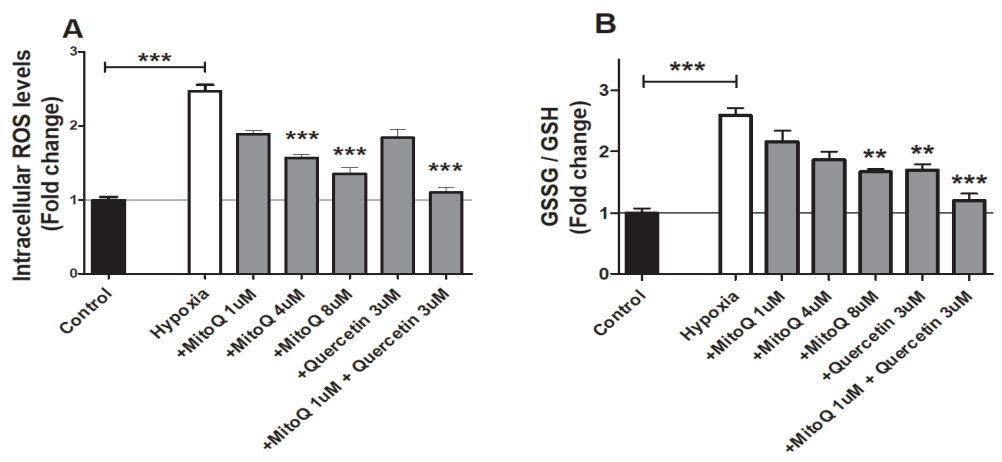

Supplementary Figure 1. Antioxidant intervention prevented hypoxia-induced oxidative stress in trophoblasts. Intracellular ROS levels using DCFH-DA (A) and GSSG/GSH ratio (B) were assessed in trophoblasts exposed to normoxia (control), hypoxia, hypoxia + MitoQ $(1,4$ or $8 \mu \mathrm{M})$, quercetin (3 $\mu \mathrm{M})$ or MitoQ $(1 \mu \mathrm{M})+$ quercetin $(3 \mu \mathrm{M})$ for $3 \mathrm{~h}$ (DCFH-DA-assay: $\mathrm{n}=6$-12/experimental condition $(\mathrm{n}=3$ experiments) and GSSG/GSH-assay: $\mathrm{n}=6,2$ and 2 /experimental condition ( $\mathrm{n}=3$ experiments)). Data are presented as fold change compared to the control and as mean with SEM. ${ }^{*} \mathrm{p} \leq 0.05, * * \mathrm{p} \leq$ 0.01 and ${ }^{* * *} \mathrm{p} \leq 0.001$. DCFH-DA: 2', 7'-Dichlorodihydrofluorescein diacetate and GSSG: Glutathione disulfide and GSH: Glutathione. 
While placentae exposed to hypoxia did not show alterations in proinflammatory and apoptotic-related mRNA transcripts, mRNA levels of tumor necrosis factor- $\alpha$ (TNF- $\alpha$ ), interleukin 6 (IL-6) and interleukin 8 (IL-8) as well as the pro-apoptotic ratio of Bcl-2associated X protein / anti-apoptotic B-cell lymphoma 2 (BAX/BCL-2), were significantly increased in trophoblasts exposed for $24 \mathrm{~h}$ to hypoxia compared to the control condition (Supplementary Figure 2C-D).
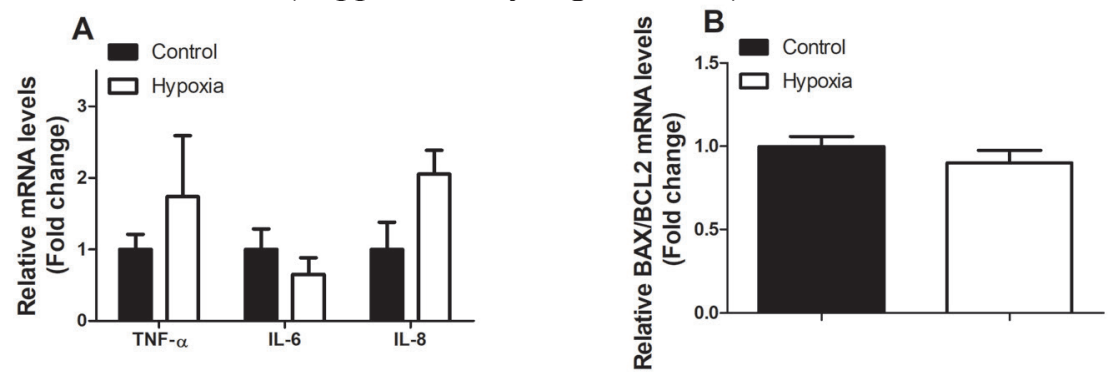

\section{Trophoblasts}
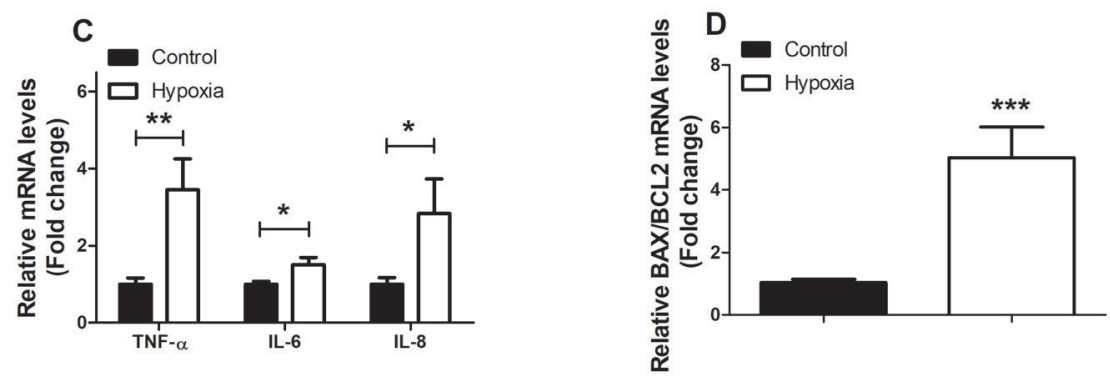

Supplementary Figure 2. Increased inflammation and apoptosis in trophoblasts upon hypoxia. mRNA transcript levels of TNF- $\alpha$, IL-6 and IL-8 (A) and ratio of BAX/BCL2 transcript levels (B) assessed in placental villous explants exposed to hypoxia or normoxia (control) for $3 \mathrm{~h}(\mathrm{n}=3)$ and mRNA transcript levels of TNF- $\alpha$, IL-6 and IL-8 (C) and ratio of BAX/BCL2 transcript levels (D) assessed in trophoblasts exposed to normoxia (control) or hypoxia for $24 \mathrm{~h}(\mathrm{n}=3-6 /$ experimental condition $(\mathrm{n}=3$ experiments)). Data are presented as fold change compared to the control and as mean with SEM. *p $\leq 0.05,{ }^{* *} \mathrm{p} \leq 0.01$ and $* * * \mathrm{p} \leq 0.001$. TNF- $\alpha$ : Tumor necrosis factor $\alpha$, IL-6: Interleukin 6, IL-8: Interleukin 8, BAX: pro-apoptotic Bcl-2-associated X protein and BCL: anti-apoptotic B-cell lymphoma 2.

In contrast to the increased reliance on glycolysis in placental cells upon hypoxia, no differences were observed in activity and mRNA expression levels of the rate-limiting enzyme HADH of the FAO pathway in both placental villous explants and trophoblasts exposed to hypoxia compared to the control group (Supplementary Figure 3A-D). 

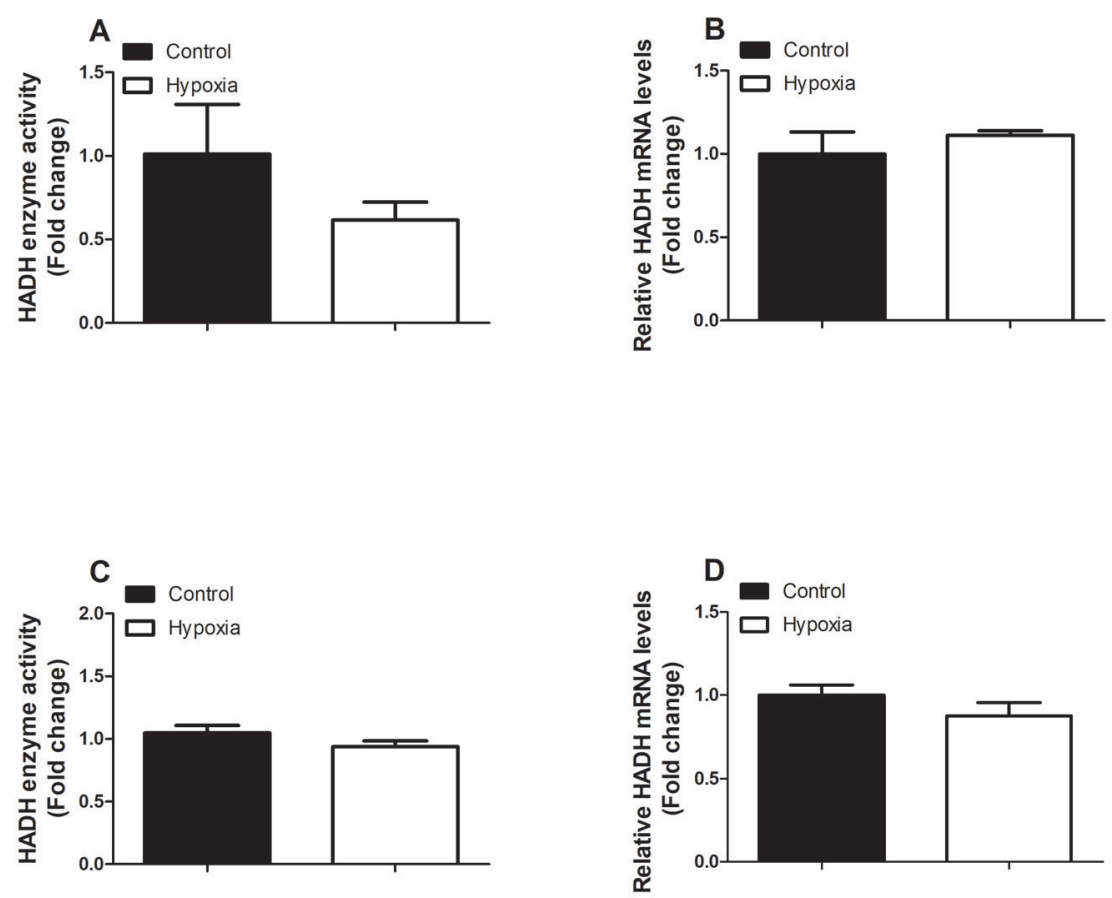

Supplementary Figure 3. Mitochondrial FAO unaltered in placental cells upon hypoxia. HADH enzyme activity (A) and mRNA transcript levels of HADH (B) assessed in placental villous explants exposed to hypoxia or normoxia (control) for $3 \mathrm{~h}(\mathrm{n}=3)$ and HADH enzyme activity $(\mathrm{C})$ and mRNA transcript levels of HADH (D) assessed in trophoblasts exposed to normoxia (control) or hypoxia for $24 \mathrm{~h}(\mathrm{n}=3,6$ and 6/experimental condition ( $\mathrm{n}=3$ experiments)). Data are presented as fold change compared to the control and as mean with SEM. HADH: 3-hydroxyacyl-CoA dehydrogenase.

Protein levels of nuclear-encoded sub-units of the ETC including Ndufb8, Sdhb, UQCR2 and ATP5A of respectively complex I, II, III and V, were not affected in placental villous explants exposed for $3 \mathrm{~h}$ to hypoxia (Supplementary Figure 4A). Also related mRNA transcript levels of Ndufb3, Cycl-1, COXIV and COXII of respectively complex I, III, IV and IV, were not affected in placental villous explants after exposure to hypoxia (Supplementary Figure 4B). Protein levels of UQCRC2 (complex III) as well as mRNA transcript levels of all investigated sub-units including mitochondrial encoded COXII were significantly decreased in trophoblasts upon 24h exposed to hypoxia (Supplementary Figure 4D-F). 
Protein levels of nuclear-encoded sub-units of the ETC including Ndufb8, Sdhb, UQCR2 and ATP5A of respectively complex I, II, III and V, were not affected in placental villous explants exposed for $3 \mathrm{~h}$ to hypoxia (Supplementary Figure 4A). Also related mRNA transcript levels of Ndufb3, Cycl-1, COXIV and COXII of respectively complex I, III, IV and IV, were not affected in placental villous explants after exposure to hypoxia (Supplementary Figure 4B). Protein levels of UQCRC2 (complex III) as well as mRNA transcript levels of all investigated sub-units including mitochondrial encoded COXII were significantly decreased in trophoblasts upon 24h exposed to hypoxia (Supplementary Figure 4D-F).

\section{Placental explants}
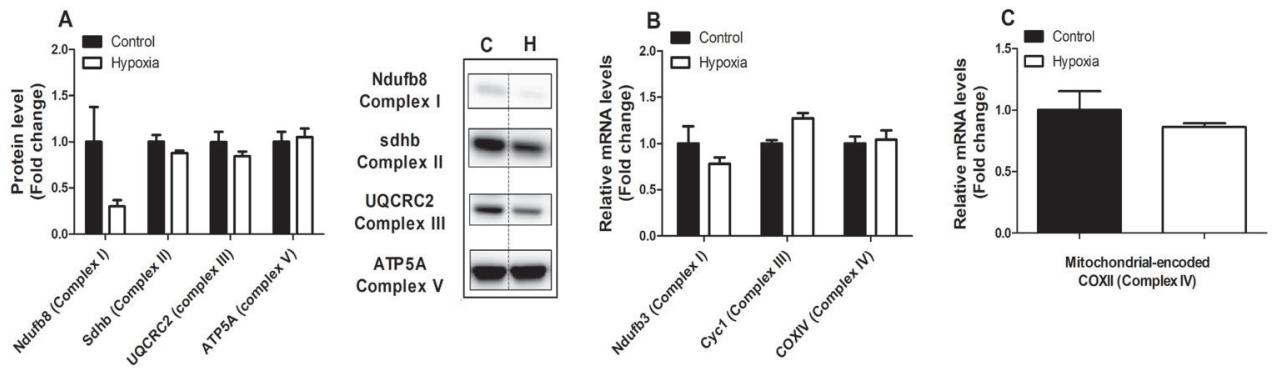

\section{Trophoblasts}
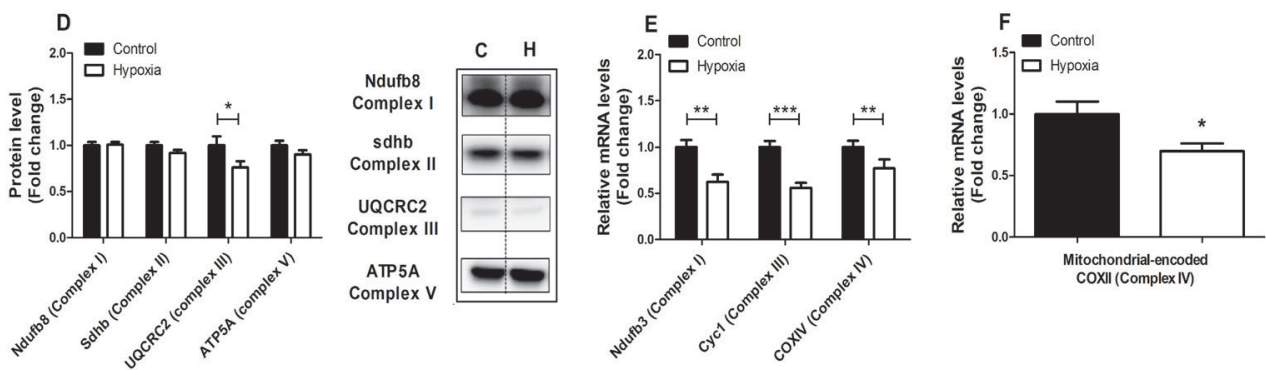

Supplementary Figure 4. Alterations in nuclear-encoded OXPHOS sub-units in trophoblasts upon hypoxia. Protein levels of nuclear-encoded OXPHOS sub-units (Ndufb8, Sdhb, UQCRC2 and ATP5A respectively Complex I, II, III and V) (A) and mRNA transcript levels of nuclear-encoded OXPHOS sub-units (Ndufb, Cycl-1, COXIV and COXII respectively Complex I, III, IV and IV) (B) assessed in placental villous explants exposed to normoxia (control) or hypoxia for $3 \mathrm{~h}(\mathrm{n}=3)$ and protein levels of nuclear-encoded OXPHOS sub-units (Ndufb8, Sdhb, UQCRC2 and ATP5A respectively Complex I, II, III and V) (C) and mRNA transcript levels of nuclear-encoded OXPHOS sub-units (Ndufb, Cycl-1, COXIV and COXII respectively Complex I, III, IV and IV) (D) assessed in trophoblasts exposed to normoxia (control) or hypoxia for $24 \mathrm{~h}(\mathrm{n}=3-6 /$ experimental condition $(\mathrm{n}=3$ experiments)). Representative immunoblots are shown and Western blots were corrected for total protein loading assessed by Ponceau S Staining with adjusted contrast equally applied to the whole photograph. Black boxes around the representative pictures indicate that they were cut from the same Western blot. Data are presented as fold change compared to the control and as mean with SEM. Ndufb3: NADH dehydrogenase [ubiquinone] 1 beta subcomplex subunit 3, Sdhb: Succinate dehydrogenase [ubiquinone] iron-sulfur subunit, UQCRC2: Cytochrome b-c1 complex subunit 2, ATP5A: ATP synthase F1 subunit alpha, Cyc1: Cytochrome C1, COXIV: Cytochrome c oxidase subunit IV and COXII: Cytochrome c oxidase subunit II. 
All investigated constituents of the autophagy in our study, were unaltered in placental villous explants exposed for $3 \mathrm{~h}$ to hypoxia compared to the control condition (Supplementary Figure 5A-B). In cultured trophoblasts, 24 h hypoxia resulted in a significant decrease in autophagy-associated LC3BI and LC3BII protein levels while the ratio of LC3BI/LC3BII was significantly increased compared to the normoxic condition. In addition, while both protein as well as mRNA levels of GABA Type A Receptor Associated Protein Like 1 (GABARAPL1) were increased in response to hypoxia, protein levels of SQSTM1 were decreased and its transcript levels increased upon hypoxia (Supplementary Figure 5C-D). OPTN mRNA levels increased 2-fold in response to hypoxia (Supplementary Figure 5D).

\section{Placental explants}
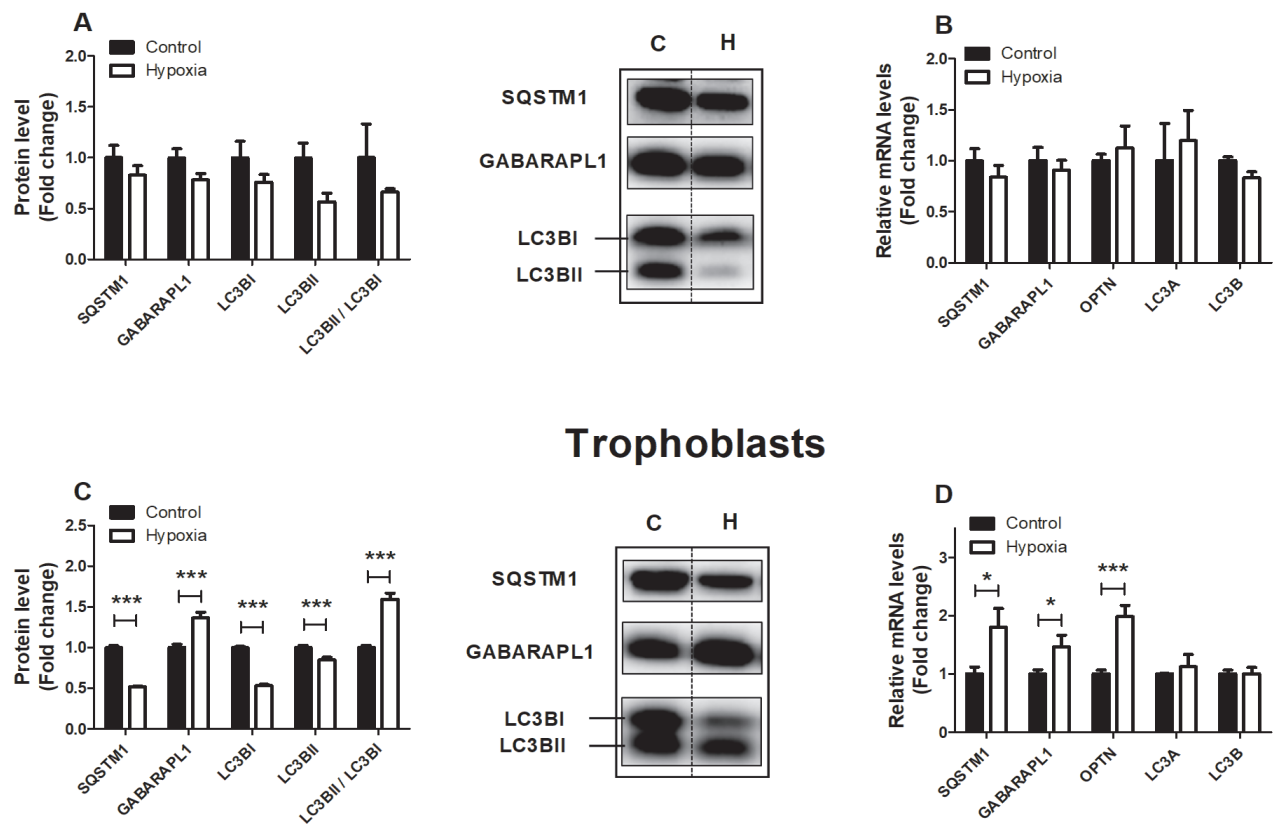

Supplementary Figure 5. Expression of key constituents of the autophagy machinery is altered in trophoblast upon hypoxia. Autophagy-associated protein levels of SQSTM1, GABARAPL1, LC3BI, LC3BII and LC3BII / LC3BI ratio (A) and mRNA transcript levels of SQSTM1, GABARAPL1, OPTN and LC3B (B) assessed in placental villous explants exposed to normoxia (control) or hypoxia for $3 \mathrm{~h}$ $(n=3)$ and autophagy-associated protein levels of SQSTM1, GABARAPL1, LC3BI and LC3BII (C) and mRNA transcript levels of SQSTM1, GABARAPL1, OPTN and MAP1LC3A/B (D) assessed in trophoblasts exposed to normoxia (control) or hypoxia for $24 \mathrm{~h}(\mathrm{n}=3-6 /$ experimental condition $(\mathrm{n}=$ 3 experiments)). Representative immunoblots are shown and Western blots were corrected for total protein loading assessed by Ponceau S Staining with adjusted contrast equally applied to the whole photograph. Black boxes around the representative pictures indicate that they were cut from the same Western blot. Data are presented as fold change compared to the control and as mean with SEM. * $\mathrm{p} \leq$ 0.05 and $* * * p \leq 0.001$. SQSTM1: Sequestosome 1, GABARAPL1: GABA Type A Receptor Associated Protein Like 1, LC3B: Microtubule-associated protein 1 light chain 3 beta, OPTN: Optineurin and LC3A: Microtubule-associated protein 1 light chain 3 alpha. 


\section{Supplementary Tables}

\section{Supplementary Table 1. Primers used for qPCR}

\begin{tabular}{|c|c|c|}
\hline Target & Sense primer $\left(5^{\prime}-3^{\prime}\right)$ & Antisense primer (3'-5') \\
\hline Catl & GATGTGCATGCAGGACAATCAG & GCTTCTCAGCATTGTACTTGTCC \\
\hline XO & AGAAAGTTGGGGCTGAGTGG & GCAGGCATTGGCAGAAAAGT \\
\hline SODI & GGTCCTCACTTTAATCCTCTAT & CATCTTTGTCAGCAGTCACATT \\
\hline MnSOD2 & TGGACAAACCTCAGCCCTAACG & \begin{tabular}{|l} 
TGATGGCTTCCAGCAACTCCC \\
\end{tabular} \\
\hline$T N F-\alpha$ & ATGCCTTTAGATGTGAGCTAACAGTAGGTA & CGTACAGCCATCAAAAAGGGACAC \\
\hline$B A X$ & GGTCTTTTTCCGAGTGGCAG & CACAGGGCCTTGAGCAC \\
\hline$B C L-2$ & GTCTTTTTCCGAGTGGCAGC & GTAGAAAAGGGCGACAACCC \\
\hline RPL13A & CCTGGAGGAGAAGAGGAAAGAGA & TTGAGGACCTCTGTGTATTTGTCAA \\
\hline COXII & ACCTGCGACTCCTTGACGTT & GGGGGCTTCAATCGGGAGTA \\
\hline CS & GATGTGTCAGATGAGAAGTTACGAGACT & TGGCCATAGCCTGGAACAA \\
\hline$H A D H$ & TGGCTTCCCGCCTTGTC & TGGAGCCGGTCCACTATCTTC \\
\hline HKII & $\begin{array}{l}\text { GTAAATACAGTGGATCTCAATCTTCGGG } \\
\end{array}$ & CAAGGATTTGAGATGATTCGCTATTCA \\
\hline GLUTI & TCTGGGCTGCCGGGTTCTAG & TTTGCAGGCTCCCACAGGC \\
\hline Cycll & GCATTCGGAGGGGTTTCCAG & CCGCATGAACATCTCCCCA \\
\hline COXIV & CCATGGATGAGAAAGTCGAGT & CGTTCGAGCCCCTGTTCA \\
\hline PGC-1 $\alpha$ & AAGCCACTACAGACACCGC & TCGTAGCTGTCATACCTGGG \\
\hline$P G C-1 \beta$ & CAGAACAAGGAGGCGGAGGTC & AGGTCCAAGTTTGCGAAGC \\
\hline NRFI & GCACCTTTGGAGAATGTGGT & CTGGGATAAATGCCCGAAG \\
\hline NRF2 & CTCACCTGGGAACAGAACAGGAA & ACCCAAGAAATGCAGTCTCGAGC \\
\hline$E R R a$ & TGCTGCTCACGCTACCGCTC & TCGAGCATCTCCAAGAACAGC \\
\hline Tfam & GAAAGATTCCAAGAAGCTAAGGGTGATT & TCCAGTTTTCCTTTACAGTCTTCAGCTTTT \\
\hline PPARa & CAGAACAAGGAGGCGGAGGTC & AGGTCCAAGTTTGCGAAGC \\
\hline PPARO & TGACCAAAAAGAAGGCCCGC & GTCGTGGATCACAAAGGGCG \\
\hline SQSTMI & GGTGCACCCCAATGTGATCT & CGCAGACGCTACACAAGTCG \\
\hline PINKI & GAAAGCCGCAGCTACCAAGA & AGCACATTTGCGGCTACTCG \\
\hline PARK2 & GGTTTGCCTTCTGCCGGGAATG & CTTTCATCGACTCTGTAGGCCTG \\
\hline FUNDCl & GAAACGAGCGAACAAAGCAG & GCAAAAAGCCTCCCACAAAT \\
\hline$B N I P 3$ & AGCGCCCGGGATGCA & CCCGTTCCCATTATTGCTGAA \\
\hline BNIP3L & CTGCGAGGAAAATGAGCAGTCTCT & GCCCCCCATTTTTCCCATTG \\
\hline OPTN & AAGGAGCAACTGGCATTGCA & TCTCCATCAAGGACTGCCTG \\
\hline GABARAPLI & ATCGGAAAAAGGAAGGAGAAAAGATC & CAGGCACCCTGGCTTTTGG \\
\hline$L C 3 A$ & CCTGGACAAGACCAAGTTTTTG & GTCTTTCTCCTGCTCGTAGATG \\
\hline$L C 3 B$ & ACCATGCCGTCGGAGAAGAC & TCTCGAATAAGTCGGACATCTTCTACTCT \\
\hline Fis-1 & CCTGGTGCGGAGCAAGTACAA & TCCTTGCTCCCTTTGGGCAG \\
\hline DNMIL & CGACTCATTAAATCATATTTTCTCATTGTCAG & TGCATTACTGCCTTTGGCACACT \\
\hline Mffnl & CTGAGGATGATTGTTAGCTCCACG & CAGGCGAGCAAAAGTGGTAGC \\
\hline Mffin 2 & TGGACCACCAAGGCCAAGGA & TCTCGCTGGCATGCTCCAC \\
\hline OPAI & TACCAAAGGCATTTTGTAGATTCTGAGTT & GCATGCGCTGTATACGCCAA \\
\hline IL-6 & CCTGAACCTTCCAAAGATGGC & CACCAGGCAAGTCTCCTCATT \\
\hline IL-8 & TTAGAACTATTAAAACAGCCAAAACTCCACA & CAAGTTTCAACCAGCAAGAAATTACTAATATTG \\
\hline Nduffo3 & ACAGACAGTGGAAAATTGAAGGG & GCCCATGTATCTCCAAGCCT \\
\hline
\end{tabular}

Cat1: Catalase-1, XO: Xanthine oxidase, SOD1: Superoxide dismutase 1, MnSOD2: Manganese-dependent superoxide dismutase, TNF- $\alpha$ : Tumornecrosefactor $\alpha$, BAX: Pro-apoptotic Bcl-2-associated X protein, BCL: Anti-apoptotic B-cell lymphoma 2, RPL13A: Ribosomal Protein L13a, COXII: Cyclo-oxygenase 2, CS: Citrate synthase, HADH: 3-hydroxyacyl-CoA dehydrogenase, HKII: Hexokinase, GLUT1: Glucose transporter 1, Cyc1: Cytochrome C1, COXIV: Cytochrome c oxidase subunit IV, PGC-1 $\alpha$ : Peroxisome proliferator-activated receptor gamma coactivator 1-alpha, PGC-1B: Peroxisome proliferator-activated receptor gamma coactivator 1-beta, NRF1: Nuclear respiratory factor 1, ERRa: Estrogen-related receptor alpha, Tfam: Transcription factor A, PPAR : Peroxisome proliferator-activated receptor alpha, PPARS: Peroxisome proliferator-activated receptor delta, SQSTM1: Sequestosome 1, PINK1: PTEN-induced kinase 1, PARK2: Parkin, FUNDC1: FUN14 domain containing 1, BNIP3: BCL2/ adenovirus E1B $19 \mathrm{kDa}$ protein-interacting protein 3, BNIP3L: BCL2/adenovirus E1B 19 kDa protein-interacting protein 3-like, OPTN: Optineurin, GABARAPL1: GABA Type A Receptor Associated Protein Like 1, LC3A: Microtubule-associated protein 1 light chain 3 alpha, LC3B: Microtubule-associated protein 1 light chain 3 beta, Fis-1: Fission 1 protein, DNM1L: Dynamin-related protein 1, Mfn 1: Mitofusin-1, Mfn2: Mitofusin-2, OPA1: Optic atrophy protein 1. IL-6: Interleukin 6, IL-8: Interleukin 8 and Ndufb3: NADH oxidoreductase subunit B3. 


\section{Supplementary Table 2. Antibodies used for western blot}

\begin{tabular}{|l|l|l|l|l|}
\hline Target & RRID & Company & Product number & Dilution \\
\hline HKII & AB_2232946 & Cell Signaling Technology & Cat\#2867 & $1: 1000$ \\
\hline OXPHOS & AB_2629281 & MitoScience LLC & Cat\# MS604 & $1: 1000$ \\
\hline PGC-1a & AB_10697773 & Millipore & Cat\# 516557 & $1: 1000$ \\
\hline NRF1 & AB_2154534 & Abcam & Cat\# ab55744 & $1: 1000$ \\
\hline ERRa & AB_1523580 & Abcam & Cat\# ab76228 & $1: 1000$ \\
\hline Tfam & AB_10682431 & Millipore & Cat\# DR1071 & $1: 1000$ \\
\hline SQSTM1 & AB_10624872 & Cell Signaling Technology & Cat\# 5114 & $1: 1000$ \\
\hline PINK1 & AB_10127658 & Novus Biologicals & Cat\# BC100-494 & $1: 2000$ \\
\hline PARK2 & AB_2159920 & Cell Signaling Technology & Cat\# 4211 & $1: 1000$ \\
\hline FUNDC1 & AB_10609242 & Santa Cruz Biotechnology & Cat\# sc-133597 & $1: 500$ \\
\hline BNIP3 & AB_2259284 & Cell Signaling Technology & Cat\# 3769S & $1: 1000$ \\
\hline BNIP3L & AB_2688036 & Cell Signaling Technology & Cat\# 12396 & $1: 1000$ \\
\hline GABARAPL1 & AB_2294415 & Proteintech Group & Cat\# 11010-1-AP & $1: 1000$ \\
\hline LC3B & AB_915950 & Cell Signaling Technology & Cat\# 2775 & $1: 1000$ \\
\hline DNM1L & AB_10950498 & Cell Signaling Technology & Cat\# 8570 & $1: 1000$ \\
\hline
\end{tabular}

HKII: Hexokinase II, OXPHOS: Oxidative phosphorylation, antibody cocktail (containing NADH: Ubiquinone oxidoreductase subunit B8 (NDUFB8), Succinate dehydrogenase complex, subunit B (SDHB), ubiquinol cytochrome c reductase core protein 2 (UQCRC2), Mitochondrially encoded cytochrome c oxidase I (mt-COI), ATP synthase, $H+$ transporting, mitochondrial F1 complex, alpha (ATP5A)), PGC-1 $\alpha$ : Proliferative activated receptor gamma, coactivator 1 alpha, NRF1: Nuclear respiratory factor 1, ERRa: Estrogen Related Receptor alpha, Tfam: Mitochondrial transcription factor A, SQSTM1: Sequestosome 1, PINK1: PTEN-induced kinase 1, PARK2: Parkin, FUNDC1: FUN14 domain-containing protein 1, BNIP3: BCL2/Adenovirus E1B $19 \mathrm{kDa}$ protein-interacting protein 3, GABARAPL1: $\gamma$-aminobutiric acid receptor-associated protein-like 1, LC3B: Microtubule associated protein 1A/1B-light chain 3 beta and DNM1L: Dynamin 1 Like. 


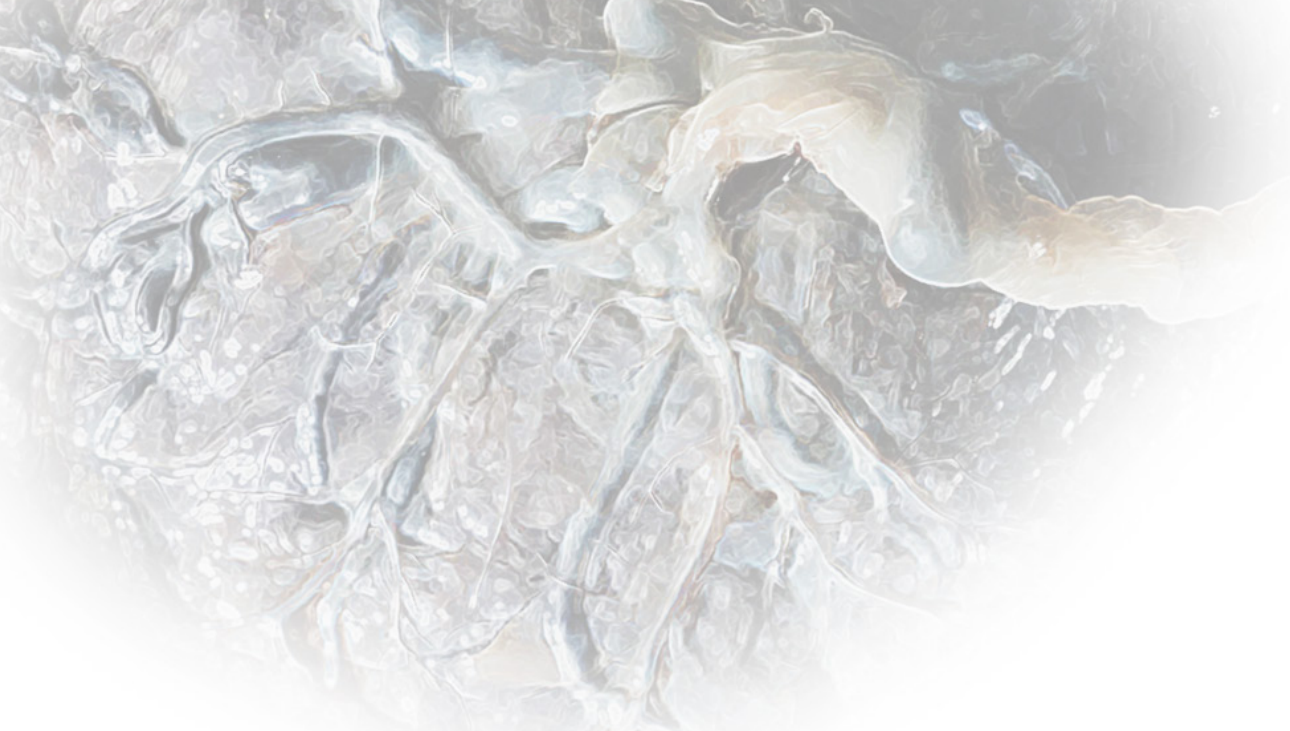




\section{Chapter 5}

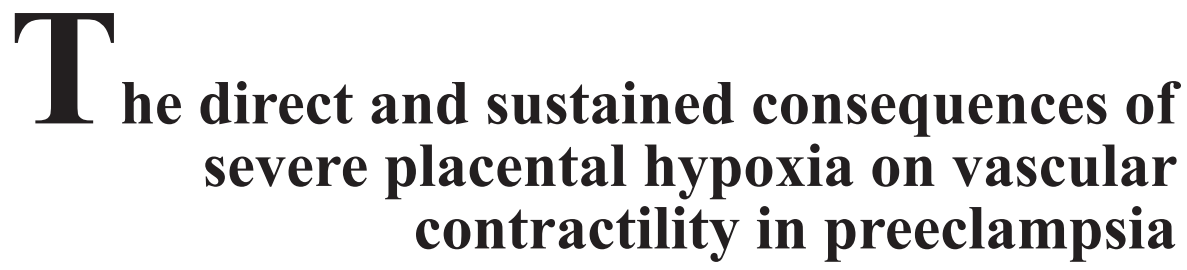

Philippe Vangrieken, Salwan Al-Nasiry, Ger M.J Janssen, Antje R. Weseler, Marc E. Spaanderman, Aalt Bast, Paul M.H. Schiffers

PLoS ONE 13(8): e0202648. 


\section{Abstract}

\section{Introduction}

Preeclampsia is a major health problem in human pregnancy, severely complicating $5-8 \%$ of all pregnancies. The emerging molecular mechanism is that conditions like hypoxic stress trigger the release of placental messengers into the maternal circulation, which causes preeclampsia. Our objective was to develop an in vitro model, which can be used to further elucidate the molecular mechanisms of preeclampsia and which might be used to find a remedy.

\section{Methods}

Human non-complicated term placentae were collected. Placental explants were subjected to severe hypoxia and the conditioned media were added to chorionic arteries that were mounted into a myograph. Contractile responses of the conditioned media were determined, as well as effects on thromboxane-A2 (U46619) induced contractility. To identify the vasoactive compounds present in the conditioned media, specific receptor antagonists were evaluated.

\section{Results}

Factors released by placental explants generated under severe hypoxia induced an increased vasoconstriction and vascular contractility to thromboxane-A2. It was found that agonists for the angiotensin-I and endothelin-1 receptor released by placental tissue under severe hypoxia provoke vasoconstriction. The dietary antioxidant quercetin could partially prevent the acute and sustained vascular effects in a concentration-dependent manner.

\section{Conclusion}

Both the acute vasoconstriction, as well as the increased contractility to U46619 are in line with the clinical vascular complications observed in preeclampsia. Data obtained with quercetin supports that our model opens avenues for e.g. nutritional interventions aimed at treating or preventing preeclampsia. 


\section{Introduction}

Preeclampsia (PE) is the most frequently encountered severe complication during pregnancy with a clinical spectrum ranging from relatively mild to life-threatening $(1,2)$. It is not simply de novo onset of hypertension and proteinuria, but rather a syndrome involving multiple organs, which results in end-organ dysfunction (3).

The etiology of PE is enigmatic and except for delivery no treatment exists, which is associated with iatrogenic prematurity and lifelong disabilities for mother and child $(4,5)$. Prevention and solid prediction are still not possible, and symptomatic clinical management is mainly directed at preventing maternal morbidity and mortality (3).

The leading hypothesis considers disturbed placental development during the first trimester of pregnancy to be the main cause (3). Impaired remodelling of maternal spiral arteries is considered to be crucial in the onset of PE. During PE there is an impaired invasion of trophoblast cells into the inner third of the myometrium (6, 7). As a consequence, no trophoblastic plugs are formed in the ends of the spiral arteries, which is associated with early perfusion of the placenta in de first trimester of pregnancy and impaired remodelling of the spiral arteries in the second and third trimester $(8,9)$. Consequently, maternal blood enters the intervillous space at greater velocity creating relatively high fluctuations in placental perfusion and subsequently to placental hypoxia and reoxygenation $(8,10)$.

Evidence is accumulating, that oxidative stress by excessive production of ROS in $\mathrm{PE}$ increases trophoblast turnover, resulting in an accelerated differentiation of trophoblast cells followed by the release of specific placenta-secreted messengers (PSMs) into the maternal circulation that induce endothelial dysfunction, vasoconstriction, increased blood coagulation and inflammation (11-16). The exact nature of the PSMs and their specific interaction with maternal cells is still enigmatic.

As Davage et al. discussed, PE is unlikely the result of a single factor/molecular pathway. It involves various circulating factors acting on the maternal vascular wall that disturb the balance between vasodilatory and vasoconstrictor mechanisms (17). The diversity in the nature of potential instigators and their sources in PE, as well as the complex interactions with maternal targets, underlines the need for further investigation of the common converging pathways that link placental dysfunction with systemic maternal vascular pathology (17). 
Redman et al. showed that PE is not only an endothelial disease but also a disorder where the inflammatory changes of a normal pregnancy are exaggerated. These changes involve the endothelium, but also other components of the inflammatory network, including inflammatory leukocytes. This resulting systemic inflammation is not only associated with changes of the acute phase response like fever, increased CRP and angiotensinogen levels, but also with metabolic responses such as insulin resistance $(16,18)$. Furthermore, a list of bioactive circulating factors released by syncytiotrophoblasts, which are altered in PE was presented (16). This list includes corticotrophin-releasing hormone (CRH) that is known to have both peripheral and central actions. Its peripheral actions are mediated by the $\mathrm{CRH}-\mathrm{R} 1 / 2$ receptors, both expressed on the endothelium. The CRH-R2 is known to stimulate endothelium release of endothelin-1 (ET-1), a pro-inflammatory factor, which is also known to be involved in the genesis of preeclamptic hypertension $(16,19)$. Besides changes in the ET-1 signaling pathways, alterations in vascular angiotensin signaling pathways have been observed in many models of many vascular beds and have previously been linked to preeclamptic hypertension (5).

The major ethical barrier for studying the pathophysiology of PE and testing therapeutic interventions in pregnant woman underline the need for a human in vitro model for PE $(20,21)$.

This prompted us to develop an in vitro model for PE, which was used to subject placental explants to severe hypoxia to mimic the stress condition in PE and to collect PSMs. Furthermore, the acute and sustained vascular functional potencies of PSMs generated under severe hypoxia were determined on chorionic arteries since these arteries do not have an autonomic nerve supply and are depending on the local release of vasoactive compounds, which in fact permits investigation of direct actions of PSMs. To identify the vasoactive compounds in PSMs, specific receptor antagonists were tested. The food derived quercetin was tested in our in vitro model of PE to determine whether a dietary intervention could prevent a disturbed release of PSMs in response to hypoxic stress (22). 


\section{Methods}

\section{Patient characteristics}

Human term placentae ( $>38$ weeks gestational age) were collected from normal vaginal or caesarean deliveries performed by the Department of Obstetrics, Gynaecology at the Academic Hospital of Maastricht (azM). For this study, a total of 52 placentae were used within one hour after delivery. All experiments were approved by the Medical Ethics Committee Academic Hospital Maastricht and Maastricht University (METC 16-4-047). A total of 52 women were included in the study.

\section{Chemicals}

Acetylcholine (ACh), bradykinin (BK), sodium nitroprusside (SNP), carbachol (Car), angiotensin II (AngII), endothelin 1 (ET-1), serotonin (5-HT, 5-hydroxytriptamine), losartan (Los), bosentan (Bos) and ketanserin (Ket) (Sigma Chemical Co., St Louis, MO, USA). U46619, a thromboxane-A2 (TXA2) agonist analogue (Enzo Life sciences, USA). HEPES buffer containing (in $\mathrm{mM}$ ): $\mathrm{NaCl} 143.3, \mathrm{KCl}$ 4.7, $\mathrm{MgSO}_{4} 1.2, \mathrm{KH}_{2} \mathrm{PO}_{4} 1.2, \mathrm{CaCl}_{2} 2.5$, glucose 5.5 and HEPES $15(\mathrm{pH}=7.4)$. Krebs Ringer bicarbonate buffer (KRB) containing (in $\mathrm{mM}$ ): $\mathrm{NaCl} 118.3, \mathrm{KCl} 4.7, \mathrm{CaCl}_{2}$ 2.5, $\mathrm{MgSO}_{4} 1.2, \mathrm{KH}_{2} \mathrm{PO}_{4} 1.2, \mathrm{NaHCO}_{3} 25.0$ and glucose $11.1(\mathrm{pH}=7.4)$.

\section{Villous explant isolation and PSMs collection}

Three placentae from non-complicated caesarean deliveries were processed directly after delivery. Specimens were collected from the central region of the placentae at the maternal side. The basal plate of the specimens was removed and the remaining tissue was rinsed in an HEPES solution. Two times $30 \mathrm{~g}$ minced tissue of each placenta was transferred into $200 \mathrm{ml}$ pre-warmed $\left(37^{\circ} \mathrm{C}\right)$ HEPES buffer. This ratio was based on reference values for total maternal blood volume and placental weight (23, 24). Subsequently, 6 bottles were placed in a water bath at $37^{\circ} \mathrm{C}$ for 3 hours were 3 bottles were constantly aerated with room air $\left(21 \% \mathrm{O}_{2}\right)$ as standard culture condition and three bottles at $100 \% \mathrm{~N}_{2}$ to expose the placental cells to severe hypoxia (Figure 1A). After these exposures, the solutions were centrifuged at $500 \mathrm{~g}$ for 10 minutes at $4{ }^{\circ} \mathrm{C}$. The resulting conditioned medium was stored in aliquots of $3 \mathrm{ml}$ at $-80{ }^{\circ} \mathrm{C}$ until use. 
A

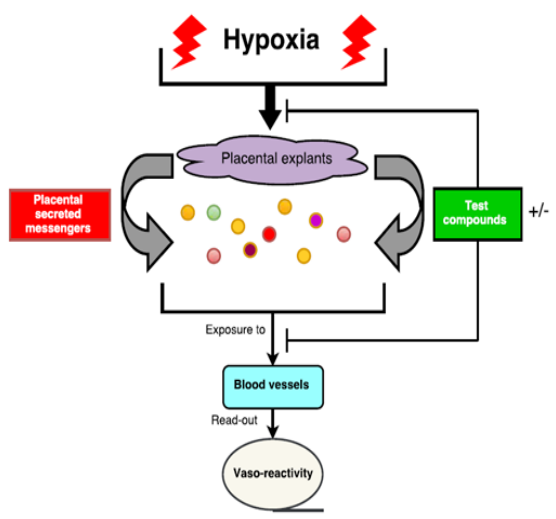

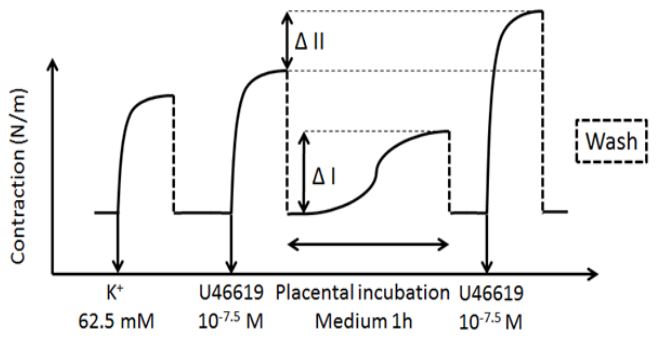

Figure 1. In vitro model for PE and testing the vascular effect of PSMs. Schematic overview of the In vitro model for PE where placental explants are exposed to hypoxia and PSMs are tested on chorionic arteries. Differences in contractile response are used as read-out (A) and a schematic overview for measuring the acute $(\Delta \mathrm{I})$ and sustained $(\Delta \mathrm{II})$ vascular effect of PSMs $(\mathrm{B})$.

\section{Chorionic artery isolation and mounting into a myograph}

Placental areas from the chorion plate containing chorionic arteries were macroscopically selected and excised from both vaginal and caesarean deliveries. The specimen was immediately immersed in HEPES solution $\left(4^{\circ} \mathrm{C}\right)$ and pinned in a Sylgaard dish with the foetal side orientated upwards. Second and third order branches of the chorionic arteries were dissected. Arterial segments of $2 \mathrm{~mm}$ were mounted in a myograph for recording of isometric tension development (DMT, Aarhus, Denmark). The organ chamber was filled with HEPES-buffer that was maintained at $37^{\circ} \mathrm{C}$. All arterial preparations were stretched to the diameter at which their individual mechanical performance was maximal. To achieve this, the arterial diameter was stepwise increased and the preparations were intermittently exposed to $65.5 \mathrm{mM}$ potassium-HEPES until maximal contractile responses were obtained. The value of these $\mathrm{KCl}$-contractions was presented as $\mathrm{K}+65.5 \mathrm{mM}$. The 175 blood vessel segments used for this study had a diameter of $549 \pm 61 \mu \mathrm{m}$.

\section{Functional characterization of chorionic arteries}

For the characterisation of the chorionic arteries, the following drugs were used: for vasodilatation $\mathrm{ACh}, \mathrm{BK}, \mathrm{SNP}$ and Car and for vasoconstriction: AngII, ET-1, 5-HT and U46619. Drug solutions were prepared daily and protected from light until use. All drugs were dissolved and diluted in miliQ, except for U46619, which was dissolved in ethanol absolute. Relaxation responses were expressed as a percentage of the maximal contraction achieved by either $65.5 \mathrm{mM} \mathrm{KCl}$ or $30 \mathrm{nM} \mathrm{U} 46619$. 


\section{Tissue viability}

Placental tissue used for the PSM collection was evaluated for viability for both the standard culture $\left(21 \% \mathrm{O}_{2}\right)$ and the severe hypoxic condition by studying lactate dehydrogenase (LDH) activity using cytotoxicity detection kit (Cat. No.11644793001, Roche, Mannheim, Germany). LDH levels, which are indicative of cell death were measured in the conditioned medium from both conditions for all three placentae.

\section{Vasoactive responses to PSMs}

Chorionic arteries were first pre-contracted by U46619 (30 nM). After washing and complete relaxation ( \pm 30 minutes), $3 \mathrm{ml}$ of the thawed conditioned media containing PSMs of either the standard culture $\left(21 \% \mathrm{O}_{2}\right)$ or the severe hypoxic condition, was added to the organ bath containing $4 \mathrm{ml}$ of HEPES buffer. After 1 hour the PSM induced vasocontraction was measured, followed by a washing step (Figure 1B, I). After complete relaxation ( \pm 30 minutes), the blood vessels were pre-contracted again by U46619 (30 nM). Differences between the vascular contractile response of the 2nd and 1 st pre-contraction were calculated (Figure 1B, II).

\section{Characterisation of vasoactive PSMs released under hypoxia}

The vascular contractile response induced by conditioned media containing PSMs released under severe hypoxia was tested when the blood vessels were pre-incubated with the following antagonists: $30 \mu \mathrm{M}$ Los, $30 \mu \mathrm{M}$ Bos, $30 \mu \mathrm{M}$ Ket (AT-1, ET-1 and 5-HT2-receptor blockers) or $30 \mu \mathrm{M}$ Bos + Los.

\section{Effects of quercetin on the release of PSMs under hypoxia}

At the start of the PSMs generation procedure, quercetin $(0.3,1,3,10$ and $20 \mu \mathrm{M})$ was added to $200 \mathrm{ml}$ pre-warmed HEPES buffer $\left(37^{\circ} \mathrm{C}\right)$ containing $30 \mathrm{~g}$ placental tissue. This was again followed by 3 hours $100 \% \mathrm{~N}_{2}$ aeration. Differences in vascular contractile response to these generated PSMs as well as changes in sustained responsiveness to U46619 were tested.

\section{Data analysis}

Results are expressed as mean \pm SEM and $n$ refers to the number of placentae from which blood vessels were isolated. Contractile responses are expressed as a percentage of the maximal contractile response to $\mathrm{KCl} 65.5 \mathrm{mM}$. By using GraphPad Software inc. La Jolla, CA, USA), the maximal contractile response (Emax) and the logarithm of the half maximal effective concentration (pEC50) of various compounds were calculated. For the viability test, a nonparametric Mann-Whitney test was used. For the analysis of the vascular contractile effect to PSMs, ET-1, or AngII and the characterisation of the placental vessels, a two-way ANOVA followed by a 
Bonferroni posthoc test and a nonparametric Mann-Whitney test were used. To determine the consistency of the of PSMs and the effect of quercetin on the release of PSMs under hypoxia, a two-way ANOVA followed by a Bonferroni posthoc test and a nonparametric Kruskal-Wallis test followed by a Dunn's multiple comparison test were used. A p-value $<0.05$ was considered as significant difference and presented as follows: Ns: $\mathrm{P}>0.05, * \mathrm{P} \leq 0.05, * * \mathrm{P} \leq 0.01, * * * \mathrm{P} \leq 0.001$. 


\section{Results}

\section{Functional characterisation chorionic arteries}

\section{Vasodilation}

The NO donor sodium nitroprusside (SNP, $30 \mu \mathrm{M}$ ) induced vasorelaxation up to 76 $\pm 23 \%$, indicating that chorionic arteries are NO-sensitive. No clear vascular relaxation was induced by $\mathrm{ACh}, \mathrm{BK}$ or Car after pre-contraction $(65.5 \mathrm{mM} \mathrm{KCl})$. However, after contraction by U46619 (30 nM), BK $(3 \mu \mathrm{M})$ induced vasodilation up to $40 \pm 20 \%$. The results indicate that Ach, BK or Car are not able to activate eNOS to induce a relevant NO-dependent relaxation. A pEC50 could be calculated from the concentration-dependent relaxation curves for Ach (7.9 \pm 0.8$), \operatorname{SNP}(6.7 \pm 0.5)$ and BK $(7.2 \pm 1.1)$ pre-contracted by U46619 (30 nM) (Figure 2).
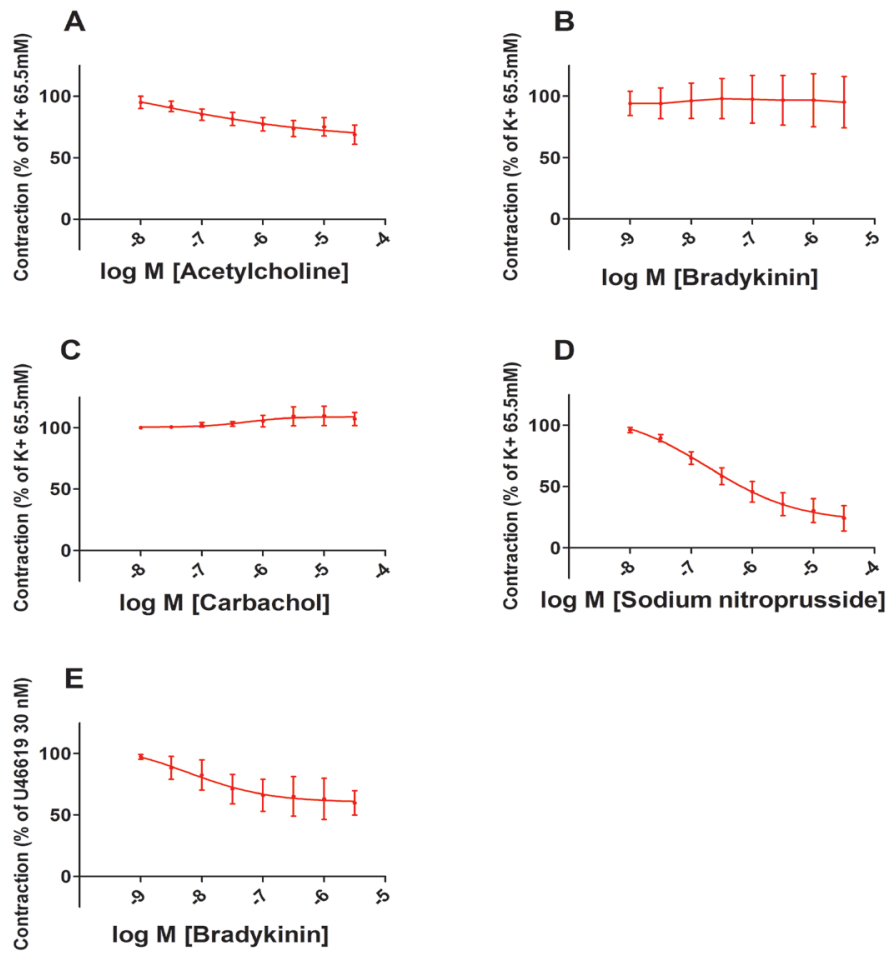

Figure 2. Vasodilation of chorionic arteries. Concentration-dependent relaxation of placental chorionic arteries induced by ACh (n=6) (A), BK (n=3) (B), Car $(n=3)(C)$ or SNP $(n=5)(D)$, after a pre-contraction induced by $\mathrm{KCl} 65.5 \mathrm{mM}$, except for $\mathrm{BK}$, which was after a pre-contraction by $\mathrm{U} 46619$ $(n=4)(E)$. 


\section{Vasoconstriction}

Dose-dependent vasoconstriction of chorionic arteries was tested for U46619, AngII, ET-1, 5-HT and BK. The maximal response related to the $65.5 \mathrm{mM} \mathrm{KCl}$ induced contraction was: $183 \pm 71,54 \pm 17,45 \pm 8$ and $66 \pm 20 \%$ respectively. BK ( $1 \mathrm{nM}-3$ $\mu \mathrm{M}, \mathrm{n}=3$ ), had no effect on baseline wall-tension. A pD2 could be calculated from the concentration-dependent relaxation curves for U46619 (7.9 \pm 0.4$)$, AngII (7.8 \pm $0.7)$, ET (6.2 \pm 0.9$), 5$-HT (6.9 \pm 0.4$)$ (Figure 3).
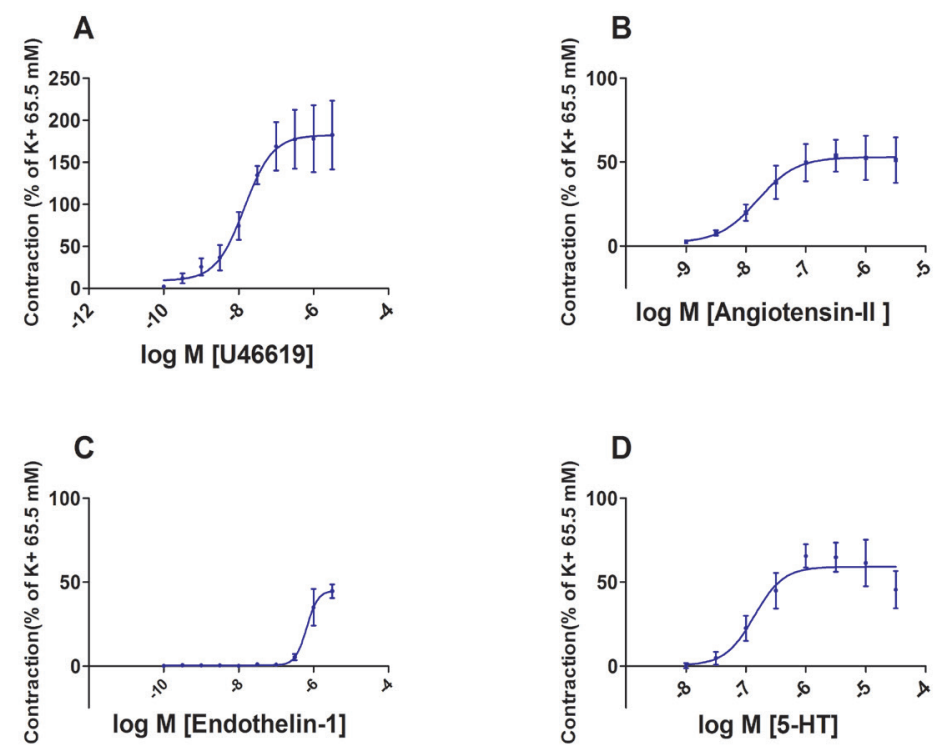

Figure 3. Vasoconstriction of chorionic arteries. Concentration-dependent contractions of placental chorionic arteries induced by U46619 ( $n=3)(A)$, AngII ( $n=3)(B)$, ET-1 $(n=4)(C)$, or 5-HT (n=6) (D), compared to a pre-contraction induced by $\mathrm{KCl} 65.5 \mathrm{mM}$.

\section{Tissue viability}

LDH levels of the conditioned medium of both the standard culture $\left(21 \% \mathrm{O}_{2}\right)$ and the severe hypoxic condition were compared. No significant difference $(p=0.1000)$ was found for LDH levels in the conditioned medium between the two conditions. This indicates that tissue viability was comparable between the two conditions after the 3 hours PSM collection procedure.

\section{Vasoactive responses of chorionic arteries to PSMS}

PSMs released under severe hypoxia resulted in a significant $(p=0.0002)$ time-dependent increased contraction of $78 \pm 33 \%$ compared to the standard culture condition $(20 \pm 9 \%)(n=8)$ after 1 hour (Figure 4A/B). To test the sustained effects on 
vascular contractility to U46619, the contraction induced by U46619 (30 nM) before and after exposure to PSMs was determined (Figure 4C). PSMs released under severe hypoxic conditions induced an increased response to U46619 (64 $\pm 30 \%$ ) compared to those produced by standard culture $\left(21 \% \mathrm{O}_{2}\right)$ conditions $(2 \pm 8 \%$; $=$ 0.0002) (Figure 4).
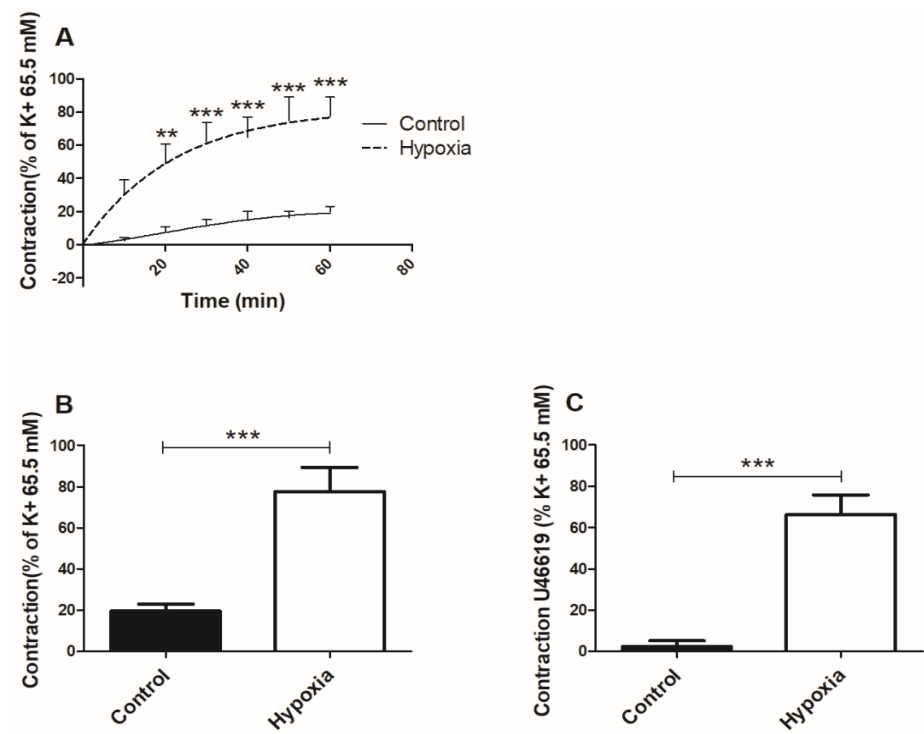

Figure 4. Vasoactive responses of chorionic arteries to PSMs. The vascular contractile response over time (60 min) induced by PSMs released during exposure to room air (control) $(n=8)$ or hypoxia $(n=8)$ (A). The maximum response after $60 \mathrm{~min}(\mathrm{n}=8)(\mathrm{B})$ and differences in vascular contractility to U46619 before and after exposure to PSMs for both conditions $(n=8)(C)$.

\section{Characterisation of vasoactive PSMs released under hypoxia}

PSMs released under severe hypoxia were added to chorionic arteries for 1 hour after a 15 min pre-incubation with the following antagonists: Bos, Los, Ket or Bos + Los. A $72 \pm 1 \%$ decrease $(p=0.0121)$ in vascular contractile response to PSMs was evoked when vessels were pre-incubated with Bos $(30 \mu \mathrm{M})$, a competitive ET-1 (A/B) receptor antagonist (Figure 5A and E). Los $(30 \mu \mathrm{M})$, a competitive AT-1 receptor antagonist decreased $(\mathrm{p}=0.0242)$ the PSMs induced contraction by $61 \pm 8 \%$ (Figure 5B and E). The combination losartan and bosentan $(30 \mu \mathrm{M})$ showed a decrease $(p=0.0121)$ of $87 \pm 2 \%$ (Figure $5 D$ and $E$ ). The contractile response was not affected $(\mathrm{p}=0.9212)$ by Ket $(30 \mu \mathrm{M})$, a competitive serotonin $(5-\mathrm{HT} 2)$ antagonist (Figure 5C and E). 

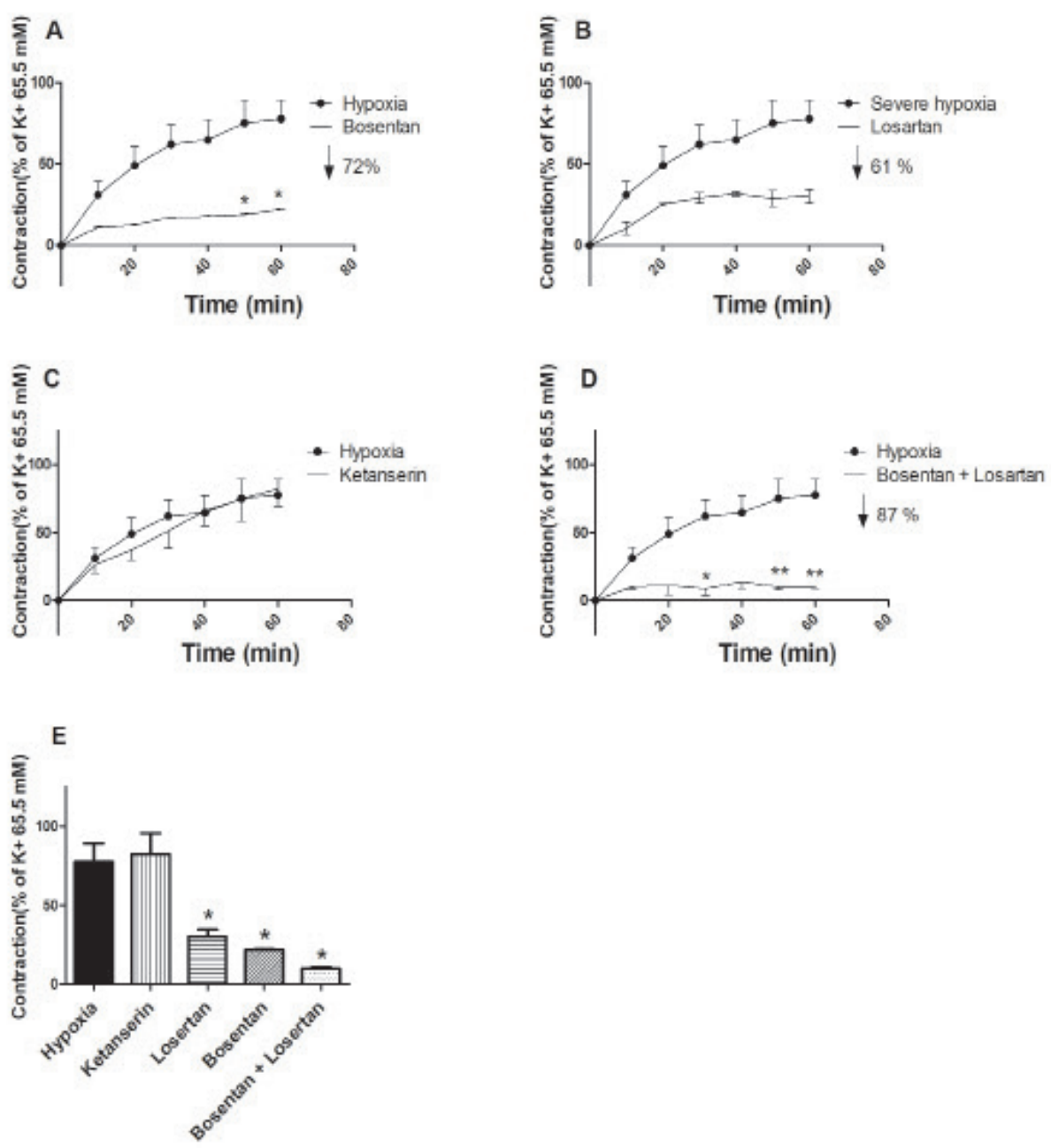

Figure 5. Characterization of vasoactive PSMs released under hypoxia. The vascular contractile response over time $(60 \mathrm{~min})$ induced by PSMs released under hypoxia in combination with a $15 \mathrm{~min}$ pre-incubation with either $30 \mu \mathrm{M}$ Bos (n=3) (A), or $30 \mu \mathrm{M} \operatorname{Ket}(n=3)(B)$, or $30 \mu \mathrm{M} \operatorname{Los}(n=3)(C)$, or $30 \mu \mathrm{M}$ Bos $+\operatorname{Los}(\mathrm{n}=3)(\mathrm{D})$. The maximum response to PSMs released under hypoxia after $60 \mathrm{~min}$ $(n=3)(E)$.

\section{Vascular contractile effects of ET-1 and AngII in isolation}

AngII $(3 \mu \mathrm{M})$, ET-I $(3 \mu \mathrm{M})$ or the combination, induced vasoconstriction of $53 \pm 19$, $45 \pm 8$ and $112 \pm 31 \%$ respectively, related to the contractile response of $65.5 \mathrm{mM}$ $\mathrm{KCl}$. The combination of ET-1 and AngII induced more vasoconstriction compared to the components in isolation, indicating that the pathways activated, are partially independent $(\mathrm{n}=4)$ (data not shown). 


\section{Consistency of the extraction procedure for PSMs}

To test the reproducibility of our experimental setting the coefficient of the variance within one and between three extraction procedures was determined. PSMs released under severe hypoxia from one placenta, induced a similar vascular contraction in chorionic arteries isolated from 3 different placentae $(\mathrm{CV}=4.5 \%)$. PSMs released under severe hypoxia form 3 different placentae also resulted in a similar $(\mathrm{CV}=$ $0.5 \%$ ) vascular contraction in chorionic arteries isolated from three different placentae.

\section{Effects of quercetin on the release of PSMs under hypoxia}

Quercetin $(0.3,1,3,10$ or $20 \mu \mathrm{M})$ added to $200 \mathrm{ml}$ pre-warmed HEPES buffer $\left(37^{\circ} \mathrm{C}\right)$ containing $30 \mathrm{~g}$ placental tissue followed by 3 hours $100 \% \mathrm{~N}_{2}$ aeration resulted in a concentration-dependent decreased vasoconstriction of $4 \pm 28,43 \pm 25,58 \pm 29,73$ \pm 25 and $91 \pm 12 \%$ respectively induced by the released PSMs (Figure 6A). Furthermore, a concentration-dependent decreased reactivity to $\mathrm{U} 46619$ of $23 \pm 12,30 \pm 15$, $36 \pm 9,52 \pm 7$ and $61 \pm 17 \%$ was found (Figure 6B).
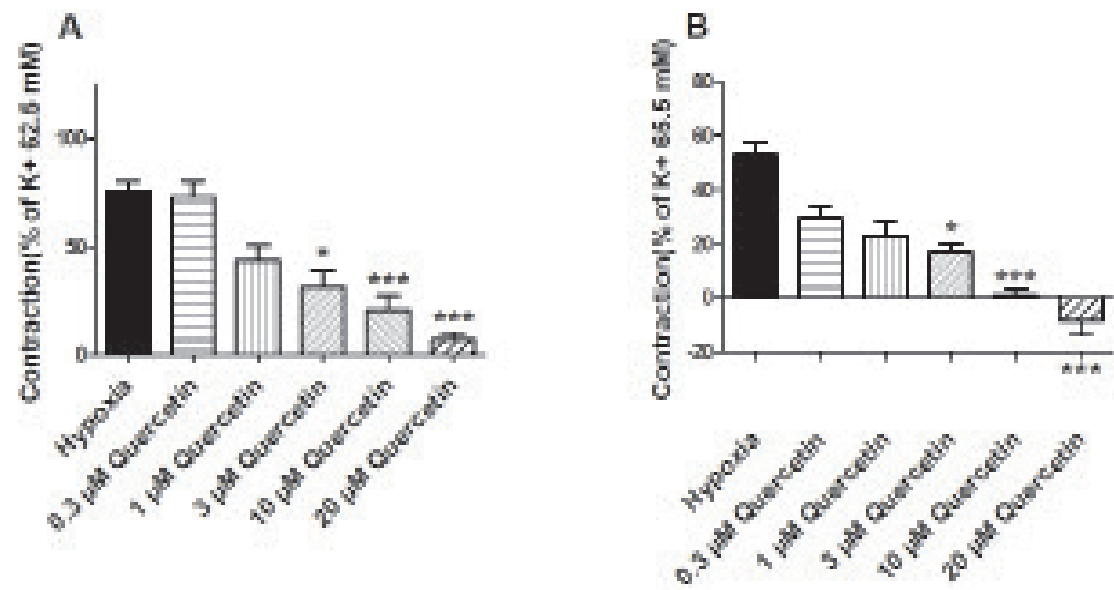

Figure 6. Effects of quercetin on the release of PSMs under hypoxia. The maximum vascular contractile response after $60 \mathrm{~min}$ induced by PSMs released under hypoxia (control) ( $\mathrm{n}=27)$, hypoxia in combination with $0.3,1,3,10$ or $20 \mu \mathrm{M}$ quercetin $(\mathrm{n}=8)(\mathrm{A})$ and the difference in vascular contractility to U46619 before and after exposure to the PSMs for each condition (B). 


\section{Discussion}

In this study, an in vitro model is presented for PE using placental villous explants of non-complicated term placentae and chorionic arteries. This model can be used to study the production of PSMs by the placenta that induce the acute vasoconstriction and sustained increased contractility observed in PE.

The vascular reactivity of chorionic arteries was characterised using various vasoactive compounds. Car was used to confirm that the absence of ACh-induced vasorelaxation was not due to the breakdown of ACh by cholinesterase. It was found that BK only induces vasorelaxation after a pre-contraction by U46619 (-40 $\pm 20 \%)$ and not after a pre-contraction by $\mathrm{KCl}$, which is known to inhibit the release of endothelium-derived hyperpolarizing factor $(\mathrm{EDHF})(25,26)$. It was therefore suggested that the vasorelaxation was induced by the released EDHF in response to BK (27). Since BK did not induced vasoconstriction as previously observed in umbilical arteries, it was concluded that no TXA2 was produced in response to BK, which could have masked the BK-induced vasorelaxation $(28,29)$. The endothelial independent vasorelaxator SNP induced a relaxation up to $76 \pm 23 \%$ in $\mathrm{KCl}$ pre-contracted arteria and was thereby the most potent vasorelaxator. Even though Ach and BK did not induce a NO-dependent vasorelaxation, SNP proved that the vessels used for our further experiments are NO-sensitive. Concentration-dependent vasoconstrictions were induced by U46619, AngII, ET-1, 5-HT with a maximal response related to the contractile response of $65.5 \mathrm{mM} \mathrm{KCl}$ of $183 \pm 71,54 \pm 17,45 \pm 8$ and $66 \pm 20 \%$ respectively. This shows that arteries contained functional active AT-1, ET-1 and 5-HT2 receptors.

In our in vitro model, we found that PSMs released under severe hypoxia induced a $58 \%$ increase in vascular contraction compared to the PSMs obtained from the standard culture $\left(21 \% \mathrm{O}_{2}\right)$ condition. Beside altered vasoconstriction, the PSMs released under severe hypoxia also caused a $64 \%$ sustained increase in vascular contractility to U46619. With the use of specific receptor antagonists, the AT-1 and ET-1 receptors were identified as the main contributors to the direct vasoconstrictive activity of the PSMs. These results obtained in our model are in line with the paradigm that stress factors, like hypoxia, in placental tissue lead to the release of PSMs causing acute high blood pressure and increased risk for high blood pressure in later life of the mother (5).

Khalil et al. already suggested that bioactive factors like AT-1-agonistic autoantibodies released by the placenta could cause severe vasoconstriction. Previously it has 
been proposed that besides activating the AT-1 receptor, increased levels of placental AT-1-agonistic autoantibodies may stimulate endothelial cells to produce ET-1, which may further trigger the genesis of preeclamptic hypertension (30).

The increased contractility of arteries after 1 hour pre-incubation with PSMs corresponds to a similar increased vascular contractility to TXA2, AngII and ET-1 of omental arteries observed in women suffering from PE (31-33). Therefore, it will be one of the goals of our model to test the vascular effect of PSMs on omental arteries, which will further unravel the complex pathophysiological process of PE.

Although the exact mechanism of PE is still enigmatic, there is a growing body of evidence that vasoactive PSMs obtained under hypoxia promote a TXA2 and prostaglandins disbalance and that this is pivotal in the development of PE-induced hypertension (33). It can explain the increased maternal vascular contractility to vasoactive compounds after a PE-complicated pregnancy (34). Our data indicate that hypoxia is one of the triggers that lead to the release of vasoactive PSMs into the maternal circulation and forming the culprit of the acute increase in blood pressure during pregnancy as well as the increased risk for high blood pressure in later life of the mother.

The low inter- and intra-coefficient of the variance of the PSM extraction procedure of 4.5 and $0.5 \%$ respectively indicates the applicability of the PSM generation procedure under severe hypoxia and its induced vaso-reactivity in chorionic arteries.

Knowing key players of PE like oxidative and inflammatory stress are known as powerful stimuli for altered protein expression (35). A better understanding of the changes in protein expression will allow the development of novel biomarkers for the prediction and diagnosis and potential treatment targeting key aspects of the pathophysiology (35). The experiment with the dietary bioactive compound quercetin underlines the potential of our in vitro model. When quercetin, the most abundant flavonoid in our diet, was added to the HEPES buffer containing the placental tissue before exposure to severe hypoxia, the direct vasocontraction and increased vascular contractility to U46619 induced by the released PSMs was concentration-dependently reduced. Although the exact mode of action of quercetin needs to be elucidated, this indicates that bioactive compounds in our diet might be useful in targeting the different elements of PE.

Further experiments can include exposure of placental tissue to inflammatory stress factors like TNF- $\alpha$ (Tumor necrosis factor-alpha) to determine whether only hy- 
poxia triggers the release of vasoactive compounds by syncytiotrophoblasts cells. Previous studies showed evidence that HIF-1 $\alpha$ (Hypoxia-inducible factor 1-alpha), considered as the master transcriptional regulator of the cellular and developmental response to hypoxia itself can be stimulated and stabilized by inflammatory stimuli like lipopolysaccharide (LPS) under normoxic conditions. This shows a potential overlap between inflammatory and hypoxic responses in preeclampsia $(16,36,37)$. Besides changes in vascular reaction, specific inflammatory cytokines and vesicles characteristics could be determined in the conditioned medium. Redman et al. speculated, that in PE due to oxidative and inflammatory stress, syncytiotrophoblast cells are activated to release larger microvesicles that exhibit proteins, which have pro-inflammatory, anti-angiogenic and procoagulant activity that could contribute to exaggerated systemic inflammation in PE (38). It is now recognized that the exact nature and composition of syncytiotropoblast microvesicles has yet to be defined. However, ex vivo studies have shown that these microvesicles play a key role in normal maternal immunomodulation that results in the regulation of the T-helper cells and may be thus responsible for the Th1 skewness in PE $(39,40)$.

\section{Conclusion}

An in vitro model for PE was set up using healthy term placentae and chorionic arteries to investigate the direct and sustained vascular consequences of PSMs released under hypoxic stress conditions in the placenta as observed in PE. The model is also suitable to test the potential beneficial effects of food compounds like quercetin to prevent the vascular consequences of $\mathrm{PE}$ and to elucidate their mechanism of action. Our in vitro model opens avenues to develop interventions aimed at treating or even preventing preterm birth in PE.

\section{Acknowledgements}

This study was supported by NUTRIM Graduate Program. There was no additional external funding received for this study. 


\section{References}

1. Saleh L, Verdonk K, Visser W, van den Meiracker AH, Danser AH. The emerging role of endothelin-1 in the pathogenesis of pre-eclampsia. Ther Adv Cardiovasc Dis. 2016.

2. Drenthen W, Boersma E, Balci A, Moons P, Roos-Hesselink JW, Mulder BJ, et al. Predictors of pregnancy complications in women with congenital heart disease. Eur Heart J. 2010;31(17):2124-32.

3. Steegers EA, von Dadelszen P, Duvekot JJ, Pijnenborg R. Pre-eclampsia. Lancet. 2010;376(9741):631-44.

4. Parets SE, Bedient CE, Menon R, Smith AK. Preterm birth and its long-term effects: methylation to mechanisms. Biology (Basel). 2014;3(3):498-513.

5. Morton JS, Cooke CL, Davidge ST. In Utero Origins of Hypertension: Mechanisms and Targets for Therapy. Physiol Rev. 2016;96(2):549-603.

6. Nakimuli A, Chazara O, Farrell L, Hiby SE, Tukwasibwe S, Knee O, et al. Killer cell immunoglobulin-like receptor (KIR) genes and their HLA-C ligands in a Ugandan population. Immunogenetics. 2013;65(11):765-75.

7. Moffett A, Hiby SE. How Does the maternal immune system contribute to the development of pre-eclampsia? Placenta. 2007;28 Suppl A:S51-6.

8. Burton GJ, Woods AW, Jauniaux E, Kingdom JC. Rheological and physiological consequences of conversion of the maternal spiral arteries for uteroplacental blood flow during human pregnancy. Placenta. 2009;30(6):473-82.

9. Kaufmann P, Black S, Huppertz B. Endovascular trophoblast invasion: implications for the pathogenesis of intrauterine growth retardation and preeclampsia. Biol Reprod. 2003;69(1):1-7.

10. Roberts JM, Escudero C. The placenta in preeclampsia. Pregnancy Hypertens. 2012;2(2):72-83.

11. Crocker I. Gabor Than Award Lecture 2006: pre-eclampsia and villous trophoblast turnover: perspectives and possibilities. Placenta. 2007;28:S4-S13.

12. Heazell AE, Taylor NN, Greenwood SL, Baker PN, Crocker IP. Does altered oxygenation or reactive oxygen species alter cell turnover of BeWo choriocarcinoma cells? Reprod Biomed Online. 2009;18(1):111-9.

13. Wu F, Tian FJ, Lin Y. Oxidative Stress in Placenta: Health and Diseases. Biomed Res Int. 2015;2015:293271.

14. Myatt L, Cui X. Oxidative stress in the placenta. Histochem Cell Biol. 2004;122(4):369-82.

15. Huppertz B. Placental Villous Trophoblast: the Altered Balance Between Proliferation and Apoptosis Triggers Pre-eclampsia Journal of Reproductive Medicine and Endocrinology 2006;J. Reproduktionsmed. Endokrinol 2006; 3 (2), 103- 
108.

16. Redman CW, Sargent IL. Placental stress and pre-eclampsia: a revised view. Placenta. 2009;30 Suppl A:S38-42.

17. Goulopoulou S, Davidge ST. Molecular mechanisms of maternal vascular dysfunction in preeclampsia. Trends Mol Med. 2015;21(2):88-97.

18. Redman CW, Sargent IL. Preeclampsia and the systemic inflammatory response. Semin Nephrol. 2004;24(6):565-70.

19. Luft FC. Proinflammatory effects of angiotensin II and endothelin: targets for progression of cardiovascular and renal diseases. Curr Opin Nephrol Hypertens. 2002;11(1):59-66.

20. Newson AJ. Ethical aspects arising from non-invasive fetal diagnosis. Semin Fetal Neonatal Med. 2008;13(2):103-8.

21. Blehar MC, Spong C, Grady C, Goldkind SF, Sahin L, Clayton JA. Enrolling pregnant women: issues in clinical research. Womens Health Issues. 2013;23(1):e3945.

22. Boots AW, Haenen GR, Bast A. Health effects of quercetin: from antioxidant to nutraceutical. Eur J Pharmacol. 2008;585(2-3):325-37.

23. Hytten F. Blood volume changes in normal pregnancy. Clin Haematol. 1985;14(3):601-12.

24. Perry IJ, Beevers DG, Whincup PH, Bareford D. Predictors of ratio of placental weight to fetal weight in multiethnic community. BMJ. 1995;310(6977):4369.

25. Gillham JC, Kenny LC, Baker PN. An overview of endothelium-derived hyperpolarising factor (EDHF) in normal and compromised pregnancies. Eur J Obstet Gynecol Reprod Biol. 2003;109(1):2-7.

26. Lemkens P, Spijkers LJ, Meens MJ, Nelissen J, Janssen B, Peters SL, et al. Dual NEP/ECE inhibition improves endothelial function in mesenteric resistance arteries of 32-week-old SHR. Hypertens Res. 2017;40(8):738-45.

27. Nishikawa Y, Stepp DW, Chilian WM. Nitric oxide exerts feedback inhibition on EDHF-induced coronary arteriolar dilation in vivo. Am J Physiol Heart Circ Physiol. 2000;279(2):H459-65.

28. Wilkes BM, Hollander AM, Sung SY, Mento PF. Cyclooxygenase inhibitors blunt thromboxane action in human placental arteries by blocking thromboxane receptors. Am J Physiol. 1992;263(4 Pt 1):E718-23.

29. Zhao YWaS. Vascular Biology of the Placenta, Chapter 8: Vasoactivators and Placental Vasoactivity2010.

30. George EM, Granger JP. Endothelin: key mediator of hypertension in preeclampsia. Am J Hypertens. 2011;24(9):964-9.

31. Belfort MA, Saade GR, Suresh M, Kramer W, Vedernikov YP. Effects of se- 
lected vasoconstrictor agonists on isolated omental artery from premenopausal nonpregnant women and from normal and preeclamptic pregnant women. Am J Obstet Gynecol. 1996;174(2):687-93.

32. Everett RB, Worley RJ, MacDonald PC, Gant NF. Effect of prostaglandin synthetase inhibitors on pressor response to angiotensin II in human pregnancy. J Clin Endocrinol Metab. 1978;46(6):1007-10.

33. Walsh S, Glob. libr. women's med., (ISSN: 1756-2228) 2011; DOI 10.3843/ GLOWM.10315.

34. Walsh SW. Preeclampsia: an imbalance in placental prostacyclin and thromboxane production. Am J Obstet Gynecol. 1985;152(3):335-40.

35. Tannetta D, Sargent I. Placental disease and the maternal syndrome of preeclampsia: missing links? Curr Hypertens Rep. 2013;15(6):590-9.

36. Blouin CC, Page EL, Soucy GM, Richard DE. Hypoxic gene activation by lipopolysaccharide in macrophages: implication of hypoxia-inducible factor 1alpha. Blood. 2004;103(3):1124-30.

37. Possomato-Vieira JS, Khalil RA. Mechanisms of Endothelial Dysfunction in Hypertensive Pregnancy and Preeclampsia. Adv Pharmacol. 2016;77:361-431.

38. Redman CW, Tannetta DS, Dragovic RA, Gardiner C, Southcombe JH, Collett GP, et al. Review: Does size matter? Placental debris and the pathophysiology of pre-eclampsia. Placenta. 2012;33 Suppl:S48-54.

39. Tannetta D, Masliukaite I, Vatish M, Redman C, Sargent I. Update of syncytiotrophoblast derived extracellular vesicles in normal pregnancy and preeclampsia. J Reprod Immunol. 2017;119:98-106.

40. Pillay P, Moodley K, Moodley J, Mackraj I. Placenta-derived exosomes: potential biomarkers of preeclampsia. Int J Nanomedicine. 2017;12:8009-23. 


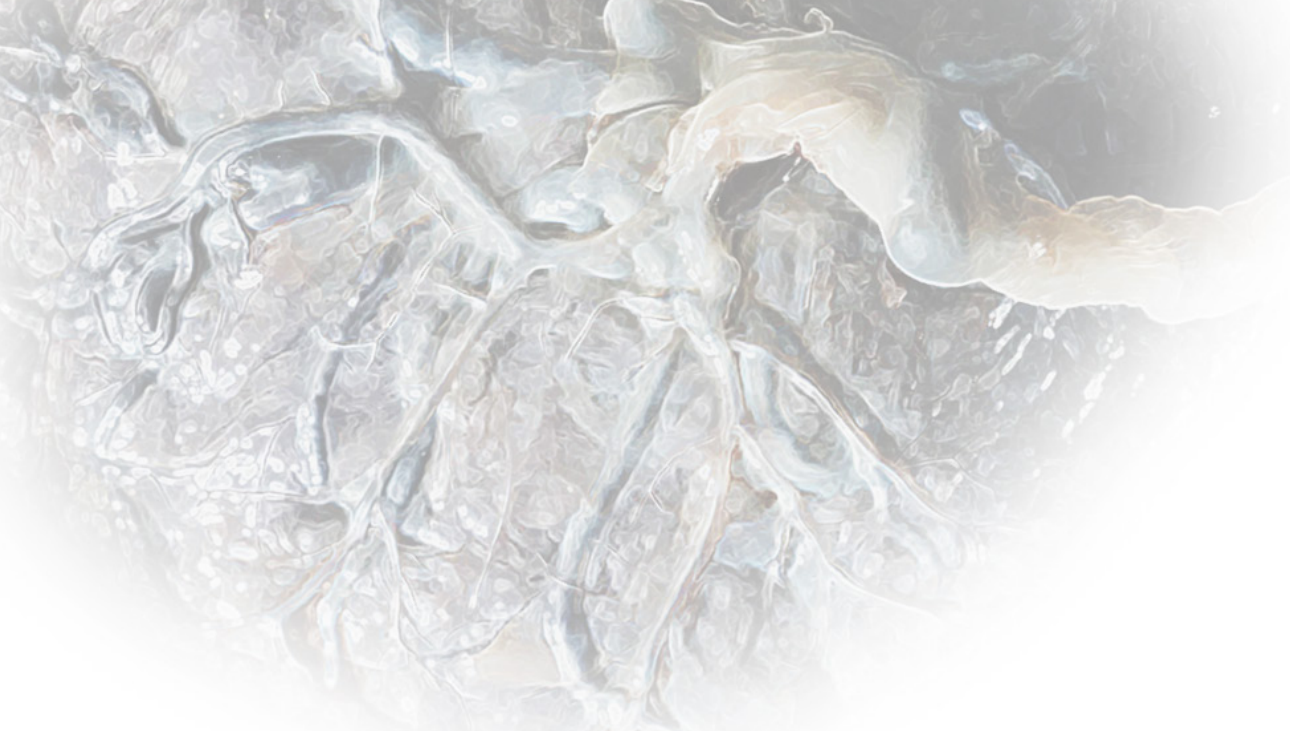




\section{Chapter 6}

\section{P lacental hypoxia-induced alterations in vascular function, morphology and endothelial barrier integrity}

Philippe Vangrieken, Alex H.V. Remels, Salwan Al-Nasiry, Aalt Bast, Ger. M. J. Janssen, Ulrike von Rango, Annemarie Carree, Daan Vroomans, Yannick C.W. Pinckers, Frederik J. van Schooten, Paul M.H. Schiffers

Hypertesion Research. 2020, accepted. 


\section{Abstract}

Preeclampsia is a pregnancy-related disorder characterized by hypertension and proteinuria afflicting $3-10 \%$ of all pregnancies. Although its pathophysiology remains obscure, placental hypoxia-induced oxidative stress and alterations in vascular function, morphology, and endothelial barrier integrity are considered to play a key role in the development of preeclampsia. Placental villous explants of non-complicated placentae and BeWo cells were subjected to hypoxia. The effect of placental hypoxic-conditioned medium (HCM) on intraluminal-induced contraction and endothelial barrier integrity in chorionic arteries was investigated using pressure myography. The impact of BeWo cell HCM on endothelial cell viability, reactive oxygen species formation and inflammation was determined. Alterations in arterial morphology and contractile responsiveness to the thromboxane A2 analogue (U46619) after exposure to placental HCM were examined immunohistochemically and by wire myography, respectively. Intraluminal administration of placental HCM induced vasoconstriction and increased endothelial permeability for $\mathrm{KCl}$, which could be concentration-dependently prevented by quercetin. Placental and BeWo cell HCM decreased endothelial cell viability, increased the production of reactive oxygen species and enhanced the secretion of IL-6 and IL-8. Cross-sectional area of the arterial media was increased upon exposure to placental HCM, which was associated with increased vascular proliferation and contractile responsiveness to U46619, which all could be prevented by the anti-oxidants quercetin and RRR- $\alpha$-tocopherol. This study is the first to comprehensively demonstrate the link between factors secreted by placental cells in response to hypoxia and vascular abnormalities and paves the way for new diagnostic approaches and therapies to better protect the maternal vasculature during and after a preeclamptic-complicated pregnancy. 


\section{Graphical abstract}

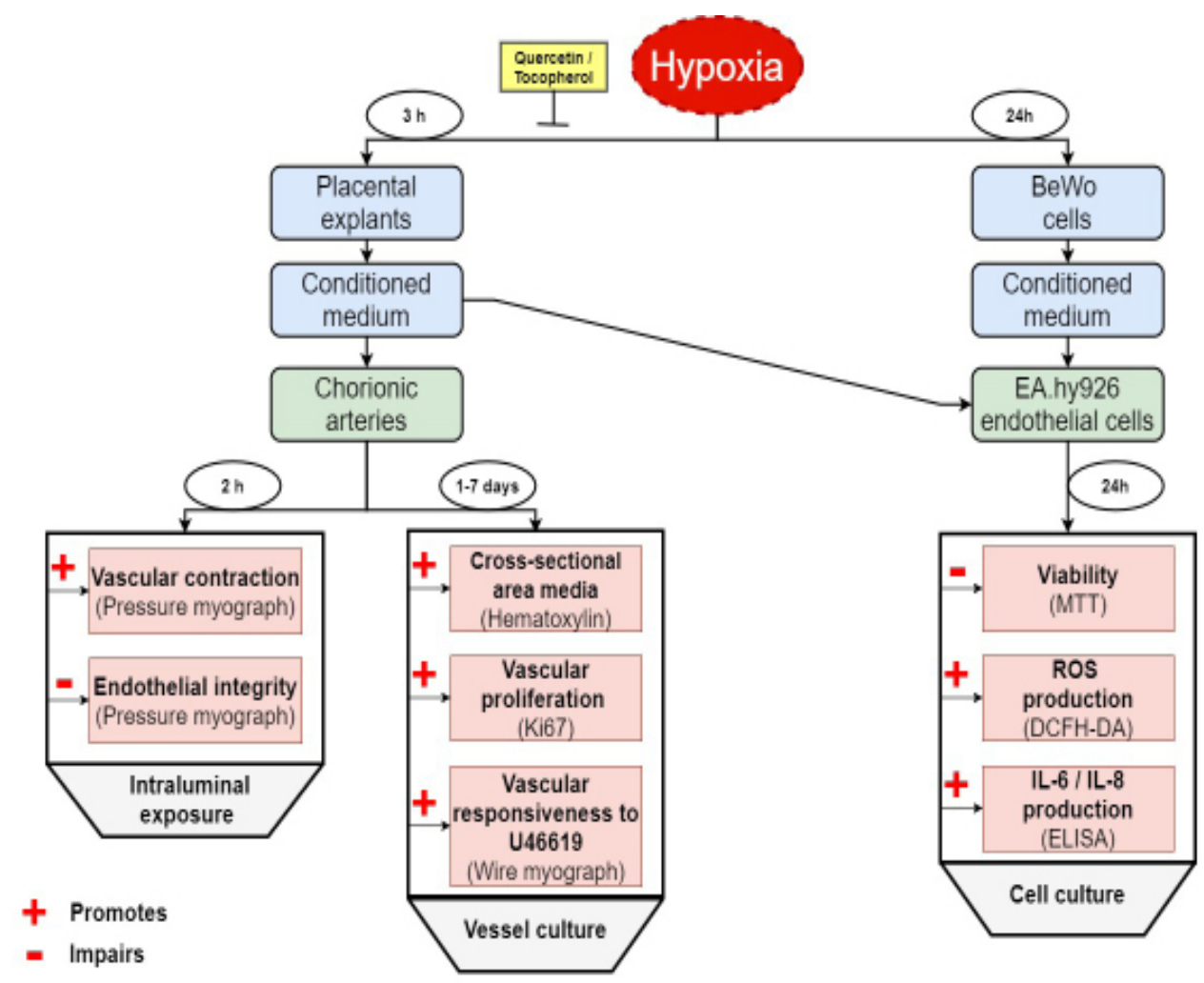




\section{Introduction}

Preeclampsia (PE) is a pregnancy-related disorder afflicting an estimated 3-10\% of all pregnancies worldwide. PE is often associated with fetal intrauterine growth restriction and remains a leading cause of maternal death and perinatal morbidity (1). As its etiology and pathophysiology are not fully understood, aside from the delivery of the fetus, currently there is no available treatment.

One of the pathophysiological hallmarks of PE is severe maternal hypertension, which is occasionally associated with proteinuria and edema in the late second or third trimester (1). Importantly, there are two sub-types of PE: early- and late-onset PE. Early-onset PE, also known as severe PE, is widely acknowledged to have a defect in placentation, while late-onset PE may center around interactions between senescence of the placenta and a maternal genetic predisposition to cardiovascular and metabolic diseases (2). The pathophysiological processes that have been proposed to underlie early-onset PE include defective placentation and apoptosis of invasive cytotrophoblasts causing inadequate remodeling of spiral arteries leading to a decrease in blood flow to the placenta (3-5). Subsequent placental ischemia/hypoxia leading to oxidative stress (4) is believed to result in the production of a variety of placental secreted factors that collectively have profound effects on blood flow and arterial pressure regulation. These include pro- and anti-angiogenic factors such as soluble fms-like tyrosine kinase-1 (sFlt-1; an endogenous inhibitor of vascular endothelial growth factor (VEGF)) and pro-inflammatory cytokines including tumor necrosis factor alpha (TNF- $\alpha$ ) and interleukin 6 (IL-6), and vascular contractile compounds like angiotensin II type 1 receptor agonistic autoantibody (AT $-\mathrm{AA})(1,6-10)$. In animal studies, reduced uterine perfusion (as demonstrated in PE) has been shown to induce hypertension (11-13). In line with these studies, we previously demonstrated that factors released by a hypoxic placenta increase vasoconstriction via the Angiotensin II receptor type 1 (AT1) and endothelin 1 (ET-1) receptor and increased vascular contractility to a thromboxane-A2 (TXA2) agonist analogue (U46619) in chorionic arteries mounted in a wire myograph. When the exposure of placental explants to hypoxia was combined with the flavonoid quercetin, the acute and sustained vascular effects could be prevented in a concentration-dependent manner (6). Whether or not these effects on vascular contractility were endothelial-dependent remains to be elucidated, since the myograph setting as used in our previous work is not intraluminal-specific (6).

Quercetin was selected for this study, since it is the most abundant flavonoid found in our diet. To confirm if the protective effect of quercetin is due to its antioxidant prop- 
erties, RRR- $\alpha$-tocopherol, was tested in our study, which is the naturally occurring and most active form and which have already been used as a fertility enhancement and to improve uterine artery blood flow (14-16). The parameters used in our study may serve as a tool to explore the benefits of dietary compounds on specific targets central to the pathogenesis of PE. There is growing attention in dietary-directed prevention of diseases and consumption with diet appears to be save $(17,18)$. RRR- $\alpha$-tocopher$\mathrm{ol}$ is a fat-soluble nutrient that is extremely important during from time of conception to the postnatal development of the infant. The mechanisms involved in its placental uptake appear to be allowed by the presence of lipoprotein receptors (LDL-receptor, VLDL-receptor, scavenger receptor class B type I) together with lipoprotein lipase at the placental barriers (19). The ingestion of colostrum which contains very high levels of RRR- $\alpha$-tocopherol is therefore of utmost importance to supply the newborn with an essential defense against oxygen toxicity (19). The use of RRR- $\alpha$-tocopherol as a therapeutic agent in PE, has been discussed in numerous papers (19). Our data will help in the selection of effective bioactives and subsequently establish dietary recommendations to treat/prevent vascular consequences of PE.

Besides the direct effects on contractility of the vessels during pregnancy, resulting in maternal hypertension, a preeclamptic pregnancy may also irreversibly modify the maternal vascular system, leaving the mother at increased risk for cardiovascular diseases in later life. Indeed, strikingly, women who have experienced PE are twice as likely to succumb to cardiovascular-related events in later life compared to those that have had normal pregnancies (20), the reason for this remains unclear (21). Using laser Doppler and iontophoresis, impaired function of the endothelium and smooth muscle of the skin microvasculature in women which experienced PE was demonstrated 15-25 years postpartum (22). In the major blood vessel of the upper arm (brachial artery), endothelial function was found to be impaired in women in their early postpartum period (median, 3 years), which could be ameliorated using a systemically acting antioxidant ascorbic acid (23).

Understanding the link between a hypoxic placenta and its resulting vascular abnormalities could help in the identification of new serum biomarkers for the prediction of $\mathrm{PE}$ and in the design of new diagnostic approaches for a better clinical management. It will also improve our knowledge on the sustained maternal vascular consequences opening new postpartum treatment and follow-up strategies. Because of the difficulty to perform mechanistic studies on the complex interplay between the placenta and the maternal vascular system in pregnant women, an in vitro setting using human placentae and chorionic arteries as used in our study served as a physiological relevant tool. As recently recognized by Burton et al., the synergistic effect 
of placental secreted factors may explain why PE has proved so hard to treat, as it is likely that the peripheral aspects of the syndrome are caused by a complex mix of factors rather than any one mediator alone (2).

The aim of the present study, therefore, was to examine the effect of placental hypoxic-conditioned medium (HCM) on vascular function, morphology, and endothelial barrier integrity. To investigate if the alterations on endothelial barrier integrity found were trophoblast specific, the effect of both placental and BeWo cells HCM on endothelial viability, inflammation, and oxidative stress was tested. 


\section{Materials and methods}

\section{Clinical subject characteristics}

For the generation of placental-conditioned medium (CM), 3 term placentae were collected from caesarean deliveries by the Department of Obstetrics and Gynaecology at Maastricht University Medical Center+. Exclusion criteria were hypertension (pre-existing or onset during pregnancy) with or without proteinuria as defined by the International Society for the study of Hypertension in Pregnancy (ISSHP), fetal chromosomal anomalies, multiple gestation, obesity and (pre)gestational diabetes. In addition, 66 placentae were used for the isolation of chorionic arteries. Chorionic arteries were chosen, since they contain AT-1, ET-1, serotonin (5-HT) and TXA2 receptors and thus are relevant in respect of identifying vasoactive compounds related to PE 24. The umbilical-placental vessels have the particular property of lacking autonomic innervation, which in fact permits investigation of direct actions of vasoactive compounds and drugs 24. All experiments were approved by the Medical Ethics Committee Academic Hospital Maastricht and Maastricht University (METC 16-4-047).

\section{Villous explant isolation and collection placental of CM}

As described earlier by Vangrieken et al. (6), three placentae from non-complicated caesarean deliveries were processed directly after delivery. Per placenta, five villous explants of $30 \mathrm{~g}$ were collected from the central region of the placenta at the maternal side. Subsequently, the basal plate of the specimens was removed and the remaining tissue was rinsed in a HEPES solution ( $\mathrm{NaCl} 143.3 \mathrm{mM}, \mathrm{KCl} 4.7 \mathrm{mM}, \mathrm{MgSO}_{4} 1.2$ $\mathrm{mM}, \mathrm{KH}_{2} \mathrm{PO}_{4} 1.2 \mathrm{mM}, \mathrm{CaCl}_{2} 2.5 \mathrm{mM}$, glucose $5.5 \mathrm{mM}$, and HEPES $15 \mathrm{mM}$ ). Specimens (15 in total) were then separately transferred into a bottle containing $200 \mathrm{ml}$ pre-warmed $\left(37^{\circ} \mathrm{C}\right)$ HEPES buffer, kept in a warm water bath $\left(37^{\circ} \mathrm{C}\right)$ and aerated under a standard culture condition $\left(21 \% \mathrm{O}_{2}\right.$, referred as the control condition) or under a hypoxic condition $\left(1 \% \mathrm{O}_{2}\right)$ in the presence/absence of 3 or $10 \mu \mathrm{M}$ quercetin (which is comparable with plasma levels of quercetin found in mice upon nutritional supplementation (25)) or $20 \mu \mathrm{M}$ RRR- $\alpha$-tocopherol (the relevant physiological plasm concentration upon nutritional supplementation of the naturally occurring and most active $\alpha$-tocopherol stereoisomer (26)) for $3 \mathrm{~h}$. Solutions were then centrifuged at $500 \mathrm{~g}$ for $10 \mathrm{~min}$ at $4{ }^{\circ} \mathrm{C}$. Resulting placental CM was stored in aliquots of $3 \mathrm{ml}$ at $-80{ }^{\circ} \mathrm{C}$ until use.

\section{Mounting of chorionic arteries into a pressure myograph}

For pressure myography, freshly isolated chorionic arteries of 100-400 $\mu \mathrm{m}$ width and $\geq 1 \mathrm{~cm}$ length were used. A stainless steel wire (diameter of $40 \mu \mathrm{m}$ ) was lead 
through the lumen of the arterial segment. The isolated artery was cleaned by cutting away the connective perivascular tissue and stored in a HEPES buffer $\left(4{ }^{\circ} \mathrm{C}\right)$ and used within $24 \mathrm{~h}$ after isolation. Subsequently, a dual vessel chamber $(\mathrm{CH}-2$, Living Systems Instrumentation, Vermont, USA) was used. The vessel chamber was filled with $7 \mathrm{ml}$ HEPES buffer and kept at $37^{\circ} \mathrm{C}$ by a temperature controller for dual chamber (TC-09D Living Systems Instrumentation). The isolated artery was then mounted onto two glass cannulas of the vessel chamber. The artery was secured using two double knotted strings on both cannulas. Simultaneously, a pressure servo controller with a peristaltic pump (PS-200, Living Systems Instrumentations) was connected to the cannulas. The pressure pump was infused with HEPES buffer at 10 $\mathrm{mmHg}$ and subsequently, increased to an intraluminal pressure of $50 \mathrm{mmHg}$, which was kept constant during the experiments. To infuse solutions of interest, a flow control peristaltic pump (FC, Living Systems Instrumentation) was used at a flow rate of $210 \mu \mathrm{l} / \mathrm{min}$. A video camera (TVC, Living Systems Instrumentation), on the inverted microscope, visualized the artery in the vessel chamber. A video dimension analyzer (VDA-10, Living Systems Instrumentation) connected to the monitor, measured the lumen diameter. The diameter change was expressed as the delta diameter change $(\mu \mathrm{m})$. Data measured by the VDA were transferred (Data acquisition transformer, Living System Instrumentation) and analyzed with the use of Wintech data acquisition (ASUS).

\section{Testing vascular contractile responsiveness and endothelial bar- rier integrity upon intraluminal exposure to placental $C M$}

To determine the maximal contraction, chorionic arteries were first extraluminally pre-contracted by $\mathrm{KCl}(62.5 \mathrm{mM})$. Subsequently, after complete relaxation, chorionic arteries were intraluminally exposed to $\mathrm{KCl}(62.5 \mathrm{mM})$ and flushed with HEPES for baseline conditions. Next, chorionic arteries were infused intraluminally for $2 \mathrm{~h}$ with undiluted placental $\mathrm{CM}$ of the control culture condition $\left(21 \% \mathrm{O}_{2}\right.$, control-conditioned medium: $\mathrm{CCM})$, the hypoxic condition $\left(1 \% \mathrm{O}_{2}\right.$, hypoxic-conditioned medium: $\mathrm{HCM}$ ) or the $\mathrm{CM}$ of placenta exposed to hypoxia in the presence of 3 or 10 $\mu \mathrm{M}$ quercetin ( $\mathrm{n}=8$ for each condition). To determine the vascular contractile effect of placental CM, arterial diameter was recorded after $2 \mathrm{~h}$. Chorionic arteries were subsequently flushed again with HEPES for baseline conditions. Finally, chorionic arteries were again intraluminally exposed to $\mathrm{KCl}(62.5 \mathrm{mM})$. Differences between the intraluminally $\mathrm{KCl}$-induced contraction before and after exposure to the placental CM was used as a parameter for endothelial barrier integrity.

\section{Chorionic artery isolation and culture exposure to placental CM} Areas from the chorionic plate containing chorionic arteries were macroscopically 
selected and excised in placentae from both vaginal and caesarean deliveries. The dissected specimens were immediately immersed in HEPES solution $\left(4^{\circ} \mathrm{C}\right)$ and pinned in a Sylgaard dish with the fetal side orientated upwards. Chorionic arteries (second and third-order branches of the umbilical cord) were dissected under a microscope. From each placenta, one isolated artery was cut into arterial segments of $2 \mathrm{~mm}$. Arterial segments were first pre-warmed $\left(37^{\circ} \mathrm{C}\right)$ in basal Dulbecco's Modified Eagle Medium (DMEM; D-glucose and L-glutamine $1 \mathrm{~g} / \mathrm{L}$ and HEPES and pyruvate 25 $\mathrm{mM}$, GIBCO, Carlsbad, CA, USA) containing antibiotics $(1000 \mathrm{U} / \mathrm{ml}$ penicillin and $1000 \mu \mathrm{g} / \mathrm{ml}$ streptomycin; both from GIBCO) for $2 \times 5 \mathrm{~min}$ at $37^{\circ} \mathrm{C}$. Subsequently, arterial segments were cultured under a humidified atmosphere containing $21 \% \mathrm{O}_{2}$, $5 \% \mathrm{CO}_{2}$ at $37{ }^{\circ} \mathrm{C}$ for 1,3 or 7 days in a 24 -wells plate containing $400 \mu 1$ placental $\mathrm{CM}$ and $600 \mu \mathrm{l}$ basal medium containing antibiotics $(50 \mathrm{U} / \mathrm{ml}$ penicillin and $50 \mu \mathrm{g} /$ $\mathrm{ml}$ streptomycin) $(40 \%(\mathrm{vol} / \mathrm{vol}))$. The diluted placental CM was refreshed daily.

\section{Mounting and examining of cultured arterial segments in a wire myograph}

Cultured arterial segments of $2 \mathrm{~mm}$ were mounted in a myograph for the recording of isometric tension development (DMT, Aarhus, Denmark). The organ chamber was filled with Krebs Ringer bicarbonate buffer (KRB) containing (in $\mathrm{mM}$ ): $\mathrm{NaCl}$ 118.3, $\mathrm{KCl} 4.7, \mathrm{CaCl}_{2} 2.5, \mathrm{MgSO}_{4} 1.2, \mathrm{KH}_{2} \mathrm{PO}_{4} 1.2, \mathrm{NaHCO}_{3} 25.0$ and glucose 11.1 $(\mathrm{pH}=7.4)$ and aerated with $95 \% \mathrm{O}_{2}$ and $5 \% \mathrm{CO}_{2}$. Two stainless steel wires (diameter of $40 \mu \mathrm{m}$ ) were inserted in the lumen of the arterial segments and were connected to a displacement device and an isometric force transducer. All arterial preparations were stretched to a diameter at which their individual mechanical performance was maximal. To achieve this, the arterial diameter was stepwise increased and preparations were intermittently exposed to $65.5 \mathrm{mM}$ potassium-KRB until maximal contractile responses were obtained. Contractile responses were expressed as the increase in wall tension (increase in force/twice the segment length; $\mathrm{N} / \mathrm{m}$ ) relative to the response to the maximal concentration of the thromboxane-A2 (TXA2) agonist analogue (U46619, Enzo Life sciences, USA) of the control condition.

\section{Testing vascular contractile responsiveness to U46619 after ex- posure to placental CM}

Cultured arterial segments were contracted by U46619 in logarithmically increasing doses $0.1 \mathrm{nM}-1 \mu \mathrm{M}$. After washing and equilibration ( $\pm 30 \mathrm{~min})$, chorionic arteries were pre-contracted by U46619 (30 nM). Subsequently, vasodilation was induced by logarithmically increasing doses of sodium nitroprusside $(0.01 \mathrm{nM}-30 \mu \mathrm{M}$, SNP, Sigma Aldrich, Zwijndrecht, the Netherlands). After the experiments, arterial segments were formalin-fixed for paraffin-embedding. 


\section{Immunohistochemistry on chorionic arteries exposed to placen- tal $C M$}

To identify the proliferative activity of the chorionic arteries exposed to the placental CM, following the myograph experiments, cultured arterial segments were immunohistochemically analyzed using commercially-available antibodies following standard manual protocols on formalin-fixed, paraffin-embedded (FFPE) tissue. The slides were incubated with antibodies for mindbomb E3 ubiquitin protein ligase 1 (MIB-1, Ki-67, Dako, catalogue no. M7240) (Agilent Technologies, Glostrup, Denmark), according to routine protocols. Briefly, $5 \mu \mathrm{m}$ sections of paraffin-embedded tissue were first deparaffinized with absolute Xylene and endogenous peroxidase was blocked in $0.3 \% \mathrm{H}_{2} \mathrm{O}_{2} /$ methanol for $10 \mathrm{~min}$. Sections were subsequently hydrated via graded ethanol series (100\%-50\% PBS (Phosphate Buffered Saline)). Arterial sections were boiled in a sodium citrate solution (10 mM, pH 6.0) for $30 \mathrm{~min}$, cooled at room temperature and washed 3 times in PBS. All subsequent steps were performed at room temperature. Sections were then incubated with PBST (Phosphate buffer with Tween 20) $/ 0.5 \%$ BSA (bovine serum albumin) for $30 \mathrm{~min}$ to minimize nonspecific antibody binding. Sections were incubated with Ki-67 1:40 in PBST 15\% BSA) for $4 \mathrm{~h}$. After washing 3 times with PBST, a secondary antibody: goat anti-mouse (GAM-biotin Vector BA9200; 1:500 in PBST) was allowed to bind for $30 \mathrm{~min}$ at room temperature, followed by 3 washes in PBST. HRP coupled Avidin/Streptavidin-biotin complex (ABC (Avidin-biotin-complex) kit Elite, Vectastain PK6100, Vector Laboratories Inc., 30 Ingold Road, Burlingama, CA 94010) was used to amplify the signal and incubated for $30 \mathrm{~min}$ at room temperature, followed by 3 washes in PBST. Sections were then incubated with the substrate 3,3'-diaminobenzindine (DAB). Staining was stopped with water. In parallel, for the same placental chorionic arteries, a second $5 \mu \mathrm{m}$ arterial section of the same artery was subjected to a hematoxylin staining for the measurement of the cross-sectional area of the lumen, media, and adventitia. Finally, sections were dehydrated via a graded alcohol series (70\%, 90\%, 96\%, 2x 100\%) and covered with Entellan (Merck KGaA, 64271 Darmstadt, Germany). For negative controls, the first antibody was replaced by a buffer.

\section{Histological analysis of cultured chorionic arteries exposed to placental $C M$}

All arterial cross-sections were examined at a magnification of $10 \mathrm{x}$ using a light microscope (Leica DM4 B, Leica Microsystems GmbH, Ernst-Leitz-Strasse 17-37, 35578 Wetzlar, Germany). Ki-67-positive nuclei were counted in Ki-67 stained arterial cross-sections and area of the lumen, media and adventitia were measured in hematoxylin stained arterial cross-sections using Leica-QWin standard software. 


\section{Cell culture of BeWo cells and collection of CM}

BeWo cells, a trophoblast cell line (ECACC, Porton Down, Salisbury, UK, No 86082803) were cultured according to the manufacture's protocol. Briefly, BeWo cells were cultured in Ham's F12 (Kaighn's) growth medium (glutamine $2 \mathrm{mM}$ and D-glucose $7 \mathrm{mM}$, GIBCO, Carlsbad, CA, USA) enriched with $10 \%$ (vol $/ \mathrm{vol}$ ) fetal bovine serum (FBS, SIGMA, St. Louis, MO, USA) and antibiotics $(50 \mathrm{U} / \mathrm{ml}$ penicillin and $50 \mu \mathrm{g} / \mathrm{ml}$ streptomycin; both from GIBCO). Cells were routinely maintained in $175-\mathrm{cm}^{2}$ falcon flasks at $\mathrm{pH} 7.4$ under $5 \% \mathrm{CO}_{2}$ and $95 \%$ humidity at $37{ }^{\circ} \mathrm{C}$ and passaged when reaching confluency of $70-80 \%$. For medium transfer experiments, $24 \mathrm{~h}$ after cell seeding in 6-wells plates (3x105 cells/well in $2 \mathrm{ml}$ growth medium), cells were cultured for $24 \mathrm{~h}$ under a standard culture (control) condition $\left(21 \% \mathrm{O}_{2}\right.$, $\left.5 \% \mathrm{CO}_{2}\right)$ or a hypoxic condition $\left(1 \% \mathrm{O}_{2}, 5 \% \mathrm{CO}_{2}\right)$, whereafter $\mathrm{CM}$ was collected and stored at $-80^{\circ} \mathrm{C}$ until use.

\section{Exposure of EA.hy926 cells to placental or BeWo cell CM}

EA.hy926 cells, a vascular endothelial cell line derived by fusing primary human umbilical vein endothelial cells (HUVEC) with a thioguanine-resistant clone of A549 (ATCC CRL-2922, Manassa, Virginia, USA) were cultured according to the manufacture's protocol. Briefly, cells were cultured in DMEM (Dulbecco's Modified Eagle Medium, $4 \mathrm{mM}$ L-glutamine, $4500 \mathrm{mg} / \mathrm{L}$ glucose, $1 \mathrm{mM}$ sodium pyruvate, and $1500 \mathrm{mg} / \mathrm{L}$ bicarbonate enriched with $10 \%$ (vol/vol) FBS, antibiotics $(50 \mathrm{U} / \mathrm{ml}$ penicillin and $50 \mu \mathrm{g} / \mathrm{ml}$ streptomycin; both from GIBCO) and 1X HAT supplements (GIBCO). Cells were routinely maintained in $175 \mathrm{~cm}^{2}$ falcon flasks at $\mathrm{pH} 7.4$ under $5 \% \mathrm{CO}_{2}$ and $95 \%$ humidity at $37{ }^{\circ} \mathrm{C}$ and passaged when reaching confluency of 70 $80 \%$. Medium was refreshed every two days. For the CM exposure of EA.hy 926 cells, $\mathrm{CM}$ of the control (CCM) and hypoxic condition (HCM) was first diluted $40 \%$ (vol/vol) with DMEM basal medium. Subsequently, $24 \mathrm{~h}$ after EA.hy926 cell seeding in 6-wells plates (1x105 cells/well in $2 \mathrm{ml}$ growth medium), medium was replaced by the diluted placental or BeWo CCM or HCM and incubated for $24 \mathrm{~h}$.

\section{EA.hy926 cell viability upon exposure to placental or BeWo cell CM}

Cell viability was assessed using the MTT (3-(4,5-dimethylthiazol-2-yl)-2,5-diphenyltetrazolium bromide) assay. After $24 \mathrm{~h}$ exposure of EA.hy926 cells to DMEM basal medium, which was used as positive control or placental or BeWo CCM or HCM, supernatant was removed and $150 \mu$ of MTT solution $(0.5 \mathrm{mg} / \mathrm{ml}$ in DPBS) was added to the cells after which the plate was incubated in the dark at $37{ }^{\circ} \mathrm{C}$ for $1 \mathrm{~h}$. After $1 \mathrm{~h}$, the MTT solution was removed from the wells and $150 \mu \mathrm{l}$ of DMSO (dimethylsulfoxide) was added to each well. After 10 min incubation at room tem- 
perature, absorbance was measured at $\lambda=540 \mathrm{~nm}$.

\section{Quantification of IL 6 and IL 8 secretion by EA.hy926 cells ex- posed to placental or BeWo cell CM}

After 24 h exposure of EA.hy926 cells to placental or BeWo cell CCM or HCM, supernatant was removed and replaced by DMEM basal medium, which was collected after $24 \mathrm{~h}$ for cytokine detection. Human IL-8 (DY208) and human IL-6 (DY206) DuoSet ELISA kits (R\&D Systems, Abingdon, UK) were used and the analyses were carried out in accordance with the manufacturer's instructions.

\section{Intracellular ROS levels in EA.hy926 cells exposed to placental or BeWo cell CM}

Intracellular reactive oxygen species (ROS) levels were quantified using the 2',7'-dichlorodihydrofluorescein diacetate (DCFH-DA)-assay. $72 \mathrm{~h}$ after cell seeding in black and clear bottom 96-wells plates (Costar/sigma Aldrich) (1x105 cells/well in $200 \mu \mathrm{l}$ growth medium), cells were washed twice with $150 \mu \mathrm{l} \mathrm{HBSS}$, incubated for $1 \mathrm{~h}$ with $100 \mu \mathrm{H}$ 2DCFH-DA $(50 \mu \mathrm{M})$ and washed twice again with $150 \mu \mathrm{l}$ HBSS. Subsequently, cells were incubated with $200 \mu \mathrm{l}$ of the diluted $(40 \%$ (vol/vol)) placental or BeWo cell CCM or HCM for $24 \mathrm{~h}$ or with $100 \mu \mathrm{M} \mathrm{H}_{2} \mathrm{O}_{2}$, which was used as a positive control. Fluorescence intensity levels of DCF was measured at $\lambda$ excitation $=485 \mathrm{~nm}$ and $\lambda$ emission $=525 \mathrm{~nm}$ and corrected for total protein content and expressed as percentage relative to the maximal (100\%) intracellular ROS formation of the positive control group.

\section{Statistical analysis}

Results are expressed as mean \pm SEM and $n$ refers to the number of placentae from which blood vessels were isolated or the number of independent cell culture experiments. By using GraphPad Prism 8 Software inc. La Jolla, CA, USA, the maximal contractile response (Emax) and the negative logarithm of the half-maximal effective concentration (EC50) of various conditions were calculated. For each comparison, the D'agrostino and Pearson omnibus normality test was used to test normality. Subsequently, to compare the outcome of the control and hypoxic condition, either an unpaired t-test or Mann Whitney test was used accordingly. To compare of the intervention conditions (quercetin or tocopherol) with the hypoxic condition, the ANOVA one-way analysis of the variance was used followed by the multiple comparison Tukey post-hoc test. If not normally distributed, the Kruskal-Wallis test was used followed by the Dunn's multiple comparison post-hoc. A p-value $<0.05$ was considered as significant difference and presented as follows: Ns: $\mathrm{P}>0.05, * \mathrm{P} \leq$ $0.05, * * \mathrm{P} \leq 0.01, * * * \mathrm{P} \leq 0.001$. 


\section{Results}

\section{Intraluminal administration of placental HCM induces vaso- constriction}

As depicted in Figure 1, intraluminal administration of placental HCM, significantly increased vascular contraction ( $+9753 \pm 1183 \%, \mathrm{p}<0.001, \mathrm{n}=8)$ after $2 \mathrm{~h}$, compared to the placental CCM $(n=8)$. Furthermore, when quercetin $(3$ or $10 \mu M)$ was present during the $3 \mathrm{~h}$ exposure of placental explants to hypoxia, the CM-induced vascular contraction showed a concentration-dependent reduction $(-55 \pm 13 \%, \mathrm{p}<0.001$ and $-131 \pm 28 \%, \mathrm{p}<0.001, \mathrm{n}=8$, respectively) compared to HCM condition (Figure 1).

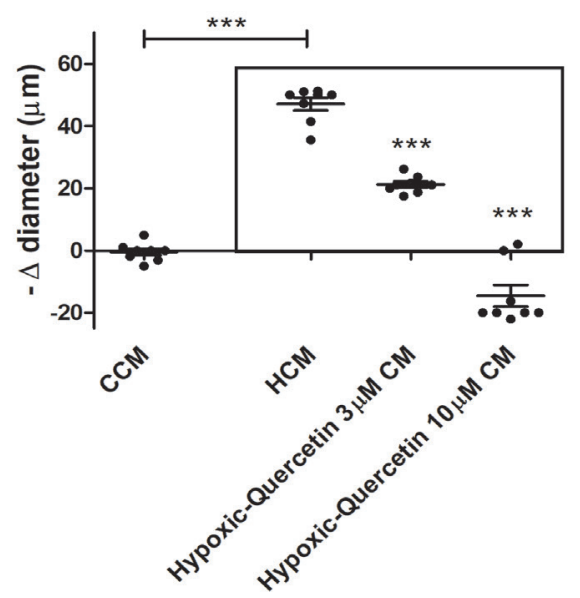

Figure 1. Intraluminal administration of placental HCM induces vasoconstriction. The vascular contractile response $(-\Delta$ diameter $(\mu \mathrm{m}))$ after $2 \mathrm{~h}$ exposure to placental CCM, HCM, hypoxic-quercetin $(3$ or $10 \mu \mathrm{M}) \mathrm{CM}$ of placental chorionic arteries. Data are presented as mean with SEM. Conditions in the black square are compared to the HCM condition. ${ }^{* * *} \mathrm{p} \leq 0.001$. CCM: Control-conditioned medium, CM: Conditioned medium and HCM: Hypoxic-conditioned medium.

\section{Intraluminal administration of placental HCM increased endo- thelial permeability for $\mathrm{KCl}$}

To assess endothelial barrier integrity, intraluminal $\mathrm{KCl}$-induced vascular contraction was tested before and after a $2 \mathrm{~h}$ intraluminal exposure to placental CCM or HCM. As presented in figure 2, $2 \mathrm{~h}$ intraluminal placental CCM did not change endothelial permeability for $\mathrm{KCl}$, since it did not induce a significant vascular contraction $(+35$ $\pm 75 \%, \mathrm{p}=0.2241, \mathrm{n}=8$ ). After intraluminal exposure to placental HCM, intraluminal $\mathrm{KCl}$ induced a significant vascular contraction $(+1089 \pm 276 \%, \mathrm{p}<0.001, \mathrm{n}=8)$, compared to the CCM condition, indicating increased endothelial permeability for 
$\mathrm{KCl}$. Furthermore, when quercetin $(3$ or $10 \mu \mathrm{M})$ was present during the $3 \mathrm{~h}$ exposure of placental explants to hypoxia, the CM significantly reduced intraluminal KCl-induced vascular contraction in a concentration-dependent manner $(-30 \pm 38 \%$, $\mathrm{p}=$ 0.024 and $-91 \pm 21 \%, \mathrm{p}<0.001, \mathrm{n}=8$, respectively) compared to the HCM condition.

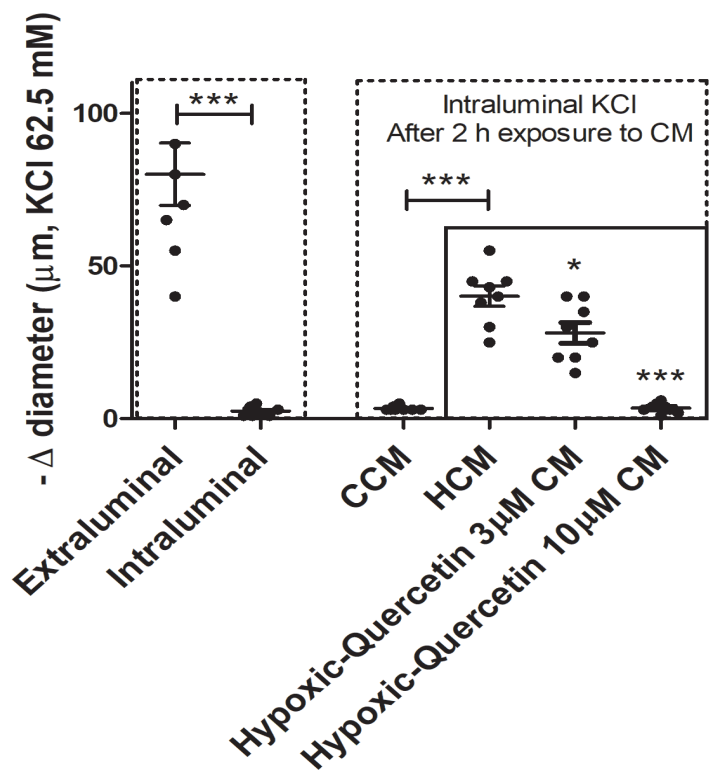

Figure 2. Intraluminal administration of placental HCM increases endothelial permeability for KCl. Extraluminal $\mathrm{KCl}(62.5 \mathrm{mM})$ induced vascular contraction $(-\Delta$ diameter $(\mu \mathrm{m}))$ and intraluminal $\mathrm{KCl}(62.5 \mathrm{mM})$ induced contraction before and after $2 \mathrm{~h}$ intraluminal exposure to CCM, HCM, hypoxic-quercetin $(3$ or $10 \mu \mathrm{M})$ or tocopherol $(20 \mu \mathrm{M}) \mathrm{CM}$ of placental chorionic arteries. Data are expressed as difference in vascular diameter $(\mu \mathrm{m})$ and are presented as mean with SEM. Conditions in the black square are compared to the HCM condition. ${ }^{*} \mathrm{p} \leq 0.05, * * \mathrm{p} \leq 0.01$ and $* * * \mathrm{p} \leq 0.001$. CCM: Control-conditioned medium, CM: Conditioned medium and HCM: Hypoxic-conditioned medium.

\section{Placental HCM decreases endothelial cell viability and increases intracellular ROS formation and inflammation}

To determine the effect of placental CM on endothelial cell viability, ROS formation, and inflammatory status, EA.hy926 endothelial cells were incubated for $24 \mathrm{~h}$ with placental CCM or HCM. After $24 \mathrm{~h}$ exposure to placental HCM, endothelial cell viability was significantly decreased $(-68 \pm 46 \%, \mathrm{p}=0.029, \mathrm{n}=3)$, whereas intracellular ROS formation was significantly increased $(+493 \pm 199 \%, p=0.024, n=3)$ compared to the CCM condition. Furthermore, $24 \mathrm{~h}$ after exposure of EA.hy926 endothelial cells to the placental HCM both IL-6 $(+470 \pm 41 \%, \mathrm{p}<0.001, \mathrm{n}=3)$ and IL-8 $(+566$ $\pm 162 \%, p=0.005, n=3)$ levels in the replaced blank medium were significantly increased compared to the placental CCM condition (Figure 3A-D). 
Placental-conditioned medium

BeWo-conditioned medium
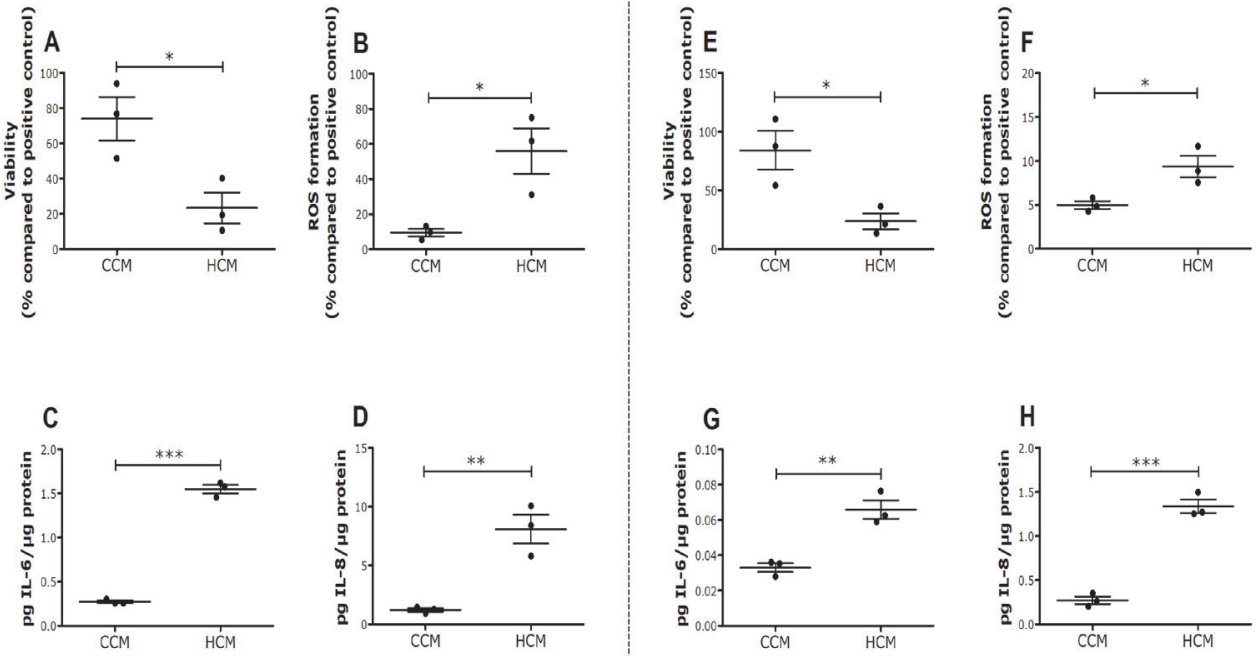

Figure 3. Placental and BeWo cell HCM decreases endothelial cell viability and increases intracellular ROS formation and inflammation. Endothelial cell viability (A), intracellular ROS levels using DCFH-DA (B), cytokine levels of IL-6 (C) and IL-8 (D), assessed in EA.hy926 cells exposed for $24 \mathrm{~h}$ to placental CCM or HCM and endothelial cell viability (E), intracellular ROS levels using DCFHDA (F), cytokine levels of IL-6 (G) and IL-8 (H), assessed in EA.hy926 cells exposed for 24h to BeWo cell CCM or HCM. Data are expressed as percentage compared to the positive control (A, E (DMEM) and $\left.\mathrm{B}, \mathrm{F}\left(100 \mu \mathrm{M} \mathrm{H}_{2} \mathrm{O}_{2}\right)\right)$ or as pg cytokine per $\mu$ g protein $(\mathrm{C}, \mathrm{D}, \mathrm{G}$, and $\mathrm{H})$ and are presented as mean with SEM. ${ }^{*} \mathrm{p} \leq 0.05,{ }^{* *} \mathrm{p} \leq 0.01$ and $* * * \mathrm{p} \leq 0.001$. CCM: Control-conditioned medium, CM: Conditioned medium, HCM: Hypoxic-conditioned medium and ROS: reactive oxygen species.

\section{BeWo cell HCM decreases endothelial cell viability and increas- es intracellular ROS formation and inflammation}

To determine if trophoblast cells play a significant role in the endothelial response to placental CM, EA.hy926 cells were incubated for $24 \mathrm{~h}$ with CCM or HCM from BeWo cells. In line with data obtained with placental HCM, $24 \mathrm{~h}$ exposure to BeWo $\mathrm{HCM}$ significantly decreases endothelial cell viability $(-72 \pm 30 \%, \mathrm{p}=0.027, \mathrm{n}=3)$ and increases intracellular ROS formation $(+89 \pm 27 \%, \mathrm{p}=0.029, \mathrm{n}=3)$ in cultured endothelial cells compared to the BeWo CCM. Furthermore, $24 \mathrm{~h}$ after exposure of EA.hy926 endothelial cells to the BeWo HCM both IL-6 $(+100 \pm 26 \%, p=0.005$, $\mathrm{n}=3)$ and IL-8 $(+393 \pm 28 \%, \mathrm{p}<0.001, \mathrm{n}=3)$ levels in the replaced blank medium were significantly increased compared to the BeWol CCM condition (Figure 3E-H). 


\section{Placental HCM increases vascular cross-sectional area of the media}

To determine if placental HCM affects arterial cross-sectional area, placental chorionic arteries were exposed to placental CCM or HCM up to 7 days after which the cross-sectional area of the lumen, media, and adventitia were assessed. Only the cross-sectional area of the media was significantly increased after 3 and 7 days exposure to placental HCM $(+44 \pm 72 \%, \mathrm{p}<0.040, \mathrm{n}=10$ and $+65 \pm 75 \%, \mathrm{p}<0.010$, $n=10$, respectively) compared to the placental CCM condition ( $n=17$ and $n=18$, respectively) (Figure 4 and supplementary Figure 1). Furthermore, when $10 \mu \mathrm{M}$ quercetin was present during the $3 \mathrm{~h}$ exposure of placental explants to hypoxia, from day 3 , the cross-sectional area of the media of chorionic arteries exposed to the CM was significantly lower $(-44 \pm 50 \%, \mathrm{p}<0.030, \mathrm{n}=9)$ compared to the placental HCM condition. After 7 days exposure to the placental CM, the $3 \mu \mathrm{M}$ and $10 \mu \mathrm{M}$ and $20 \mu \mathrm{M}$ RRR- $\alpha$-tocopherol condition resulted in a significant smaller cross-sectional area of the media $(-43 \pm 44 \%, \mathrm{p}<0.032, \mathrm{n}=12,-64 \pm 56 \%, \mathrm{p}<0.041, \mathrm{n}=8$ and $-44 \pm 45 \%$, $\mathrm{p}<0.004, \mathrm{n}=15$, respectively), compared to the placental HCM condition (Figure 4). No changes in cross-sectional area of the lumen and adventitia were found after exposure to placental CM between the placental CCM and HCM condition (Supplementary Figure 1).
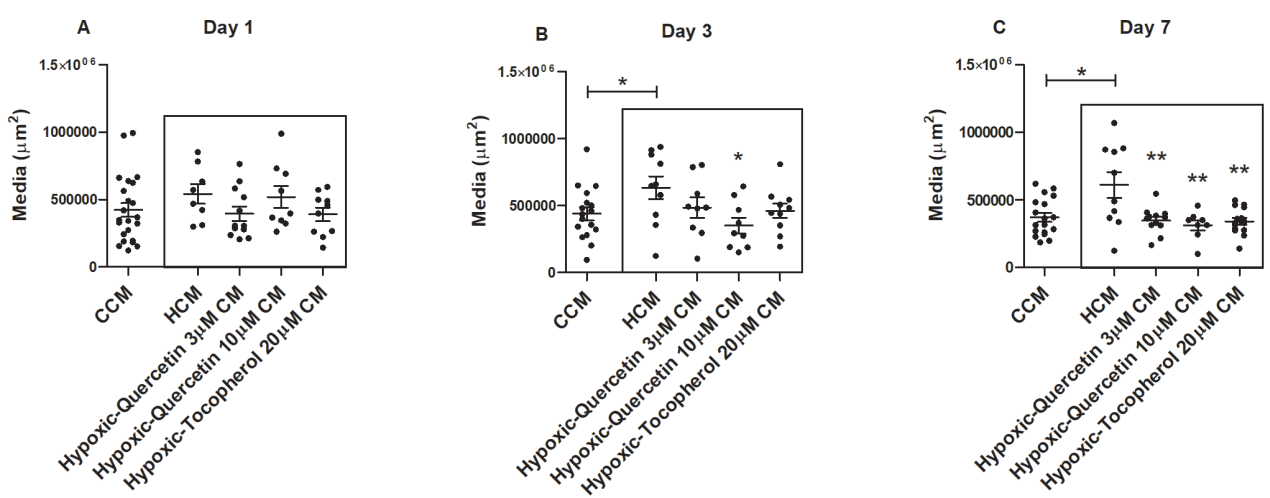

Figure 4. Placental HCM increases vascular cross-sectional area of the media. Cross-sectional area of the media assessed in placental chorionic arteries after 1, 3 or 7 days exposure to placental CCM, $\mathrm{HCM}$, hypoxic-quercetin ( 3 or $10 \mu \mathrm{M})$ or tocopherol $(20 \mu \mathrm{M}) \mathrm{CM}$. Data are expressed as $\mu \mathrm{m} 2$ and are presented as mean with SEM. Conditions in the black square are compared to the HCM condition. **p $\leq$ 0.01. CCM: Control-conditioned medium, CM: Conditioned medium and HCM: Hypoxic-conditioned medium. 


\section{Placental HCM increases vascular proliferation}

To determine whether the increased cross-sectional area of the media after exposure to placental HCM was a result of hyperplasia, vascular proliferation was assessed by KI-67 staining. As presented in figure 5, after 1, 3 and 7 days exposure to placental $\mathrm{HCM}$, increased proliferating nuclei were found $(+130 \pm 110 \%, \mathrm{p}=0.010, \mathrm{n}=10,131$ $\pm 71 \%, \mathrm{p}=0.005, \mathrm{n}=11$ and $+114 \pm 79 \%, \mathrm{p}<0.001, \mathrm{n}=13$, respectively) compared to the placental CCM condition ( $n=27, n=17$ and $n=30$, respectively) (Figure 5). Furthermore, when quercetin $(3 \mu \mathrm{M})$ and RRR- $\alpha$-tocopherol $(20 \mu \mathrm{M})$ was present during the $3 \mathrm{~h}$ placental exposure to hypoxia, after 1 day exposure, the resulting CM significantly reduced proliferating nuclei $(-42 \pm 44 \%, p=0.028, n=14$ and -55 $\pm 48 \%, \mathrm{p}=0.003, \mathrm{n}=15$, respectively), compared to the placental HCM condition. When quercetin $(10 \mu \mathrm{M})$ was present during the $3 \mathrm{~h}$ placental exposure to hypoxia, after 3 days exposure, the resulting CM significantly reduced numbers of proliferating nuclei $(-42 \pm 27 \%, \mathrm{p}=0.027, \mathrm{n}=13)$, compared to the placental HCM condition. After 7 days exposure, all three intervention conditions (quercetin 3 and $10 \mu \mathrm{M}$ and RRR- $\alpha$-tocopherol $20 \mu \mathrm{M})$ reduced numbers of proliferating nuclei $(-43 \pm 38 \%$, $p=$ $0.003, \mathrm{n}=19,-41 \pm 50 \%, \mathrm{p}=0.004, \mathrm{n}=11$ and $-49 \pm 37 \%, \mathrm{p}<0.001, \mathrm{n}=18$, respectively), compared to the placental HCM condition. See supplementary figure 2, for a representative example of the light microscopy images of the Ki-67 immunohistochemistry-stained arterial cross-sections of placental chorionic arteries after 7 days exposure to CCM or HCM.
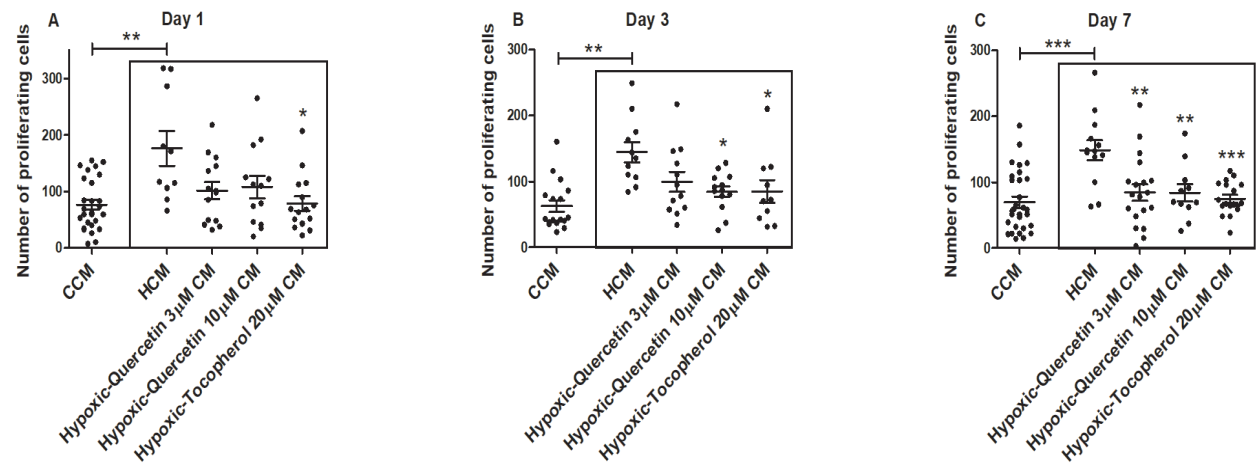

Figure 5. Placental HCM increases vascular proliferation. Proliferating cells assessed in placental chorionic arteries direct after isolation (acute) or after 1, 3 or 7 days exposure to placental CCM, HCM, hypoxic-quercetin $(3$ or $10 \mu \mathrm{M})$ or tocopherol $(20 \mu \mathrm{M}) \mathrm{CM}$. Data are expressed as number of proliferating cells and are presented as mean with SEM. Conditions in the black square are compared to the HCM condition. ${ }^{*} \mathrm{p} \leq 0.05,{ }^{* *} \mathrm{p} \leq 0.01$ and $* * * \mathrm{p} \leq 0.001$. CCM: Control-conditioned medium, CM: Conditioned medium and HCM: Hypoxic-conditioned medium. 


\section{Placental HCM increases vascular contractile responsiveness to U46619}

Ultimately, it was tested if the increased cross-sectional area found in placental chorionic arteries exposed to placental HCM resulted in a different vascular contractile responsiveness to U46619. No differences were found for the LogEC50 calculated from the concentration-dependent contraction curves for U46619 (0.1 nM - 1 $\mu \mathrm{M})$ between the different conditions for each day (Supplementary Figure 3). U46619 (1 $\mu \mathrm{M})$, however, induced a significant increased vascular contraction $(+34 \pm 20 \%, \mathrm{p}=$ 0.003, $\mathrm{n}=10$ ) in placental chorionic arteries exposed to placental HCM for 3 days, compared to the placental CCM condition $(n=21)$. Furthermore, after 3 days exposure, the placental CM, which was produced in the presence of quercetin 3 or $10 \mu \mathrm{M}$ induced a significant lower vascular contraction against U46619 $(1 \mu \mathrm{M})(-30 \pm 22 \%$, $\mathrm{p}=0.018, \mathrm{n}=11$ and $-35 \pm 31 \%, \mathrm{p}=0.016, \mathrm{n}=10$, respectively), compared to the placental HCM condition ( $n=10$ ) (Figure 6). Placental CM did not affect the pEC50 of the concentration-dependent relaxation curves for the NO donor sodium nitroprusside $(0.01 \mathrm{nM}-30 \mu \mathrm{M})$ pre-contracted by U46619 (30 nM) between the conditions (Supplementary Figure 4).
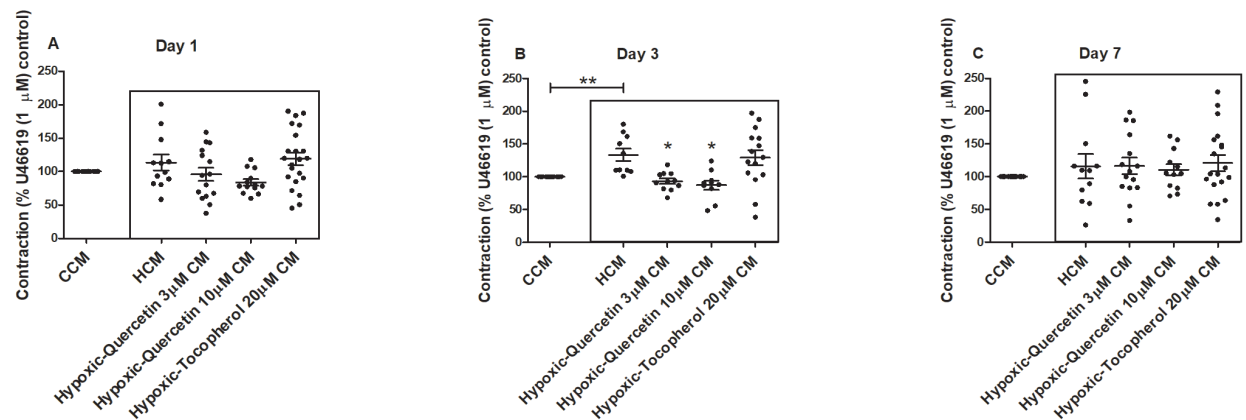

Figure 6. Placental HCM increases vascular contractile responsiveness to U46619. Vascular contractility against $\mathrm{U} 46619(1 \mu \mathrm{M})$ after 1, 3 or 7 days exposure to placental CCM, HCM, hypoxic-quercetin $(3$ or $10 \mu \mathrm{M})$ or tocopherol $(20 \mu \mathrm{M}) \mathrm{CM}$ in placental chorionic arteries. Data are expressed as percentage compared to the vascular contraction in response to $\mathrm{U} 46619(1 \mu \mathrm{M})$ in the control condition and are presented as mean with SEM. Conditions in the black square are compared to the HCM condition. ${ }^{*} \mathrm{p} \leq 0.05$ and ${ }^{* *} \mathrm{p} \leq 0.01$. CCM: Control-conditioned medium, CM: Conditioned medium and HCM: Hypoxic-conditioned medium. 


\section{Discussion}

In the current study, we show for the first time, that intraluminal administration of placental HCM induces vasoconstriction and increased endothelial permeability for $\mathrm{KCl}$ in chorionic arteries. When the most abundant dietary flavonoid quercetin was present during the placental exposure to hypoxia, these effects could be concentration-dependently prevented. Moreover, we show that exposure of cultured EA.hy926 endothelial cells to placental or BeWo HCM decreases cell viability and increases endothelial production of ROS and pro-inflammatory cytokines IL-6 and IL-8. Furthermore, we observed that the cross-sectional area of the arterial media was increased after 3 days of exposure to placental HCM, which was associated with increased vascular proliferation and could be concentration-dependently prevented by the presence of quercetin as well as RRR- $\alpha$-tocopherol during the placental exposure to hypoxia. Besides the effect of placental HCM on vascular contraction and morphology, vascular contractile responsiveness to U46619 was increased after 3 days of exposure, which also could be prevented by the presence of quercetin during the placental exposure to hypoxia.

In PE pathophysiology, impaired uterine spiral artery remodeling by extravillous trophoblast cells is believed to lead to persistently hypoxic, dysfunctional placentae that release pathogenic molecules into the maternal circulation (27). It has been proposed that in response to oxygenation shortage, placental cells release factors that affect the endothelial function in the whole maternal circulation, induce vasoconstriction and increase the contractile responsiveness of the vasculature to vasopressors, which has been demonstrated to persist after delivery (28-30). These mechanisms have been suggested to contribute to an increased risk for cardiovascular disease in later life in women with a history of PE. While several studies support the role of reduction in uteroplacental perfusion pressure in $\mathrm{PE}$, the factors released in response to reduction in uteroplacental perfusion pressure and its contribution in the development of generalized hypertension in PE pathogenesis are the subject of extensive research (10).

In previous work (6), where we mounted freshly isolated chorionic arteries into a wire myograph and used specific receptor antagonists, the AT1 and ET-1 receptors were identified as the main contributors to the acute vasoconstrictive activity of placental HCM. In this study, we show that in chorionic arteries mounted into a pressure myograph, intraluminal administration of placental HCM also induced vascular contraction, suggesting that the contractile response might be endothelium-dependent. Interestingly, angiotensin II type 1 receptor agonistic autoantibody ( $\left.\mathrm{AT}_{1}-\mathrm{AA}\right)$ that are involved in the hypertensive state in PE are known to be induced by placental 
hypoxia and have been implicated as a major contributor to the activation of the AT1 receptor-mediated hypertension in PE (31). Granger et al. already proposed that besides activating the AT1 receptor, increased placental AT 1 -AA secretion upon hypoxia may stimulate endothelial cells to produce ET-1 explaining the significance of their receptors in the development of PE-induced hypertension (32). It is postulated that in severe PE, ET-1 production is increased that much that it loses its paracrine directionality and leads to increased circulating levels and together with $\mathrm{AT}_{1}-\mathrm{AA}$ inducing the local and systemic release of oxidant substances (32-34). Interestingly, administration of ascorbic acid increased the flow-mediated vasodilation in large arteries of former PE women, further demonstrating the role of oxidative stress in maintaining vascular function in PE (35). Supportive to this data, we were able to show that the presence of quercetin during the exposure of placental explants to hypoxia, concentration-dependently protected the vascular contraction induced by placental HCM. Interestingly, plasma levels of antioxidants that counterbalance the increased ROS production were found to be lower in women with PE $(36,37)$ further supporting the role of oxidative stress in the release of vasoactive compounds inducing hypertension in the pathogenesis of PE.

Furthermore, animal studies on the PE phenotype, showed that anti-angiogenic factors like sFlt-1 released by the hypoxic placenta are associated with increased endothelial cell permeability, which is causing proteinuria, cerebral edema and hypertension $(27,31,38)$. Moreover, electron microscopic studies of myometrial vessels and arteries isolated from subcutaneous fat in women with PE have revealed disrupted and enlarged intracellular junctions between endothelial cells (39). In support of this data, we found that vascular permeability for $\mathrm{KCl}$ was increased after intraluminal exposure to placental HCM compared to CCM. In addition, HCM from both placental explants and BeWo cells decreased endothelial cell viability. TNF- $\alpha$, a wellknown elevated pro-inflammatory cytokine in PE, modulates the immune response by increasing vascular permeability, lymphocyte activation, and IL- 6 and IL-8 production upon hypoxic placental stress (40-42). Consequently, these cytokines cause increased endothelial permeability by inducing the expression of adhesion molecules e.g. E-selectin, intercellular adhesion molecule 1 (ICAM1) and vascular cell adhesion molecule 1 (VCAM1) $(1,43)$. Furthermore, these cytokines are involved in the downregulation of endothelial nitric oxide synthase (eNOS), increased $\mathrm{AT}_{1}$ AA production via e.g. T helper and B lymphocyte activation 44, and mitochondrial biogenesis all leading to mitochondrial dysfunction resulting in oxidative stress and increased ROS production $(4,45)$. We found increased IL- 6 and IL-8 cytokine expression and ROS production in endothelial cells exposed to HCM from both placental explants and BeWo cells. In accordance with the effect of placental HCM 
on EA.hy926 endothelial cells found in our study, exposing endothelial cells to serum from women suffering from $\mathrm{PE}$ has been shown to induce endothelial damage (27). Remarkably, it has been demonstrated that in the earliest passage of cultured endothelial cells derived from women with PE, there is an increased endothelial monolayer permeability that is associated with alterations in the junctional adhesion molecule vascular endothelial cadherin and tight junction protein occludin $(39,46)$. These alterations normalized again when these endothelial cells were continuously cultured in vitro suggesting that the extracellular environment in PE contains factors that diminish endothelial barrier function in a reversible manner (39). Collectively, this may suggest that besides alterations found in endothelial cells upon exposure to placental HCM or blood plasma of women with PE, other vascular cell types are involved in the cardiovascular effects in PE.

By comparing cross-sectional areas of the lumen, media, and adventitia of chorionic arteries exposed to placental HCM for up to 7 days, the cross-sectional area of the media, containing the smooth muscle cells, was increased after 3 days exposure compared to the control. To determine whether the increased cross-sectional area of the media was a result of hyperplasia, vascular proliferation was assessed in the same chorionic arteries, which was already significantly increased after 1 day exposure to placental HCM. Both effects were concentration-dependently avoided by the presence of quercetin or RRR- $\alpha$-tocopherol during the placental exposure to hypoxia. $\mathrm{AT}_{1}-\mathrm{AA}$ in $\mathrm{PE}$ or upon placental hypoxia have not only proved to have a profound contribution to the increased vasoconstriction in PE, but is also known to promote smooth muscle growth upon AT1 receptor activation $(3,47)$. Together with pro-inflammatory cytokines, $\mathrm{AT}_{1}-\mathrm{AA}$ is known to promote the endothelial release of vasoactive factors such as ET-1 and TXA2, which stimulates smooth muscle proliferation furthermore through the ETB and thromboxane receptor respectively $(3,34,45)$. Together with our findings, this may suggest that not only endothelial cells, but alterations in smooth muscle cell morphology and/or function may contribute significantly to cardiovascular problems during PE.

3 days of exposure to placental HCM caused an sustained increase in vascular contractile responsiveness to U46619. This finding is in line with a similar increase in vascular contractility to TXA2, angiotensin II (AngII) and ET-1 of omental arteries observed in women suffering from PE (48). In line with the protective effect on vascular morphology found, adding quercetin during the $3 \mathrm{~h}$ exposure of placental explants to hypoxia also normalized the increased vascular contractile responsiveness to U46619. A meta-analysis indicated a two-fold increase in cardiovascular risk in women with a history of PE (49). Also, infants of preeclamptic mothers have 
higher blood pressure during young adulthood and an increased risk for stroke in later life (2). Furthermore, during pregnancy, the microvasculature of women complicated with PE have a higher response to endothelial-dependent vasodilation (acetylcholine) and show no differences in endothelial-independent vasodilation by SNP compared to non-preeclamptic women (50-52). In line with this study, no changes were found in the effect of smooth muscle dilator sodium nitroprusside on arterial relaxation after exposure to placental HCM up to 7 days.

\section{Limitations and future perspectives}

Although the use of primary cells for this approach could be suggested, the endothelial EA.hy926 cell line was used to minimize senescence processes, morphological and functional changes during the cell-cyles and to maximize potential for self-renewal of the cells. By preventing genetic variation among donors, the conditioned medium was tested under similar and stable culture conditions. Placental villous explants were exposed to hypoxia for up to $3 \mathrm{~h}$ since previous studies using placental villous explants ex vivo showed that viability of placental tissue in culture could only be maintained for $4 \mathrm{~h}$ (53). Longer exposure times have been shown to lead to pronounced tissue deterioration and substantial apoptosis of trophoblast cells in placental villous explants, indicating that this model it is only suitable to study acute effects in culture (54). To study the effects of long-term exposure to hypoxia and to determine if placental conditioned-induced changes are trophoblast-specific, the BeWo cell line was used. As shown in figure 6, placental HCM only showed a significant increase in vascular contractile responsiveness to U46619 at day 3, indicating that this parameter is less preserved over time compared to the effect found for the related condition on cross-sectional area of the media and vascular proliferation in chorionic arteries. This finding together with the variation within the test conditions, might be an explanation for the controversial effect of the intervention and underlines the need for alternative assessment options to examine long-term functional changes related to vascular responsiveness.

To investigate the long term effects of placental HCM on vascular morphology, after exposing chorionic arteries, HCM could be replaced by placental CCM to investigate the recovery potential of the arteries. Furthermore, it should be of great interest to compare the proportion of the cross-sectional areal media in resistance blood vessels like omental blood vessels in women with PE compared to controls. Even though atmospheric $21 \% \mathrm{O}_{2}$ is generally considered as a standard culture condition, determining dissolved $\mathrm{O}_{2}$ levels in culture medium and in cultured tissue remains difficult, since many factor including the volume of the media and metabolic activity of the tissue influence medium $\mathrm{O}_{2}$ levels. As recommended by Trenton et. al., 
we constantly aerated our culture media and thereby improved equilibration time, stabilized pericellular oxygen tensions and prevened gradual oxygen diffusion (55). To improve $\mathrm{O}_{2}$ monitoring during culture, we recommend to measure $\mathrm{O}_{2}$ levels in the medium throughout the experiment, and to assess oxygen dependent enzymes including the HIF-PHDs, which are well known as the "oxygen sensors" in placental explants after culturing. For a better physiological approach, our experimental setting can now serve as a tool where the cell lines used in our study can be replaced by freshly isolated trophoblast and HUVEC cells and the effect of blood plasma of $\mathrm{PE}$ women on alterations in vascular function, morphology and endothelial barrier integrity can be examined.

\section{Conclusion}

This study shows that intraluminal administration of placental HCM induces vascular contraction and increases endothelial permeability. It also demonstrates that these placental factors induce endothelial cell death, ROS formation and inflammation, which are all known to be involved in the formation of vasoactive compounds like ET-1 and thus indirectly contributing to vascular contraction. The increased contractility, cross-sectional area and vascular proliferation of the media observed in chorionic arteries upon exposure to placental HCM implies that vascular smooth muscle cells undergo long-lasting adaptations, which may contribute to the cardiovascular effects associated with PE. A better understanding of the complex interplay between the stressed placenta in PE and the maternal cardiovascular system may help in the identification of new serum biomarkers for the prediction of PE and in the design of new diagnostic approaches for a better clinical management. Our work forms a first step to a better knowledge of the long-lasting maternal vascular consequences opening new postpartum treatment and follow-up strategies.

\section{Acknowledgments}

This study was supported by NUTRIM Graduate Program. There was no additional external funding received for this study. 


\section{References}

1. Zarate A, Saucedo R, Valencia J, Manuel L, Hernandez M. Early disturbed placental ischemia and hypoxia creates immune alteration and vascular disorder causing preeclampsia. Arch Med Res. 2014;45(7):519-524.

2. Burton GJ, Redman CW, Roberts JM, Moffett A. Pre-eclampsia: pathophysiology and clinical implications. BMJ. 2019;366:12381.

3. Yu W, Gao W, Rong D, Wu Z, Khalil RA. Molecular determinants of microvascular dysfunction in hypertensive pregnancy and preeclampsia. Microcirculation. 2018(e-pub ahead of print 2018/10/20;doi:10.1111/micc.12508):e12508.

4. Sánchez-Aranguren LC, Prada CE, Riaño-Medina CE, Lopez M. Endothelial dysfunction and preeclampsia: role of oxidative stress. Frontiers in physiology. 2014;5:372.

5. Vangrieken P, Vanterpool SF, van Schooten FJ, Al-Nasiry S, Andriessen P, Degreef $\mathrm{E}$, et al. Histological villous maturation in placentas of complicated pregnancies. Histol Histopathol. 2020; doi:10.14670/HH-18-205):18205.

6. Vangrieken P, Al-Nasiry S, Janssen GMJ, Weseler AR, Spaanderman ME, Bast A, et al. The direct and sustained consequences of severe placental hypoxia on vascular contractility. PLoS One. 2018;13(8):e0202648.

7. Sahay AS, Patil VV, Sundrani DP, Joshi AA, Wagh GN, Gupte SA, et al. A longitudinal study of circulating angiogenic and antiangiogenic factors and AT1-AA levels in preeclampsia. Hypertension Research. 2014;37(8):753.

8. Brennan LJ, Morton JS, Davidge ST. Vascular dysfunction in preeclampsia. Microcirculation. 2014;21(1):4-14.

9. Leik CE, Walsh SW. Neutrophils infiltrate resistance-sized vessels of subcutaneous fat in women with preeclampsia. Hypertension. 2004;44(1):72-77.

10. Shah DA, Khalil RA. Bioactive factors in uteroplacental and systemic circulation link placental ischemia to generalized vascular dysfunction in hypertensive pregnancy and preeclampsia. Biochem Pharmacol. 2015;95(4):211-226.

11. Crews JK, Herrington JN, Granger JP, Khalil RA. Decreased endothelium-dependent vascular relaxation during reduction of uterine perfusion pressure in pregnant rat. Hypertension. 2000;35(1 Pt 2):367-372.

12. Gilbert JS, Babcock SA, Granger JP. Hypertension produced by reduced uterine perfusion in pregnant rats is associated with increased soluble fms-like tyrosine kinase-1 expression. Hypertension. 2007;50(6):1142-1147.

13. Fushima T, Sekimoto A, Minato T, Ito T, Oe Y, Kisu K, et al. Reduced Uterine Perfusion Pressure (RUPP) Model of Preeclampsia in Mice. PLoS One. 2016;11(5):e0155426.

14. Rigotti A. Absorption, transport, and tissue delivery of vitamin E. Molecular 
aspects of medicine. 2007;28(5-6):423-436.

15. Şimşek M, Naziroğlu M, Şimşek H, Cay M, Aksakal M, Kumru S. Blood plasma levels of lipoperoxides, glutathione peroxidase, beta carotene, vitamin A and $\mathrm{E}$ in women with habitual abortion. Cell Biochemistry and Function: Cellular biochemistry and its modulation by active agents or disease. 1998;16(4):227-231.

16. Mesdaghinia E, Mohammad-Ebrahimi B, Foroozanfard F, Banafshe HR. The effect of vitamin $\mathrm{E}$ and aspirin on the uterine artery blood flow in women with recurrent abortion: A single-blind randomized controlled trial. International Journal of Reproductive BioMedicine. 2017;15(10):635.

17. Vogiatzoglou A, Mulligan AA, Lentjes MA, Luben RN, Spencer JP, Schroeter $\mathrm{H}$, et al. Flavonoid intake in European adults (18 to 64 years). PloS one. $2015 ; 10(5)$.

18. Kozłowska A, Szostak-Węgierek D. Flavonoids-food sources, health benefits, and mechanisms involved. Bioactive Molecules in Food Reference Series in Phytochemistry Springer, Cham. 2018.).

19. Debier C. Vitamin E during pre-and postnatal periods. Vitamins \& Hormones. 2007;76:357-373.

20. McDonald SD, Malinowski A, Zhou Q, Yusuf S, Devereaux PJ. Cardiovascular sequelae of preeclampsia/eclampsia: a systematic review and meta-analyses. Am Heart J. 2008;156(5):918-930.

21. Brennan L, Morton JS, Quon A, Davidge ST. Postpartum Vascular Dysfunction in the Reduced Uteroplacental Perfusion Model of Preeclampsia. PLoS One. 2016;11(9):e0162487.

22. Ramsay JE, Stewart F, Greer IA, Sattar N. Microvascular dysfunction: a link between pre-eclampsia and maternal coronary heart disease. BJOG. 2003;110(11):1029-1031.

23. Chambers JC, Fusi L, Malik IS, Haskard DO, De Swiet M, Kooner JS. Association of maternal endothelial dysfunction with preeclampsia. JAMA. 2001;285(12):1607-1612.

24. Wang Y, Zhao S. Vasoactivators and placental vasoactivity. Morgan \& Claypool Life Sciences, San Rafael. 2010.).

25. Boots AW, Veith C, Albrecht C, Bartholome R, Drittij M-J, Claessen SM, et al. The dietary antioxidant quercetin reduces hallmarks of bleomycin-induced lung fibrogenesis in mice. BMC Pulmonary Medicine. 2020;20:1-16.

26. Yu W, Jia L, Park SK, Li J, Gopalan A, Simmons-Menchaca M, et al. Anticancer actions of natural and synthetic vitamin $\mathrm{E}$ forms: RRR- $\alpha$-tocopherol blocks the anticancer actions of $\gamma$-tocopherol. Molecular nutrition \& food research. 2009;53(12):1573-1581.

27. Sava RI, March KL, Pepine CJ. Hypertension in pregnancy: Taking cues 
from pathophysiology for clinical practice. Clin Cardiol. 2018;41(2):220-227.

28. LaMarca B, Amaral LM, Harmon AC, Cornelius DC, Faulkner JL, Cunningham MW, Jr. Placental Ischemia and Resultant Phenotype in Animal Models of Preeclampsia. Curr Hypertens Rep. 2016;18(5):38.

29. Roberts JM, Cooper DW. Pathogenesis and genetics of pre-eclampsia. Lancet. 2001;357(9249):53-56.

30. Henriques AC, Carvalho FH, Feitosa HN, Macena RH, Mota RM, Alencar JC. Endothelial dysfunction after pregnancy-induced hypertension. Int J Gynaecol Obstet. 2014;124(3):230-234.

31. Palei AC, Spradley FT, Warrington JP, George EM, Granger JP. Pathophysiology of hypertension in pre-eclampsia: a lesson in integrative physiology. Acta Physiol (Oxf). 2013;208(3):224-233.

32. George EM, Granger JP. Endothelin: key mediator of hypertension in preeclampsia. Am J Hypertens. 2011;24(9):964-969.

33. Fiore G, Florio P, Micheli L, Nencini C, Rossi M, Cerretani D, et al. Endothelin-1 triggers placental oxidative stress pathways: putative role in preeclampsia. The Journal of Clinical Endocrinology \& Metabolism. 2005;90(7):4205-4210.

34. Dong F, Zhang X, Wold LE, Ren Q, Zhang Z, Ren J. Endothelin-1 enhances oxidative stress, cell proliferation and reduces apoptosis in human umbilical vein endothelial cells: role of ETB receptor, NADPH oxidase and caveolin-1. Br J Pharmacol. 2005;145(3):323-333.

35. Echeverria C, Eltit F, Santibanez JF, Gatica S, Cabello-Verrugio C, Simon F. Endothelial dysfunction in pregnancy metabolic disorders. Biochim Biophys Acta Mol Basis Dis. 2020;1866(2):165414.

36. Nakamura M, Sekizawa A, Purwosunu Y, Okazaki S, Farina A, Wibowo N, et al. Cellular mRNA expressions of anti-oxidant factors in the blood of preeclamptic women. Prenatal Diagnosis: Published in Affiliation With the International Society for Prenatal Diagnosis. 2009;29(7):691-696.

37. Cohen JM, Kramer MS, Platt RW, Basso O, Evans RW, Kahn SR. The association between maternal antioxidant levels in midpregnancy and preeclampsia. Am J Obstet Gynecol. 2015;213(5):695 e691-613.

38. Powe CE, Levine RJ, Karumanchi SA. Preeclampsia, a disease of the maternal endothelium: the role of antiangiogenic factors and implications for later cardiovascular disease. Circulation. 2011;123(24):2856-2869.

39. Wang Y, Lewis DF, Gu Y, Zhang Y, Alexander JS, Granger DN. Placental trophoblast-derived factors diminish endothelial barrier function. J Clin Endocrinol Metab. 2004;89(5):2421-2428.

40. LaMarca B, Speed J, Fournier L, Babcock SA, Berry H, Cockrell K, et al. Hypertension in response to chronic reductions in uterine perfusion in pregnant rats: 
effect of tumor necrosis factor-alpha blockade. Hypertension. 2008;52(6):11611167.

41. Matthiesen L, Berg G, Ernerudh J, Ekerfelt C, Jonsson Y, Sharma S. Immunology of preeclampsia. Chem Immunol Allergy. 2005;89:49-61.

42. Possomato-Vieira JS, Khalil RA. Mechanisms of Endothelial Dysfunction in Hypertensive Pregnancy and Preeclampsia. Adv Pharmacol. 2016;77:361-431.

43. Lockwood CJ, Yen CF, Basar M, Kayisli UA, Martel M, Buhimschi I, et al. Preeclampsia-related inflammatory cytokines regulate interleukin-6 expression in human decidual cells. Am J Pathol. 2008;172(6):1571-1579.

44. LaMarca B, Brewer J, Wallace K. IL-6-induced pathophysiology during pre-eclampsia: potential therapeutic role for magnesium sulfate? International journal of interferon, cytokine and mediator research: IJIM. 2011;2011(3):59.

45. Majed BH, Khalil RA. Molecular mechanisms regulating the vascular prostacyclin pathways and their adaptation during pregnancy and in the newborn. Pharmacological reviews. 2012;64(3):540-582.

46. Wang Y, Gu Y, Granger DN, Roberts JM, Alexander JS. Endothelial junctional protein redistribution and increased monolayer permeability in human umbilical vein endothelial cells isolated during preeclampsia. Am J Obstet Gynecol. 2002;186(2):214-220.

47. Jia G, Aroor AR, Meininger GA, Sowers JR. Glucose, Insulin and Potential Strategies of Vascular Stiffening. In: Blood Pressure and Arterial Wall Mechanics in Cardiovascular Diseases.) Springer2014, 423-434.

48. Belfort MA, Saade GR, Suresh M, Kramer W, Vedernikov YP. Effects of selected vasoconstrictor agonists on isolated omental artery from premenopausal nonpregnant women and from normal and preeclamptic pregnant women. Am J Obstet Gynecol. 1996;174(2):687-693.

49. Bellamy L, Casas JP, Hingorani AD, Williams DJ. Pre-eclampsia and risk of cardiovascular disease and cancer in later life: systematic review and meta-analysis. BMJ. 2007;335(7627):974.

50. Brandao AH, Felix LR, Patricio Edo C, Leite HV, Cabral AC. Difference of endothelial function during pregnancies as a method to predict preeclampsia. Arch Gynecol Obstet. 2014;290(3):471-477.

51. Murphy MS, Vignarajah M, Smith GN. Increased microvascular vasodilation and cardiovascular risk following a pre-eclamptic pregnancy. Physiol Rep. 2014;2(11).

52. Davis KR, Ponnampalam J, Hayman R, Baker PN, Arulkumaran S, Donnelly R. Microvascular vasodilator response to acetylcholine is increased in women with pre-eclampsia. BJOG. 2001;108(6):610-614.

53. Sooranna SR, Oteng-Ntim E, Meah R, Ryder TA, Bajoria R. Characteri- 
zation of human placental explants: morphological, biochemical and physiological studies using first and third trimester placenta. Hum Reprod. 1999;14(2):536-541.

54. Di Santo S, Malek A, Sager R, Andres AC, Schneider H. Trophoblast viability in perfused term placental tissue and explant cultures limited to 7-24 hours. Placenta. 2003;24(8-9):882-894.

55. Place TL, Domann FE, Case AJ. Limitations of oxygen delivery to cells in culture: An underappreciated problem in basic and translational research. Free Radic Biol Med. 2017;113:311-322. 


\section{Supplementary Figures}

To determine if conditioned medium from placental explants exposed to hypoxia affects arterial cross-sectional area, placental chorionic arteries were exposed to placental conditioned medium up to 7 days after which the cross-sectional area of the lumen, media and adventitia were assessed. Cross sectional area of the lumen and adventitia was not affected by hypoxic-conditioned placental (HCM) medium compared to the control condition.
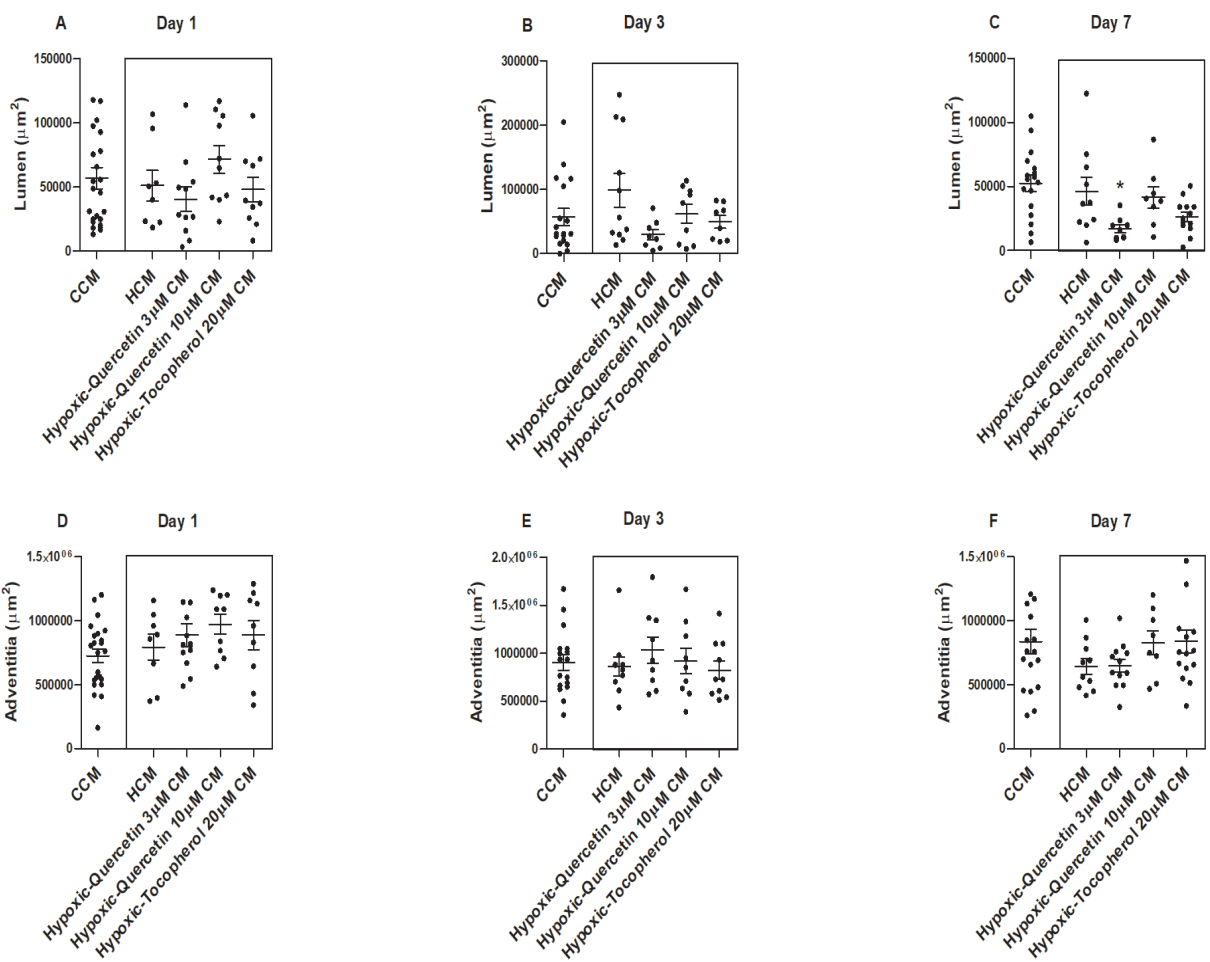

Supplementary Figure 1: No changes in cross sectional area of the lumen and adventitia after exposure to conditioned media between the different conditions. Cross-sectional area of the lumen and adventitia assessed in placental chorionic arteries direct after isolation (acute) or after 1, 3 or 7 days exposure to placental CCM, HCM, hypoxic-quercetin $(3$ or $10 \mu \mathrm{M})$ or tocopherol $(20 \mu \mathrm{M}) \mathrm{CM}$. Data are expressed as $\mu \mathrm{m} 2$ and are presented as mean with SEM. Conditions in the black square are compared to the HCM condition. ${ }^{* *} \mathrm{p} \leq 0.01$. CCM: Control-conditioned medium, CM: Conditioned medium and HCM: Hypoxic-conditioned medium. 
To determine whether the increased cross-sectional area of the media after exposure to placental HCM was a result of hyperplasia, vascular proliferation was assessed by KI-67 staining.

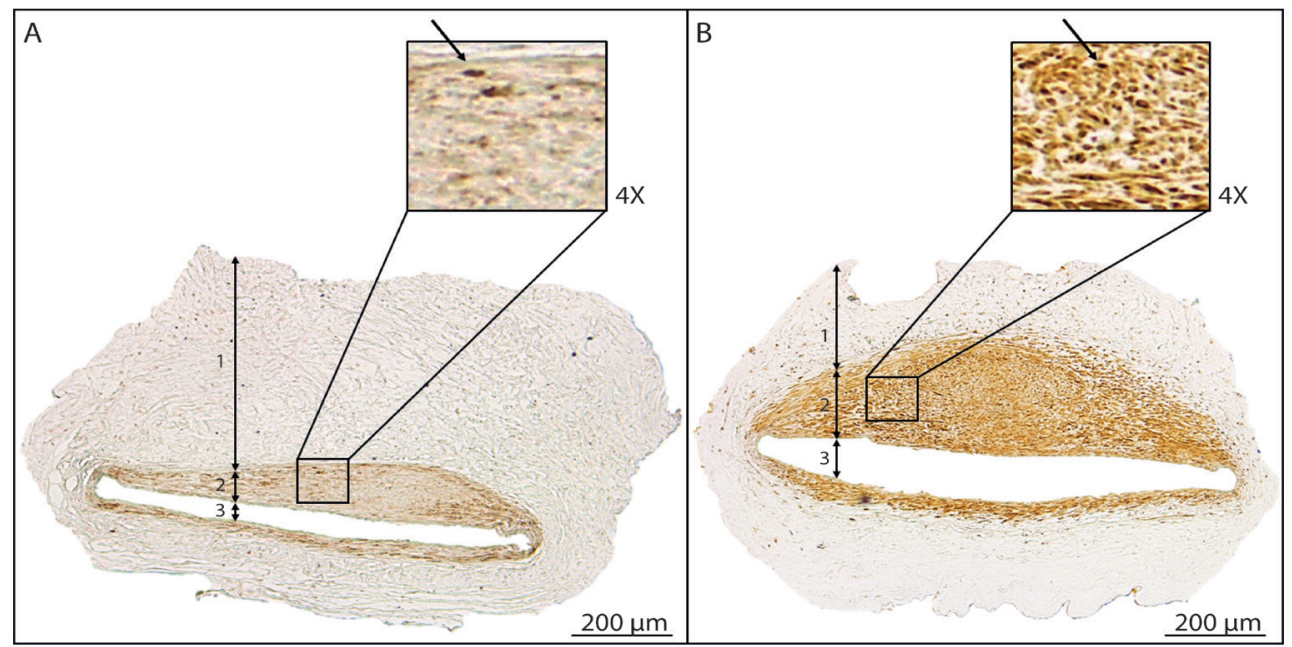

Supplementary Figure 2: Representative example of the light microscopy images of the Ki-67 immunohistochemistry-stained arterial cross-sections of placental chorionic arteries after $\mathbf{7}$ days exposure to CCM or HCM. The arterial adventitia (1), arterial media (2) and arterial adventitia (3). For each condition, the singular arrow indicates a proliferating nucleus. CCM: Control-conditioned medium and HCM: Hypoxic-conditioned medium.

It was tested if the increased cross-sectional area found in placental chorionic arteries exposed to placental HCM resulted in a different vascular contractile responsiveness to U46619.
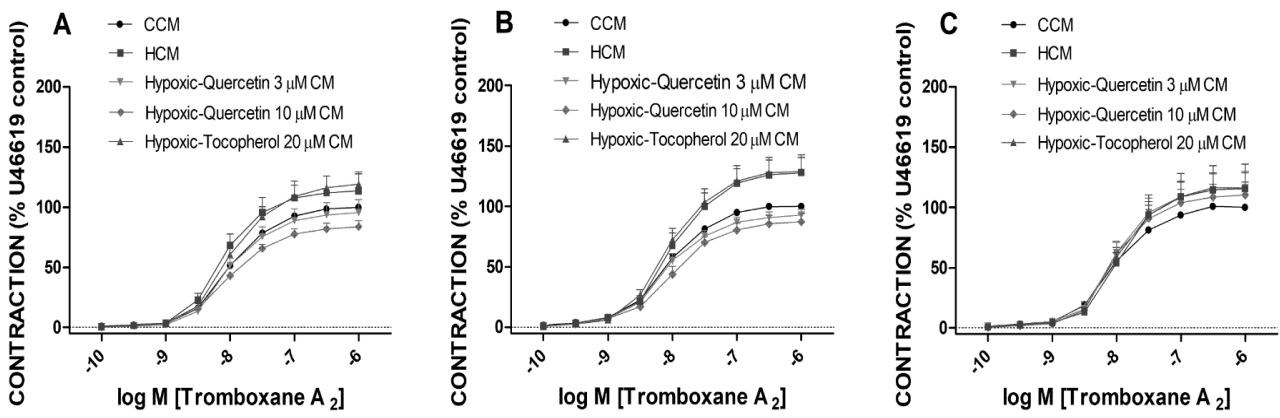

Supplementary Figure 3: Placental HCM increases vascular contractile responsiveness to U46619. Concentration-dependent contractions of chorionic arteries induced by U46619 $(0.1 \mathrm{nM}-1 \mu \mathrm{M})$ after 1,3 or 7 days exposure to placental CCM, HCM, hypoxic-quercetin $(3$ or $10 \mu \mathrm{M})$ or tocopherol $(20$ $\mu \mathrm{M}) \mathrm{CM}$. Data are expressed as percentage compared to the vascular contraction in response to U46619 in the control condition and are presented as mean with SEM. CCM: Control-conditioned medium, $\mathrm{CM}$ : Conditioned medium and HCM: Hypoxic-conditioned medium. 
Hypoxic-conditioned placental medium did not affect the pEC50 of the concentration-dependent relaxation curves for the NO donor sodium nitroprusside $(0.01 \mathrm{nM}$ $-30 \mu \mathrm{M})$ pre-contracted by $\mathrm{U} 46619(30 \mathrm{nM})$ between the conditions.
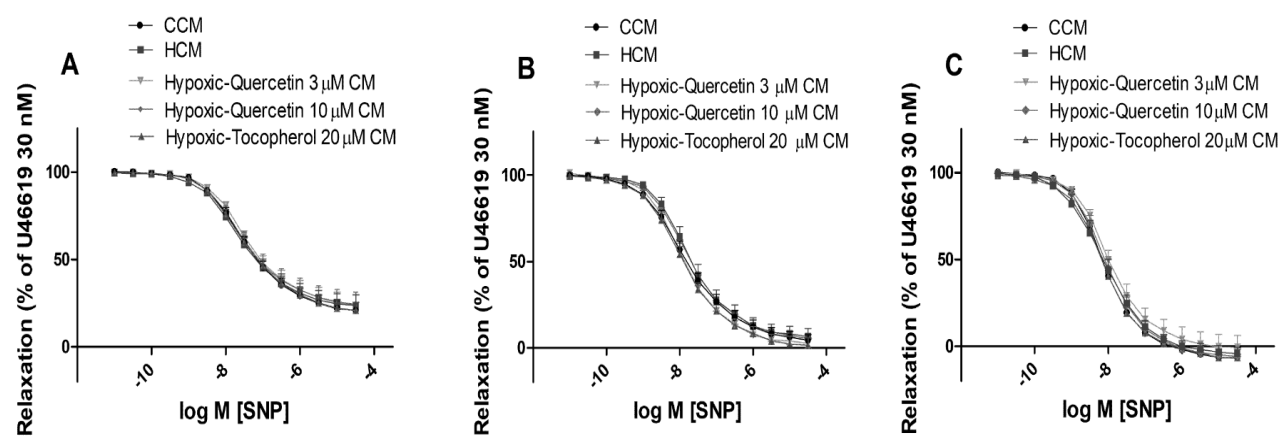

Supplementary Figure 4: Placental CM did not affect the pEC50 of the concentration-dependent relaxation curves for the NO donor sodium nitroprusside. Concentration-dependent relaxation of placental chorionic arteries induced by donor sodium nitroprusside $(0.01 \mathrm{nM}-30 \mu \mathrm{M})$ pre-contracted by $\mathrm{U} 46619(30 \mathrm{nM})$ after 1,3 or 7 days exposure to placental CCM, HCM, hypoxic-quercetin ( 3 or $10 \mu \mathrm{M})$ or tocopherol $(20 \mu \mathrm{M}) \mathrm{CM}$. Data are expressed as percentage compared to the vascular contraction against U46619 $(1 \mu \mathrm{M})$ in the control condition and are presented as mean with SEM. CCM: Control-conditioned medium, CM: Conditioned medium and HCM: Hypoxic-conditioned medium. 


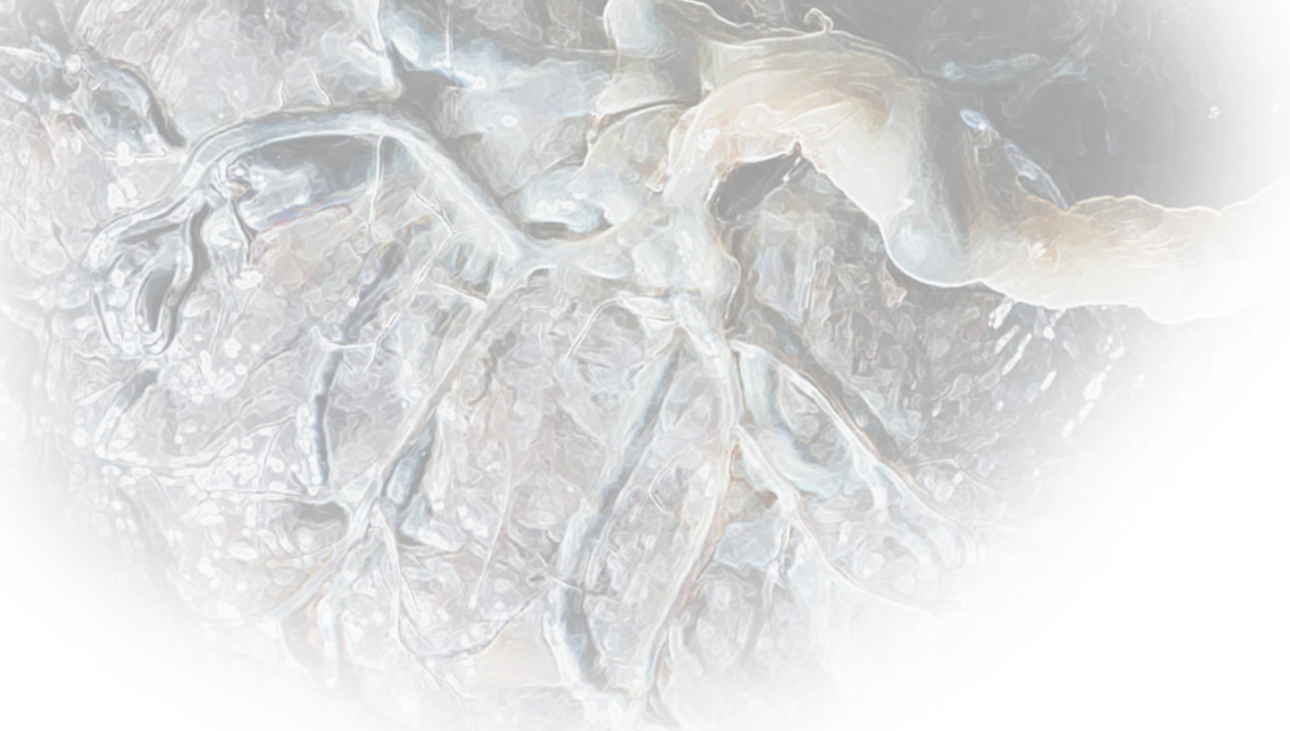




\section{Chapter 7}

General discussion and summary 
This thesis is focusing on how abnormal placental development and function is linked to the pathophysiology of preeclampsia (PE). It provides a set of identified parameters related to placental hyper maturity that can be used to identify more subtypes of PE, which subsequently will help in more directed clinical management and in the prevention of recurrence of PE during a next pregnancy (Chapter 2). Furthermore, this thesis provides new insights into the contribution of mitochondria in mediating placental oxidative stress in PE placentae and upon hypoxia (Chapter 3 and 4). Moreover, we created a better understanding of the interplay between a hypoxic placenta and the vascular system in terms of acute and sustained vascular contractility, morphology and endothelial integrity (Chapter 5 and 6).

\section{Histological adaptations of the placenta in the pathogenesis of $P E$}

$\mathrm{PE}$ is the most common hypertensive disorders of pregnancy occurring in 5-8\% of all pregnancies worldwide. It is the leading cause of morbidity and mortality for pregnant women in the developed world and has a significant economic burden on healthcare systems $(1,2)$. The only present "cure" for PE is delivery of the placenta, often necessitating a premature delivery, with subsequent augmented short-term morbidity and mortality for the newborn (3). PE originates at the placental bed as a result of impaired trophoblast invasion of uterine spiral arteries located in the decidua and myometrium. Trophoblasts (Greek, trophe $=$ nutrition and - blast $=$ a primordial cell) forms the blastocyst outer trophectoderm layer during early development, which will generate all the extra-embryonic trophoblast cell types; cytotrophoblast, syncytiotrophoblast and extra-villous trophoblast cells. Trophoblast cells have an important contribution to adplacentation, implantation and endocrine support of pregnancy and eventually forms the first barrier between the maternal and fetal circulation $(4,5)$. Normally, trophoblastic invasion takes place from 8 to 18 weeks of pregnancy and drives the physiological adaptation process of spiral arteries to ensure a continuous blood flow of maternal blood into the placental intervillous space. Maladaptation of the spiral arteries leads to a persistent high-resistance maternal-placental flow, generally increased vascular resistance, and attenuated blood flow $(6,7)$. These changes, in turn, result in rapidly cycling normoxic and hypoxic states in the placental intervillous space causing ischemia-reperfusion injury, which is characterized by the release of reactive oxygen species (ROS) and a failing antioxidant scavenging capacity. Of the potential ROS sources described to date, xantine oxidases, NADPH oxidase (Nox), mitochondria, and uncoupled nitric oxidase synthase are most likely the main contributors to reperfusion-induced oxidative stress and represents priority targets for therapeutic intervention (8). Although all four enzymatic sources are present in most tissues, priority and emphasis has been given to the mi- 
tochondria that are enriched in metabolically active organs such as the heart and the placenta (8). Increased ROS production and a failing antioxidant system are closely linked to inflammation, one of which can be easily induced by another (9). Furthermore, when the tissue is deprived of adequate oxygen supply, the formation of new blood vessels (angiogenesis) will be promoted. Hypoxia induces vessel growth by upregulating multiple pro-angiogenic pathways that mediate key aspects of stromal, endothelial, and vascular support cell biology. Interestingly, recent studies show that, besides vessel growth, hypoxia influences additional aspects of angiogenesis, including vessel maturation, function, and patterning. Extensive research has proven the integral role of hypoxia and HIF signaling in human diseases such as PE (10-12). For instance, in chronic utero-placental hypoxia, the production of specific angiogenic factors is activated including vascular endothelial growth factor-A (VEGF-A) and its receptors VEGF-R1 and VEGD-R2, which result in increased branching-angiogenesis of fetal capillaries in the placenta (13). Increased branching angiogenesis stimulates branching of the placental villi and results in a compensatory increased exchange surface between the placenta and the maternal blood, which corresponds to the histological image of accelerated villous maturation. In chapter 2, 11 markers of histological villous remodeling demonstrated accelerated villous maturation in PE placentae. Our findings indicated that placentae in PE shift from growth to maturation earlier than placentae in normal pregnancies. The 11 parameters identified in our study as presented in chapter $\mathbf{2}$, not only allow us to study histological adaptations that take place in the placenta complicated with PE, but may also be used to correlate with clinical parameters of the mother and subsequently help in the identification of different clinical subtypes of PE. Advancing our understanding of the pathophysiology underlying PE through the identification of clinically relevant disease subtypes would not only clarify the heterogeneity observed in this disorder, but will also help in the development and/or application of etiology-focused screening tools and therapies for PE. These tools would direct the care of women and their offspring affected by PE towards an etiological-based intervention, which will expand the current symptomatic-focused intervention.

\section{Heterogeneity of PE}

$\mathrm{PE}$ is a heterogeneous syndrome, with a broad spectrum of clinical presentations, affecting multiple maternal organs, placental function and fetal growth $(3,14)$. The common denominator is considered to be defective spiral artery remodeling. Several factors are known to play a role in this defective remodeling including the maternal immune system, the biology of the trophoblasts and trophoblast differentiation. These factors are acting in concert with factors beyond the placenta such as the susceptibility of the maternal cardiovascular system, the action of the macrophage 
defense mechanism and impaired activation/ action of decidua natural killer (NK) cells, which regulates the invasion of extracellular trophoblasts (15). Two distinct subclasses of PE (early- and late-onset PE) are well recognized in literature (16). Although these subclasses show overlap, there is now increasing evidence that they differ in pathophysiology (17-19). Moreover, these two subgroups of PE differ with respect to a variety of biomarkers including the number of angiogenic, antiangiogenic, immunological, and oxidative factors (18). Until now, characterization of early and late-onset PE is only based on the gestational age at diagnosis. Early-onset PE develops before 34 weeks of pregnancy and is generally considered as a feto-placental disorder. This subtype is associated with reduced placental volume and dysfunction, fetal growth restriction, abnormal umbilical and uterine artery Doppler evaluation, and adverse neonatal and maternal outcomes. Late-onset PE occurs after 34 weeks of gestation and is associated as a maternal-vascular disorder. It has less severe outcomes and is mostly associated with normal fetal growth and Doppler evaluation (18). Some investigators have considered that besides early- and late-onset PE there might be separate pathological entities instead of being one fundamental process expressing varying degrees of clinical severity and that the two subtypes of PE represent more than one pathophysiologic process $(20,21)$. This variety of separate pathological entities are yet to be defined and may be an explanation for the failing population-based interventions in women at high risk for developing PE. Allocating all cases to one general PE group may explain why, despite the strong association of PE with oxidative stress, human studies using systematically acting antioxidants like vitamin $\mathrm{C}$ and $\mathrm{E}$ as a treatment for PE were generally unsuccessful (22). Furthermore, the incidence of early-onset $\mathrm{PE}$ is much lower compared to late-onset $\mathrm{PE}$ and together with the differences in clinical outcomes, it is methodologically more difficult to perform a study with sufficient statistical power using separate subgroups of PE. The failing identification of PE subgroups based on its clinical presentation and its following limitations in current PE research underlines the need for a better understanding of the molecular mechanisms involved in the development of placental oxidative stress and subsequent alterations in its secretome, which drives the progression of PE. Improved knowledge in these fundamental mechanisms of its pathogenesis will provide researchers with a tool to allocate patients to specific $\mathrm{PE}$ subgroups and will help in better awareness of the diverse pathological image of PE.

\section{Research and clinical implications of mitochondria in PE and upon placental hypoxia}

It is well-known that disturbed placental perfusion, as observed in patients suffering from PE, can trigger the production of ROS $[9,10]$. Interestingly, oxidative stress is not only known to trigger trophoblast cell death, but also to accelerate tropho- 
blast turnover promoting the release of placental secreted factors into the maternal circulation including shedding of microparticles and vesicles from the syncytiotrophoblast containing vasoactive/ pro-inflammatory substances, and oxidative stress mediators, which subsequently trigger the symptoms of PE (23). Although the presence of placental oxidative stress and its contribution to the PE pathophysiology is commonly acknowledged, the sources of ROS and the exact underlying processes of its formation remain obscure. As illustrated in figure 1, cellular hypoxia as a result of impaired placental perfusion leads to damage of the mitochondrial electron transport chain (ETC) which, as the ETC is the main site for intracellular oxygen consumption in the cell, results in increased production of $\operatorname{ROS}(24,25)$. It is commonly acknowledged that mitochondrial dysfunction causes a reduction in oxygen consumption, increased mitophagy and an increase in superoxide production (26). Although oxidative stress is well-linked to PE pathophysiology, few studies have examined the connection between impaired oxygen supply to the placenta and mitochondrial dysfunction and ROS.

In the current thesis (Chapter 3 and 4), we reported that preeclamptic placentae and placentae or BeWo trophoblasts exposed to hypoxia are characterized by higher levels of oxidized glutathione (GSSG), a higher total antioxidant capacity (Trolox equivalent antioxidant capacity, TEAC) and higher mRNA levels of the mitochondrial-located antioxidant enzyme manganese-dependent superoxide dismutase 2 (MnSOD2) compared to their controls. Considering the increased oxidative stress, elevated mitochondrial-specific antioxidant expression in PE and hypoxia-exposed placenta and the notion that mitochondria are the main intracellular site for cellular $\mathrm{O}_{2}$ consumption, our data suggest that mitochondria are a significant source for ROS. In agreement with this observation, the mitochondrial-targeted antioxidant MitoQ concentration-dependently reduced intracellular ROS levels and proportionally lowered GSSG levels in BeWo cells during hypoxia in our study (Chapter 4). This is in line with previous finding by Aljunaidy et al. (27), who showed not only normalization of oxidative stress levels by MitoQ in a hypoxic rat model of reduced placental perfusion, but also protection against sex- and age-dependent alterations in cardiac and vascular health of offspring later in life (24, 28-30).

Moreover, our data in chapter 3 and 4, showed evidence for significant reductions in mitochondrial content in PE placentae and BeWo trophoblasts exposed to hypoxia compared to controls, which was illustrated by reductions in mtDNA copy number and reduced citrate synthase activity, both well-established markers of mitochondrial content (31). In addition, a recent study using transmission electron microscopy revealed swelling, increased mitochondrial lumen and irregular arrangement 
of mitochondrial cristae in early-onset PE placentae (32). These reductions in mitochondrial content were accompanied by an increased abundance of key constituents of glycolysis and a decrease in the abundance of complex III of the mitochondrial ETC. Several human studies showed significant reductions in adenosine triphosphate (ATP) levels in PE placentae, which is suggestive of impaired mitochondrial functioning (33-35). Our data indicate that in response to PE or hypoxia, placental energy metabolism shifts from oxidative phosphorylation to glycolysis (Figure 1) as measured by gene and protein expression of hexokinase II and enzyme activity of phosphofructokinase. The increased reliance on glycolysis in PE placentae may be a consequence of lower $\mathrm{O}_{2}$ levels, the decreased mitochondrial content and impaired functioning of the ETC. This shift to anaerobic respiration may be a protective response of the placenta to the impaired placental perfusion in $\mathrm{PE}$ and so saving $\mathrm{O}_{2}$ for the growing fetus (36). Moreover, dysfunctional mitochondria are not only generating excessive ROS, but also induce inflammation as well as apoptosis (Figure 1), both of which have been essentially implicated in the pathogenesis of PE (37-39). Increased placental mitochondrial ROS may directly damage mtDNA, inhibit mitochondrial biogenesis and promote mitophagy. In line with the decreased mitochondrial content, mRNA and protein levels of key molecules involved in the regulation of mitochondrial biogenesis were lower in preeclamptic placentae and placentae or BeWo trophoblasts exposed to hypoxia while the abundance of constituents of the mitophagy, autophagy and mitochondrial fission machinery was higher compared to controls.

Our data in chapters 3 and 4 provides valuable new insights into the pathophysiology of PE and more specifically, into how specific mitochondrial abnormalities in PE placentae may well be linked to the development of oxidative stress. These studies thereby add to our understanding of how mitochondrially targeted antioxidants may serve as a better therapeutic target for the treatment of PE. We comprehensively characterized and assessed mitochondrial content and the regulatory pathways controlling mitochondrial content and function in PE placentae. In addition, whether or not mitochondrially targeted strategies have therapeutic potential in the treatment of $\mathrm{PE}$ remains to be discovered in more detail. 


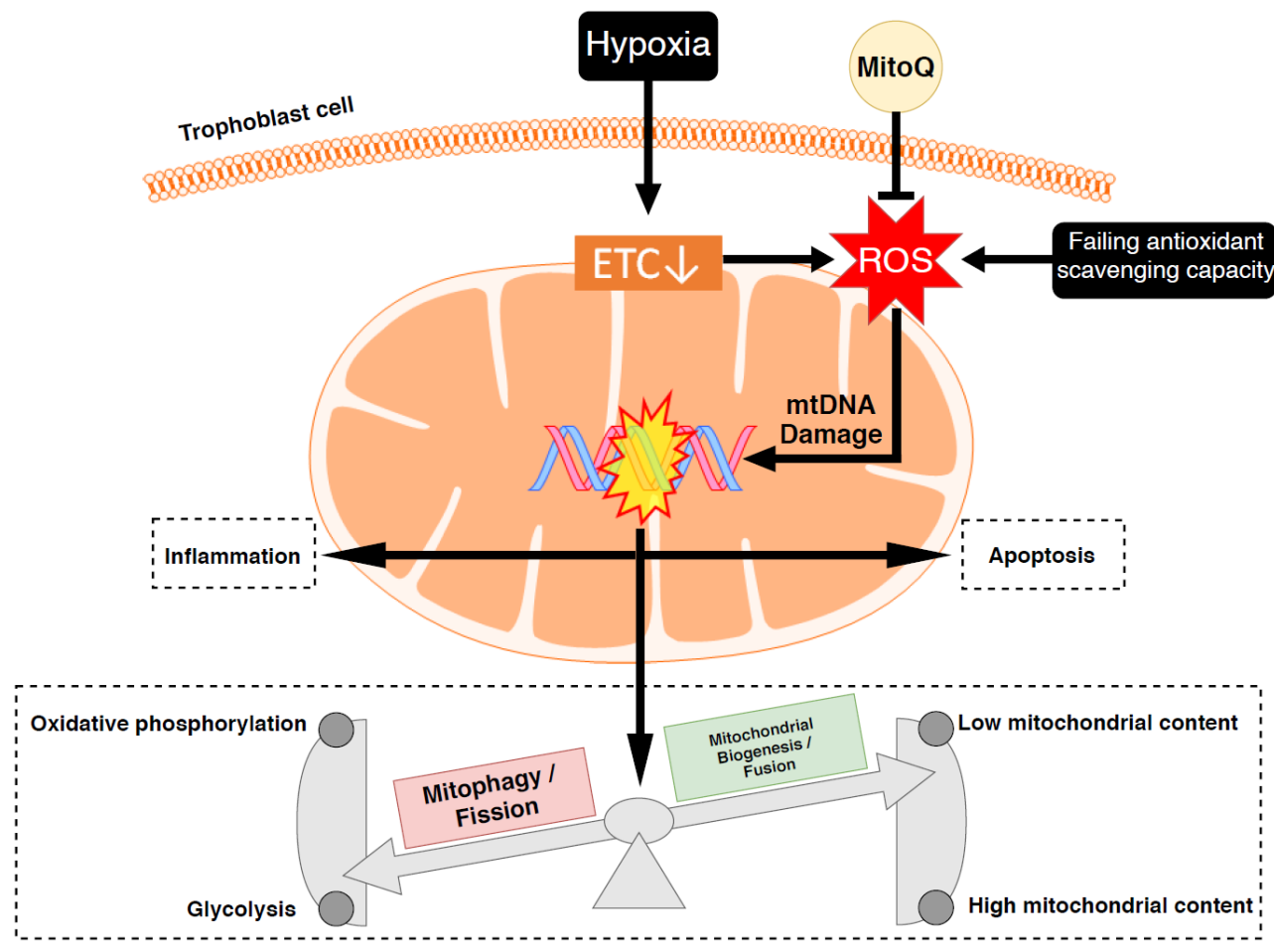

Figure 1. Schematic representation of the proposed sequence of mitochondrial events upon placental hypoxia. Placental hypoxia impairs the function of the mitochondrial electron transport chain, which results in the formation of reactive oxygen species, which can be neutralized by the mitochondrially targeted antioxidant MitoQ. When the antioxidant scavenging capacity is failing, excessive reactive oxygen species accumulation will damage mitochondrial DNA, which promotes inflammation, apoptosis, and mitochondrial fission and subsequent mitophagy. Due to the lower mitochondrial content, aerobic oxidative phosphorylation shifts to anaerobic glycolysis. ETC, Mitochondrial electron transport chain; ROS, Reactive oxygen species.

\section{Future directions in mitochondrial research}

Data in this thesis are the first one that provides a detailed overview of mitochondrial abnormalities and the pathways controlling mitochondrial function and content in $\mathrm{PE}$, which is considered a strength of our study. One obvious limitation, however, is a well-recognized difficulty and is the lack of non-laboured, healthy pre-term control placental samples. The reason for this is that caesarean sections are rarely performed in obstetrically normal pregnancies at gestational age equivalent to those in early-onset PE (Chapter 3). Moreover, as mitophagy is a process evolving during time in fluxes, and therefore the assessed changes in protein and mRNA expression levels in our study are not per se indicative of active or inhibited mitophagy but rather are to be taken as indications of potential changes in the process of mitophagy. The same concept holds true for the process of mitochondrial biogenesis. Besides, we did not 
directly assess mitochondrial function by means of respirometry, which prevents hard conclusions regarding alterations in mitochondrial functionality in PE or upon hypoxia (Chapter 3 and 4). Therefore, future studies including measurements of mitochondrial respiration, and assessment of actual mitophagy and mitochondrial biogenesis including electron microscopy and recently developed assays for measuring mitophagy, e.g. MitoTimer, mt-Keima, and Mito-QC (40), would affirm our conclusion.

Eventually, the specifically identified alterations related to mitochondrial function and oxidative stress may together with the histological adaptations in the placenta found in chapter 2, be linked to the clinical profile of the mother and serve as a tool to efficiently identify more subgroups of PE in larger cohorts. Moving forward, the use of a PE subtype paradigm may allow for more homogeneous patient population selection in study designs, leading to greater scientific contributions in the understanding of PE pathophysiology, the discovery of highly accurate predictive biomarkers, and targeted therapeutic interventions tailored to each disease subtype.

\section{Vascular consequences of and altered placental secretome and its medical implications}

The pathophysiological processes that underlie early-onset PE prior to the alterations in vascular function include defective placentation and apoptosis of invasive cytotrophoblasts causing inadequate remodeling of spiral arteries leading to a decrease in blood flow to the placenta (41-43). Subsequent placental ischemia/hypoxia leading to oxidative stress (42) results in the production of a variety of placental secreted factors (secretome) that collectively have profound effects on blood flow and arterial pressure regulation. In animal studies, reduced uterine perfusion has been shown to subsequently induce hypertension (44-46). In line, data in this thesis (Chapter 5) show that placental hypoxic-conditioned medium (HCM) increase vasoconstriction via the Angiotensin II receptor type 1 (AT1) and (ET-1) receptor and increased vascular contractility to a thromboxane-A2 (TXA2) agonist analogue (U46619) in chorionic arteries mounted in a wire myograph compared to control conditioned medium (CCM) (Figure 2A). When the exposure of placental explants to hypoxia was combined with the flavonoid quercetin, the acute and sustained vascular effects to the resulting placental conditioned medium could be prevented in a concentration-dependent manner (47). Quercetin is a natural pigment present in many fruits, vegetables, and grains and one of the most abundant antioxidants in the diet and may help to reduce inflammation (48). To test whether the observed effects were endothelial-mediated, the same conditions were tested in a pressure myograph (Figure $2 \mathrm{~B}$ ), where the placental conditioned medium could be administrated intraluminally 
(Chapter 6). In line, intraluminal administration of placental HCM also induced vascular contraction, suggesting that the contractile response might be endothelium-dependent, which helps in the selections of new curative strategies.
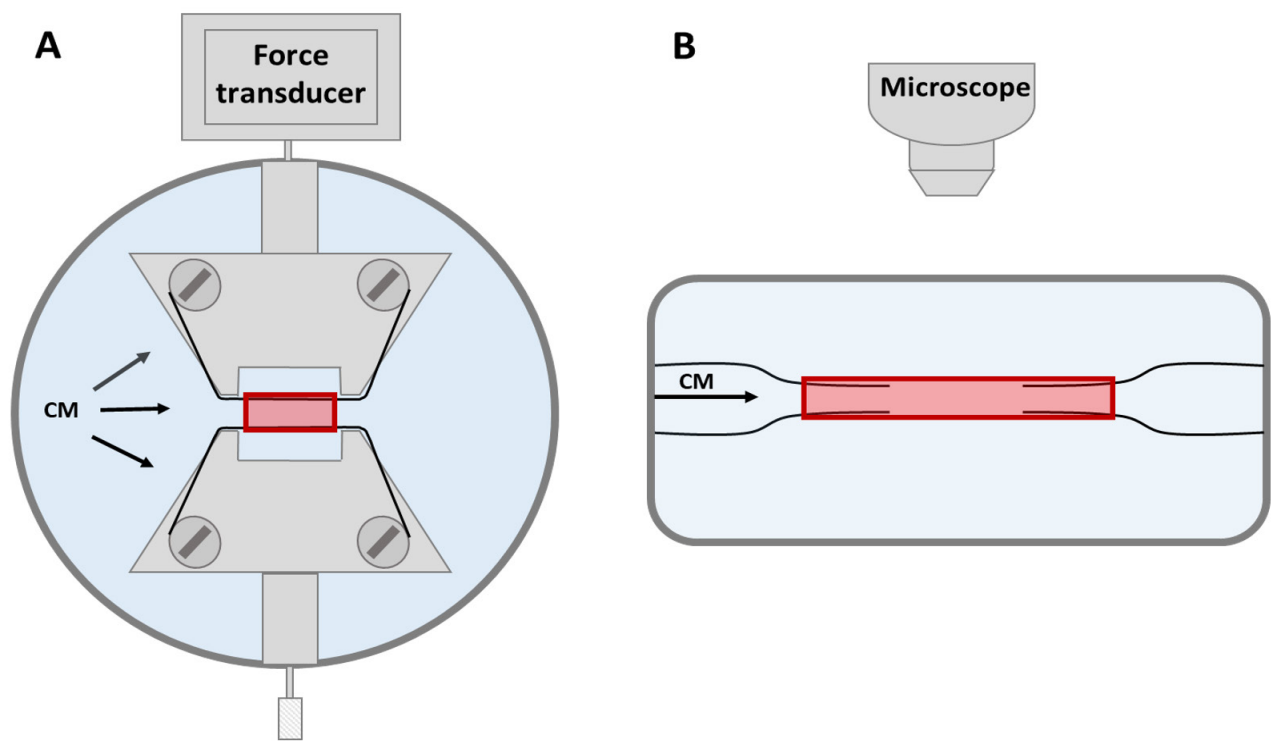

Figure 2. Schematic representation of the pressure wire myograph (A) and pressure myograph (B) setting. For the myograph setting (A), two stainless steel wires were lead through the lumen of the artery (indicated in red) in HEPES buffer and were connected to a displacement device and an isometric force transducer. Contractile responses were expressed as the increase in wall tension (increase in force/ twice the segment length; $\mathrm{N} / \mathrm{m}$ ). For the pressure myograph setting (B), an isolated artery (indicated in red) was mounted onto two glass cannulas in HEPES buffer and connected to a pressure servo controller with a peristaltic pump to ensure a controlled internal pressure and flow. A video dimension analyzer measured the lumen diameter (expressed as delta diameter change $(\mu \mathrm{m}) . \mathrm{CM}$ : conditioned medium.

Animal studies on the PE phenotype and on hypoxic placentae showed that anti-angiogenic factors like soluble fms-like tyrosine kinase-1 (sFlt-1) released by a hypoxic placenta, are associated with increased endothelial cell permeability, which is causing proteinuria, cerebral edema and hypertension (6, 49-52). Moreover, electron microscopic studies of myometrial vessels and arteries isolated from subcutaneous fat in women with PE have revealed disrupted and enlarged intracellular junctions between endothelial cells (53). As schematically represented in figure 3, plasma levels of sFlt-1 are constantly elevated in PE and upregulated in cytotrophoblast upon hypoxia and by sequestering circulating VEGF and placental like growth factor (PLGF) it prevents activation of their receptor, subsequently disturbs normal endothelial function. Consequently, increased levels of soluble endoglin (sEng) block transforming growth factor beta 1 (TGF $\beta 1$ ) signaling, which inhibits vasodilation 
in rats and leads to leaky capillaries (42). In support of this data, we found (Chapter 6) that vascular permeability for potassium chloride $(\mathrm{KCl})$ was increased after intraluminal exposure to placental HCM compared to CCM. In addition, HCM from both placental explants and BeWo cells decreased endothelial cell viability and increased IL- 6 and IL-8 cytokine expression and ROS production in endothelial cells. Tumor necrosis factor alpha (TNF- $\alpha$ ), a well-known elevated pro-inflammatory cytokine in PE, modulates the immune response by increasing vascular permeability, lymphocyte activation, and IL-6 and IL-8 production upon hypoxic placental stress (54-56). In PE, it is known that bioactive factors like angiotensin II Type $1 \mathrm{Re}-$ ceptor Agonistic Autoantibodies ( $\mathrm{AT}_{1}$ - AA) stimulates NADPH oxidase through the AT1 receptor and triggers trophoblast and vascular smooth muscle cells to produce ROS, activate nuclear factor kappa B (NF-kB) and elicit inflammation (57). In line with our findings in chapter 5, Khalil et al. already suggested $\mathrm{AT}_{1}$-AA released by the placenta could cause severe vasoconstriction. Previously it has been proposed that besides activating the AT- 1 receptor, increased levels of placental $\mathrm{AT}_{1}$-AA may stimulate endothelial cells to produce ET-1, which may further trigger the genesis of preeclamptic hypertension (58). $\mathrm{AT}_{1}$-AA has shown to result in increased circulating sFlt-1 and sEng levels in pregnant rats, which are also known to stimulate the release of ET-1 in the maternal circulation (51).

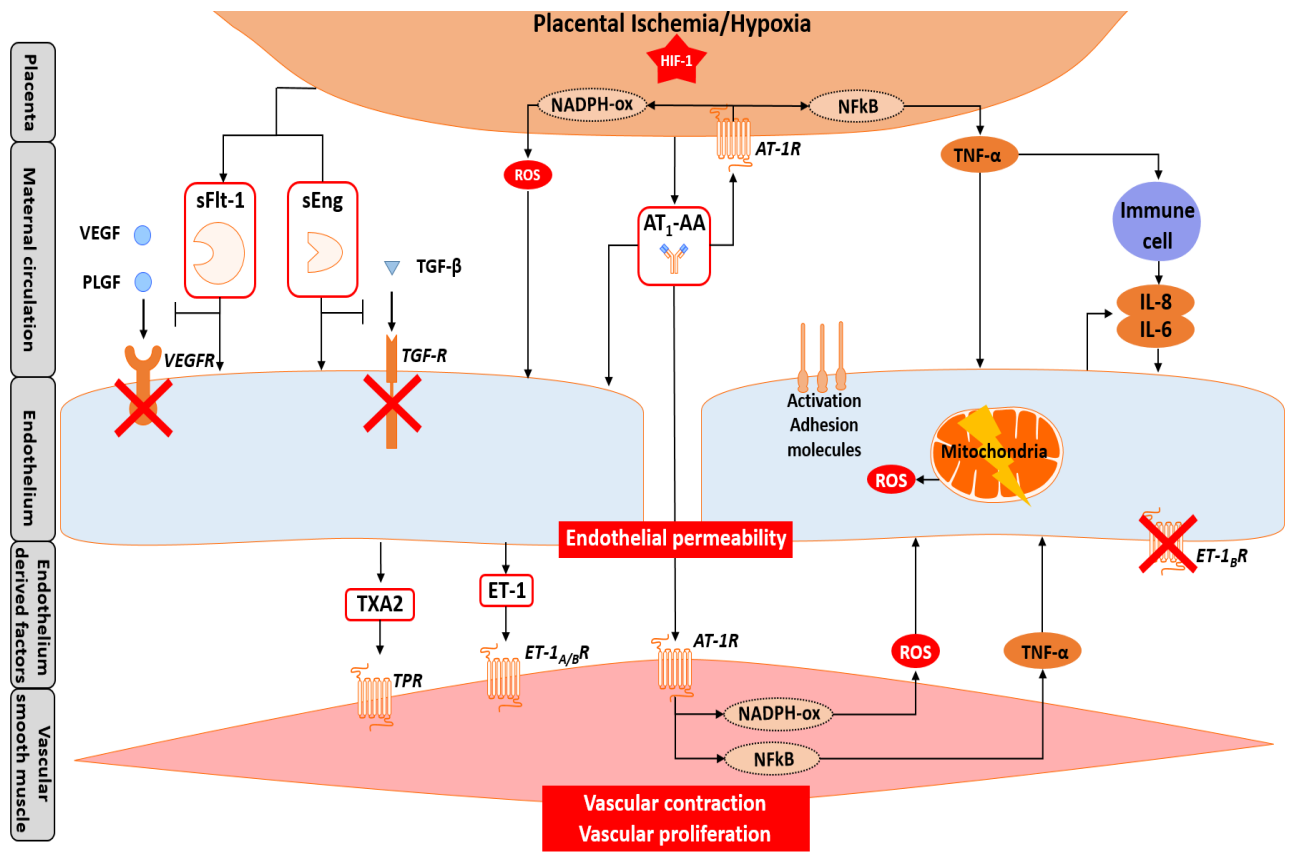

Figure 3. Schematic representation of the proposed sequence of vascular events upon placental hypoxia increasing endothelial permeability and hypertension and vascular proliferation based on the findings of this thesis. Placental ischemia/ hypoxia triggers an increase in HIF-1 followed by 
the release of placental factors such as sFlt-1, sEng and AT $-\mathrm{AA}$, which cause the release of ROS via NADPH oxidase activation and TNF- $\alpha$ upon NFkB activation in placental and smooth muscle cells. Excessive sFlt-1 binds VEGF and PLGF and prevents their angiogenic effects mediated by VEGF receptor binding. Excessive sEng binds TGF- $\beta$ and prevents its angiogenic effect mediated by TGF receptor binding. Decreased activation of the VEGF and TGF receptor disturbs normal endothelial function and increases endothelial permeability. Circulating TNF- $\alpha$ triggers leukocyte and endothelial cells to produce pro-inflammatory cytokines and initiate mitochondrial dysfunction resulting in the formation of ROS, together triggering increased endothelial permeability. Furthermore, sFlt-1, sEng and $\mathrm{AT}_{1}-\mathrm{AA}$ may also trigger endothelial production of the vascular contractile compounds TXA2 and ET-1 which then together with $\mathrm{AT}_{1}$-AA bind their receptor on smooth muscle cells and induce vascular contraction and proliferation. HIF-1, Hypoxia-inducible factor 1; sFlt-1, Soluble fms-like tyrosine kinase-1; sEng, Soluble endoglin; AT -AA, Angiotensin II Type 1 Receptor Agonistic Autoantibody; ROS, Reactive oxygen species; TNF- $\alpha$, Tumor necrosis factor alpha; NF-kB, Nuclear factor kappa B; VEGF, Vascular endothelial growth factor; PLGF, Placental like growth factor; TGF- $\beta$, Transforming growth factor beta 1 .

Besides the direct effects on contractility of the vessels during pregnancy, resulting in maternal hypertension, in chapter 6, we showed that placental HCM also irreversibly modified the vascular system, which is in line with the increased risk for cardiovascular diseases in later life of women who suffered from PE. By comparing cross-sectional areas of the lumen, media, and adventitia of arteries exposed to placental HCM, the cross-sectional area of the media, containing the smooth muscle cells, was increased compared to the control. To examine the cause for the increased cross-sectional area of the media, vascular proliferation was assessed in the same arteries, which was already significantly increased after 1 day exposure to placental HCM. Both the increased cross-sectional area and vascular proliferation were concentration-dependently avoided by the presence of dietary quercetin or RRR- $\alpha$-tocopherol during the placental exposure to hypoxia. To confirm if the protective effect of quercetin is due to its antioxidant properties, RRR- $\alpha$-tocopherol was tested as well in our study. RRR- $\alpha$-tocopherol is a naturally occurring and most active form and which have already been used as a fertility enhancement and to improve uterine artery blood flow (59-61). This indicated as well that oxidative stress is the main driver of the altered placental secretome upon hypoxia, which causes the vascular abnormalities observed in our study.

As illustrated in figure 3, $\mathrm{AT}_{1}$-AA in PE or upon placental hypoxia have not only proved to have a profound contribution to the increased vasoconstriction in $\mathrm{PE}$, but is also known to promote smooth muscle growth upon AT1 receptor activation (41, 62). Together with pro-inflammatory cytokines, $\mathrm{AT}_{1}$-AA promotes the endothelial release of vasoactive factors such as ET-1 and TXA2, which stimulates smooth muscle proliferation furthermore through the ETB and thromboxane receptor respectively $(41,63,64)$. Placental HCM caused a sustained increase in vascular contractile responsiveness to U46619, which is in line with a similar increase in vascular con- 
tractility to TXA2, angiotensin II (AngII) and ET-1 of omental arteries observed in women suffering from PE (65).

\section{The link of PE with future cardiovascular risks and pitfalls of epidemiological studies}

Epidemiological evidence showed a clear link between previous PE and future cardiovascular risk (16). Besides predisposing women to PE, also hypertension, microalbuminuria, dyslipidemia, a pro-inflammatory phenotype, obesity, and insulin resistance are observed in higher numbers in women in later life after a PE-complicated delivery (66). It cannot be ruled out that maternal adaptations to pregnancy lead to long-term metabolic, cardiovascular and inflammatory changes, and that PE leaves a permanent 'scar' (67). To better investigate whether these observations in later life are indeed caused by PE, prospective studies with pre-pregnancy risk factor assessment are needed. However, because of the overall incidence of early PE, which is estimated to be less than 1 in 500 pregnancies, the inclusion of at least 100,000 or more pregnancies should be included in the study to gain sufficient power (67). At present, such studies are hardly feasible and are not likely to be attempted in the near future. This limitation further underlines the need for a molecular mechanistic approach as used in this thesis, to study identify how a PE-complicated placenta contributes to the long term consequences related to future metabolic and cardiovascular events in the mother.

\section{Conclusion}

$\mathrm{PE}$ is a hypertensive disorder of pregnancy that continues to affect 10 million pregnant women and kills 76,000 mothers and 500,000 babies per year worldwide (68). The lack of understanding of the molecular mechanisms involved in the early causes of PE and its unknown clinical subtypes are the main reasons for the delay in diagnosis and access to appropriate care and the lack of curative options besides preterm delivery of the fetus. Better understanding of the molecular mechanisms of PE may serve as a tool in the identification of PE subtypes and developing prediction strategies, early prevention options, and new directions for a possible cure for this devastating disease. The outcomes of this thesis illustrate compensatory histological villous maturation in PE complicated placentae, which provide pathologists with a tool to histologically underpin the clinical diagnosis of PE. Besides the morphological consequences of PE, we comprehensively demonstrate increased oxidative stress, which mainly originates from mitochondria, decreased mitochondrial content and signs for reduced mitochondrial biogenesis, and increased mitophagy and mitochondrial fission in placentae complicated with PE or after exposure to hypoxia. Our 
findings imply that mitochondrially targeted antioxidant-based intervention aimed at preventing mitochondrial dysfunction and excessive ROS formation may have therapeutic potential in pregnancy complications like PE. Being able to adequately identify and understand these distinct alterations in the placenta within the clinical setting is a critical first step in the generation of reliable predictive and diagnostic biomarkers, new therapeutic approaches, better clinical management, and identification of PE subtypes. Furthermore, we found that placental oxidative stress upon placental hypoxia triggers the placental secretome resulting in vascular contraction mediated via the AT1 and ET-1 receptors, and increases endothelial permeability. Increased vascular responsiveness to contractile compounds like TXA2 was associated with increased vascular proliferation and subsequent increased arterial thickness of the media. A better understanding of the complex interplay between the stressed placenta in PE and the maternal cardiovascular system may help in the identification of new serum biomarkers for the prediction of $\mathrm{PE}$ and in the design of new diagnostic approaches for better clinical management. It will also improve our knowledge of the long-lasting maternal vascular consequences opening new postpartum treatment and follow-up strategies. 


\section{References}

1. Fox A, McHugh S, Browne J, Kenny LC, Fitzgerald A, Khashan AS, et al. Estimating the Cost of Preeclampsia in the Healthcare System: Cross-Sectional Study Using Data From SCOPE Study (Screening for Pregnancy End Points). Hypertension. 2017;70(6):1243-9.

2. Magee RJ, Santillan MK, Betz AM, DuBose LE, O'Deen A, Holwerda SW, et al. Arterial stiffness but not physical activity levels and vascular endothelial function are altered in early/mid pregnancy in women who develop preeclampsia. The FASEB Journal. 2018;32(1_supplement):715.13-.13.

3. Blois SM, Dechend R, Barrientos G, Staff AC. A potential pathophysiological role for galectins and the renin-angiotensin system in preeclampsia. Cellular and molecular life sciences. 2015;72(1):39-50.

4. Pijnenborg R. Trophoblast invasion. Reproductive Medicine Review. 1994;3(1):53-73.

5. Fazleabas A, Kim J, Strakova Z. Implantation: embryonic signals and the modulation of the uterine environment - a review. Placenta. 2004;25:S26-S31.

6. Kwiatkowski S, Kwiatkowska E, Rzepka R, Torbe A, Dolegowska B. Ischemic placental syndrome--prediction and new disease monitoring. J Matern Fetal Neonatal Med. 2016;29(12):2033-9.

7. Thilaganathan B. Placental syndromes: getting to the heart of the matter. Ultrasound Obstet Gynecol. 2017;49(1):7-9.

8. Granger DN, Kvietys PR. Reperfusion injury and reactive oxygen species: The evolution of a concept. Redox Biol. 2015;6:524-51.

9. Biswas SK. Does the Interdependence between Oxidative Stress and Inflammation Explain the Antioxidant Paradox? Oxid Med Cell Longev. 2016;2016:5698931. 10. Krock BL, Skuli N, Simon MC. Hypoxia-induced angiogenesis: good and evil. Genes Cancer. 2011;2(12):1117-33.

11. Tal R. The role of hypoxia and hypoxia-inducible factor-1alpha in preeclampsia pathogenesis. Biol Reprod. 2012;87(6):134.

12. Covarrubias AE, Lecarpentier E, Lo A, Salahuddin S, Gray KJ, Karumanchi SA, et al. AP39, a Modulator of Mitochondrial Bioenergetics, Reduces Antiangiogenic Response and Oxidative Stress in Hypoxia-Exposed Trophoblasts: Relevance for Preeclampsia Pathogenesis. Am J Pathol. 2019;189(1):104-14.

13. Charnock-Jones DS, Kaufmann P, Mayhew TM. Aspects of human fetoplacental vasculogenesis and angiogenesis. I. Molecular regulation. Placenta. 2004;25(2-3):103-13.

14. Sibai BM. Maternal and uteroplacental hemodynamics for the classification and prediction of preeclampsia. Am Heart Assoc; 2008. 
15. Gathiram P, Moodley J. Pre-eclampsia: its pathogenesis and pathophysiolgy. Cardiovasc J Afr. 2016;27(2):71-8.

16. Khodzhaeva ZS, Kogan YA, Shmakov RG, Klimenchenko NI, Akatyeva AS, Vavina OV, et al. Clinical and pathogenetic features of early- and late-onset pre-eclampsia. J Matern Fetal Neonatal Med. 2016;29(18):2980-6.

17. Madazli R, Yuksel MA, Imamoglu M, Tuten A, Oncul M, Aydin B, et al. Comparison of clinical and perinatal outcomes in early-and late-onset preeclampsia. Archives of gynecology and Obstetrics. 2014;290(1):53-7.

18. Raymond D, Peterson E. A critical review of early-onset and late-onset preeclampsia. Obstetrical \& gynecological survey. 2011;66(8):497-506.

19. Li X, Guo P, Xue Y, Gou W, Tong M, Chen Q. An analysis of the differences between early and late preeclampsia with severe hypertension. Pregnancy Hypertension: An International Journal of Women's Cardiovascular Health. 2016;6(1):47-52. 20. Vatten LJ, Skjærven R. Is pre-eclampsia more than one disease? BJOG: An International Journal of Obstetrics \& Gynaecology. 2004;111(4):298-302.

21. Benton SJ, Leavey K, Grynspan D, Cox BJ, Bainbridge SA. The clinical heterogeneity of preeclampsia is related to both placental gene expression and placental histopathology. American journal of obstetrics and gynecology. 2018;219(6):604. e1-. e25.

22. Salles AM, Galvao TF, Silva MT, Motta LC, Pereira MG. Antioxidants for preventing preeclampsia: a systematic review. ScientificWorldJournal. 2012;2012:243476.

23. Aouache R, Biquard L, Vaiman D, Miralles F. Oxidative Stress in Preeclampsia and Placental Diseases. Int J Mol Sci. 2018;19(5).

24. Tong W, Giussani DA. Preeclampsia link to gestational hypoxia. J Dev Orig Health Dis. 2019:1-12.

25. Chiarello DI, Abad C, Rojas D, Toledo F, Vazquez CM, Mate A, et al. Oxidative stress: Normal pregnancy versus preeclampsia. Biochim Biophys Acta Mol Basis Dis. 2018.

26. Murphy MP. How mitochondria produce reactive oxygen species. Biochemical journal. 2009;417(1):1-13.

27. Aljunaidy MM, Morton JS, Kirschenman R, Phillips T, Case CP, Cooke C-LM, et al. Maternal treatment with a placental-targeted antioxidant (MitoQ) impacts offspring cardiovascular function in a rat model of prenatal hypoxia. Pharmacological research. 2018;134:332-42.

28. Nuzzo AM, Camm EJ, Sferruzzi-Perri AN, Ashmore TJ, Yung HW, Cindrova-Davies T, et al. Placental Adaptation to Early-Onset Hypoxic Pregnancy and Mitochondria-Targeted Antioxidant Therapy in a Rodent Model. Am J Pathol. 2018;188(12):2704-16. 
29. Aljunaidy MM, Morton JS, Cooke CM, Davidge ST. Prenatal hypoxia and placental oxidative stress: linkages to developmental origins of cardiovascular disease. Am J Physiol Regul Integr Comp Physiol. 2017;313(4):R395-R9.

30. Burton GJ, Yung HW, Murray AJ. Mitochondrial - Endoplasmic reticulum interactions in the trophoblast: Stress and senescence. Placenta. 2017;52:146-55.

31. Larsen S, Nielsen J, Hansen CN, Nielsen LB, Wibrand F, Stride N, et al. Biomarkers of mitochondrial content in skeletal muscle of healthy young human subjects. J Physiol. 2012;590(14):3349-60.

32. Xu Z, Jin X, Cai W, Zhou M, Shao P, Yang Z, et al. Proteomics Analysis Reveals Abnormal Electron Transport and Excessive Oxidative Stress Cause Mitochondrial Dysfunction in Placental Tissues of Early-Onset Preeclampsia. Proteomics Clin Appl. 2018;12(5):e1700165.

33. Zhou X, Han T-L, Chen H, Baker PN, Qi H, Zhang H. Impaired mitochondrial fusion, autophagy, biogenesis and dysregulated lipid metabolism is associated with preeclampsia. Experimental cell research. 2017;359(1):195-204.

34. Yu J, Guo X, Chen R, Feng L. Downregulation of mitofusin 2 in placenta is related to preeclampsia. BioMed Research International. 2016;2016.

35. Padmini E, Lavanya S, Uthra V. Preeclamptic placental stress and over expression of mitochondrial HSP70. Clinical chemistry and laboratory medicine. 2009;47(9):1073-80.

36. Postigo L, Heredia G, Illsley NP, Torricos T, Dolan C, Echalar L, et al. Where the $\mathrm{O} 2$ goes to: preservation of human fetal oxygen delivery and consumption at high altitude. The Journal of physiology. 2009;587(3):693-708.

37. Lopez-Armada MJ, Riveiro-Naveira RR, Vaamonde-Garcia C, Valcarcel-Ares MN. Mitochondrial dysfunction and the inflammatory response. Mitochondrion. 2013;13(2):106-18.

38. Ausman J, Abbade J, Ermini L, Farrell A, Tagliaferro A, Post M, et al. Ceramide-induced BOK promotes mitochondrial fission in preeclampsia. Cell Death Dis. 2018;9(3):298.

39. McCarthy CM, Kenny LC. Mitochondrial [dys]function; culprit in pre-eclampsia? Clin Sci (Lond). 2016;130(14):1179-84.

40. Williams JA, Zhao K, Jin S, Ding W-X. New methods for monitoring mitochondrial biogenesis and mitophagy in vitro and in vivo. Experimental Biology and Medicine. 2017;242(8):781-7.

41. Yu W, Gao W, Rong D, Wu Z, Khalil RA. Molecular determinants of microvascular dysfunction in hypertensive pregnancy and preeclampsia. Microcirculation. 2018:e12508.

42. Sánchez-Aranguren LC, Prada CE, Riaño-Medina CE, Lopez M. Endothelial dysfunction and preeclampsia: role of oxidative stress. Frontiers in physiology. 
2014;5:372.

43. Vangrieken P, Vanterpool SF, van Schooten FJ, Al-Nasiry S, Andriessen P, Degreef $\mathrm{E}$, et al. Histological villous maturation in placentas of complicated pregnancies. Histol Histopathol. 2020:18205.

44. Crews JK, Herrington JN, Granger JP, Khalil RA. Decreased endothelium-dependent vascular relaxation during reduction of uterine perfusion pressure in pregnant rat. Hypertension. 2000;35(1 Pt 2):367-72.

45. Gilbert JS, Babcock SA, Granger JP. Hypertension produced by reduced uterine perfusion in pregnant rats is associated with increased soluble fms-like tyrosine kinase-1 expression. Hypertension. 2007;50(6):1142-7.

46. Fushima T, Sekimoto A, Minato T, Ito T, Oe Y, Kisu K, et al. Reduced Uterine Perfusion Pressure (RUPP) Model of Preeclampsia in Mice. PLoS One. 2016;11(5):e0155426.

47. Vangrieken P, Al-Nasiry S, Janssen GMJ, Weseler AR, Spaanderman ME, Bast A, et al. The direct and sustained consequences of severe placental hypoxia on vascular contractility. PLoS One. 2018;13(8):e0202648.

48. Boots AW, Haenen GR, Bast A. Health effects of quercetin: from antioxidant to nutraceutical. European journal of pharmacology. 2008;585(2-3):325-37.

49. Matsubara K, Higaki T, Matsubara Y, Nawa A. Nitric oxide and reactive oxygen species in the pathogenesis of preeclampsia. Int J Mol Sci. 2015;16(3):460014.

50. Sava RI, March KL, Pepine CJ. Hypertension in pregnancy: Taking cues from pathophysiology for clinical practice. Clin Cardiol. 2018;41(2):220-7.

51. Palei AC, Spradley FT, Warrington JP, George EM, Granger JP. Pathophysiology of hypertension in pre-eclampsia: a lesson in integrative physiology. Acta Physiol (Oxf). 2013;208(3):224-33.

52. Powe CE, Levine RJ, Karumanchi SA. Preeclampsia, a disease of the maternal endothelium: the role of antiangiogenic factors and implications for later cardiovascular disease. Circulation. 2011;123(24):2856-69.

53. Wang Y, Lewis DF, Gu Y, Zhang Y, Alexander JS, Granger DN. Placental trophoblast-derived factors diminish endothelial barrier function. J Clin Endocrinol Metab. 2004;89(5):2421-8.

54. LaMarca B, Speed J, Fournier L, Babcock SA, Berry H, Cockrell K, et al. Hypertension in response to chronic reductions in uterine perfusion in pregnant rats: effect of tumor necrosis factor-alpha blockade. Hypertension. 2008;52(6):1161-7.

55. Matthiesen L, Berg G, Ernerudh J, Ekerfelt C, Jonsson Y, Sharma S. Immunology of preeclampsia. Chem Immunol Allergy. 2005;89:49-61.

56. Possomato-Vieira JS, Khalil RA. Mechanisms of Endothelial Dysfunction in Hypertensive Pregnancy and Preeclampsia. Adv Pharmacol. 2016;77:361-431. 
57. Dechend R, Viedt C, Muller DN, Ugele B, Brandes RP, Wallukat G, et al. AT1 receptor agonistic antibodies from preeclamptic patients stimulate NADPH oxidase. Circulation. 2003;107(12):1632-9.

58. George EM, Granger JP. Endothelin: key mediator of hypertension in preeclampsia. Am J Hypertens. 2011;24(9):964-9.

59. Rigotti A. Absorption, transport, and tissue delivery of vitamin E. Molecular aspects of medicine. 2007;28(5-6):423-36.

60. Şimşek M, Naziroğlu M, Şimşek H, Cay M, Aksakal M, Kumru S. Blood plasma levels of lipoperoxides, glutathione peroxidase, beta carotene, vitamin A and E in women with habitual abortion. Cell Biochemistry and Function: Cellular biochemistry and its modulation by active agents or disease. 1998;16(4):227-31 .

61. Mesdaghinia E, Mohammad-Ebrahimi B, Foroozanfard F, Banafshe HR. The effect of vitamin $\mathrm{E}$ and aspirin on the uterine artery blood flow in women with recurrent abortion: A single-blind randomized controlled trial. International Journal of Reproductive BioMedicine. 2017;15(10):635.

62. Jia G, Aroor AR, Meininger GA, Sowers JR. Glucose, Insulin and Potential Strategies of Vascular Stiffening. Blood Pressure and Arterial Wall Mechanics in Cardiovascular Diseases: Springer; 2014. p. 423-34.

63. Majed BH, Khalil RA. Molecular mechanisms regulating the vascular prostacyclin pathways and their adaptation during pregnancy and in the newborn. Pharmacological reviews. 2012;64(3):540-82.

64. Dong F, Zhang X, Wold LE, Ren Q, Zhang Z, Ren J. Endothelin-1 enhances oxidative stress, cell proliferation and reduces apoptosis in human umbilical vein endothelial cells: role of ETB receptor, NADPH oxidase and caveolin-1. Br J Pharmacol. 2005;145(3):323-33.

65. Belfort MA, Saade GR, Suresh M, Kramer W, Vedernikov YP. Effects of selected vasoconstrictor agonists on isolated omental artery from premenopausal nonpregnant women and from normal and preeclamptic pregnant women. Am J Obstet Gynecol. 1996;174(2):687-93.

66. Murphy MS, Tayade C, Smith GN. Evidence of inflammation and predisposition toward metabolic syndrome after pre-eclampsia. Pregnancy Hypertension: An International Journal of Women's Cardiovascular Health. 2015;5(4):354-8.

67. van Rijn BB. Early-onset preeclampsia: Constitutional factors and consequences for future pregnancy outcome and cardiovascular health: Utrecht University; 2008.

68. Giachini FR, Galaviz-Hernandez C, Damiano AE, Viana M, Cadavid A, Asturizaga $\mathrm{P}$, et al. Vascular dysfunction in mother and offspring during preeclampsia: contributions from latin-American countries. Current hypertension reports. 2017;19(10):83. 



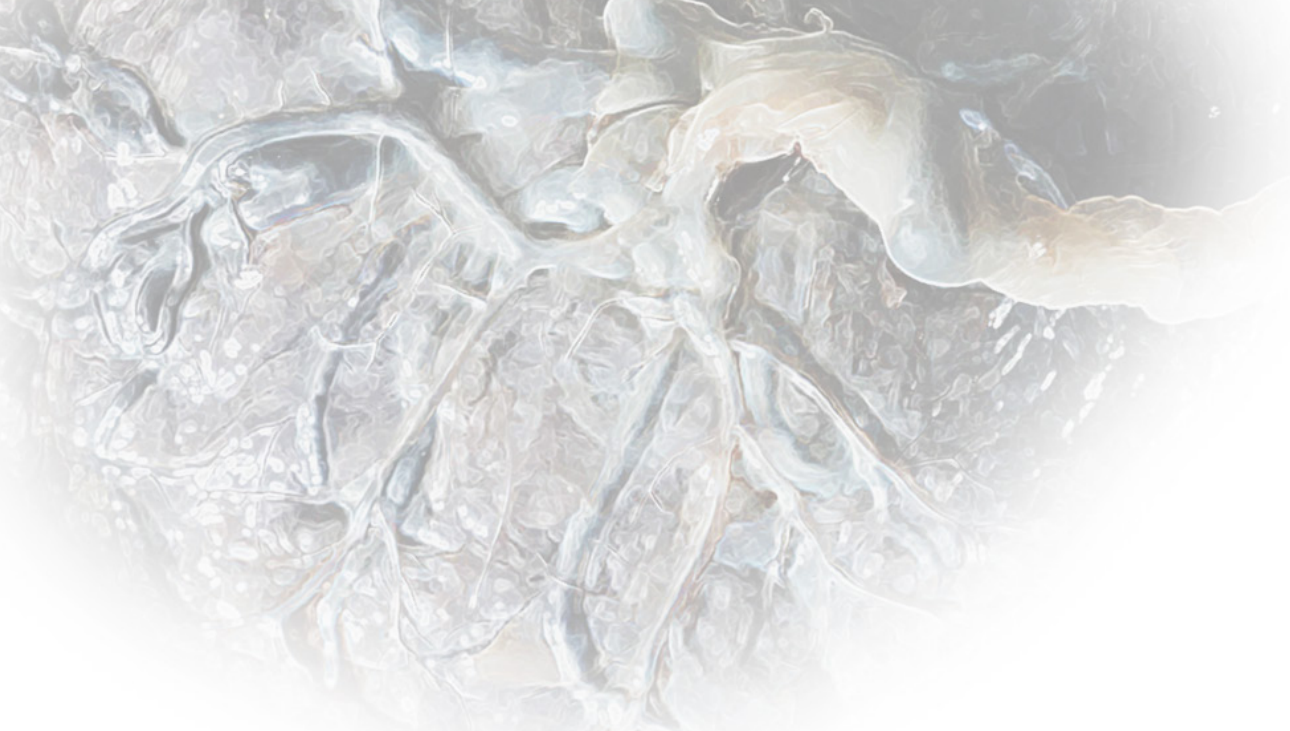




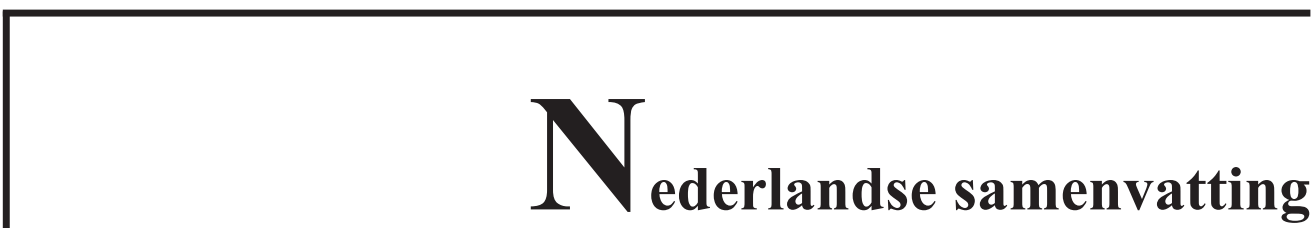


Dit proefschrift richt zich op de pathofysiologie van preeclampsie (PE), beter bekend als zwangerschapsvergiftiging. Het biedt een reeks van geïdentificeerde parameters aan die betrekking hebben op versnelde placentale ontwikkeling, die vervolgens gebruikt kunnen worden om meer subtypes van PE te identificeren. Het onderscheiden van meer subtypes van PE zal vervolgens bijdrage aan een meer gerichte klinische behandeling en bij het voorkomen van herhaalde PE in een volgende zwangerschap (Hoofdstuk 2). Verder biedt dit proefschrift nieuwe inzichten in de bijdrage van mitochondriën in het mediëren van placentale oxidatieve stress in PE-gecompliceerde placenta's of na blootstelling aan hypoxie (verminderde zuurstof spanning) (Hoofdstuk 3 en 4). Bovendien creëert dit proefschrift een beter begrip in het samenspel tussen een placenta die blootgesteld is aan hypoxie en het vaatstelsel in termen van acute en aanhoudende vaatcontractiliteit, morfologie en endotheliale integriteit (Hoofdstuk 5 en 6).

Bij PE wordt aangenomen dat een versnelde rijping van placentale villi (die het contact oppervlakte vormen tussen het maternale bloed en de foetale circulatie) optreedt als compensatie voor een afwijkende bloedtoevoer naar de placenta. Echter is er nog steeds weinig bekend over deze specifieke morfologische aanpassingen van de placenta. Een gedetailleerde profilering van PE placenta's zal ons helpen in het typeren van PE-suptypes en in het beter herkennen van deze structurele patronen, wat zal bijdragen aan het vroegtijdig opsporen van deze ziekte tijdens te zwangerschap. Daarom is in hoofdstuk 2 een reeks van morfologische parameters geïdentificeerd om histologische villus maturiteit te evalueren in placenta's gecompliceerd met PE. PE-gecompliceerde placenta's zijn vergeleken met controle placenta's die overeenkomen met hun zwangerschapsduur als ook met volgroeide placenta's. Histologische villus rijping is gekarakteriseerd door middel van 17 histologische markers. Veertien van deze makers geven informatie over de potentiele capaciteit van de placenta om gassen, voedingsstoffen en afvalstoffen uit te wisselen tussen moeder en de foetus en 11 van deze markers tonen versnelde histologische villus rijping aan in PE placenta's in vergelijking met de controle groep. Data in dit proefschrift laat zien dat PE placenta's sneller overgaan van groei naar rijping van de placentale villi in vergelijking met controle placenta's.

Verder is het algemeen aangenomen dat oxidatieve stress een belangrijke rol speelt in de pathofysiologie van PE. Echter is het ontstaan van placentale oxidatieve stress in PE nog steeds onduidelijk. In dit proefschrift (Hoofdstuk 3 en 4), is aangetoond dat PE-gecompliceerde placenta's en placenta's of BeWo trofoblast cellen (cellen die de eerste barrière vormen tussen het maternale en foetale circulatie) die blootgesteld zijn aan hypoxie, gekenmerkt worden door hogere niveaus van geoxideerd 
glutathion (GSSG), een hoger antioxidant vermogen en hogere mRNA-niveaus van het mitochondrieel gelokaliseerde antioxidant enzym mangaan-afhankelijk superode dismutase 2 (MnSOD2) in vergelijking met de controle groep. De toegenomen oxidatieve stress, verhoogde mitochondrieel-specifieke antioxidant expressie in PE, en het feit dat mitochondriën de belangrijkste intracellulaire plaats zijn voor cellulaire $\mathrm{O}_{2}$-consumptie, suggereert dat mitochondriën een belangrijke bron vormen voor reactieve zuurstofradicalen in PE-placenta's of na blootstelling aan hypoxie. In overeenstemming met deze waarneming, zorgt het mitochondrieel-specifieke antioxidant MitoQ voor een concentratie-afhankelijke verlaging van de intracellulaire productie van reactieve zuurstofradicalen en een verhoudingsgewijsde verlaging van GSSG niveaus in BeWo tophoblasten tijdens de blootstelling aan hypoxie (Hoofdstuk 4). Bovendien zijn in hoofdstuk 3 en 4 aanwijzingen gevonden voor een significante verlagingen van het aantal mitochondriën in PE-placenta's en BeWo-trofoblasten die blootgesteld zijn aan hypoxie in vergelijking met de controles. Onze gegevens geven aan dat in reactie op PE of hypoxie het energiemetabolisme van de placenta verschuift van oxidatieve fosforylering naar glycolyse. De verhoogde afhankelijkheid van de glycolyse in PE-placenta's kan een gevolg zijn van lagere $\mathrm{O}_{2}$-spiegels, een verminderde aantal mitochondriën en een verminderde werking van hun elektronen transport keten. Deze verschuiving naar anaerobe ademhaling (opwekken van energie zonder het gebruik van $\mathrm{O}_{2}$ ) kan een beschermende reactie van de placenta zijn voor de verminderde placentale perfusie bij $\mathrm{PE}$ om zo $\mathrm{O}_{2}$ te sparen voor de groeiende foetus. Bovendien veroorzaken disfunctionele mitochondriën niet alleen een overmatige productie van reactieve zuurstofradicalen, maar veroorzaken ze ook een ontstekingsreactie en apoptose (geprogrammeerde celdood), die beide gekend zijn om betrokken te zijn bij de pathogenese van PE. Een verhoogde productie van mitochondriale reactieve zuurstofradicalen in de placenta, kan mitochondriaal DNA direct beschadigen en zo de aanmaak van mitochondriën (biogenese) remmen en de afbraak (mitofagie) stimuleren. In overeenstemming met de verlaagde hoeveelheid mitochondriën, zijn de mRNA- en eiwitniveaus van de belangrijkste moleculen die betrokken zijn bij de regulering van de mitochondriale biogenese lager in PE-placenta's en placenta's of BeWo-trofoblasten die blootgesteld zijn aan hypoxie, terwijl die van de mitofagie verhoogd zijn in vergeleken met de controles.

De pathofysiologische processen die ten grondslag liggen aan de vroege aanvang van $\mathrm{PE}$ omvatten een afwijkende ontwikkeling van de placenta en apoptose van invasieve trofoblast cellen wat leidt tot onvoldoende aanpassing van spirale arteriën wat resulteert in onvoldoende bloedtoevoer naar de placenta. De daaropvolgende verlaagde en schommelende toevoer van $\mathrm{O}_{2}$ naar de placenta resulteert in oxidatieve stress en de productie van een verscheidenheid aan door de placenta uitgescheiden 
factoren (secretoom) die gezamenlijk diepgaande effecten hebben op de bloeddrukregulatie van de moeder. In samenspraak met voorgaande studies tonen gegevens in dit proefschrift (Hoofdstuk 5) aan dat placenta-hypoxisch geconditioneerd medium (HCM) de vasoconstrictie via de Angiotensine II-receptor type 1 (AT1) en (ET-1) -receptor verhoogt en de vasculaire contractiliteit van een tromboxaan-A2 (TXA2) agonist analoog (U46619) verhoogd. Bij een gecombineerde blootstelling van de placenta aan hypoxie en het antioxidant quercetine, kunnen de acute en aanhoudende vasculaire effecten op het placenta-geconditioneerde medium op een concentratieafhankelijke manier worden voorkomen. Quercetine is geselecteerd voor deze studie, omdat het de meest voorkomende flavonoïde is die in onze voeding voorkomt. Om te testen of deze effecten endotheel-gemedieerd zijn, zijn dezelfde condities ook intraluminaal getest (Hoofdstuk 6). Intraluminale toediening van hetzelfde HCM induceert ook vasculaire contractie, wat suggereert dat de contractiele respons door het HCM mogelijk endotheel-afhankelijk is, wat ons zal helpen bij de selectie van nieuwe therapeutische strategieën.

Ter ondersteuning van deze gegevens tonen onze gegevens in hoofdstuk 6 aan dat de vasculaire permeabiliteit voor kaliumchloride $(\mathrm{KCl})$ toeneemt na intraluminale blootstelling aan HCM van de placenta in vergelijking met de controle. Bovendien verminderd HCM van zowel de placenta als BeWo-cellen de levensvatbaarheid van endotheelcellen en verhoogd het de IL-6- en IL-8-cytokine-expressie en de productie van reactieve zuurstofradicalen in endotheelcellen. Tumornecrosefactor alfa $(\mathrm{TNF}-\alpha)$, een bekend verhoogd pro-inflammatoire cytokine in PE, moduleert het immuunrespons door de vasculaire permeabiliteit, lymfocytenactivatie en IL-6- en IL-8-productie te verhogen bij hypoxische stress in de placenta. Bij PE is het bekend dat bioactieve factoren zoals angiotensine II Type 1 Receptor Agonistic Autoantibodies ( $\mathrm{AT}_{1}$-AA), NADPH-oxidase stimuleert via de AT1-receptor en zo trofoblast cellen en vasculaire gladde spiercellen activeert om reactieve zuurstofradicalen te produceren, nucleaire factor kappa $\mathrm{B}(\mathrm{NF}-\mathrm{kB})$ te activeren en vervolgens een ontstekingsreactie te initiëren. In lijn met onze bevindingen in hoofdstuk 5, suggereerde Khalil et al. dat $\mathrm{AT}_{1}$-AA, vrijgegeven door de placenta, ernstige vaatvernauwing kan veroorzaken. Eerder is voorgesteld dat, naast het activeren van de AT-1-receptor, verhoogde niveaus van $\mathrm{AT}_{1}$ - $\mathrm{AA}$ van de placenta, endotheelcellen kan activeren om ET-1 te produceren, wat het ontstaan van PE hypertensie verder kan stimuleren.

Naast de directe effecten op de contractiliteit van bloedvaten tijdens de zwangerschap, resulterend in maternale hypertensie, laten we in hoofdstuk 6 zien dat HCM van de placenta ook het vaatstelsel onomkeerbaar veranderd, wat in lijn is met het verhoogd risico op hart- en vaatziekten op latere leeftijd bij vrouwen na PE. Door 
dwarsdoorsnedeoppervlakken van het lumen, de media en de adventitia van arteriën die aan HCM van de placenta blootgesteld zijn te vergelijken, is de dwarsdoorsnedeoppervlakte van de media, die de gladde spiercellen bevat, vergroot in vergelijking met die van de controle. Om de oorzaak van het vergrote dwarsdoorsnedeoppervlak van de media te onderzoeken, is de vasculaire celdeling beoordeeld in dezelfde arteriën, die significant toeneemt na 1 dag blootstelling aan het HCM van de placenta. Zowel de toename in dwarsdoorsnedeoppervlak als de vasculaire proliferatie (celdeling) kunnen concentratieafhankelijk vermeden worden door de aanwezigheid van quercetine of RRR- $\alpha$-tocoferol tijdens de placentale blootstelling aan hypoxie. Om te bevestigen of het beschermend effect van quercetine te danken is aan de antioxiderende eigenschappen, is RRR- $\alpha$-tocoferol ook getest in ons onderzoek. RRR$\alpha$-tocoferol is een natuurlijk voorkomende en de meest actieve vorm en is al eerder gebruikt als vruchtbaarheidsverbetering ter verbetering van de doorbloeding van de baarmoederader. Deze bevindingen bevestigen opnieuw dat oxidatieve stress de belangrijkste motor is van het gewijzigde placentaire secretoom na blootstelling aan hypoxie, wat de afwijkingen in functie en morfologie van de vaten veroorzaakt.

Concluderend, PE is een hypertensieve zwangerschapscomplicatie die nog steeds 10 miljoen zwangere vrouwen treft en wereldwijd resulteert in de sterfte van 76.000 moeders en 500.000 baby's. Het gebrek aan inzicht in de moleculaire mechanismen die betrokken zijn bij de vroege oorzaken van PE en de mogelijk ontbrekende klinische subtypes ervan, zijn de belangrijkste redenen voor de vaak late diagnose en toegang tot passende zorg en het gebrek aan therapeutische opties naast een vroegtijdige bevalling van de foetus. Een beter begrip van de moleculaire mechanismen van PE zal helpen bij het identificeren van PE-subtypes en bij het ontwikkelen van voorspellingsstrategieën, opties voor vroegtijdige preventie en nieuwe mogelijkheden voor een genezing van deze ziekte. De resultaten van dit proefschrift illustreren een compenserende versnelde villus rijping aan in PE-placenta's, die pathologen vervolgens een hulpmiddel kunnen bieden om de klinische diagnose van PE histologische te onderbouwen. Naast de morfologische gevolgen van PE, demonstreren we uitgebreid verhoogde oxidatieve stress, die voornamelijk veroorzaakt is door mitochondriën, een verminderde mitochondriale aanmaak, en verhoogde mitochondriële afbraak in PE-placenta's of na blootstelling aan hypoxie. Onze bevindingen impliceren dat een mitochondrieel gericht, op antioxidanten gebaseerde interventie, met als doel het voorkomen van mitochondriale disfunctie en overmatige vorming van reactieve zuurstofradicalen, therapeutisch potentieel kan hebben bij zwangerschapscomplicaties zoals PE. Het kunnen identificeren en bergrijpen van deze verschillende veranderingen in de placenta binnen een klinische setting, is een cruciale eerste stap in het genereren van betrouwbare voorspellende diagnostische biomark- 
ers, ontwikkelen van meer gerichte therapieën, en het identificeren van PE-subtypen. Verder hebben we ontdekt dat door oxidatieve stress in de placenta na blootstelling aan hypoxie, het placenta secretoom gestimuleerd wordt. Deze veranderde secretie van placentale factoren resulteert in een vasculaire contractie die gemedieerd wordt door de AT1- en ET-1 receptoren en een verhoogde permeabiliteit van het endotheel. Verder leiden deze placentale factoren tot een toegenomen vasculaire contractiliteit voor TXA2 en is dit geassocieerd met een verhoogde vasculaire celdeling gevolgd door een toegenomen verdikking van de arteriële media. Een beter begrip van het complexe samenspel tussen de gestreste placenta in PE en het maternale cardiovasculaire systeem, kan helpen bij de identificatie van nieuwe serum biomarkers voor de voorspelling van PE en bij het ontwerpen van nieuwe diagnostische benaderingen voor een beter klinische behandeling. Het zal ook onze kennis over langdurige vasculaire gevolgen in de moeder verbeteren, waardoor nieuwe postpartum behandelingen en vervolgstrategieën mogelijk worden. 



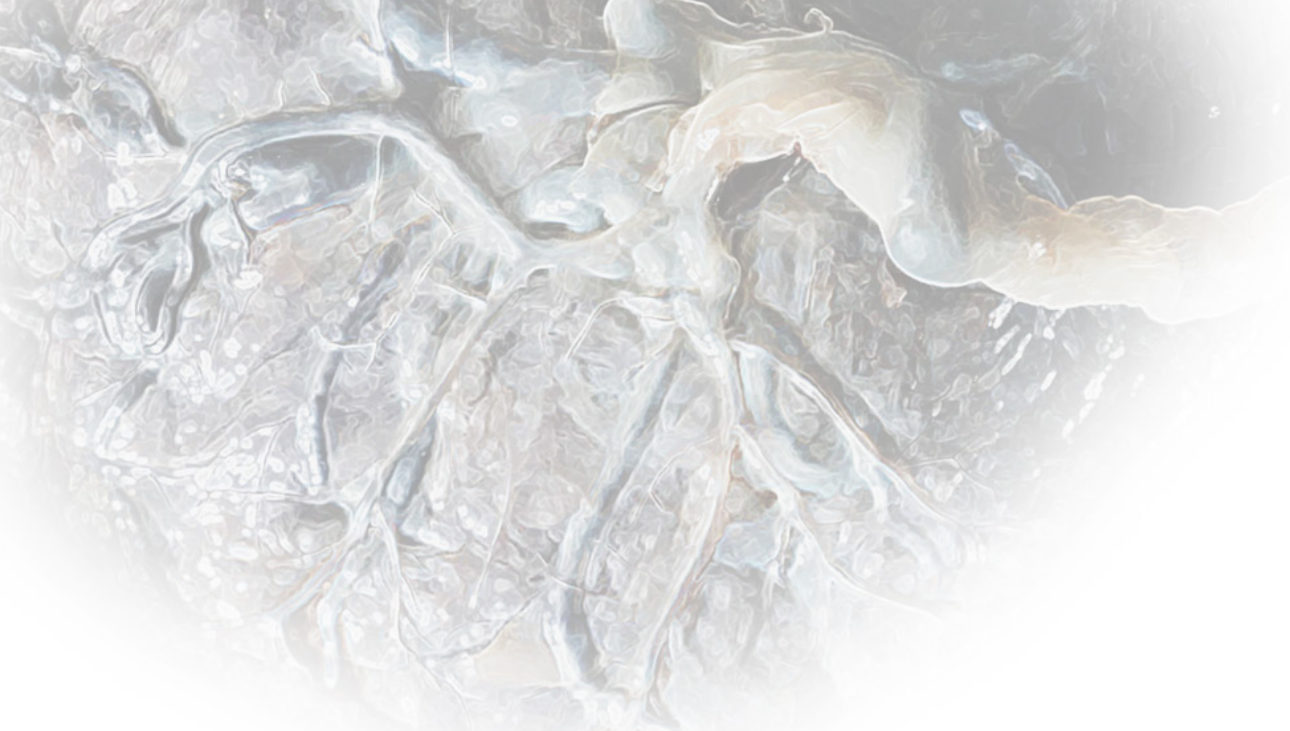




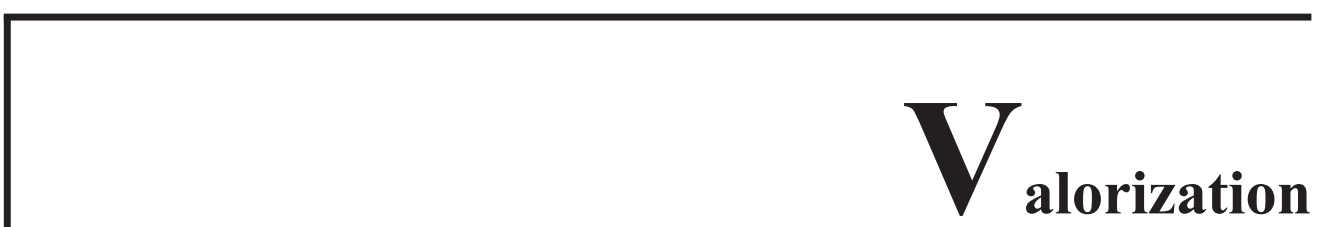


This chapter addresses the relevance and contribution of this thesis to society. The general aim of this project was to investigate the effect of preeclampsia (PE) on placental histological villous maturation and to set up a model for PE to examine the contribution of mitochondria in the generation of placental oxidative stress, its effect on the placental secretome and its subsequent role in acute and sustained vascular contractility, morphology, and endothelial integrity.

Valorization is the term used for the process of value-creation out of knowledge, by making this knowledge suitable and available for economic or societal utilization and to translate this into high-potential products, services, processes, or industrial activity.

First, in this valorization chapter, the current epidemiological data that describes the health status of adults and the economic burden of PE will be presented. Secondly, potential of our results for

- better characterization of the disease subtype to improve diagnosis and subse quent treatment.

- the prevention of PE associated with nutritional interventions via the consump tion of identified diets.

\section{Social and economic impact of $P E$ and relevance of this study}

There are half a million maternal pregnancy-related deaths worldwide of which $1 \%$ occurs in high-income countries (1). PE is an enigmatic and complex hypertensive disorder affecting seemingly healthy pregnant women and carries substantial health risks to both mother and baby. Despite significant research, PE continues to affect 10 million pregnant women and kills 76,000 mothers and 500,000 babies per year worldwide (2). A large regional integrated health care system study in Pennsylvania, US used electronic health records and billing data to identify mother-singleton infant pairs. The final study population included 712 matched mother-infant pairs comparing the medical costs related to uncomplicated pregnancies versus PE-complicated pregnancies. The mean combined maternal and infant medical cost of $\$ 41,790$ were significantly higher than those for the uncomplicated cohort of $\$ 13,187$ and was largely driven by the infant care cost, which was dominated by neonatal intensive care unit costs, which was almost 7 times higher compared to the control group (3). As PE is the leading cause of maternal mortality in the Netherlands, the prevalence of $\mathrm{PE}$ is comparable and high income and middle to low-income countries show similar proportions of maternal deaths related to PE, similar costs are expected in the Nederland's (4). The broad clinical picture of this complication is the reason for a delayed diagnosis, increasing the risk for complications for mother and child and 
it affects maternal health and child's health and wellbeing, especially if the child is born prematurely. PE is a commonly sudden and a distressing life experience, particularly if women felt severely ill, gave birth too early, or if they lost their baby. With secondary neonatal intensive care necessity, PE strongly links to a post-traumatic stress disorder, and depression and women often find difficulties to reintegrate into society after giving birth further demonstrating the broad economic burden of PE.

PE causes not only short but also long term consequences related to future metabolic and cardiovascular events for the mother and child (5). A delay in diagnosis and access to appropriate care is a core cause of PE-related severe morbidity and mortality worldwide and is the main reason for the lack of curative options besides preterm delivery of the fetus. Malformation and subsequent impaired function of the placenta during PE play a pivotal role in the development of suboptimal cardiovascular adaptations during pregnancy leading to a hypertensive-complicated pregnancy. During the disease state, however, there are currently no curative treatment options available, but only symptomatic treatment to correct the underlying cardiovascular shortcomings, which includes optimizing blood-pressure and -flow and protection from possible imminent seizures. Better understanding of the abnormal adaptation processes of the placenta and the maternal vascular system during a PE-complicated pregnancy may result in better diagnostic tools, subsequent shortening of diagnosis, access to appropriate care, and the selection of more targeted therapies. Although several risk factors have been identified, it is difficult to develop effective strategies for the prevention and treatment of PE (6). Strategies applied nowadays are diverse and include antenatal surveillance, modification of lifestyle, dietary interventions, and symptomatic directed pharmacological therapy such as antihypertensive.

Until now, characterization of the two existing subclasses (early and late-onset PE) is only based on the gestational age at diagnosis. Some investigators have considered that the two subtypes of PE represent more than one pathophysiologic process ( 7 , 8 ). This variety of separate pathological entities are yet to be defined and might be an explanation for the failing population-based interventions in women at high risk for developing PE. Allocating all cases to one general PE group may explain why, despite the strong association of PE with oxidative stress, human studies using systematically acting antioxidants like vitamin $\mathrm{C}$ and $\mathrm{E}$ as a treatment for $\mathrm{PE}$ were generally unsuccessful (9). The Barker hypothesis suggests that nutritional exposure in utero has effects that even after birth may last a lifetime. Moreover, that gestational age, which is generally shortened by $\mathrm{PE}$ is predictive of heart disease in the offspring later in life, decades post-partum affecting maternal, foetal and newborn health (3). In addition to these potential long-term effects of $\mathrm{PE}$, maternal diet and nutritional 
status are known to have more immediate effects on maternal, fetal, and newborn health. Over the past 20 years, the importance of preventive nutrition in enhancing pregnancy outcomes has been a very valuable area of research tailoring at a reduction of major birth defects, prolonging pregnancy to term, and avoiding maternal complications. When these complications could be avoided, it not only results in cost savings but also in a reduction in linked emotional and psychological costs. At present, there is still a major opportunity in optimizing pregnancy outcomes of preterm birth-related pregnancy complications like PE by implementing essential micronutrients such as vitamin $\mathrm{C}$ and $\mathrm{E}$ in the diet. Deficiencies in essential micronutrients have profound effects on the physical as well as the maternal capabilities of the offspring and significantly promotes maternal morbidity and mortality. A study by Chappell et al., included 283 pregnant women at risk for $\mathrm{PE}$ in a placebo-controlled trial and daily supplementation of vitamin C (1,000 mg) and vitamin E (400IU) resulted in a $61 \%$ reduction in developing PE. However, other studies using a similar dose of vitamin $\mathrm{C}$ and $\mathrm{E}$ could not replicate the Chappell findings. Inconsistencies in the findings of these and similar studies suggest heterogeneity in population groups, different pre-existing conditions, and other factors independent of the basic research (10-12). The failing identification of PE subgroups based on its clinical presentation and its following limitations in current PE research underlines the need for a better understanding of the molecular mechanisms involved in the development of placental oxidative stress and subsequent alterations in its secretome, which drives the progression of PE. Improved knowledge as presented in this thesis presenting these fundamental mechanisms of PE pathogenesis will provide researchers with a tool to allocate patients to specific PE subgroups, and will help in the formation of homogenous PE test groups and will create better awareness of the diverse pathological image of PE.

As placental maldevelopment and failing adaptation are central to the pathogenesis of PE, in the first research chapter (Chapter 2), we identified 11 parameters of histological villous remodeling, which demonstrate accelerated villous maturation in PE placentae. These identified parameters as presented in our study, not only allow us to study histological adaptations that take place in the placenta complicated with PE, but may also be used to correlate with clinical parameters of the mother and subsequently help in the identification of different clinical subtypes of PE. Advancing our understanding of the pathophysiology underlying PE through the identification of clinically relevant disease subtypes would not only clarify the heterogeneity observed in this disorder but will also help in the development and/or application of etiology-focused screening tools and therapies for PE. These tools would direct the care of women and their offspring affected by PE towards an etiological-based inter- 
vention, which will expand the current symptomatic-focused intervention.

Although mitochondria are forming the major source for ROS and are central to the regulation of cellular metabolism, redox state, and cell fate, few studies have examined the connection between impaired oxygen supply to the placenta and mitochondrial dysfunction and ROS. In our study (Chapter 3 and 4) we now for the first time, comprehensively demonstrate increased oxidative stress, which mainly originates from mitochondria, decreased mitochondrial content, and signs for reduced mitochondrial biogenesis, and increased mitophagy and mitochondrial fission in placentae complicated with PE or after exposure to hypoxia. Our findings imply that mitochondrially targeted antioxidant-based intervention aimed at preventing mitochondrial dysfunction and excessive ROS formation may have therapeutic potential in pregnancy complications like PE.

The specifically identified alterations related to mitochondrial function and oxidative stress may together with the histological adaptations in the placenta found in chapter 2 , be linked to the clinical profile of the mother and serve as a tool to efficiently identify more subgroups of PE in larger cohorts. Moving forward, the use of a PE subtype paradigm may allow for more homogeneous patient population selection in study designs, leading to greater scientific contributions in the understanding of PE pathophysiology, the discovery of highly accurate predictive biomarkers, and targeted therapeutic interventions tailored to each disease subtype.

Placental ischemia/hypoxia leading to oxidative stress (13) results in the production of a variety of placental secreted factors (secretome) that collectively have profound effects on blood flow and arterial pressure regulation. Besides predisposing women to $\mathrm{PE}$, also hypertension, microalbuminuria, dyslipidemia, a pro-inflammatory phenotype, obesity, and insulin resistance are observed in higher numbers in women in later life after a PE-complicated pregnancy (14). In this thesis, we show for the first time that placental oxidative stress upon placental hypoxia triggers the placental secretome resulting in vascular contraction mediated via the AT1 and ET-1 receptors, and increases endothelial permeability. Increased vascular responsiveness to contractile compounds like TXA2 was associated with increased vascular proliferation and subsequent increased arterial thickness of the media. A better understanding of the complex interplay between the stressed placenta in PE and the maternal cardiovascular system may help in the identification of new serum biomarkers for the prediction of $\mathrm{PE}$ and in the design of new diagnostic approaches for better clinical management. It will also improve our knowledge of the long-lasting maternal vascular consequences opening new postpartum treatment and follow-up strategies. 
In conclusion, this thesis provides new insights into the etiology and progression of PE. New diagnostic approaches for better clinical management and early prevention or amelioration of the progression of the disease, will not only positively influence morbidity and mortality rates during pregnancy, but also reduce the risk of maternal and foetal complications in later life. Moreover, in this thesis we improved knowledge on specific histological alterations found in the PE-placenta, comprehensively demonstrated mitochondrial impairments, and following oxidative stress-related alterations in the placental secretome, and its subsequent effect on the vascular system. This knowledge not only provides researchers with a tool that will help in identifying specific PE subgroups but also opens new perspectives in new efficient targeted therapeutic interventions tailored to each disease subtype and well-directed postpartum treatment and follow-up strategies. 


\section{References}

1. Duley L, editor The global impact of pre-eclampsia and eclampsia. Seminars in perinatology; 2009: Elsevier.

2. Giachini FR, Galaviz-Hernandez C, Damiano AE, Viana M, Cadavid A, Asturizaga $\mathrm{P}$, et al. Vascular dysfunction in mother and offspring during preeclampsia: contributions from latin-American countries. Current hypertension reports. 2017;19(10):83.

3. Bendich A, Deckelbaum RJ. Preventive nutrition: the comprehensive guide for health professionals: Springer; 2016.

4. Schutte J, Steegers E, Schuitemaker N, Santema J, de Boer K, Pel M, et al. Rise in maternal mortality in the Netherlands. BJOG: An International Journal of Obstetrics \& Gynaecology. 2010;117(4):399-406.

5. Williams D, editor Long-term complications of preeclampsia. Seminars in nephrology; 2011: Elsevier.

6. Meads C, Cnossen J, Meher S, Juarez-Garcia A, Ter Riet G, Duley L, et al. Methods of prediction and prevention of pre-eclampsia: systematic reviews of accuracy and effectiveness literature with economic modelling. 2008.

7. Vatten LJ, Skjærven R. Is pre-eclampsia more than one disease? BJOG: An International Journal of Obstetrics \& Gynaecology. 2004;111(4):298-302.

8. Benton SJ, Leavey K, Grynspan D, Cox BJ, Bainbridge SA. The clinical heterogeneity of preeclampsia is related to both placental gene expression and placental histopathology. American journal of obstetrics and gynecology. 2018;219(6):604. e1-. e25.

9. Salles AM, Galvao TF, Silva MT, Motta LC, Pereira MG. Antioxidants for preventing preeclampsia: a systematic review. ScientificWorldJournal. 2012;2012:243476.

10. Blair SN, Brodney S. Effects of physical inactivity and obesity on morbidity and mortality: current evidence and research issues. Med Sci Sports Exerc. 1999;31(11 Suppl):S646-62.

11. Polyzos NP, Tzioras S, Mauri D, Papanikolaou EG. Combined vitamin C and $\mathrm{E}$ supplementation for preeclampsia: no significant effect but significant heterogeneity? Hypertens Pregnancy. 2012;31(3):375-6.

12. Spinnato JA, 2nd, Freire S, Pinto ESJL, Cunha Rudge MV, Martins-Costa $\mathrm{S}$, Koch MA, et al. Antioxidant therapy to prevent preeclampsia: a randomized controlled trial. Obstet Gynecol. 2007;110(6):1311-8.

13. Sánchez-Aranguren LC, Prada CE, Riaño-Medina CE, Lopez M. Endothelial dysfunction and preeclampsia: role of oxidative stress. Frontiers in physiology. 2014;5:372. 
14. Murphy MS, Tayade C, Smith GN. Evidence of inflammation and predisposition toward metabolic syndrome after pre-eclampsia. Pregnancy Hypertension: An International Journal of Women's Cardiovascular Health. 2015;5(4):354-8.

lial dysfunction and preeclampsia: role of oxidative stress. Frontiers in physiology. 2014;5:372.

14. Murphy MS, Tayade C, Smith GN. Evidence of inflammation and predisposition toward metabolic syndrome after pre-eclampsia. Pregnancy Hypertension: An International Journal of Women's Cardiovascular Health. 2015;5(4):354-358. 


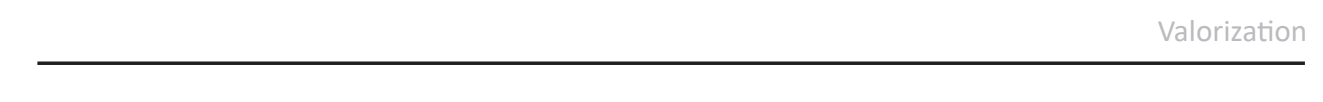

213 


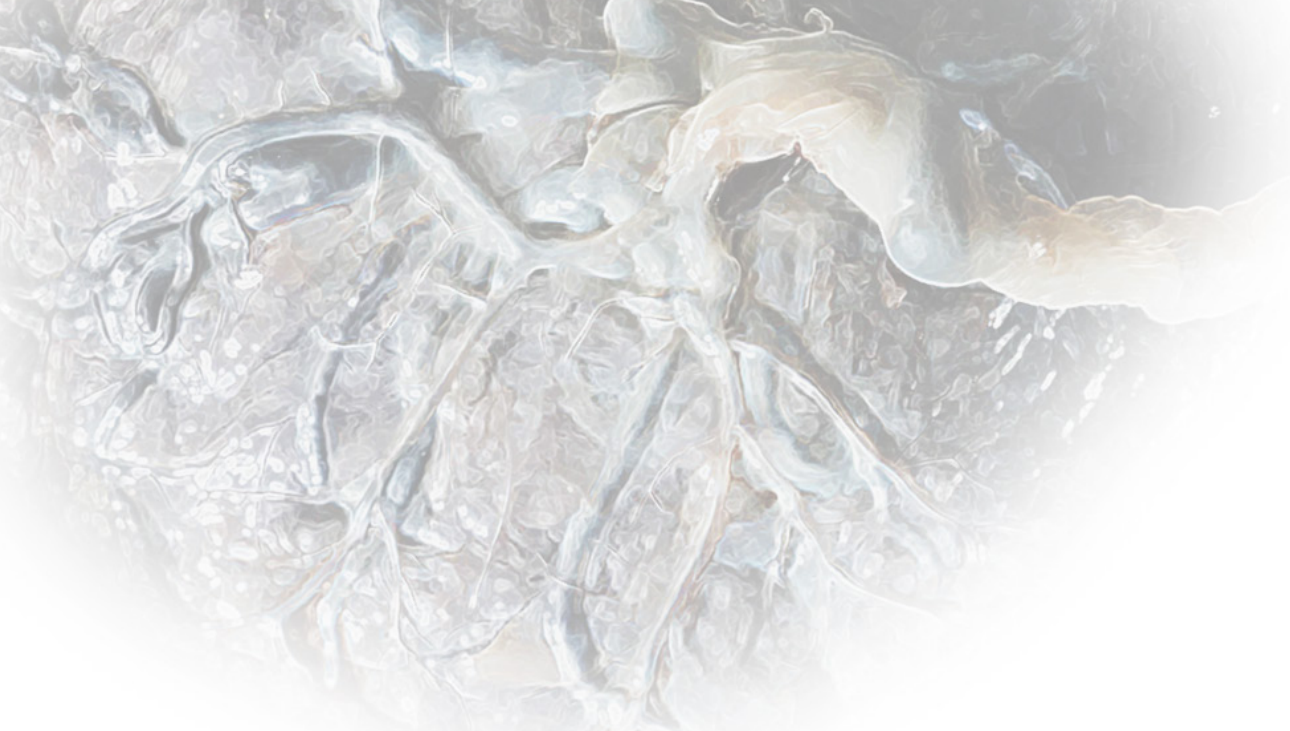




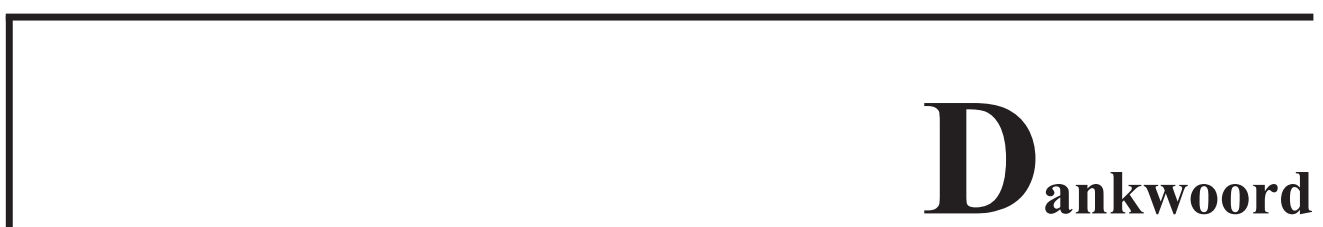


In dit dankwoord wil ik graag een aantal mensen in het bijzonder bedanken voor de steun en hun bijdrage aan deze bijzondere periode van mijn leven. Ook wil ik de NUTRIM GP commissie bedanken, voor het unieke programma dat zij hebben opgezet dat mij en al vele andere de kans heeft geboden om hun eigen project vorm te geven binnen een $\mathrm{PhD}$ project.

Ger, vriend, ik kan niet anders dan met jou te beginnen. Ik kan me onze eerste ontmoetingen nog herinneren als de dag van gisteren. Je kwam samen met Paul langs in het lab tijdens mijn master stage op de Toxicologie afdeling, omdat jullie hadden gehoord dat er een Belgische jonge in het lab bezig was om cellen te isoleren uit een placenta, waar naast cellen ook heel veel bloedvaten in zitten. Ik herinner me je enthousiasme om eens wat bloedvaten te isoleren uit deze placenta en om samen met Paul te kijken of ze geschikt waren voor experimenten uit te voeren met de myograaf (een toestel waar je bloedvaten in kunt monteren en vervolgens hun contractie kan meten). Dan ging het vaak van, kijk Philippe, waaaaaw, hij doet het! 1, nee 2 wooooow $4 \mathrm{~N} / \mathrm{m}$ !, dat is echt een SUPER bloedvat! Geweldig vond ik dat! Van een placenta kwamen er al gauw twee, drie... placenta's en we waren vertrokken! De vele uren dat we samen in het lab bezig zijn geweest tot vaak in de late uurtjes, de vele "koffietjes" tussendoor en onze gesprekken, maakte van deze periode een onvergetelijke tijd!

Daarnaast wil ik ook zeker mijn promotieteam die vol vertrouwen achter mij stond bedanken. Salwan Al-Nasiry, ook ons eerste gesprek kan ik me nog heel goed voor de geest halen. Ik kwam langs voor een sollicitatiegesprek voor mijn junior master stage en al meteen voelde ik dat we een klik hadden. Je vertelde me over de onderzoekslijnen van jullie groep samen met Marc Spaanderman en dat jullie heel interessant "overschot materiaal" hadden, placenta's. Tijdens deze stage, heb ik veel inspirerende gesprekken met je gehad die heel aanstekelijk waren en me heel enthousiast hebben gemaakt. Je gaf me ook de totale vrijheid, om mijn gang te gaan en zelf iets van deze stage te maken. In de daaropvolgende zomervakantie zijn we samen naar Cambridge gereden om daar een zomercursus over placenta's bij te wonen, een fantastische tijd! Salwan, je was een inspiratiebron voor mij en de vrijheid en je vertrouwen in mij hebben me de ideale werkomgeving geboden waar ik alle kansen kon benutten. Paul Schiffers, al gauw tijdens de eerste experimenten die we met de placentale bloedvaten uitvoerde, werd je enthousiast en ontstond er tussen ons en Ger een heel fijne dynamiek. We hebben veel plezier gehad, kwamen soms niet bij van het lachen en ik kon op elke moment bij je terecht als ik een vraag had, of als er iets op mijn lever lag. Ik vond het dan ook geweldig dat ik je kon vragen of je mijn co-promotor wilde worden, toen ik mijn onderzoeksvoorstel voor mijn $\mathrm{PhD}$ 
wilde indienen. Het was een waar genoegen met jou te mogen samenwerken! Aalt Bast, hoewel je kritische blik op mijn Vlaamse woordenschat vaak vanuit een onverwachte hoek binnen kwam sluipen, hebben we veel gezellige gesprekken gehad! Het was altijd fijn te voelen dat je enthousiast was over mijn project. Met je oog voor details en je doortastende vragen was het altijd fijn mijn resultaten aan je voor te leggen. Frederik-Jan van Schooten, ik wil je graag bedanken dat je naast mijn PhD project ook mijn plannen en mijn opleiding tot Toxicoloog hebt gesteund. Tijdens het congres in Baltimore, hebben we elkaar nog beter leren kennen, dat was een geweldige tijd! Ook ben ik via jou in contact gekomen met een aantal zeer interessante mensen, waaronder je vriendin in New York Jia Chen, die ook placentaal onderzoek uitvoert. Ik ben heel blij dat je deel uit maakte van deze periode en hoop in de toekomst nog met je te mogen samenwerken!

Naast de leden van mijn promotieteam, wil ik nog graag 2 mensen in het bijzonder bedanken. Ulrike Rango, tijdens mijn master stage bij Salwan, ben ik ook in contact gekomen met jou. Jij had ook interesse en veel ervaring binnen het placentaal onderzoek. Al snel hadden we samen een onderzoek opgezet, dat we tijdens mijn $\mathrm{PhD}$ periode verder hebben kunnen uiteenzetten. Door jou aangename persoonlijkheid, jouw kritische blik en oog voor details was het een waar genoegen en verrijking om met jou te mogen samen werken! Alex Remels, wat begon als een klein projectje naast mijn bloedvaten werk, groeide al snel uit tot een significant gedeelde van mijn uiteindelijke proefschrift. Door jouw gedrevenheid en enthousiasme in nieuwe onderzoeksvelden, gerelateerd aan mitochondriën, stond je open om samen met mij te duiken in de betrokkenheid van mitochondriën in de ziekte preeclampsie. Ik heb heel veel van je geleerd, door mij de mogelijkheid te geven nieuwe technieken aan te leren en altijd uitgebreide suggesties te leveren op mijn manuscripten. Ook hoop ik dat we onze samenwerking verder kunnen doortrekken in de toekomst.

Christy, doordat jij als PhD student bij Alex gelijkaardige experimenten deed, heb ik heel veel aan jou gehad. Je stond altijd als eerste klaar om even bij te springen en even te helpen als ik een nieuw experiment ging uitvoeren en ik even iets niet wist staan of vergeten was op welk knopje ik nou weer moest drukken ;-) bedankt daarvoor! De koffietjes samen met Ger en onze vele gesprekken waren vaak een aangename uitlaat klep en aanschouw ik als een en al gezelligheid. Ook was onze gemeenschappelijk trip na het congres in Baltimore in Washington D.C. en New York samen met Ger, onvergetelijk! Ik ben ervan overtuigd dat we na mij $\mathrm{PhD}$ periode nog contact zullen blijven houden en regelmatig een keer zullen afspreken. 
Verder zou ik mijn PhD collega's willen bedanken voor de geweldige sfeer. Carmen en Kim voor de oneindige praatjes op jullie kamer en de gezellige etentjes bij jullie thuis. Timme als kamergenoot hebben we veel lief en leed gedeeld. George, Robert, Sven and Stephan, thanks for the nice time, the inspiring talks, the jokes and the funny moments we had together! Shan, thanks for the food we shared, the nice talks and the trip we made together with Wembo, Ying Yi and Wei. Caitlin, Martijn, Wouter, Wessel, Charlotte en Stephanie, bedankt voor de gezelligheid en de leuke koffietjes tussendoor!

Ook zou ik heel graag mijn dank willen uitdrukken aan de studenten (Nadine, Dominique, Stijn, Titus, Annemarie, Iris G. en Iris K., Daan, Yannick en Louise) die bij mij stage hebben gelopen en zo ook een belangrijke bijdrage hebben geleverd aan dit proefschrift. Ik heb ervan genoten jullie te mogen begeleiden, wat altijd zorgde voor een gezellige drukte tijdens mijn werkdag en waar ik ook mooie vriendschappen aan over heb gehouden! Jullie hebben kleur gegeven aan deze periode, bedankt!

Dan zou ik ook heel graag mijn ouders, plus papa's en grootouders willen bedanken voor hun onvoorwaardelijke steun en de kansen die jullie me hebben geboden in mijn leven. Dit hoofdstuk is te kort om uit te drukken wat jullie samen met mijn zuS en schoonfamilie voor me betekenen en voor me hebben gedaan. Heel erg bedankt!

En dan, mijn lieve vrouw $\mathbf{S a}$. Ik ben een ongelofelijke gelukzak dat ik jou altijd naast me heb. Je bent er altijd voor me en bent men beste maatje. Je hebt me onvoorwaardelijk gesteund en was altijd begripvol als ik weer eens laat thuiskwam, omdat ik nog iets moest afmaken, of als een experiment weer was uitgelopen. Je bent een geweldige vrouw en het is altijd fijn thuiskomen met jou. Ook ben ik ongelofelijk trots op jou, omdat je zo een fantastische mama bent voor onze prachtige dochter Philine, die me extra motiveert om voor een mooie toekomst te vechten. 


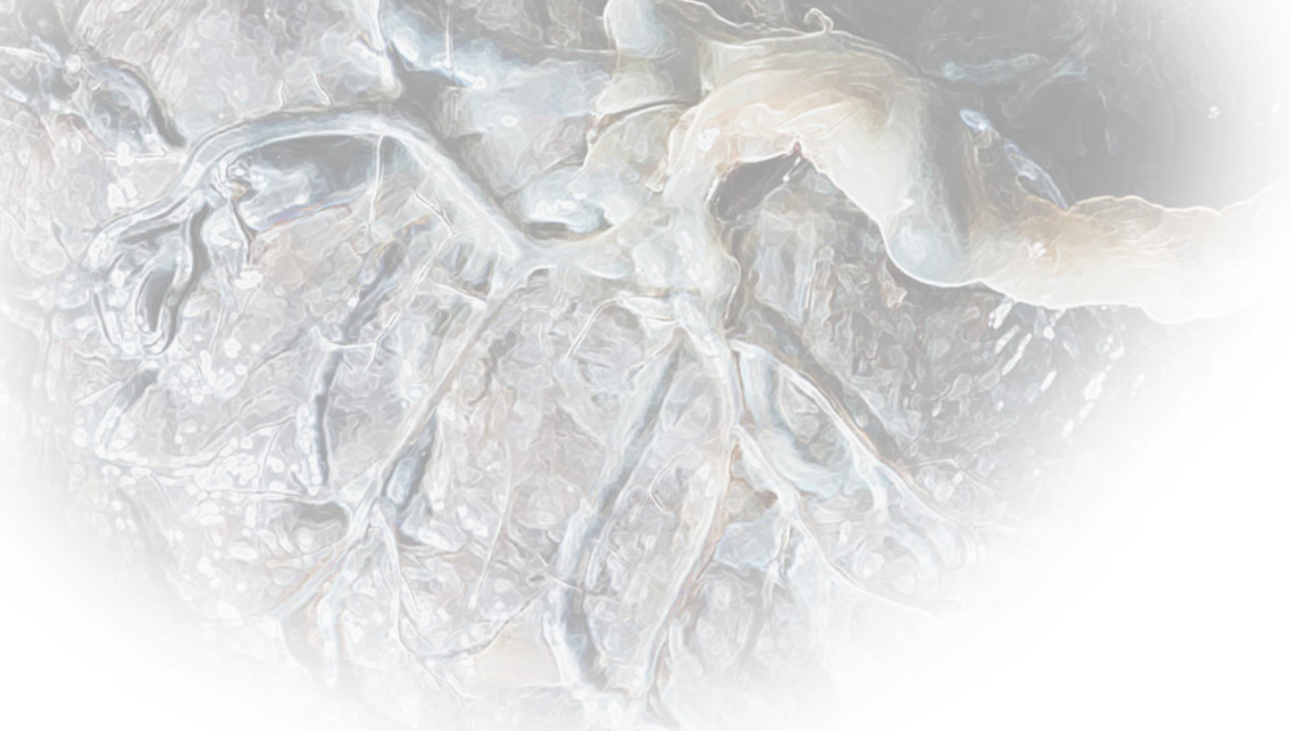




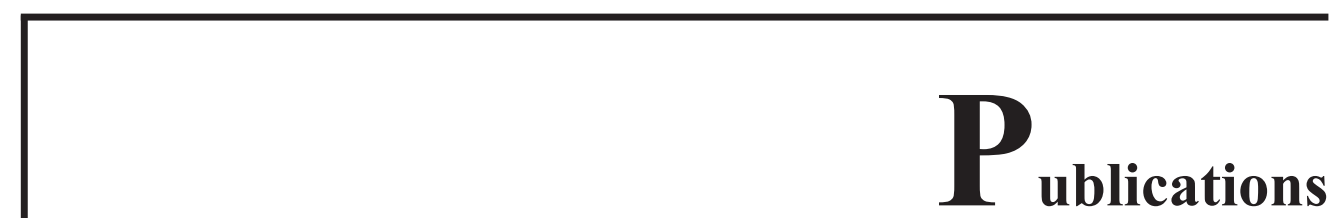


Mireille M.J.P.E. Sthijns, Paul M. Schiffers, Ger M. Janssen, Kristien J.A. Lemmens, Bart Ides, Philippe Vangrieken, Freek G. Bouwman, Edwin C. Mariman, Irina Pader, Elias S.J. Arnér, Katarina Johansson, Aalt Bast, Guido RMM Haenen. "Rutin protects against $\mathrm{H} 2 \mathrm{O} 2$-triggered impaired relaxation of placental arterioles and induces Nrf2-mediated adaptation in human umbilical vein endothelial cells exposed to oxidative stress." Biochimica et Biophysica Acta (BBA)-General Subjects 1861.5 (2017): 1177-1189.

Ming Zhang, Paul Schiffers, Ger Janssen, Misha Vrolijk, Philippe Vangrieken, Guido R.M.M. Haenen. "The cardiovascular side effects of Ma Huang due to its use in isolation in the Western world." European Journal of Integrative Medicine 18 (2018): 18-22.

Philippe Vangrieken, Salwan Al-Nasiry, Ger M.J. Janssen, Antje R. Weseler, Marc E. Spaanderman, Aalt Bast, Paul M.H. Schiffers. "The direct and sustained consequences of severe placental hypoxia on vascular contractility." PloS one 13.8 (2018).

Philippe Vangrieken, Sizzle F. Vanterpool, Frederik J. van Schooten, Salwan A1-Nasiry, Peter Andriessen, Ellen Degreef, Joachim Alfer, Boris W. Kramer, Ulrike von Rango. "Histological villous maturation in placentas of complicated pregnancies." Histology and Histopathology (2020): 18205.

Philippe Vangrieken, Salwan Al-Nasiry, Aalt Bast, Pieter A. Leermakers, Christy B. M. Tulen, Paul M. H. Schiffers, Frederik J. van Schooten, Alex H. V. Remels. Placental mitochondrial abnormalities in preeclampsia. Submitted.

Philippe Vangrieken, Salwan Al-Nasiry, Aalt Bast, Pieter A. Leermakers, Christy B.M. Tulen, Ger. M.J. Janssen, Iris Kaminski, Iris Geomini, Titus Lemmens, Paul M.H. Schiffers, Frederik J. van Schooten, Alex H.V. Remels. Hypoxia-induced mitochondrial abnormalities in cells of the placenta. Submitted.

Philippe Vangrieken, Alex H.V. Remels, Salwan Al-Nasiry, Aalt Bast, Ger. M. J. Janssen, Ulrike von Rango, Annemarie Carree, Daan Vroomans, Yannick C.W. Pinckers, Frederik J. van Schooten, Paul M.H. Schiffers. Placental hypoxia-induced alterations in vascular function, morphology, and endothelial barrier integrity. Hypertension Research, (2020), accepted. 
Khrystyna O. Semen, Ostap H. Yavoskyi, Olha P. Yelisyeyeva, Ger M. Janssen, Philippe Vangrieken, and Aalt Bast. Interaction between food-derived antioxidants and sildenafil: potential therapeutic implications in pulmonary arterial hypertension. In preparation.

Carmen Severens-Rijvers, Peter Nikkels, Philippe Vangrieken, Marc Spaanderman, Carine Peutz-Kootstra and Salwan Al-Nasiry. The microvasculature of the placenta: potential pathophysiological significance in placental syndrome. In preparation. 


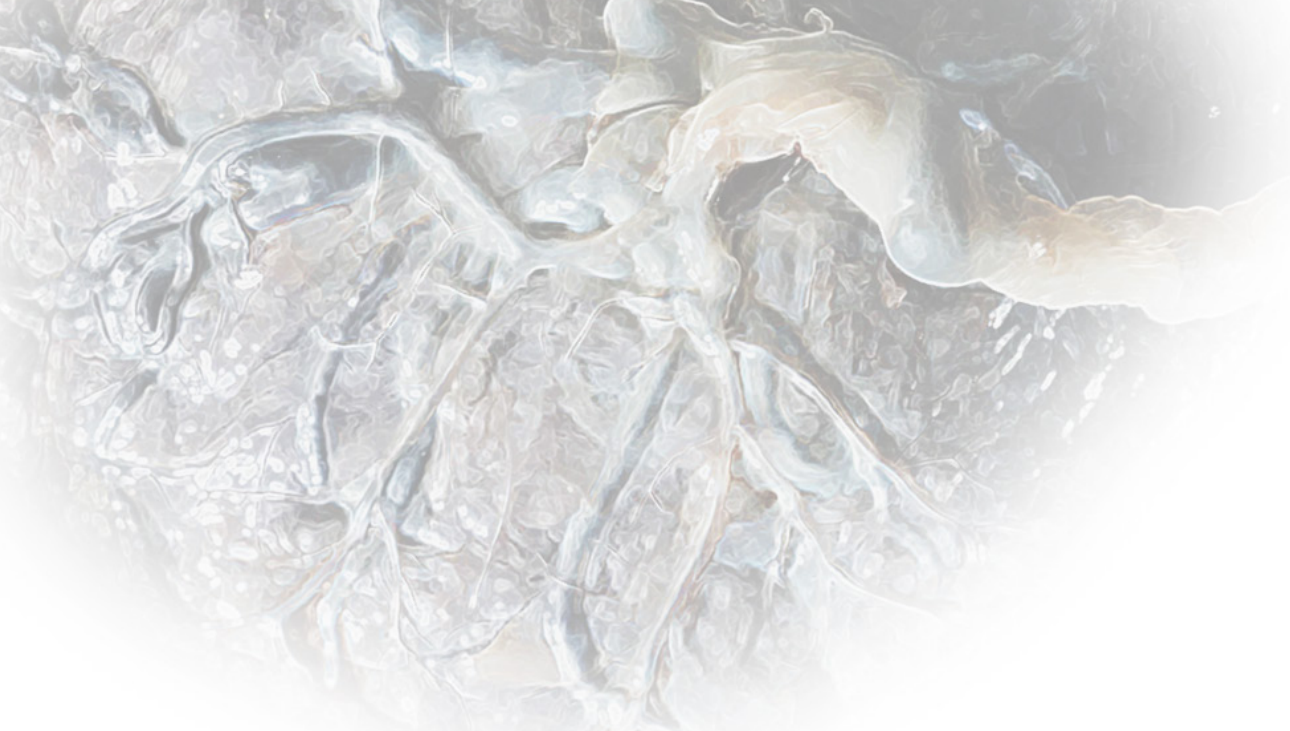




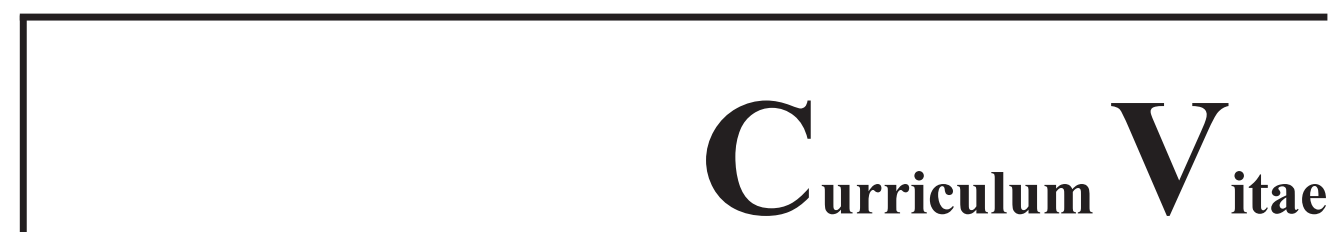



Philippe Vangrieken was born in Lommel, Belgium, on April 29th of 1992. In 2011, he graduated from the secondary school at the Sint-Maria institute in Neerpelt, Belgium. He first started the Bachelor of Sciences program of Biomedical Sciences at the University of Maastricht in 2011. After graduating in 2014, he enrolled in the Master of Science program of Biomedical Sciences of which he graduated in 2016. During his studies, Philippe performed several research projects within the Maastricht University Medical Center+. The first project was performed at the Department of Pharmacology and Toxicology titled "The interaction of flavonoids and collagen fragments: a new mechanism to explain their anti-inflammatory activity". A second research project was performed at the Department of Obstetrics and Gynecology titled "A new standard method to measure placental hyper-maturity", and the last project of his studies was performed at the Department of Pharmacology and Toxicology titled "A vascular in-vitro model for preeclampsia", which was selected for the Dr. Saal van Zwanenberg Master Thesis Price by the Hollandse Maatschappij der Wetenschappen.

During this last research project Philippe submitted a grant proposal, which was awarded with 5000 euro by the Foundation "Gezond Geboren". During this period, Philippe also designed and submitted a grant proposal requesting funding for a $\mathrm{PhD}$ project to the NUTRIM graduate programme, which granted him the funds to perform his PhD research at the Department of Pharmacology and Toxicology of Maastricht University. The execution of the PhD project was performed under the supervision of Prof. dr. Aalt Bast, Prof. dr. Frederik-Jan van Schooten, dr. Salwan Al-Nasiry and dr. Paul M.H. Schiffers. The general aim of this project was to investigate the effects of preeclampsia on placental histological villous maturation and to set up a model for preeclampsia to examine the contribution of mitochondria in the generation of placental oxidative stress, its effect on the placental secretome and its subsequent role in acute and sustained vascular contractility, morphology, and endothelial integrity. During the execution of his $\mathrm{PhD}$ project, Philippe successfully completed the Postgraduate Education in Toxicology training programme and won the CARIM, NUTRIM and Dutch society of Toxicology (NVT) Poster Price. After obtaining his $\mathrm{PhD}$ degree, Philippe will apply for the registration as European Recognized Toxicologist (ERT) and will continue his research at the Department of Internal Medicine at Maastricht University under the supervision of Prof. dr. Casper G. Schalkwijk where he will work on the effect of dietary advanced glycation endproducts on the microcirculation. 